\title{
Compliant Manipulation for Autonomous Search and Rescue Operations
}
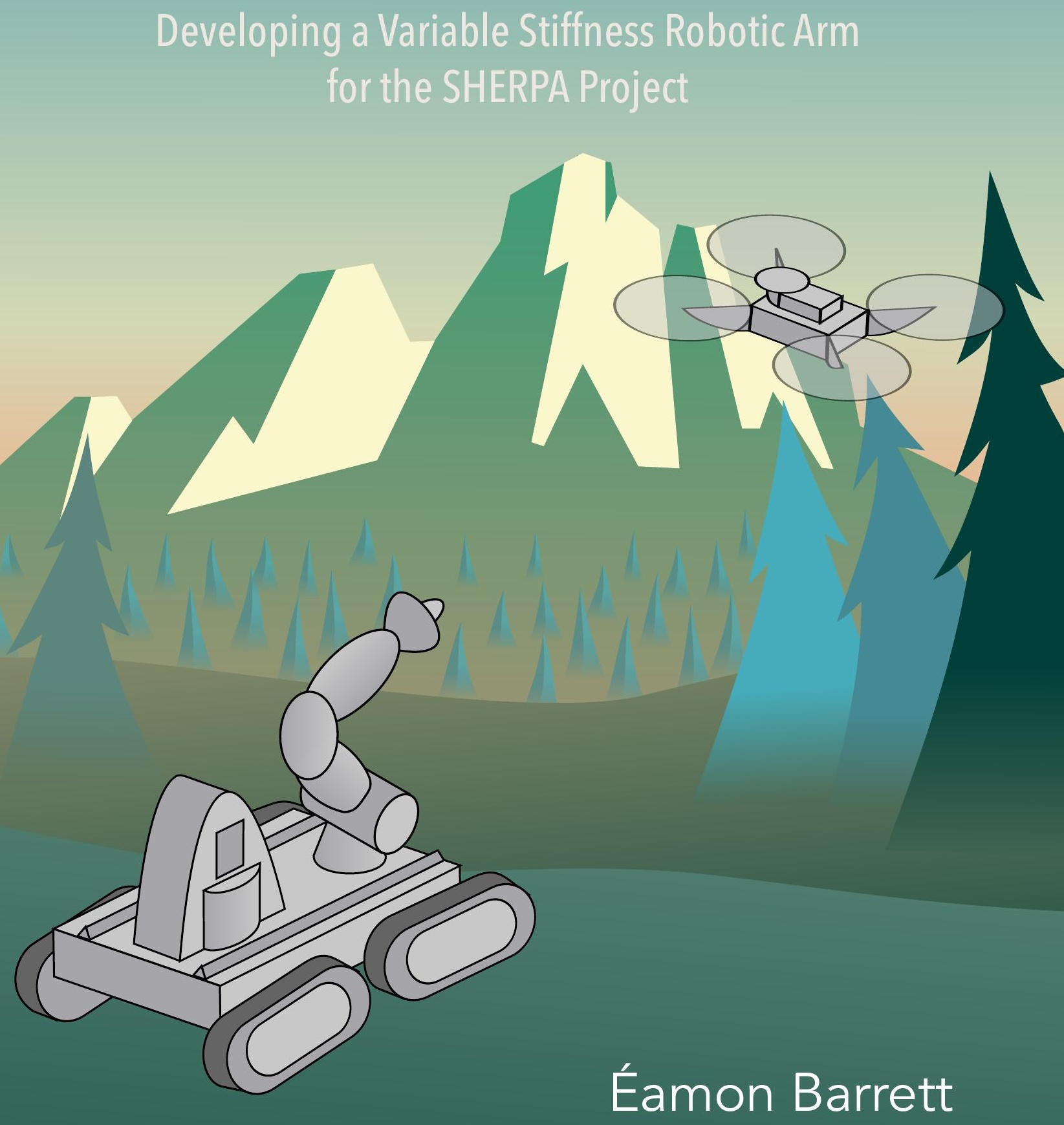


\section{COMPLIANT MANIPULATION FOR AUTONOMOUS SEARCH AND RESCUE OPERATIONS}

DEVELOPING A VARIABLE STIFFNESS ROBOTIC ARM FOR THE SHERPA PROJECT 


\section{Graduation committee}

Chairman and Secretary:

prof.dr.ir. J.N. Kok

University of Twente, the Netherlands

Promotor:

prof.dr.ir. S. Stramigioli

University of Twente, the Netherlands

Members:

prof.dr.ir. C. Melchiorri

University of Bologna, Italy

prof.dr.ir. B. Vanderborght

Vrije Universiteit Brussel, Belgium

prof.dr.ir. V. Evers

prof.dr.ir. D.M. Brouwer

dr.ir. M. Fumagalli

University of Twente, the Netherlands

University of Twente, the Netherlands

Aalborg University Copenhagen, Denmark

The research leading to these results has been carried out at the Robotics and Mechatronics group at the Faculty of Electrical Engineering, Mathematics and Computer Science, University of Twente. The research has received funding from the European Union's Seventh Framework Programme for research, technological development and demonstration as part of the project SHERPA under grant agreement no. 600958.

\section{PMM UNIUESTIY OS SHERPA i}

Publisher:

University of Twente

P.O. Box 217, 7500 AE, Enschede, The Netherlands

ISBN: $\quad 978-90-365-4575-4$

DOI: $\quad 10.3990 / 1.9789036545754$

Copyright $@$ 2018, by Éamon Barrett, Enschede, The Netherlands.

Printed by Ipskamp Printing.

Cover design by Éamon Barrett. 


\section{COMPLIANT MANIPULATION FOR AUTONOMOUS SEARCH AND RESCUE OPERATIONS}

\section{DEVELOPING A VARIABLE STIFFNESS ROBOTIC ARM FOR THE SHERPA PROJECT}

\section{DISSERTATION}

to obtain

the degree of doctor at the University of Twente, on the authority of the rector magnificus, prof. dr. T.T.M. Palstra, on account of the decision of the graduation committee, to be publicly defended on Friday $22^{\text {nd }}$ of June 2018 at 16.45

by

\section{Éamon Barrett}

born on 10 Febuary 1987

in Hamburg, Germany. 
This dissertation has been approved by:

prof.dr.ir. S. Stramigioli, Promotor

Copyright $\odot$ 2018, by Éamon Barrett, Enschede, The Netherlands. 


\section{SUMMARY}

Autonomous robotic systems are performing an ever-increasing variety of tasks that can not only make our lives simpler, but sometimes even help save them. Disaster response, and search and rescue missions are such an application, where robots can greatly support human rescuers by expanding their capabilities and relieving them of dangerous or routine tasks. This is the goal of the SHERPA project, in which a mixed ground and aerial robotic team with a high degree of autonomy helps to locate missing or injured people in a hostile alpine environment. A compliant manipulator herein services small-scale UAVs, a collaborative task that involves dexterous manipulation in an unfamiliar environment, and potentially impacts or collisions. For this reason it is equipped with Variable Stiffness Actuators VSAs, which allow it to tune its mechanical end effector stiffness, and to interact with the environment in a passively compliant way. This thesis presents the design and control of this novel manipulator and its components, its integration with the other agents of the SHERPA team, and experimental validation of the mission.

A core component of this compliantly actuated system are a number of VSAs, which allow safe and dexterous interaction with the environment. The analysis of their design focuses on modeling the internal energy flows and optimization of their mechanical energy storage elements. The arms actuation topology and its effect on the achievable workspace compliance, are investigated, and a thorough mathematical framework for solving associated control problems introduced. The mechatronic design of the robotic arm and its components is presented, including its kinematics, the design of several differentially coupled joints, and a custom gripper, developed to latch into an interface mounted on the UAV to ensure robust grasping under misalignment. The arm has been successfully integrated with the rest of the SHERPA team through a control and delegation framework which allows the agents to autonomously plan and execute complex missions. The completion of the arms main task of replacing a landed UAVs battery is used to demonstrate the systems capabilities, and underlines the role such automated systems can play in supporting and improving search and rescue operations.

A number of research questions relevant to the fields of compliant manipulation, 
collaborative robotics, and the coordination and control of robotically aided search and rescue operations have been addressed in the course of this research, resulting in a novel robotic manipulator that demonstrated its ability to perform complex collaborative operations. 


\section{SAMENVATTING}

Autonome robotische systemen voeren een grooiende aantal van diverse taken uit die ons leven niet alleen eenvoudiger kunnen maken, maar soms zelfs kunnen helpen om ze te redden. Rampenrespons en opsporings- en reddingsoperaties zijn een zulke toepassing, waarbij robots menselijke hulpverleners in hoge mate kunnen ondersteunen door hun mogelijkheden uit te breiden en hen te ontlasten van gevaarlijke of routinetaken. Dit is het doel van het SHERPA project, waarbij een gemengd gronden luchtrobotteam met een hoge mate van autonomie helpt om vermiste of gewonde mensen te lokaliseren in een alpiene omgeving. Een compliante manipulator ondersteunt hierin kleinschalige UAV's, een collaboratieve taak die behendige manipulatie in een onbekende omgeving eist, met mogelijke impacts of botsingen. Daarom is hij uitgerust met Variable Stijfheid Actuatoren, die het mogelijk maken om zijn mechanische eindeffector stijfheid af te stellen, en om te interageren met de omgeving op een passief compliante manier. Dit proefschrift presenteert het ontwerp en de regeling van deze nieuwe manipulator en zijn componenten, zijn integratie met de overige actoren van het SHERPA team, en experimentele validatie van de missie.

Kerncomponenten van dit systeem zijn een aantal VSA's die een veilige en soepele interactie met de omgeving mogelijk maken. De analyse van hun ontwerp richt zich op het modelleren van de interne energiestromen en het optimaliseren van hun mechanische energieopslagelementen. De actuatortopologie van de arm en het effect ervan op de haalbare werkplekcompliance worden onderzocht en er wordt een grondig wiskundig kader geïntroduceerd voor het oplossen van bijbehorende regelproblemen. Het mechatronische ontwerp van de robotarm en zijn componenten wordt gepresenteerd, met inbegrip van de kinematica, het ontwerp van verschillende differentieel gekoppelde verbindingen, en een op maat gemaakte grijper, ontwikkeld om in een interface vast te klikken dat op de UAV gemonteerd is, om een robuuste grip, zelvs onder verkeerde uitlijning te garanderen. De arm is succesvol geïntegreerd met de rest van het SHERPA-team door middel van een regelings- en delegatieraamwerk dat de actoren in staat stelt om zelfstandig complexe missies te plannen en uit te voeren. De voltooiing van de belangrijkste taak van de arm, het vervangen van de batterij van een gelande UAV, wordt gebruikt om de mogelijkheden van het systeem te demonstreren 
en onderstreept de rol die dergelijke geautomatiseerde systemen kunnen spelen bij het ondersteunen en verbeteren van opsporings- en reddingsoperaties.

Een aantal onderzoeksvragen die relevant zijn op het gebied van compliante manipulatie, collaboratieve robotica, en de coördinatie en regeling van robotisch ondersteunde opsporings- en reddingsoperaties zijn in de loop van dit onderzoek geadresseerd, wat heeft geresulteerd in een nieuwe robotmanipulator die heeft aangetoond in staat te zijn complexe collaboratieve operaties uit te voeren. 


\section{ZUSAMMENFASSUNG}

Autonome robotische Systeme erfüllen immer vielfältigere Aufgaben, die unser Leben nicht nur vereinfachen, sondern manchmal sogar retten können. Katastrophenschutz und Such- und Rettungseinsätze sind solche Anwendungen, bei der Roboter menschliche Retter in hohem Maße unterstützen können, indem sie ihre Einsatztfähigkeiten erweitern und sie von gefährlichen oder repetitiven Aufgaben befreien. Dies ist das Ziel des SHERPA-Projekts, bei dem ein gemischtes Team aus fliegenden und bodengebundenen Robotern mit einem hohen Maß an Autonomie hilft, vermisste oder verletzte Menschen in einer wiedrigen alpinen Umgebung zu lokalisieren. Ein komplianter Manipulator unterstüzt hierbei kleine UAVs, eine kollaborative Aufgabe, die geschickte Manipulation in einer unbekannten Umgebung und potenzielle Kollisionen beinhaltet. Aus diesem Grund ist er mit VSAs, Aktuatoren mit variabler Steifheit, ausgestattet, die es ihm ermöglichen, die mechanische Steifigkeit seines Endeffektors einzustellen und in passiver Weise mit der Umgebung zu interagieren. Diese Arbeit stellt das Design und die Regelung dieses neuartigen Manipulators und seiner Komponenten, seine Integration mit den übrigen Aktoren des SHERPA-Teams und die experimentelle Validierung der Mission vor.

Eine Kernkomponente dieses nachgiebig betriebenen Systems sind eine Reihe von VSAs, die eine sichere und geschickte Interaktion mit der Umwelt ermöglichen. Die Analyse ihres Designs konzentriert sich auf die Modellierung der internen Energieströme und die Optimierung ihrer mechanischen Energiespeicherelemente. Die Aktuator-Topologie des Arms und ihr Einfluss auf die erreichbare Komplianz des Endeffektors werden untersucht und ein fundierter mathematischer Rahmen zur Lösung der damit verbundenen Regelungsprobleme vorgestellt. Das mechatronische Design des Roboterarms und seiner Komponenten wird präsentiert, einschlielich seiner Kinematik, der Konstruktion mehrerer unterschiedlich gekoppelter Gelenke und eines speziell angefertigten Greifers, der so entwickelt wurde, dass er in eine an dem UAV angebrachte Schnittstelle einrastet, um ein robustes Greifen selbst unter fehlerhafter Ausrichtung zu gewährleisten. Der Arm wurde erfolgreich mit dem Rest des SHERPATeams durch ein Regelungs- und Delegations-Rahmenwerk integriert, das es den Aktoren ermöglicht, komplexe Missionen autonom zu planen und durchzuführen. Die 
Erfüllung der Hauptaufgabe des Arms, die Batterie eines gelandeten UAVs zu ersetzen, dient der Demonstration der Fähigkeiten des Systems und unterstreicht die Rolle, die solche automatisierten Systeme bei der Unterstützung und Verbesserung von Suchund Rettungsaktionen spielen knnen.

Eine Reihe von Forschungsfragen, die für kompliante Manipulation, kollaborative Robotik und die Koordination und Steuerung von robotergestützten Such- und Rettungsoperationen relevant sind, wurden im Rahmen dieser Forschungsarbeit behandelt, was zur Entwicklung eines neuartigen robotischen Manipulators führte, der seine Eignung zur Durchführung komplexer kollaborativer Operationen unter Beweis stellten konnte. 


\section{CONTENTS}

Summary

Samenvatting iii

Zusammenfassung $\quad$ v

1 Introduction 1

1.1 The SHERPA Project . . . . . . . . . . . . . . . . 2

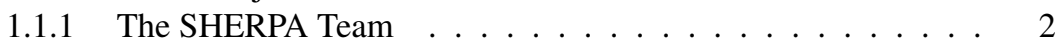

1.1.2 Winter Scenario . . . . . . . . . . . . . . . 5

1.1.3 Summer Scenario . . . . . . . . . . . . . . . . . 6

1.1 .4 Mission Outline . . . . . . . . . . . . . . . . 7

1.1.5 Requirements . . . . . . . . . . . . . . . . 8

1.2 Robotic Manipulation . . . . . . . . . . . . . . . . . . 9

1.2.1 Compliant Actuation . . . . . . . . . . . . . . . 9

1.2.2 Collaborative Robotics . . . . . . . . . . . . . . . . . 10

1.3 Research Objective . . . . . . . . . . . . . . . . . . . 12

1.4 Contribution . . . . . . . . . . . . . . . . . . 14

1.5 Thesis Outline . . . . . . . . . . . . . . . . 15

2 Energy Storage and Spring Design 17

2.1 Introduction . . . . . . . . . . . . . . . . . . . 18

2.2 Classification and Modeling of VSAs . . . . . . . . . . . . . . . 19

2.2.1 Operating Principles . . . . . . . . . . . . . . . . . . . . 19

2.2.2 Port-based Model . . . . . . . . . . . . . . . . 20

2.2 .3 VSA Designs . . . . . . . . . . . . . . . . 23

2.3 Design Evaluation . . . . . . . . . . . . . . . . . 26

2.3.1 Energy Efficient Actuation . . . . . . . . . . . . . . . 27

2.3.2 Energy Storage Capacity . . . . . . . . . . . . . . . . . . 28

2.4 Design for a Lever Arm Based VSA . . . . . . . . . . . . . . 30 
2.4.1 Kinematic Model . . . . . . . . . . . . . . . . . 30

2.4.2 Torque-Deflection Workspace ............ 32

2.4 .3 Internal Loads . . . . . . . . . . . . . . . . . . . . . . . . . . . . . . 33

2.4 .4 Design Guidelines . . . . . . . . . . . . . . . . 33

2.5 Design Methodology for Elastic Elements . . . . . . . . . . . . . . . . . . . 34

2.5.1 Elastic Energy Storage . . . . . . . . . . . . . . 35

2.5.2 Mounting Volume and Spring Type . . . . . . . . . . . . 37

2.5.3 Euler-Bernoulli Beam ...................... 38

2.5.4 Optimizing Spring Parameters . . . . . . . . . . . . . . . 39

2.5.5 Spring Materials . . . . . . . . . . . . . . . . 41

2.5.6 Finite Element Analysis . . . . . . . . . . . . . 43

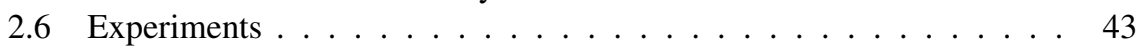

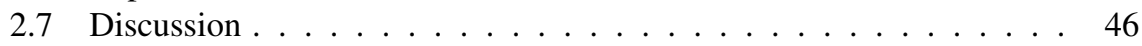

2.8 Conclusions . . . . . . . . . . . . . . . . . 46

3 Mechatronic Design of the Arm System 49

3.1 Introduction . . . . . . . . . . . . . . . . 50

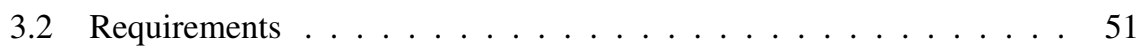

3.2 .1 The SHERPA Mission . . . . . . . . . . . . . . 51

3.2 .2 Design Requirements . . . . . . . . . . . . . . . 52

3.3 Kinematic Analysis . . . . . . . . . . . . . . . . 52

3.3.1 Kinematic Structure . . . . . . . . . . . . . 53

3.3.2 Workspace Analysis . . . . . . . . . . . . . . 54

3.3.3 Workspace Compliance .............. 56

3.4 Mechanical Design . . . . . . . . . . . . . . . . . 57

3.4.1 Actuator Requirements . . . . . . . . . . . . . . . . . 57

3.4 .2 Shoulder Joint . . . . . . . . . . . . . . . . . . . . . . . . . . . . . . . . . 59

3.4 .3 Elbow Joint . . . . . . . . . . . . . . . 61

3.4 Wrist Joint . . . . . . . . . . . . . . . 61

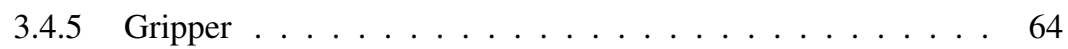

3.4 .6 System Identification . . . . . . . . . . . . . . . . . . . . . . . . . 64

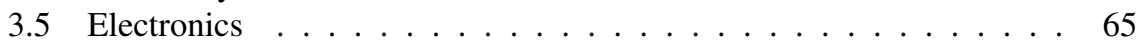

3.6 Software Architecture . . . . . . . . . . . . . . . . 66

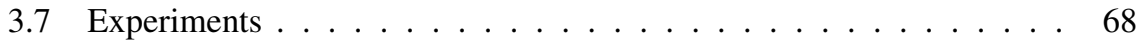

3.7.1 Workspace Compliance .................. 68

3.7 .2 Battery Replacement . . . . . . . . . . . . . . . 70

3.8 Conclusions . . . . . . . . . . . . . . . . . . 70

4 The SHERPA Gripper $\quad 73$

4.1 Introduction . . . . . . . . . . . . . . . . . . . . . 74

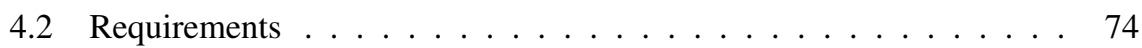

4.3 Engagement Mechanism . . . . . . . . . . . . . . . . . . 75

4.4 Latching Mechanism . . . . . . . . . . . . . . . . . 76 
4.4.1 Working Principle . . . . . . . . . . . . . . 76

4.4.2 Kinematic Analysis . . . . . . . . . . . . . . 77

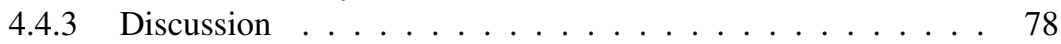

4.5 Actuation Mechanism . . . . . . . . . . . . . . . . . . 78

4.5.1 Linkage Mechanism . . . . . . . . . . . . . . . . 78

4.5.2 Cam Mechanism . . . . . . . . . . . . . . . . . . 80

4.5 .3 Discussion ................... 81

4.6 Mechatronic Implementation . . . . . . . . . . . . . . . . . . . 81

4.6.1 Fingers \& Linear Guide . . . . . . . . . . . . . . . . . . . . 82

4.6.2 Cam Mechanism \& Actuation ............. . . . 82

4.6.3 Electronics \& Control . . . . . . . . . . . . . . 83

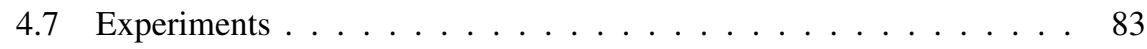

4.8 Conclusions ........................... 84

5 Towards Elastic Control of Semi-Compliant Mechanisms 87

5.1 Introduction . . . . . . . . . . . . . . . . . 88

5.2 Mathematical Foundations . . . . . . . . . . . . . . . 89

5.2.1 Configuration Space of Compliant Mechanisms . . . . . . . . 89

5.2.2 Manipulator Workspace . . . . . . . . . . . . . 90

5.2.3 Manipulator Kinematics . . . . . . . . . . . . . . . . 92

5.3 The Basic Compliant Joint . . . . . . . . . . . . . . . . . . . . . . . . . 94

5.3.1 The Compliant Kinematic Pair . . . . . . . . . . . . . . . 95

5.3.2 Dual Complements and Workspace Decomposition . . . . . . 95

5.4 The Semi-Compliant Serial Mechanism . . . . . . . . . . . . . . 97

5.4.1 Manipulator Kinematics and Total Compliance . . . . . . . . 98

5.4.2 Workspace Decomposition of Serial Mechanisms . . . . . . . 99

5.4.3 Compliance Transformation . . . . . . . . . . . . . . 100

5.4 .4 Projection Operations . . . . . . . . . . . . . . . . . . . 102

5.5 Compliance Metric . . . . . . . . . . . . . . . . . . 102

5.6 Experiments . . . . . . . . . . . . . . . . . 103

5.6.1 Compliance Control . . . . . . . . . . . . . . . . 104

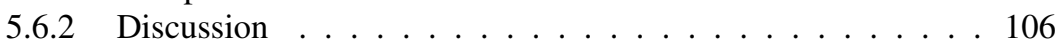

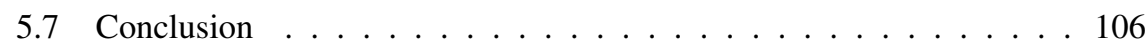

6 Autonomous Battery Exchange 109

6.1 Introduction . . . . . . . . . . . . . . . . . . . 110

6.2 Collaborative Mission Framework . . . . . . . . . . . . . . . . 112

6.3 The SHERPA System . . . . . . . . . . . . . . . . . . . . . 112

6.3.1 Small-Scale UAVs - The SHERPA Wasps . . . . . . . . . . . 113

6.3.2 Service Station - The SHERPA Box . . . . . . . . . . . . . 114

6.3.3 Mobile Base - The Ground Rover . . . . . . . . . . . . . 116

6.3.4 UAV Retrieval - The Robotic Arm . . . . . . . . . . . . . 117

6.4 Experiments .......................... 118 
6.4.1 Battery Exchange Operation . . . . . . . . . . . . 118

6.4 Experimental Results . . . . . . . . . . . . . . 119

6.5 Conclusions . . . . . . . . . . . . . . . . . . 120

7 Conclusions $\quad \mathbf{1 2 5}$

7.1 Findings and Conclusions . . . . . . . . . . . . . . . . 126

7.1.1 Compliant Actuators - VSA and Spring Design . . . . . . . . 126

7.1.2 Compliant Manipulators - Design and Control . . . . . . . . . 127

7.1.3 The SHERPA Project - Collaborative Mission . . . . . . . . . 128

7.2 Future Work . . . . . . . . . . . . . . . . 128

$\begin{array}{lr}\text { Acknowledgements } & 149\end{array}$

$\begin{array}{ll}\text { About the Author } & 150\end{array}$ 


\section{CHAPTER 1}

\section{INTRODUCTION}

This thesis describes the work carried out to develop a compliant manipulator for enabling autonomous search and rescue operations in the context of the European SHERPA project. The manipulator is mounted on a mobile robot, which is part of a robotic team supporting mountain rescue missions. This introductory chapter provides background information and motivation for the project and rescue activities in Alpine environments, including an introduction to the team's agents, the rescue scenario, mission outline, and requirements. A brief outline of compliant and variably compliant manipulation is presented. The chapter further describes the objectives, contributions, and outline of the thesis.

Robotic systems are supporting an ever-increasing variety of tasks that can not only make our lives simpler, but sometimes even help to save them. The applications range from service robotics [1] and inspection or surveillance tasks [2], to disaster response and search and rescue missions $[3,4,5]$, where a robotic system can greatly support humans by expanding their capabilities and relieving them of routine or dangerous tasks. Robots can provide, among other, logistical support and carry out assignments or enter environments that are too dangerous for humans. They also offer the possibility of improving, even revolutionizing, existing or novel applications and operations [6].

As more and more people are drawn to the mountains for recreation, be it for skiing in the winter, or hiking in the summer, search and rescue operations for missing or injured persons require a massive effort in terms of both material and personnel. In Europe alone, national rescue organizations conduct tens of thousands of missions yearly, many requiring expensive equipment like helicopters, and a huge amount of working hours $[7,8,9]$. Incorporating robots in these operations can reduce the strain 
and risk for victims and rescuers, and make rescue quicker, cheaper, and safer.

Robots need the physical and also cognitive and control capabilities to realize this potential. The platforms and embedded systems have to reach a level of maturity at which they can autonomously perform their task, without burdening human rescuers. In order to also physically interact with their surroundings, especially unknown and unstructured environments including humans, robots need to become safe, dexterous, and efficient. Which which is a trend exemplified by the current advancements in collaborative robotics $[10,11]$.

\subsection{The SHERPA Project}

The SHERPA project investigates the Smart collaboration between Humans and ground-aErial Robots for imProving rescuing activities in Alpine environments [12]. The goal of the project is to develop a mixed ground and aerial platform of robots with heterogeneous designs and capabilities, to support search and rescue activities in a real-world hostile environment. SHERPA specifically targets alpine rescue missions. The high requirements associated with these missions, however, ensure that the results are applicable to wider search and rescue or surveillance scenarios.

The project consortium consists of ten partners; seven universities, two SMEs (Small and Medium Companies), and the CAI (Club Alpino Italiano) as end user. Together they are responsible for the mechanical design and construction of the different robotic platforms, tailored to their specialized tasks, including the compliant manipulator presented in this thesis.

The project furthermore addresses a number of research topics about cognition and control that integrate the autonomous robotic platform in human-lead rescue missions. Natural and intuitive interaction methods between robots and humans are required, alongside scene reconstruction, navigation, and cognitive abilities that support situational awareness and decision making. A dynamic cognitive map is used to integrate information from the different agents, which allows the team to react to the unstructured and dynamically changing environments and mission objectives. Each agent participates in a distributed delegation process that plans and allocates tasks according to available resources and mission goals and facilitates hierarchical and heterarchial planning and cooperation. This guarantees flexibility and robustness, and allows different levels of autonomy. The platform is able to operate by itself even in complex situations, but the human can take control at any time, while the platform provides relevant information pertaining to the mission, such as detailed maps or visual data.

\subsubsection{The SHERPA Team}

Figure 1.1 shows a sketch of the SHERPA team on a search and rescue mission in the mountains. It is lead by a human rescuer as the busy genius, who is being followed by the donkey, a ground rover carrying equipment and a service station for the wasps. 


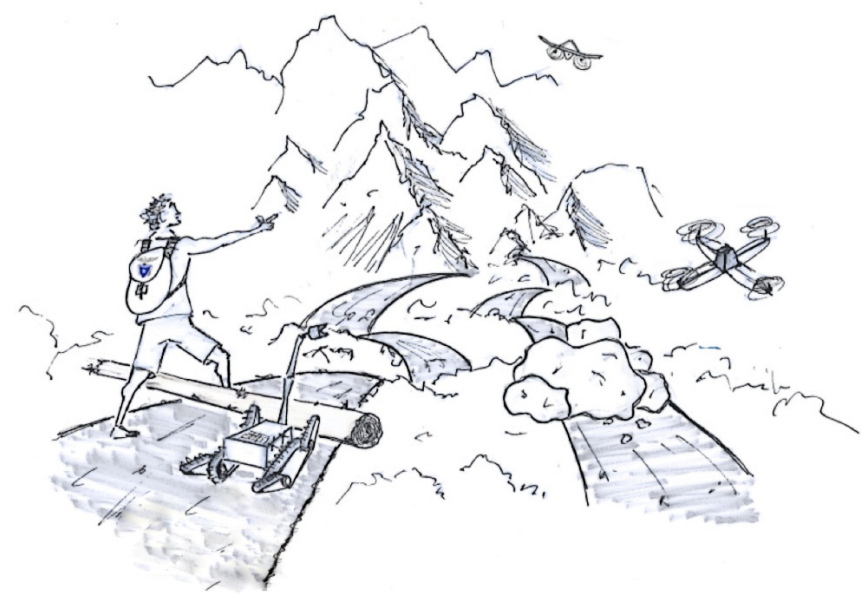

Figure 1.1: SHERPA team, composed of the human rescuer as busy genius, large high flying UAVs as hawks, small-scale UAVs as wasps, and the ground rover as donkey, carrying the robotic arm and service station for the wasps.

These small-scale UAVs (Unmanned Aerial Vehicle) (wasps) serve as eye-in-the-sky for the busy genius, while large-scale UAVs, the hawks, fly at a higher altitude and survey the wider area.

\section{Busy genius}

The human rescuer is the busy genius of the team. As a member of a civil protection organization or rescue service, his knowledge and experience is unmatched, as well as his understanding of the situation and ability to direct the robotic agents accordingly.

Mountain rescue, however, is very demanding and physically exhausting, and the busy genius cannot continuously supervise all the other agents' actions over the course of hour-long missions. Instead he provides sporadic but valuable input to the team, while the platform operates autonomously to support him with essential information. The busy genius can take more complete control of the robotic agents, commanding them to perform certain tasks through a natural and intuitive interface, such as voice commands and gestures [13]. He is able to lead the SHERPA team without specialized training while still actively participating in the rescue task, unlike the dedicated pilots for UAVs that are already being used by some rescue organizations $[14,15]$. Because of the demanding nature of the mission, and the varying level of attention, the busy genius can spare for coordinating the team, the communication and control needs to be very intuitive, and the robot agents take the physical and emotional state of the busy genius into account when requesting or receiving information. 


\section{Wasps}

The SHERPA wasps are small-scale quadrotor UAVs, equipped with small cameras and other sensors for gathering visual information and emergency signals. Their unique point of view and maneuverability make them a highly desired asset to the team, and gives them the ability to effectively survey the vicinity of the busy genius, even if the terrain is inaccessible by foot.

Even though they can operate autonomously, their small size also entails limited range and payload capabilities, so that they need to stay close to their service station. The wasps have been custom built within the project by Aslatech [16], and designed with a quick battery exchange mechanism.

\section{Donkey}

A tracked ground rover serves as the team's donkey, carrying specialized equipment like computational or communication hardware, rescue material, and the service station of the wasps into the operational environment, that otherwise would need to be brought there by the human rescuers. It is characterized by a high degree of autonomy and long endurance, and follows the busy genius along mountain paths. It was custom built within the project by BlueBotics [17] with passive adaptation mechanisms for good off-road capabilities and sufficient power autonomy to support hour-long missions.

The donkey's most important role within the SHERPA scenario is to provide the wasps with a mobile service station, so that their batteries can be exchanged autonomously without burdening the busy genius with cumbersome maintenance tasks. In order to deploy and retrieve the wasps, the donkey is equipped with a variable stiffness robotic manipulator, which enhances its safety, robustness, and adaptability, and which design and control is described in detail in this thesis.

\section{Hawks}

The SHERPA team is completed by the hawks, large-scale UAVs that fly at high altitudes and survey the whole operational area. They complement the wasps and coordinate local activities, construct detailed 3D maps, and can serve as communications hub between the platforms.

Two platforms with complementary properties have been used as the hawks, the senseSoar [18] fixed-wing solar airplane with very long endurance, and the exceptionally robust and reliable Yamaha RMAX [19] unmanned helicopter with large payload and ability to fly in critical weather conditions, which has been augmented for autonomous flight. 


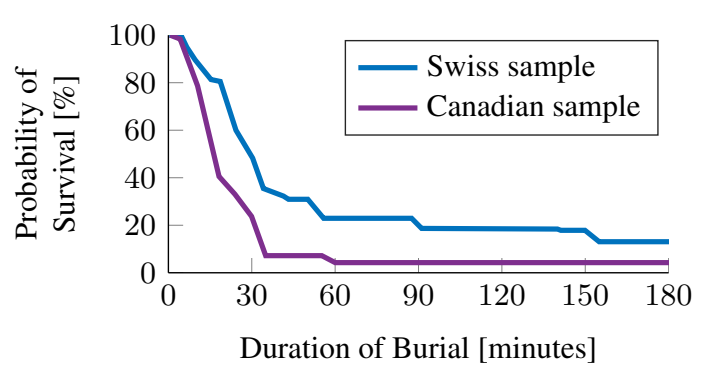

(a)

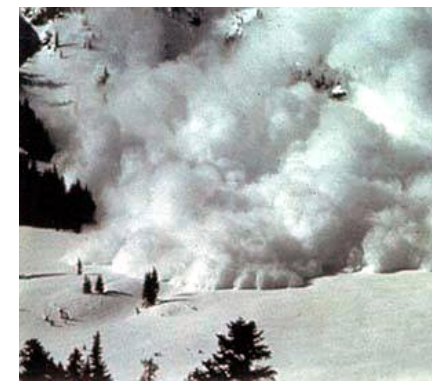

(b)

Figure 1.2: Survival curves for people completely buried in avalanches in Canada and Switzerland by duration of burial (Figure 1.2a, adapted from [20]), and the scene of an avalanche (Figure 1.2b).

\subsubsection{Winter Scenario}

Snow avalanches are a powerful natural phenomenon, and immediately come to mind when thinking about emergency situations in mountain regions. They can pose a significant threat to life, especially during recreational winter sport activities in uncontrolled avalanche terrain, and are the main motivation for the winter scenario considered for the SHERPA project.

While prevention is the best method to avoid harm, rescue devices such as airbags and radio beacons greatly reduce the chance of dying in an avalanche [21], where time is a critical factor. More than $90 \%$ of people rescued within the first 15 to 20 minutes after being buried survive, however the chances for survival rapidly decline $[22,23,24]$. Figure 1.2a shows typical survival curves for people completely buried by avalanches as a function of the duration of burial [20].

Companion rescue plays the most important role for avalanche victims. These first responders are usually also the ones to alarm the rescue services, and may even be able to recover the victims before they arrive. The organized search and rescue then takes place under very adverse conditions. The rescuers and equipment, including probes, receivers, or rescue dogs, need to arrive at the scene as quickly as possible, and search the avalanche. This is made very difficult by the surface of the avalanche, which is usually blocky and difficult to walk on. The use of small UAVs in this stage of the rescue mission is very promising, and first steps have been made towards their application $[25,26]$.

Most people who were fully buried in an avalanche were found using avalanche beacons [24], as shown in Figure 1.3b, such as active avalanche transceivers or passive RECCO transponders, which can significantly reduce the time buried. The primary search with an avalanche receiver consists of finding the signal of the victim's transceiver by marching across the avalanche in a search formation, before it can be localized and extracted. UAVs equipped with avalanche receivers have recently been proposed [27, 28], and a wasp equipped with an avalanche receiver, shown in Figure 


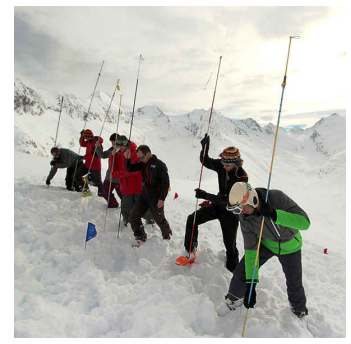

(a)

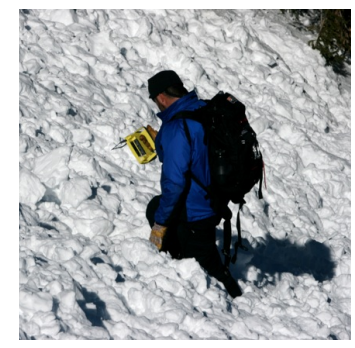

(b)

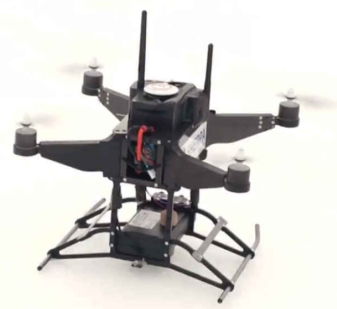

(c)

Figure 1.3: Search for buried avalanche victims with a probe line (Figure 1.3a, image courtesy of Bergrettung Tirol), and with an avalanche transceiver (Figure 1.3b). A SHERPA wasp is equipped with a digital avalanche receiver for finding people buried under an avalanche (Figure 1.3c). The receiver is separated from the body of the UAV to avoid electromagnetic interference.

$1.3 \mathrm{c}$, was explicitly requested by CAI, which benefits even more from the intuitive and largely autonomous control envisaged for the SHERPA wasp.

Because the rescuers need to respond very quickly, and have to search only the difficult and spatially confined area of the avalanche, there is no clear need for the rest of the SHERPA team in the avalanche scenario, which is designed for supporting long endurance missions in large areas.

\subsubsection{Summer Scenario}

Even though the SHERPA system cannot be applied to the avalanche case in its entirety, it comes into its own in the summer scenario. The perhaps surprisingly demanding search and rescue operations taking place in the summer months are not only highly complex, they also vastly outnumber interventions taking place in winter, of which avalanches only make up a small percentage, as the number of Swiss mountain rescue missions over the course of the year in Figure 1.4a show.

The majority of people rescued found themselves in an emergency situation while hiking. Activities such as mountain biking, climbing, or high mountain tours, are also common, reflecting the diverse recreational activities undertaken in the Alps. The leading cause for rescue missions is falling, followed by exhaustion, illness, or getting lost. Most people are uninjured or only slightly injured when they are rescued, however, life-threatening situations also can and do arise from these seemingly harmless emergencies [7, 8, 29].

In contrast to the winter scenario, rescue missions taking place during the summer months are characterized by a large search area, longer missions durations, and a higher degree of planning and coordination. Often the missing persons are in no immediate danger, but are lost in a vast area, leading to complex and very time consuming, large-scale search operations that can last several days [29]. Combined with the large number of missions and involved rescuers, this leads to an enormous amount 


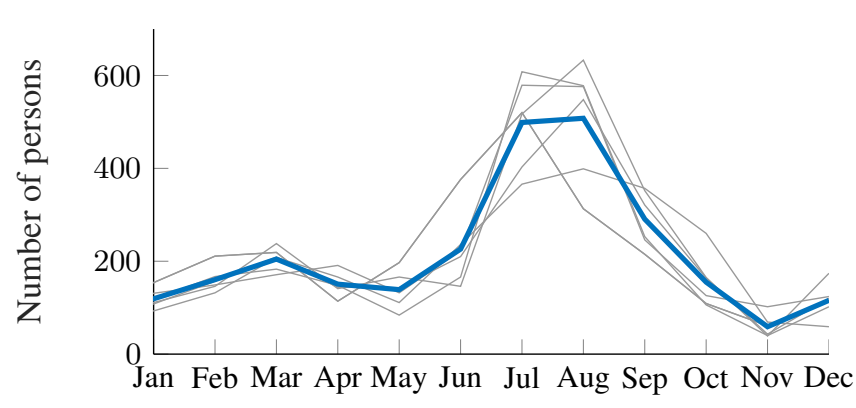

(a)

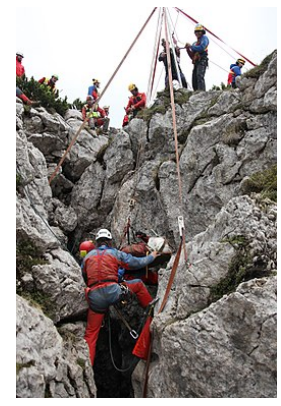

(b)

Figure 1.4: Number of persons rescued by Swiss mountain rescue organizations over the course of the year between 2011 and 2016 [8] (Figure 1.4a). The summer months show a substantially higher number of emergency, a trend which is also reported by other national and regional organizations [29]. Figure 1.4b shows a summer mountain rescue in highly adverse terrain (image courtesy of Bayerisches Rotes Kreuz, Kreisverband Berchtesgadener Land).

of working hours.

Usually teams of four to five rescuers search an area of several square kilometers by foot, and search for lost persons by sight. They may be accompanied by search dogs, or use mountain quads if possible, but they are often faced with very hostile terrain, including dangerous cliffs and overhangs. The coordination of the team, and staying focused during long missions are critical issues. Helicopters are highly effective above the tree-line, but cannot search forests, or fly at low altitude or in some adverse weather conditions. They are mainly used to transport equipment, and are very expensive to operate.

\subsubsection{Mission Outline}

The SHERPA project aims to support and coordinate the activities of human rescuers by introducing new technologies and platforms. The advantages of robotic systems become most clear when the search area is too treacherous or vast for humans, and the weather too bad for helicopters. We aim, however, to integrate the platform into existing procedures as far as possible for a smooth transition. Even moderate improvements can furthermore greatly reduce risks and discomfort for rescue personnel and victims, especially considering the scale of modern alpine rescue operations. A typical mission with the SHERPA platform in a summer scenario is outlined below. Even if the concept is scalable, with several SHERPA teams working together, we assume a mission involving a single team here.

The first members of the SHERPA team to begin the search operation are the hawks. They survey the search area, generate maps, and gather other information, while the rest of the operation is planned out. In a best case they may already localize the victims in this stage of the mission. Often emergency calls reach the rescuers in 
the evenings, when it is too late for humans to begin a search. In this case the hawks can still begin the operation at night.

In the next phase, the human rescuers arrive at the scene, along with the donkey and wasps. The busy genius commences to search the area by foot, supported by the wasps, acting as his eyes in the sky. The natural communication and autonomy of the system allows the busy genius to focus on the rescue operation, while the donkey follows him and services the wasps. Thanks to the robotic arm mounted on the donkey the wasps can be deployed and serviced without intervention of the busy genius, ensuring the system's endurance and autonomy.

The mission is concluded when the missing persons are found and brought to safety. In case of a search spanning several days, the robotic platforms could continue the search at night and capture thermal images or create maps of the search area autonomously without risking the safety of the busy genius.

\subsubsection{Requirements}

Some general requirements from the SHERPA project apply to all robotic agents, such as the ability to operate in adverse outdoor conditions. Another main feature of the SHERPA platform is autonomous operation, including coordination and planning of complex operations. A distributed delegation framework has been developed and implemented by the consortium for this purpose. Naturally, the ground rover and arm need to be interfaced with it, and integrated with the other agents. The most important case concerning the compliant manipulator is the coordination of the donkey, including the arm and service station, with the wasps for autonomous servicing.

Functional and hardware requirements pertaining specifically to the robotic manipulator are as follows:

- Reaching the landed wasp and its docking position on the donkey.

- Robust grasping of the wasp under misalignment.

- Lifting the wasp, including sensors and equipment, resulting in a nominal payload of approximately $2 \mathrm{~kg}$.

- Safe and dexterous manipulation of the wasps in an unknown and dynamic environment.

- Manageable system complexity.

- Easy transportation of the donkey to remote search areas. The arm must therefore be light and able to fold into a compact volume.

A tunable mechanical compliance can greatly increase the performance and safety of a dexterous manipulator in an unstructured environment, as outlined in the following Section. Such compliant actuation systems, however, usually increase the overall 
system complexity of a manipulator, which is why we have chosen to investigate a hybrid actuation scheme, combining traditional rigid and novel compliant actuators. In this way we can utilize the benefits of variable compliant manipulation, while keeping the costs limited. The compliant shoulder joint furthermore mechanically decouples the arm's inertia from the ground rover, which is an important feature to protect the manipulator from vibrations and impacts incurred when the rover is driving over rugged terrain.

\subsection{Robotic Manipulation}

Robotic manipulators have long established themselves in industrial applications such as welding, assembly, or packaging, thanks to their reliability, precision, speed, and strength. These classical industrial robots, however, cannot simply be applied to the SHERPA project, as they rely on a controlled and known environment, and cannot safely operate with humans or an unstructured environment. They are characterized by their fast and precise position control and high structural stiffness, and have been optimized for pick and place and trajectory tracking tasks. In order for robots to leave the factory floor and interact with different and new environments, including humans and unstructured outdoor settings, they need compliance to make them safe, adaptable, and robust.

\subsubsection{Compliant Actuation}

Compliant actuators are the core component of a next generation of robots, and have many important applications, including interactions with humans and the environment, as assistive $[30,31,32]$ or rehabilitation devices [33, 34], locomotion [35, 36, 37], and manipulation [38, 39, 40].

Compliance is either achieved actively through control (extrinsic compliance), or through passive compliant elements (intrinsic compliance). Active compliance is used more commonly, as it is mechanically simpler, only requiring a rigid connection between the motor's gearbox and the output link, but is limited by the performance of the controller and the inability to absorb impacts or store mechanical energy. Adding mechanical compliance can make actuators intrinsically safe and robust across all frequencies, not just in the controller's stable bandwidth.

SEAs (Series Elastic Actuators) [41] and VIAs (Variable Impedance Actuators) [42], in particular VSAs (Variable Stiffness Actuators) [43] are the most popular approach towards compliant actuation. Their intrinsic compliance provides several benefits over stiff actuators, most notably interaction safety [44, 45] and physical robustness [46], improved torque control performance and stability [47, 48, 49], and increased peak output power $[50,51]$ due to the ability to store mechanical energy. While the absorbed energy could also be transformed and recovered through regenerative braking, the most practical solution is the use of mechanical springs. This makes 
SEAs significantly more energy efficient [52], while the energy consumption of VSAs can be reduced even further by tuning the joint compliance to the natural frequency of a system when executing cyclic motions $[37,52,53]$.

\subsubsection{Collaborative Robotics}

The SHERPA project requires a robotic manipulator that executes a dexterous manipulation task in an unknown environment and can safely interact with humans and other objects. These requirements are very similar to those of a new, fast-growing class of robots. Collaborative robots, also called cobots, are intended to work alongside humans in a shared workspace, and to assist them in a variety of tasks. Because of their versatility, easy programming, and not least their affordability and focus on safety $[11,54]$, SMEs are eager to adopt this technology. Collaborative robots can save labour costs and increase efficiency, but also bring soft benefits like improved quality, and ergonomics and job satisfaction for the operator, and their global market is forecast to grow from 10.3 billion US dollars in 2015 to 95 billion US dollars by the end of 2024 [55]. Large manufacturers of classical industrial robots, such as FANUC, ABB, and KUKA, are expanding into the market of collaborative robots, where they compete with smaller specialized companies and start-ups, like Universal Robots, Rethink Robotics, or Franka Emika.

Universal Robots was one of the first companies to release a commercial collaborative robot in 2008. They now offer three models, the UR3, UR5, and UR10, and retain an important position in the market [56]. In 2012 Rethink Robotics introduced Baxter, a dual manipulator robot using SEAs, and more recently the one armed robot Sawyer $[57,58]$. Kinova's $\mathrm{JACO}^{2}$ manipulator is an example showing that collaborative robots not only find applications in industrial settings, but can also be used as assistive devices for people with disabilities [59]. One of the major robotics providers, FANUC offers the strongest collaborative robots available on the market with a payload of up to $35 \mathrm{~kg}$ in their CR series [60]. Launched in 2013, KUKA's LBR iiwa is the result of a close collaboration with the German Aerospace Center (DLR), which already had developed several generations of torque-controlled light weight robots, and is capable of very sensitive torque and impedance control [61]. Franka Emika's newly introduced Panda robot is a close derivative of the LBR iiwa, with a smaller payload but similar performance. However, with its low price of only 10.000 euro, a fraction of the cost of other manipulators, it has the potential to shake up the industry [62]. Other commercial collaborative manipulators include ABB's YuMi [63], Barrett Technology's WAM arm [64], F\&P Personal Robotics' P-Rob 2 [65], Bosch's APAS assistant [66], Yaskawa's Motoman HC-10 [67] Comaus AURA [68] arm, and Stäubli's TX2 line [69].

All of these robots are designed to work alongside humans without safety barriers separating the two, and must therefore be safe for human robot interaction. Two factors must be considered for safety: the collision forces generated during free impacts, and the contact forces occurring after constrained contact has been established. Both 


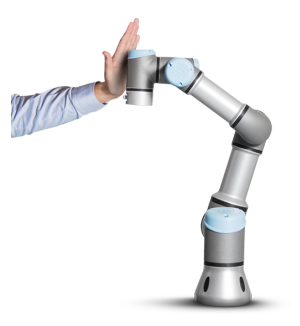

(a) Universal Robots UR3

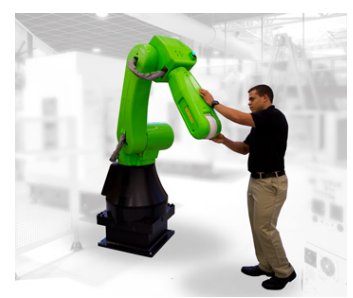

(d) FANUC CR-35iA

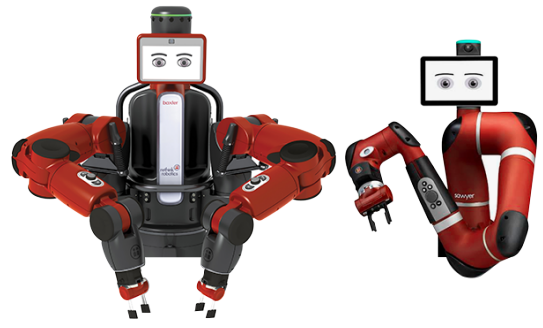

(b) Rethink Robotics Baxter \& Sawyer

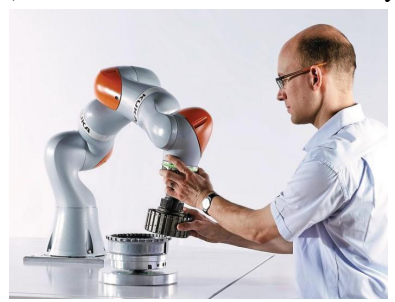

(e) KUKA LBR iiwa

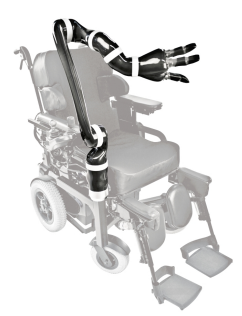

(c) Kinova $\mathrm{JACO}^{2}$

Figure 1.5: Collaborative industrial robots capable of sharing their workspace and safely interacting with humans. Of these robots only Rethink Robotic's Baxter is equipped with SEAs, which makes it intrinsically safe.

can be viewed from an energetic perspective; the impact forces relate to the kinetic energy dissipated at a collision, and thus to the mass and speed of the moving manipulator. The static contact forces the robot inflicts on humans or the environment are linked to the the potential energy accumulated in the robot's compliant structure.

The revised EN ISO 10218 standard Parts 1 and 2, and the ISO/TS 15066 specification define safety requirements for collaborative robots, describe the biomechanical limits for pressures and forces the human body can absorb, and offers guidance for the process of risk assessment [54, 70, 71, 72].

Some robots limit their performance in terms of maximum velocities, forces, and power for safety $[65,59,67]$, but in order to effectively cooperate they need to detect and react to contacts, either through measuring the joint torques [61, 62, 67], or through additional sensors on the robot's surface [73], the latter often in combination with soft protective covers that absorb impacts and avoid pinching [60, 63, 65, 68], or even through contact-free sensing [66]. Reacting to the environment in a compliant way is also essential for dexterous manipulation in an uncertain environment $[39,74]$. Intrinsic safety, however, can only be achieved by mechanically decoupling the load from the actuators, through slip clutches or backdrivable joints [59, 64], or through physically compliant joints $[57,58]$.

While the state of the art for safe and dexterous manipulation in the industry is actively controlled impedance with rigid robots, Baxter and Sawyer being the only com- 
mercial robots equipped with SEAs, intrinsically compliant actuation is more common in research oriented platforms, such as NASA JSC's Valkyrie (R5) [75], WALKMAN [76], or the bi-manual platform developed for the CENTAURO project [77]. As already noted, the addition of physical springs has important implications not only for the dexterity of a system, but also its energy efficiency, which is especially relevant for mobile robots. Platforms equipped with SEAs can temporarily store potential energy and exploit their passive dynamics. Platforms designed around VSAs can moreover tune their passive compliance, making them adaptable to different tasks and changing environments. Owed to their high system complexity, only very few variably compliant manipulators exist, such as the MIA Arm [78] or David (formerly the DLR Hand Arm System) [79], and none are available as off the shelf components. However, their large potential benefits, and promising performance $[79,80,81]$, make them highly desirable.

\subsection{Research Objective}

The objectives of this dissertation can be divided into scientific research objectives, and more practically oriented engineering objectives associated with the analysis, design, construction, control, and integration of a robotic manipulator for use in the SHERPA project. Compliant actuation is crucial to the required interaction tasks in unstructured environments, as it implicitly controls the energy exchange with the environment. Intrinsic compliance hereby offers several fundamental advantages over merely controlled compliance. As intrinsically compliant robotic manipulators are not yet readily available on the market, we have used this opportunity to address several open research questions pertaining to this dynamic and important field, next to the topics relating to heterogeneous multi-robot and human-robot interactions investigated in the SHERPA project. The research presented in this dissertation was hereby guided by research objectives (ROs), that are defined here as open scientific problems necessary for successfully meeting the project goals. These objectives are formulated as the following questions:

- RO1 How is energy exchanged with the environment absorbed by VSAs and routed to their internal energy storage; which types of VSAs best utilize their internal springs?

Physical interaction with the environment implies an exchange of physical energy that is exorted or absorbed by the manipulator's VSAs, and ideally stored in its internal springs. A large number of VSA designs have been implemented over the last years, however, they do not all achieve their variable output stiffness in the same way, just as the degree to which they can utilize their springs can differ vastly.

- RO2 How can we maximize the energy storage capacity of the VSAs' internal springs? 
Despite the potential benefits of this technology, VSAs have not yet been implemented in many real-world applications. One of the design bottlenecks is the ability of the mechanical springs to store enough elastic energy, while fitting into a compact volume.

- RO3 How should the actuation topology of rigid-compliant hybrid systems be devised, such that we can achieve a desired range of workspace compliances with a minimal number of VSAs?

Another drawback of VSAs is their higher complexity compared with traditional actuators. We investigate to what extent we can retain the advantages of variably compliant joints, when placing as few VSAs as possible, and thus limiting the additional system complexity.

- RO4 How can we best utilize the resulting workspace compliance for manipulation tasks, and formulate corresponding control problems?

Even a manipulator with variable compliance in every joint cannot achieve an arbitrary workspace compliance. A sound mathematical basis is needed to describe the achievable forces and motions of the arm.

- RO5 How can complex collaborative tasks involving several robotic or human agents be formulated, delegated, and executed?

In complex autonomous mission, for instance as envisaged by SHERPA, robotic agents need to be able to plan and formulate collaborative tasks, divide them among themselves, and execute them in a cooperative fashion.

In addition to investigating these scientific research objectives, the following engineering objectives (EOs) need to be fulfilled. These define the practical engineering tasks that are necessary to accomplish the project's implementation requirements and to provide the means to answer the scientific research objectives.

- EO1 Determine manipulator kinematics such that all critical points in the workspace, such as the landed UAV and the docking position, are reachable and manipulable, while the arm can fold into a compact volume.

- EO2 Design and construction of the arm's components, including joints, VSAs, gripper, and sensors. Integration of components, control, and testing of the manipulator.

- EO3 Ensure robust grasping of the UAV under misalignment and unknown ground surface.

- EO4 Integration of the manipulator with the SHERPA platform and control framework, demonstration of the arm's servicing tasks and the effectiveness of the system. 


\subsection{Contribution}

The major contribution of this work is the design and operation of the manipulator developed according to the goals stated in Section 1.3. This manipulator helped to demonstrate the project's feasibility and thus contributed to the application of robotics for search and rescue operations in particular, and the promotion of autonomous service robotics in general. The work can also be applied to human-robot interaction, notably compliant physical interaction.

The thesis' specific contributions with respect to the research objectives are:

- RO1 A lever-arm based VSA design was chosen that changes its output stiffness by changing the transmission between its internal springs and output, thus preserving its energy storage capacity over a wide stiffness range.

- RO2 An $\Omega$-shaped polymer leaf spring optimally uses the available mounting volume and maximizes the energy storage capacity according to a detailed elastostatic analysis of geometric and material properties. This work was published in [82].

- RO3 It was discovered that the desired range of workspace compliance is already achievable with three joints with variable compliance; one VSA in the shoulder, and two joints with a coupled variable stiffness in the wrist, as published in [83].

- RO4 Using screw theory, the forces and motions of the manipulator's endeffector are described as twists and wrenches. These can be projected into the compliant subspace of the workspace for a given configuration of the robot.

- RO5 High-level control operations of the arm are formulated in task specific trees, a hierarchical task description that can integrate heterogeneous agents and several layers of abstraction. The coordination of these agents is performed within a powerful delegation framework, auctions tasks according to agent's resources and capabilities. This work was published in [84].

The engineering contributions are:

- EO1 The manipulator is constructed as a roughly anthropomorphic 7-DoF robotic arm, with spherical 3-DoF shoulder and wrist joints, a unique elbow design that allows the arm to fold into a compact volume, and reaches all required poses. The mechatronic design of the manipulator is published in [83].

- EO2 A considerable amount of design, testing and integration work has been carried out on both the component and system level, resulting in a working manipulator for use in the SHERPA project, and as research platform. The inclusion of novel VSAs, and their efficient application are of particular interest. 
- EO3 A custom gripper was designed that engages an interface mounted on the UAV, and achieves a secure form closure by exploiting the arm's compliance, and shows a robust and safe performance. The design of the gripper was published in [85].

- EO4 The viability of mechanically compliant manipulation for autonomous interaction and servicing tasks, and the proposed collaborative search and rescue mission was demonstrated through integrated experiments with the SHERPA system, as published in [84].

\subsection{Thesis Outline}

A detailed classification and a port-based approach to the modeling and analysis of VSAs is presented in Chapter 2, and used to answer the research objective RO1. RO2 is also addressed in this chapter through the analysis and design of the VSAs' internal springs.

The actuation topology and manipulator kinematics are derived in Chapter 3 after analyses of the workspace and the arm's end-effector compliance, answering RO3 and EO1. The results of the engineering objective EO2 are also treated here by detailing the design and integration of the manipulator's components up to a demonstration of the working system.

Chapter 4 presents the design of the gripper that enables robust grasping of the UAV as required by EO3.

Chapter 5 provides a rigorous mathematical basis for investigating how to properly exploit the compliant joints, which answers RO4 and can be used to address RO3.

Chapter 6 presents the autonomous battery exchange operation carried out with the arm, demonstrating the compliant manipulator's capabilities, and underlines the role such automated systems can play in supporting and improving search and rescue operations. RO5 and EO4 are addressed through the autonomous planning and execution of the task, and the integration of the manipulator within the SHERPA system.

Concluding remarks are made in Chapter 7. 



\section{Elastic ENERGy STORAGE IN LEAF SPRINGS FOR A LEVER-ARM BASED VARIABLE STIFFNESS ACTUATOR}

Robots physically interact with their environment by applying forces to move or manipulate objects. This requires mechanical work and the exchange of energy. To make these interactions safe and energy efficient modern robots are equipped with elastic elements to absorb and store mechanical energy. Actuation systems like SEAs (Series Elastic Actuators) and VSAs (Variable Stiffness Actuators) include internal springs for this purpose, but their energy storage capacity is often still a bottleneck for the robot's performance, as these actuators need also to be light and compact. This chapter presents a port-based model to analyze the power flow inside VSAs, and to select a suitable design that uses the internal springs in such a way, that the energy capacity is not impaired by adjusting the stiffness setting, hereby addressing this dissertation's research objective RO1. Design guidelines for the internal springs of a lever-arm based VSA are then derived and the design of a polymer leaf spring is presented and experimentally validated, answering RO2.

Parts of this chapter were previously published as:

E. Barrett, M. Fumagalli and R. Carloni, "Elastic energy storage in leaf springs for a lever-arm based Variable Stiffness Actuator,' 2016 IEEE/RSJ International Conference on Intelligent Robots and Systems (IROS), pp. 537-542, 2016. 


\subsection{Introduction}

The adoption of compliant joints is helping robots intended for interaction and collaboration with humans and unknown dynamic environments to meet the demands associated with these tasks. Robots and their actuators need to be intrinsically safe, compact and light weight, and energy efficient, while still being able to absorb sufficient amounts of energy during interactions with the environment. The compliant elements in the joints absorb impact forces, leading to increased safety for both the robot and its environment [86], while reducing the required control effort by embodying a desired behavior in the system's natural dynamics [87], store mechanical energy, increase energy efficiency [86], and the actuator's peak output power [88].

VSAs are capable of changing their output stiffness, which makes them suited for a wide range of tasks and enables them to tune their natural dynamics [37, 52, 53]. A large number of different VSAs have been presented in the recent years [42], which can be grouped by the way they achieve a variable output stiffness, namely by adjusting the spring preload $[89,90]$, adjusting the transmission between spring and load [91, 92, 93], or adjusting the physical properties of the spring [78, 94].

Independent of their operating principle, however, all VSAs require internal springs to store mechanical energy during interaction. The design of which is a trade-off between the weight and size of these springs on the one hand, and their energy storage capacity on the other. Given the high requirements in terms of weight and compactness, as well as output loads and energy storage, the proper selection of the spring type, material, and dimensions, is of great importance for a large torque-deflection workspace. Due to the many variables involved, the design of elastic elements is often based on heuristics, even though a structured design approach can greatly improve the springs' performance, as shown in [95].

While new performance measures of this novel class of actuators are still being developed [96], the stiffness range, energy absorption capacity, and torque deflection workspace are directly related to the capacity and usage of the actuators' internal springs.

The contribution of this chapter lies in the analysis of the effect that the selection and design of the VSA mechanism, and the internal springs has on the performance of lever-arm based VSAs, in particular on the energy storage capacity, which is limited by the compactness of the system. The working principle and requirements for leverarm based VSAs are presented, along with a design methodology that maximizes the energy storage for such actuators, leading to the development of a novel polymer leaf spring. This methodology is based on an analysis of the relations between the spring parameters and validated by simulation and experimental results on the variable stiffness mechanism shown in Figure 2.11.

This chapter is organized as follows. Section 2.2 classifies different VSA designs and presents a port-based model to aid in the evaluation of the designs, which is carried out in Section 2.3. Section 2.4 examines the particular design requirements for 
lever arm based VSAs and provides basic guidelines and requirements for the internal springs. The design methodology for the chosen leaf springs is discussed in detail in Section 2.5. The results of the FEA (Finite Element Analysis) are experimentally validated in Section 2.6, before the results are discussed in Section 2.7, and concluding remarks are made in Section 2.8 .

\subsection{Classification and Modeling of VSAs}

Many different VSA designs, utilizing different operating principles, have been presented over the last years. This section aims to give a brief overview and classification, before presenting a port-based model that is employed to describe and analyze this class of actuators.

\subsubsection{Operating Principles}

It is helpful to classify VSAs in terms of their operating principle in order to gain a better overview and understanding of how they adjust their output stiffness and exploit their internal springs, and what kind of performance can be expected from them. While a detailed review of VSA designs can be found in [42], a general categorization according to VSA's basic modes of operation is given below.

\section{Adjusting the Spring Preload}

VSAs of this class adjust their output stiffness by changing the pretension of their internal springs. These springs either are non-linear springs, or linear springs in combination with a non-linear transmission between springs and output, but rely on pretensioning the springs to change their force-deflection characteristic. Subcategories of this class utilize antagonistic springs with antagonistic motors [37, 97, 98, 99], antagonistic springs with independent motors that decouple stiffness and position control [100], or adjust the preload of a single spring through a non-linear connection to the output $[52,89,90]$

\section{Adjusting the Transmission between Spring and Load}

The transmission between the internal springs and the output is altered, changing how the springs are felt at the load. Subcategories include variable lever arm ratios, nonlinear mechanical interlinks, and continuously variable transmissions $[92,101,93,102$, 103, 104, 105, 106].

\section{Adjusting the Physical Properties of the Spring}

VSAs of this category alter the physical structure of their internal springs. For instance by changing the active length of a leaf spring [78, 94], or the number of active coils of 
a helical spring [107]. Even though the focus of research has recently shifted to other types of VSAs, this class may see more attention in the future when novel materials that can change their mechanical stiffness become available.

\subsubsection{Port-based Model}

Figure 2.1 shows a simple example of a one-dimensional controlled system, where an actuated mass $m_{a}$ with position $a$ is connected through a spring and damper with a mass $m_{y}$ with position $y$, on which the environment acts with the force $F_{y}$. This model can be seen as a SEA, with an interaction port [108] for control, and one for the environment, as well as internal energy storage and dissipation. Next to the ideal physical model, Figure 2.1 also shows the bond graph representation [109, 110] of the system.

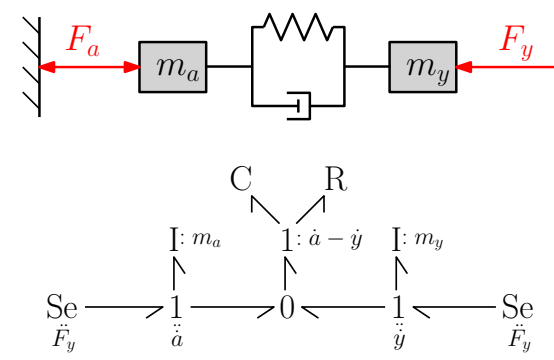

Figure 2.1: A simple controlled system as ideal physical model, and in bond graph notation. The controlled mass $m_{a}$ is connected to the load $m_{y}$ through a spring and damper. Their corresponding inertias are connected to one-junctions, representing the flows (velocities) $\dot{a}$ and $\dot{y}$, the $\mathrm{C}$ and R-elements, representing the spring and damper respectively, are connected to a one-junction representing the difference of velocities between actuators and load, which is expressed in the zero-junction, representing the shared force. The interaction forces $F_{a}$ and $F_{y}$ are represented as effort sources.

Using the approach of connecting elements that exchange energy through ports makes bond graphs an intuitive and versatile tool, based on the actual physical behavior of the system. The individual elements of the model are connected through power bonds consisting of effort and flow variables, which form the space of power variables $\mathcal{F} \times \mathcal{F}^{*}$ on the linear space of flows $\mathcal{F}$, and the dual space of efforts $\mathcal{F}^{*}=: \mathcal{E}$. Usually the space of flows is simply defined as $\mathbb{R}^{n}$, however, in some cases other choices have their advantages. In Chapter 5, for instance, the spaces of twists $\mathcal{F}=s e(3)$ and wrenches $\mathcal{E}=s e^{*}(3)$ are used for a coordinate free description of rigid body motions. Figure 2.1 contains one-dimensional single bonds, with $n=1$; the remainder of this introduction, however, assumes the general $n$-dimensional multi bond case, which is denoted by a double line. Examples of power variables are pairs of forces, voltages, or pressures and their corresponding velocities, currents, or volumetric flow rates, respectively. The power of a pair of effort and flow variables is defined by the duality product $P=\langle e \mid f\rangle$, while its positive direction of the power flow is denoted by the 
direction of the half-arrow:

$$
\stackrel{e}{\rightleftharpoons} \quad\langle e \mid f\rangle:=e(f)=P ; \quad e \in \mathcal{E}, \quad f \in \mathcal{F}
$$

Several multi-port energy-routing elements exist, that define the power flows within the model. A power continuous connection of multiple elements is represented through a one, or a zero junction. All bonds connected to a zero junction share the same effort, while the sum of their flows is zero. Bonds connected to the dual one junction, share the same flow, while the sum of efforts is zero.
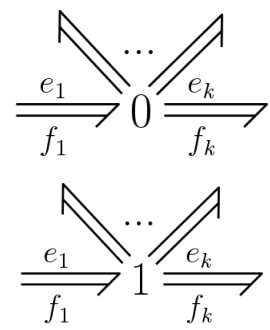

$$
\begin{aligned}
& e_{1}=e_{i}, \quad \sum_{i=1}^{k} f_{i}=0 \\
& f_{1}=f_{i}, \quad \sum_{i=1}^{k} e_{i}=0
\end{aligned}
$$

Equation 2.4 represents an ideal transformer, that relates the efforts and flows of both of its ports by the transformer ratio $\alpha \in \mathbb{R}^{n \times m}$, where $n$ and $m$ are the dimensions of the effort and flow vectors at the two ports of the element. Examples of transformers are mechanical transmissions like gears or levers, or electrical transformers. The dual of a transformer is a gyrator, which relates efforts to flows and vice versa. One example of such a device is a DC electric motor, which relates electric current into mechanical torque, and velocity into counter-electromotive force.

$$
\begin{aligned}
& \stackrel{e_{1}}{\rightleftharpoons} \mathrm{TF} \stackrel{e_{2}}{\stackrel{f_{2}}{\rightleftharpoons}} \quad e_{1}=\alpha^{T} e_{2}, \quad f_{2}=\alpha f_{1} \\
& \stackrel{e_{1}}{\stackrel{f_{1}}{\rightleftharpoons}} \mathrm{GY} \stackrel{e_{2}}{\stackrel{f_{2}}{\rightleftharpoons}} \quad e_{1}=\alpha^{T} f_{2}, \quad e_{2}=\alpha f_{1}
\end{aligned}
$$

Modulated transformers and gyrators (MTFs and MGYs) have variable transformation or gyration ratios $\alpha$, that can be controlled by external variables, which can for instance be generalized positions or velocities.

It is easily verified that the elements introduced so far are all power-continuous, which means that the algebraic sum of the powers $P_{i}=\left\langle e_{i} \mid f_{i}\right\rangle$ entering and exiting through the connected ports is zero. There are two dual free energy ${ }^{1}$ storage elements, $\mathrm{C}$ and I-type elements, based on the generalized displacement $q$ and the generalized momentum $p$, respectively, which are the state variables of the system. The C-type element stores generalised potential energy, like in a mechanical spring or electrical capacitor, by integrating the flow to obtain the generalized displacement $q$.

\footnotetext{
${ }^{1}$ The thermodynamic free energy is that energy which can be used to perform work, in contrast to the unusable energy given by a system's entropy and temperature.
} 


$$
\stackrel{e}{=} \mathrm{C} \quad q(t)=\int_{0}^{t} f(t) d t, \quad e=\frac{\partial H(q)}{\partial q}
$$

where $H(q)$ represents the total stored potential energy of the storage element.

The I-type element integrates effort, to obtain the generalized momentum $p$. Examples of this are mechanical masses and electric inductors.

$$
\stackrel{e}{\rightleftharpoons} \mathrm{I} \quad p(t)=\int_{0}^{t} e(t) d t, \quad f=\frac{\partial H(p)}{\partial p}
$$

Free energy is dissipated through irreversible transformers, the resistive $\mathrm{R}$ elements. The effort exerted by these elements is a function of the flow or vice versa.

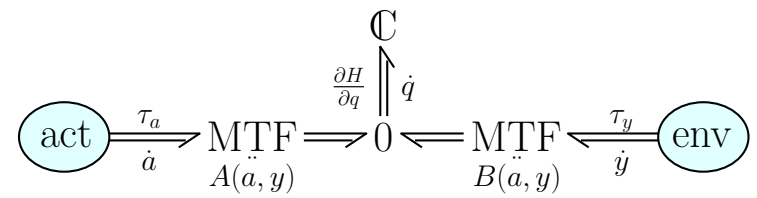

Figure 2.2: Port based model of a generic VSA. The actuators and the environment are connected to the internal energy storage element through modulated transformers.

While bond graphs are an excellent graphical tool to model dynamic systems based on their physical power flows, port-Hamiltonian systems [108] offer a more analytical method to describe physical systems in an energy consistent way. After introducing the concept of power ports and the bond graph notation by means of a simplified model for SEAs, a more general port-Hamiltonian model for VSAs is given in Figure 2.2. In this particular model all energy storage is concentrated into a single C-type element, connected to the storage port. The I-type storage, i.e. the inertia of the stiffness change mechanism, is often neglected, but can also be included in the C-type element through the generalized bond graph approach [111] by using a symplectic gyrator, which is a gyrator like explained above, but specifically with identity transformation, to transform the I-type storage to a dual C-type element. Energy dissipation is not considered here, as the focus lies on how the energy is routed between the ports of the model, but can be easily added by including a dissipation port connected to an R-element.

As was noted above, a number of power continuous elements exist that route energy, but do not store or dissipate it. When such elements are interconnected that property remains, and the total algebraic sum of the power flowing through their ports is still zero. This leads to a central concept in port-Hamiltonian modeling, which is that of Dirac structures $[112,113]$, which basic property is power conservation. It links various ports in such a way, that the total power $\sum P_{i}=\sum\left\langle e_{i} \mid f_{i}\right\rangle$ of connected ports $i$ passing through it, as defined in Equation 2.1, is zero, i.e. there is no energy stored or dissipated. The modulated transformers and 0 -junction in model 2.2, defining the power flows between the internal actuators, the spring element, and the 
environment, are such a Dirac structure, described by the following matrix Equation (2.8) [114].

$$
\left[\begin{array}{c}
\dot{q} \\
\tau_{a} \\
\tau_{y}
\end{array}\right]=\left[\begin{array}{ccc}
0 & A(a, y) & B(a, y) \\
-A^{T}(a, y) & 0 & 0 \\
-B^{T}(a, y) & 0 & 0
\end{array}\right]\left[\begin{array}{c}
\frac{\partial H(q)}{\partial q} \\
\dot{a} \\
\dot{y}
\end{array}\right]
$$

It can be seen from Equation (2.8) that in the absence of a gyroscopic coupling between forces and velocities, which indeed does not exist in the mechanical domain, $A(q, y)$ and $B(q, y)$ define the contribution of the actuator and output flows to the rate of change of the spring state $\dot{q}=\frac{\partial q}{\partial a} \dot{a}+\frac{\partial q}{\partial y} \dot{y}$, and are thus the partial derivatives of the spring state $q(a, y)$ :

$$
A(a, y)=\frac{\partial q(a, y)}{\partial a}, \quad B(a, y)=\frac{\partial q(a, y)}{\partial y}
$$

Furthermore, Equation (2.8) gives the output torque as

$$
\tau_{y}(a, y)=-B^{T}(a, y) \frac{\partial H}{\partial q}
$$

In this scalar case the stiffness, which relates the generalized forces to the generalized displacements, can be defined as the partial derivative of the output torque with respect to the position

$$
K(a, r)=\frac{\partial \tau_{y}(a, y)}{\partial y}
$$

It should be noted that all the above expressions characterizing a VSA can be derived from the spring state $q(a, y)$. Likewise, the energy stored in the system is defined by the springs' energy function $H(q)$. The energy can be supplied by the actuators through the control port, or by the environment through the output port. How it is routed, and the springs utilized, depends on the Dirac structure, which makes this model particularly suited to analyze the energy storage capabilities of generic VSAs.

\subsubsection{VSA Designs}

This section presents four particular VSA designs, based on different operating principles in the port-based framework introduced above. Though far from exhaustive, these examples give a good overview of the state of the art in VSAs, and represent some of the most common designs. With the presented model we can investigate how the energy storage capabilities of these VSAs relate to their stiffness change capabilities and how they utilize their internal springs. A lever arm mechanism, representing a variable transmission operating principle, the MACCEPA (Mechanically Adjustable Compliance and Controllable Equilibrium Position Actuator), the FSJ (Floating Spring Joint) mechanism, and an antagonistic VSA are selected for this comparison. While the MACCEPA and FSJ mechanism both change the preload of a single spring, they still 


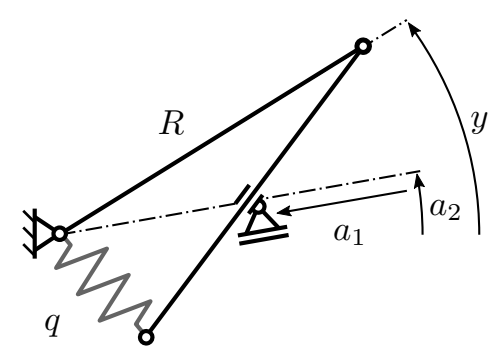

Figure 2.3: This VSA design is based on the way a deflection of the lever on its output side, caused by a rotation of the crank $R$, causes a deflection of the spring, which is attached to the other side of the lever, depending on the lever arm ratios. The position $a_{1}$ of the pivot point hereby determines the transmission between the output angle $y$ and the spring state $q$, where the output and equilibrium positions $y$ and $a_{2}$ respectively are measured with respect to a global reference.

represent very different designs. Analogous to the human arm with biceps and triceps, the simple antagonistic VSA demonstrates the most basic, and nature inspired approach to variable impedance actuation, and the lever-arm mechanism represents the large class of VSAs based on a variable transmission.

The following overview will present the mechanisms' spring states $q(a, y)$, which, together with the springs' energy function $H(q)$, fully characterize their behavior, as shown above. Other characteristics such as the output torque or stiffness can be derived from these in a straight forward way.

\section{Lever Arm Mechanism}

A number of VSAs adjust their stiffness with a lever arm mechanism that changes the transmission between the spring and the output [91, 92, 93, 101, 102]. In this case the lever connects the output on one end to a spring on its other end, while it is free to move around its pivot point. It was shown that the best option of changing the lever arm ratios is to move the pivot point along the lever, [91] as in the present case. A sketch of the mechanism is shown in Figure 2.3, from which the spring state can be derived as

$$
q(a, y)=\left(\frac{R^{2}}{a_{1}}-R\right) \sin \left(y-a_{2}\right)
$$

\section{MACCEPA}

The mechanism realized by MACCEPA [90] uses a single linear spring, which connects an actuated link that determines the equilibrium position with a second link that is connected to the load, as shown in Figure 2.4. The stiffness is changed by moving the attachment point of the spring along the output link during which the pretension of the spring is changed. A sketch of the mechanism is shown in Figure 2.4 and the spring 
state $q(a, y)$ given in Equation 2.13. In a variation of the mechanism [52] the actuated link is equipped with a profile disk, such that the torque-deflection characteristic can be modified.

$$
q(a, y)=\sqrt{a_{1}^{2}+R^{2}-2 a_{1} R \cos \left(y-a_{2}\right)}
$$

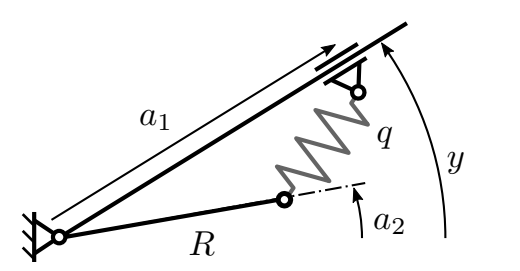

Figure 2.4: The equilibrium position of the MACCEPA mechanism is controlled through the position $a_{2}$ of a lever arm with length $R$. It is connected to the output link through a spring, which is pretensioned according to the position $a_{1}$. Increasing the pretension will increase the torque the spring exerts on the output $y$ when it is not aligned with the lever $R$ where the output and equilibrium positions $y$ and $a_{2}$ respectively are measured with respect to a global reference.
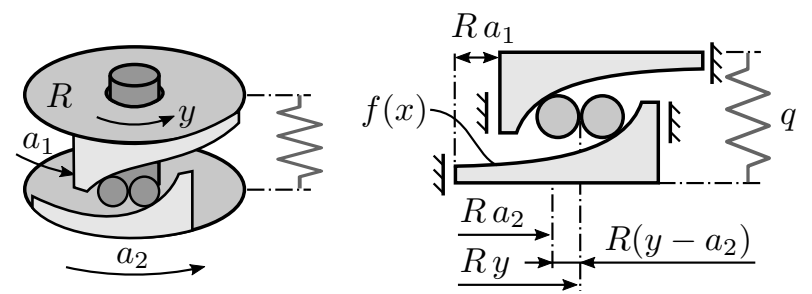

Figure 2.5: The floating spring joint (FSJ) consists of two cam disks of radius $R$ and profile $f(x)$, held together by a 'floating' spring in the center of the assembly. The distance of the disks is determined by the position of the cam rollers on the (exponential) cam profile, thus creating a non-linear transmission between angular displacement of the disks, and the spring state $q$. The main actuator $a_{2}$ is connected to one of the disks, while the second actuator $a_{1}$ pretensions the spring. The output $y$ is attached to the cam rollers.

\section{Floating Spring Joint}

Like the VS-Joint [115], the FSJ [89] uses a cam profile to translate an angular displacement of a roller into a linear displacement of a linear spring through a non-linear transmission. Unlike the VS-joint, however, the FSJ uses two cam disks placed above each other, as shown in Figure 2.5. When the output is deflected, one roller will move 'up' the cam profile, extending the spring, while the other will move 'down', such that the spring state is

$$
q(a, y)=f\left(\frac{a_{1}}{2}+\left(y-a_{2}\right)\right)+f\left(\frac{a_{1}}{2}-\left(y-a_{2}\right)\right)-2 f(0)
$$


In order to achieve a progressive spring state and stiffness, the function $f$ describing the cam profile must also be progressive. To change the stiffness of the actuator, the two cam profiles are rotated against each other, which pretensions the spring.

For an exponential cam profile $f(x)=\frac{x_{0}}{2} e^{\alpha x}, x_{0}$ can be chosen such that the spring is maximally extended to $q_{\max }$ at maximum deflection with zero pretension $a_{1}=0$, thus $x_{0}$ is given as $x_{0}=\frac{2 q_{\max }}{\exp \left(\alpha R\left(y_{\max }-a_{2}\right)\right)+\exp \left(-\alpha R\left(y_{\max }-a_{2}\right)\right)-2}$ while $\alpha$ can be used to shape the torque profile and stiffness range. The maximum pretension that can be achieved is then $a_{1 \max }=2 \frac{\log \left(q_{\max } / x_{0}+1\right)}{\alpha R}$.

The spring state becomes

$$
q(a, y)=\frac{x_{0}}{2}\left(e^{\alpha R\left(a_{1} / 2+\left(y-a_{2}\right)\right)}+e^{\alpha R\left(a_{1} / 2-\left(y-a_{2}\right)\right)}-2\right)
$$

where $a_{2}$ needs to be adjusted as $a_{2}=-\frac{a_{1}}{2}$ to not change the equilibrium position when the actuator's stiffness preset is changed.

\section{Uni-directional Antagonistic}

A uni-directional antagonistic VSA design with independent actuators [37, 97], as shown in Figure 2.6, is chosen for this analysis. This is the most basic antagonisitc VSA design and uses the simplest spring setup. Even though the cross-coupling antagonistic design [98] alleviates the preload on the actuators, another pretensioned spring is needed for this purpose. Similarly, the bidirectional antagonistic design [99] uses a pair of non-linear springs on each actuation unit, thus increasing the complexity and number of parts. Here, two actuators are connected to the output pulley via nonlinear springs. These springs may be intrinsically progressive [97], or linear springs connected through a non-linear mechanism $[99,100]$. They may have various characteristics, but are here assumed to be quadratic tension springs with the energy function $H(q)=\frac{1}{3} k q^{3}$, as in this case the output stiffness only depends on the springs' pretension, and not on the output deflection. The state (deformation) of the springs is

$$
q(a, y)=\left[\begin{array}{l}
a_{1}-y R \\
a_{2}+y R
\end{array}\right]
$$

Note that $q$ is two-dimensional and the springs can be deflected independently of each other. The springs furthermore need to be pretensioned to half their designed elongation $q\left(a_{1}=a_{2}=y=0\right)=R y_{\max }$ for one of the springs to reach zero pretension at maximum deflection of the output.

\subsection{Design Evaluation}

This section evaluates the previously presented VSA designs by means of the portbased model presented in Section 2.2.2. In particular it is investigated if energy is captured in the internal springs when the stiffness is changed through the actuation 


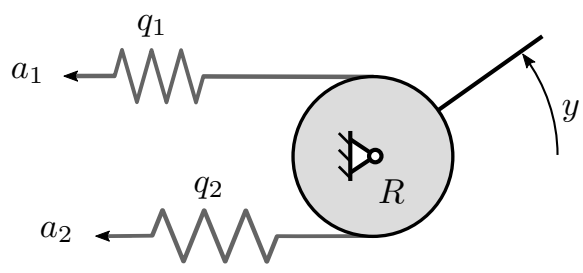

Figure 2.6: Two actuators $a_{1}$ and $a_{2}$ are connected to a pulley with radius $R$ through two non-linear springs $q_{1}$ and $q_{2}$. Moving both actuators in the same or opposite directions either controls the pretension of the springs, thereby changing the stiffness, or moves the equilibrium position of the actuator, where the output position $y$ is measured with respect to a global reference.

port, thus reducing the remaining energy storage capacity that can be used during interaction with. And secondly, how much energy a VSA can absorb from the environment as a function of its stiffness setting.

\subsubsection{Energy Efficient Actuation}

The analysis of the structure of the port-based model can be used to investigate the internal power flows of a VSA. In terms of energy efficient actuation it can be determined how much energy that is supplied by the actuators actually reaches the output of the system, and which ratio of it is captured by the internal springs [116]. Similarly [117] analyzed if a stiffness change of a particular VSA, requiring a change of the actuator positions $a$, also entails a change of the energy stored in the internal elastic elements, and found that this was not the case for lever arm based designs. These findings are not only relevant for the actuation of VSAs, but also for the utilization of the energy storage, when this capacity is lost due to pretensioning of the internal springs.

The map $A(a, y): T_{a} \mathcal{A} \rightarrow T_{q} \mathcal{Q}$ maps actuator flows $\dot{a}$ to changes in the configuration of the energy storage elements $\dot{q}$. If this map has a kernel, or null space, of dimension $\operatorname{dim}(\operatorname{ker} A(a, y))>0$ it follows that trajectories $\dot{a}$ exist, such that $\dot{q}=0 \Rightarrow \dot{H}(q)=0$ if $\dot{a} \in \operatorname{ker} A(a, y)$ [118]. We can show that the matrix $A(a, y)$ for the lever arm mechanism, obtained by differentiating the spring state $q$ in Equation 2.12 , as

$$
A(a, y)=\frac{\partial q}{\partial a}=\left[-\left(\frac{R}{a_{1}}\right)^{2} \sin \left(y-a_{2}\right), \quad R\left(1-\frac{R}{a_{1}}\right) \cos \left(y-a_{2}\right)\right]
$$

has a null space $\operatorname{null}(A)=\left\{\dot{a} \in T_{a} \mathcal{A} \mid A(\dot{a})=0\right\}$ spanned by a solution to $A(a, y) \dot{a}$ :

$$
\operatorname{null}(A(a, y))=\operatorname{span}\left\{\left[1, \quad \frac{R \tan \left(y-a_{2}\right)}{a_{1}^{2}-R a_{1}}\right]^{T}\right\}
$$

If the actuator positions are thus controlled in such a way that they lie within the null space of $A(a, y)$, we can change the stiffness setting of the actuator, without 
changing the deflection of and energy stored in the springs. Note that if the output deflection $y-a_{2}$ is zero $a_{1}$ can move freely without deflecting the spring, while the equilibrium position $a_{2}$ needs to be adjusted when the output is loaded.

The MACCEPA mechanism also permits null space motions which can adjust the stiffness without further elongating the spring

$$
\operatorname{null}(A(a, y))=\operatorname{span}\left\{\left[a_{1} R, \frac{R}{\tan \left(y-a_{2}\right)}-\frac{a_{1}}{\sin \left(y-a_{2}\right)}\right]^{T}\right\}
$$

but the kernel is only defined when the actuator is deflected from its equilibrium pose. In order to change the stiffness preset, the spring still needs to be tensioned or relaxed.

The maps $A(a, y): T_{a} \mathcal{A} \rightarrow T_{q} \mathcal{Q}$ of the other VSA designs presented in Section 2.2.3 do not have a kernel, and thus these VSAs cannot change their stiffness energy efficiently. By changing their internal configuration, they load their internal springs, meaning less energy can be absorbed from the environment.

\subsubsection{Energy Storage Capacity}

VSAs distinguish themselves from conventional actuators through their ability to store mechanical energy in internal elastic elements. The amount of energy that can be stored depends on the capacity of the internal springs, which cannot be extended past their elastic limits, and on the mechanism used to adjust the stiffness, and in most cases also on the actuator's stiffness setting. Generally, the spring state $q$ depends on both the output and the actuator configurations $y$ and $a$, and is affected by changing either of these variables. This means that different VSAs may not be able to store the same amount of energy, even if they are equipped with the same springs. While the energy storage capacity of a VSA is an important factor in its performance, it is often not in the center of attention during the design phase, and it is difficult to compare the energy capacity of different VSAs due to differences in dimensions and operating principles. For this reason a measure to determine the actuator's energy capacity is introduced.

\section{Energy Absorption Measure}

The output port of a VSA, as shown in Figure 2.2, is characterized by its generalized position $y$ and force $\tau_{y}(q, y)$, which depends on the actuator configuration variables $a$ of the VSA. The generalized output displacement and force may either be rotational or linear, however, most VSAs are rotational actuators. The energy that is supplied to the VSA from the load is

$$
E(a, y)=\int_{y_{1}}^{y_{2}} \tau(a, y) \mathrm{d} y
$$

The output stiffness $K$ is defined in Equation 2.11 as the partial derivative of the output torque with respect to the position. If the actuator is not in a singular configuration, i.e. $K>0$, the equilibrium position $y^{*}$ of the output for which 


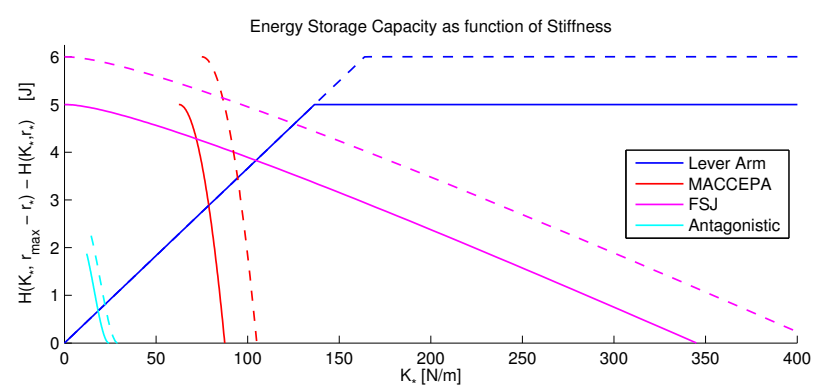

Figure 2.7: $M_{1}$, i.e. the energy storage capacity of VSAs based on a lever arm, MACCEPA, FSJ, and antagonistic mechansim. The solid lines show $M$ for $5 \mathrm{~J}$ internal storage capacity, while the dashed lines show internal springs with $6 \mathrm{~J}$ capacity to illustrate the effect of spring energy capacity on VSA performance.

$\tau_{y}\left(a, y^{*}\right)=0$ is uniquely defined by $a$. This means that there also exists a unique stiffness $K\left(a, y^{*}\right)=K^{*}(a)$ at the equilibrium position $y^{*}(a)$.

The output is coupled to the internal springs such that an output displacement $\Delta y$ results in an elongation $q$ of the internal springs. The energy $H(q)$ stored in the springs is in turn given by the spring displacement. For each spring, $q_{i}$ must remain in the set of permissible spring states $q_{i} \in \mathcal{Q}:=\left\{q \mid q_{\min } \leq q \leq q_{\max }\right\}$, which limits the energy that can be stored in a particular VSA. For a given configuration $\bar{a}$ the map $Q \bar{a}(y):=q(\bar{a}, y)$ can be defined, and the largest permissible output deflection $y_{\max }$ defined as the pre-image of $q_{\max }: y_{\max }:=Q \bar{a}^{-1}\left(q_{\max }\right)$.

$M$ is an absolute measure of the maximum amount of energy that the actuator's springs can absorb from the environment at a given stiffness setting.

$$
\begin{aligned}
M(a) & :=E_{\max }\left(K_{*}\right)=\int_{y_{*}}^{y_{\max }} \tau_{y}\left(K_{*}, y\right) \mathrm{d} y \\
& =H\left(K_{*}, y_{\max }\right)-H\left(K_{*}, y_{*}\right)
\end{aligned}
$$

\section{Evaluation of the Measure}

Figure 2.7 shows the measure $M$ for the different VSA designs as a function of their stiffness preset. In order to obtain comparable results, the actuators are designed with the same mechanical parameters. Namely, the energy capacity of their internal springs $H_{\max }=5 \mathrm{~J}$, maximum spring elongation $q_{\max }=0.02 \mathrm{~m}$, maximum output deflection $r_{\max }=\pi / 8 \mathrm{rad}$ and $R=0.05 \mathrm{~m}$, with the exception of the antagonistic design 2.2 .3 where $R$ is chosen as $\frac{q_{\max }}{2 y_{\max }}=0.025 \mathrm{~m}$ to achieve maximum spring deflection at maximum output deflection, as well as two separate springs with an energy capacity of $2.5 \mathrm{~J}$ each.

The lever arm mechanism shows a linear relation between energy storage capacity and stiffness until it saturates at the capacity of the internal spring and theoretically retains this value until infinity. This is because at low stiffnesses the spring elongation 
is limited by the output deflection, and the stiffness characteristic is not progressive as with the FSJ or antagonistic designs. Analogously, the spring state at higher stiffnesses is limited by the output torque that the actuator can withstand: the theoretical full availability of the energy storage capacity at infinite stiffness would require an infinite torque. It should be noted that although this design can be realized with a single tension spring, most actuators implement it with two antagonistically pretensioned springs, which reduces the percentage of the internal springs' energy capcity available at the output.

The MACCEPA mechanism shows a relatively limited stiffness range, over which the energy absorption capacity diminishes. However, this performance is still sufficient for its intended application in robotic limbs, where the design's capability for a large deflection range is an advantage, and the springs can furthermore be placed along the limb, and thus be chosen larger.

The FSJ design shows a relatively large stiffness range with decreasing energy capacity. The stiffness, however, is progressive and strongly coupled to the output deflection, which is also the reason why $M$ reaches its highest value at zero stiffness at the equilibrium position.

The antagonistic design shows the worst utilization of its internal springs, both in terms of storage capacity and stiffness range. Even in its most compliant setting, less than half of the energy storage capacity of the springs is available at the output due to pretensioning: when one spring is elongated through an output deflection, the other is relaxed, thus releasing stored energy.

Out of the presented designs, only the lever-arm based VSA is able to change its equilibrium stiffness without pretensioning its internal springs. The effect on its energy storage capacity becomes clear in Figure 2.7, where the proposed measure $M$ remains at its maximum value at higher stiffness settings.

\subsection{Design for a Lever Arm Based VSA}

The previous Section showed that VSA based on a variable transmission, such as variable lever-arm ratios, are well suited as actuators with a large energy absorption, as they preserve their internal storage capacity even as they increase their output stiffness. This section presents the working principle of a lever-arm-based VSA in more detail, and derives design requirements from an analysis of its internal loads and the way the VSA stores energy in its internal springs.

\subsubsection{Kinematic Model}

Figure 2.8 shows a lever-arm based stiffness adjustment mechanism with movable pivot to change the actuator's output stiffness. A difference to the model presented in Section 2.2.3 is the longer lever, which extends beyond the hub of the crank, and 


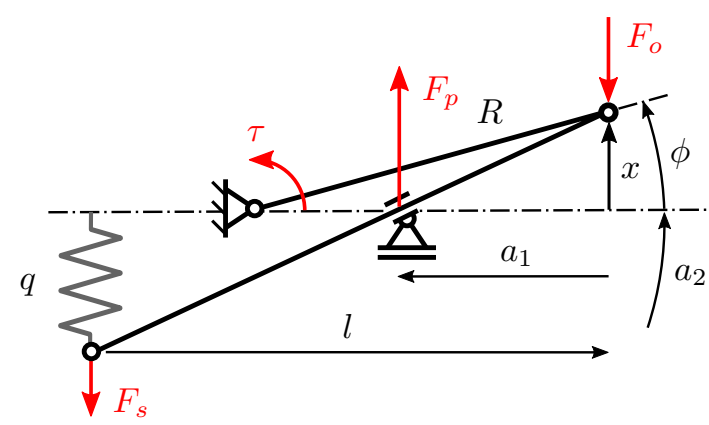

Figure 2.8: The modified lever arm design, in which the length of the lever $l$ is greater than the radius $R$ of the output crank. The torque $\tau$ at that crank exerts the force $F_{o}$ at the levers connection with the output, causing the reaction forces $F_{p}$ and $F_{s}$ at the pivot point and spring. The angular and linear deflections $\phi$ and $x$ at the output end of the lever cause the spring elongation $q$, depending on the pivot position $a_{1}$.

exploits the free volume of the actuator better. The equations presented in this section use approximations for small output deflections to allow the analytic derivation of representative equations, but still offer a good representation of the mechanism's properties and are a useful tool for analysis.

With reference to Figure 2.8, a crank with length $R$ transforms the linear force $F_{o}$ and deflection $x$ of the lever into the rotational torque $\tau$ and deflection $\phi$. A deflection $x=\sin (\phi) R$ of the lever at the connection with the output causes an elongation $q$ of the spring:

$$
q=\frac{l-a_{1}}{a_{1}} x=\frac{l-a_{1}}{a_{1}} \sin (\phi) R
$$

where $l$ is the length of the lever, and $a_{1}$ the position of the pivot point of the lever. Note that if more than one spring is connected to the lever, usually in an antagonistic setup, they can be considered as a single virtual spring.

The internal forces on the lever at the connection points with the springs, output, and pivot are

$$
\begin{aligned}
& F_{s}=k_{s} \cdot q, \quad F_{o}=\frac{l-a_{1}}{a_{1}} \cdot F_{s} \\
& F_{p}=F_{s}+F_{o}=\frac{l}{a_{1}} \cdot F_{s}
\end{aligned}
$$

where $k_{s}$ is the elastic constant of the internal springs. It follows that the output torque $\tau=F_{o} \cdot R$ is

$$
\tau=\frac{l-a_{1}}{a_{1}} \cdot R \cdot k_{s} \cdot q=\left(\frac{l-a_{1}}{a_{1}} \cdot R\right)^{2} \cdot k_{s}
$$



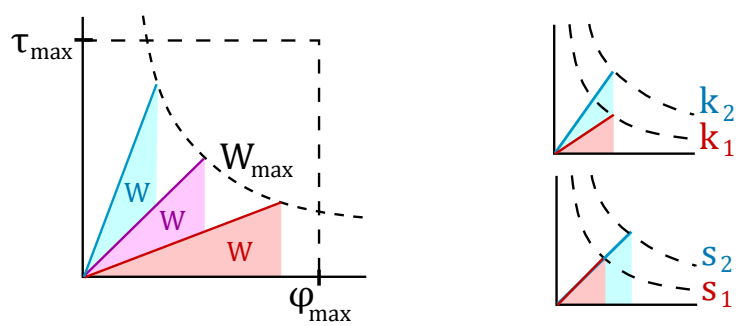

Figure 2.9: The torque-deflection workspace of a lever-arm based VSA is limited by the maximum torque $\tau_{\max }$, deflection $\phi_{\max }$ and energy capacity of the internal springs $W_{\max }$, which is the area under the torquedeflection characteristic. $W_{\max }$ depends on the stiffness $k$ and deflection $q$ of the spring, as the energy stored in a linear spring, used as example here, is $\frac{1}{2} k q^{2}$. Increasing the stiffness $k$, linearly increases the output torque for a given deflection and stiffness setting, while increasing $q$ allows the VSA to be deflected further, scaling the absorbed energy with $q^{2}$, as can be seen in the right part of the Figure.

and the output stiffness of the SAM (Stiffness Adjustment Mechanism)

$$
K=\frac{\partial \tau}{\partial \phi}=\left(\frac{l-a_{1}}{a_{1}} \cdot R\right)^{2} \cdot k_{s}
$$

which can indeed be adjusted by varying $a_{1}$, and scales linearly with $k_{s}$.

\subsubsection{Torque-Deflection Workspace}

A sufficiently large torque-deflection workspace is of central importance to the performance of a VSA. The actuator's ability to change its output stiffness, can only be exploited, if the VSA can exchange energy with the environment. For instance during a collision, the VSA still needs to absorb and store the kinetic energy of the impact by deflecting under the load, even when set to a different stiffness. The total work the actuator needs to absorb remains the same. The maximum energy that can be absorbed through the output, is the energy that the VSA can store in its internal spring element:

$$
W_{\max }=\int_{0}^{\phi_{\max }} \tau(\phi) \mathrm{d} \phi=\frac{1}{2} k_{s} q_{\max }^{2}
$$

with the output torque $\tau$ and deflection $\phi$, and spring deflection $q$ and stiffness $k_{s}$ for the case of a linear spring.

It is clear from Equation 2.26 that the torque-deflection workspace can be increased by stiffer springs or a larger maximum spring deflection, i.e. a larger energy storage of the springs.

The workspace boundary can be expressed through pairs of $\tau$ and $\phi$ at the maximum spring deflection. The output deflection $\phi(a, q)$ as a function of the spring 
deflection $q$ is obtained by reformulating Equation 2.21:

$$
\phi=\arcsin \left(\frac{a_{1}}{l-a_{1}} \cdot \frac{q}{R}\right)
$$

while the output torque $\tau(a, q)$ is given in Equation 2.24.

The connection between energy storage in the VSA and the spring parameters $k_{s}$ and $q_{\max }$ is illustrated in Figure 2.9, together with the influence of $k_{s}$ and $q_{\max }$ on the VSA's torque-deflection workspace.

\subsubsection{Internal Loads}

It can be seen from Equations 2.22 and 2.23, that the internal loads on the pivot point of the variable stiffness mechanism do not only depend on the output force $F_{o}$ and spring force $F_{s}$, but also on the transmission ratio between output and spring, defined by the pivot position $a_{1}$. The same output load might lead to undesirably large internal forces at unfavorable lever-arm ratios.

Equation 2.27 can be rewritten to give the pivot position $a_{1}$ for a given output and spring deflection

$$
a_{1}=\frac{l}{1+\frac{q}{R \cdot \sin (\phi)}}
$$

The ratio of the pivot and output forces is

$$
\frac{F_{p}}{F_{o}}=\frac{l}{l-a_{1}}=1+\frac{R \cdot \sin (\phi)}{q}
$$

The higher this ratio is, the larger the internal forces acting in the mechanism become for a given load at the output.

Figure 2.10 shows $\frac{F_{p}}{F_{o}}$ as a function of the maximum spring deflection $q_{\max }$ for several output deflections. It can be seen that the load on the pivot point becomes much larger compared to the force at the output, at small spring deflections. This is because a small spring deflection requires the pivot to be close to the springs, if a large output deflection is required, which leads to unfavorable lever arm ratios with respect to the internal loads.

\subsubsection{Design Guidelines}

The key design requirements for the springs for a lever arm based VSA have been identified to be:

- Energy Storage Capacity: Maximize the energy storage capacity of the springs to allow a large torque-deflection workspace and compliant interaction with the environment. 


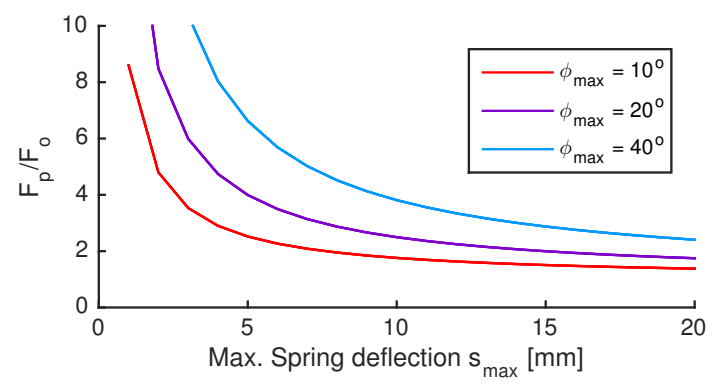

Figure 2.10: The ratio of the forces at the pivot point and the output at maximal deflection and load as function of the maximum spring deflection for $R=50 \mathrm{~mm}$.

- Internal Loads: Limit the internal loads on the pivot for a given output load.

- Compactness: The springs need to fit inside the typically cylindrical enclosure of the VSA, without obstructing the motion of the lever.

We address these requirements by proposing the following guidelines for the design of the elastic elements. These guidelines are elaborated in the remainder of the chapter.

- Energy Storage: The shape and material of the spring need to be chosen such that they maximize the energy storage capacity. A spring setup that avoids pretensioning the springs is highly preferable, as otherwise energy capacity and deflection range of the springs is lost to the pretensioning.

- Maximum Spring Deflection: Set a required maximum spring deflection $q_{\max }$, in this case $10 \mathrm{~mm}$, to limit the internal loads according to Equation 2.29. This means that the spring's stiffness $k$ needs to be maximized to achieve a maximum energy $E=\frac{1}{2} k q^{2}$, given the constraints on geometry, deflection, and stress.

- Utilize the Mounting Volume: To ensure a compact design, an $\Omega$-shaped leaf spring, shown in Figure 2.11, is chosen as elastic element through a heuristic approach. It exploits the available space better than the coil extension [91] [92] or torsion springs [93] that are mounted perpendicular to the lever in most other VSA! (VSA!) of this type, as elaborated in Section 2.5.2.

\subsection{Design Methodology for Elastic Elements}

The following section investigates how springs store elastic energy, and how leaf springs can be layed out to maximize their energy storage capacity, given the design requirements imposed by the stiffness adjustment mechanism. The equations 

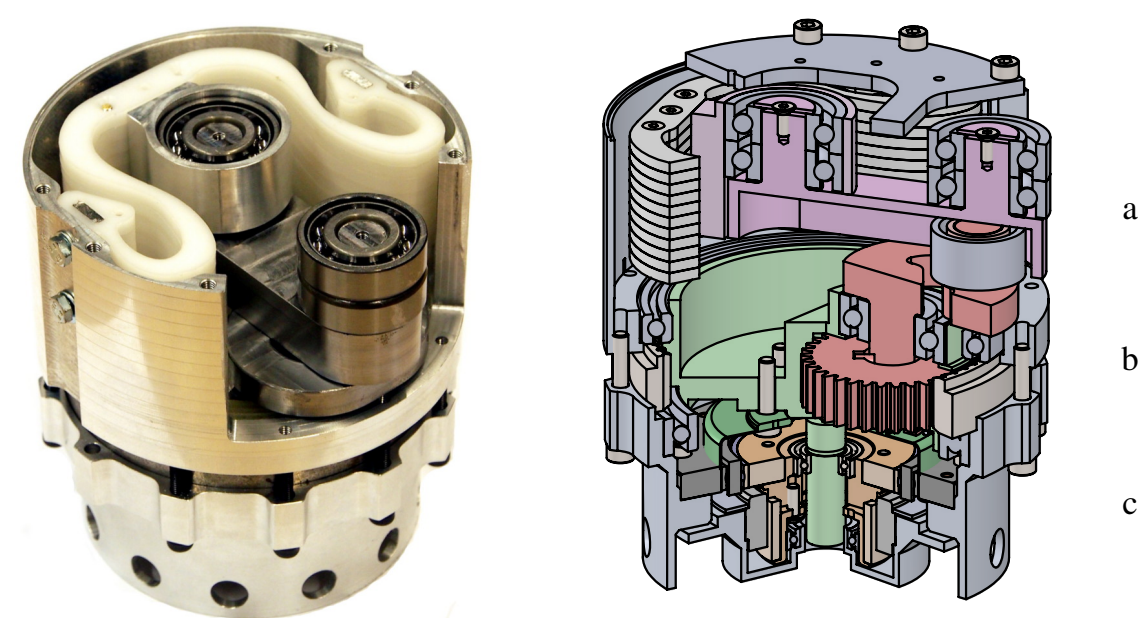

Figure 2.11: Lever-arm based stiffness adjustment mechanism equipped with an $\Omega$-shaped polymer (POM) leaf spring. The lever-arm mechanism a), planetary gear mechanism b), and actuation c) can be seen.

are derived for straight beams, but the design guidelines and relationships between the spring's parameters still hold true for the $\Omega$-shaped spring designed using a finite element analysis, as presented at the end of this section.

\subsubsection{Elastic Energy Storage}

Mechanical springs store potential energy as strain energy when they elastically deform under a load. A wide range of spring geometries has been developed over the centuries, including helical cylindrical tension and torsion springs, spiral torsion spring, or leaf springs. These different spring types are not only shaped differently, they also experience distinct types of strain and stress, such as normal, bending, shear, or torsional loads, as illustrated in Figure 2.12. The energy stored in a specimen of material with length $L$ and constant cross section area $A$ under a uniaxial tensional load $F$ is

$$
W=\int_{0}^{q} F(q) d q=\int_{0}^{q} k q d q=\frac{1}{2} \frac{E A}{L} q^{2}=\frac{\sigma^{2}}{2 E} L A=\frac{\sigma^{2}}{2 E} V
$$

with the stiffness $k=\frac{E A}{L}$ and the stress $\sigma=E \epsilon=E \frac{q}{L}$ induced in the material, which leads to the elongation $q=\frac{\sigma L}{E}$, where $E$ is the Young's modulus, $\epsilon$ the strain, and $V$ the volume of the specimen. When $\sigma$ is equal to the maximum permissible stress, this represents the maximum amount of energy a material can absorb under ideal conditions, before it fails. The specific energy capacity of a given volume of 
material under homogeneous tensile load is thus

$$
\frac{W_{i d e a l}}{V}=\frac{\sigma_{\max }^{2}}{2 E}
$$

In order to reach this value, the entire volume of material needs to be loaded. In many load cases, however, this is not possible, as localized stress peaks appear, and parts of the material are not (fully) utilized for energy storage. For a solid cylindrical rod of length $L$ and radius $R$ under a torsional load $T$ the energy stored in the rod is

$$
W=\int_{0}^{\phi} T(\phi) d \phi=\int_{0}^{\phi} k \phi d \phi=\frac{1}{2} \frac{G I_{p}}{L} \phi^{2}=\frac{1}{2} \frac{T^{2} L}{G I_{p}}
$$

with the torsional stiffness $k=\frac{G I_{p}}{L}$ and rotational deflection $\phi=\frac{T L}{G I_{p}}$ (analogous to the elongation $q=\frac{F L}{E A}$ ), with the shear modulus $G$ and the polar moment of inertia $I_{p}=\int r^{2} d A=\frac{\pi}{2} R^{4}$. As the torsional stresses are not distributed equally along the radius, the maximum stress $\tau_{\max }=\frac{T R}{I_{p}}$ occurring at the rod's surface needs to be taken to obtain the torque as $T=\frac{\tau_{\max } I_{p}}{R}$, and the energy stored in the rod as

$$
W=\frac{1}{2} \frac{T^{2} L}{G I_{p}}=\frac{1}{2} \frac{\tau_{\max }^{2} L I_{p}}{G R^{2}}=\frac{1}{2} \frac{\tau_{\max }}{2 G} \pi R^{2} L=\frac{1}{2} \frac{\tau_{\max }}{2 G} V
$$

Two differences between Equations 2.30 and 2.33 appear. Firstly the different stresses $\sigma$ and $\tau$, and elastic moduli $E$ and $G$, owed to the different material properties for the load cases of tension and shear, and secondly an additional factor $1 / 2$ in Equation 2.33. The ideal specific energy capacity for a material loaded under shear stress can be defined analogous to Equation 2.31 as

$$
\frac{W_{i d e a l}}{V}=\frac{\tau_{\max }^{2}}{2 G}
$$

That this value, however, is not reached for the torsion bar, due to the additional factor of $1 / 2$. This factor is the degree of volume utilization

$$
\eta_{A}=\frac{W}{W_{\text {ideal }}}
$$

which expresses the extent to which the spring material is being used for energy storage for a specific load condition and geometry. The torsion rod has a lower volume utilization, as the material closer to the center experiences smaller stresses, and thus absorbs less energy with respect to its maximum theoretical value. This is also the reason that for instance torsionally loaded drive shafts are often executed as hollow shafts. Figure 2.12 illustrates the stress distribution in materials for basic load cases.

It is possible for $\eta_{A}$ to reach values larger than one for some spring types, like annular or cup springs. However, this additional energy is dissipated through friction, and not stored. 


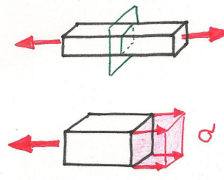

(a) Tension

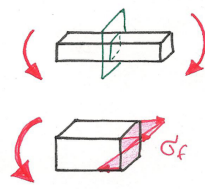

(b) Bending

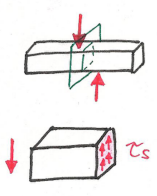

(c) Shear

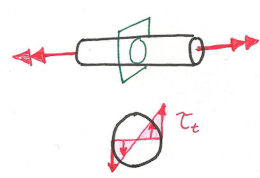

(d) Torsion

Figure 2.12: Basic load cases and the resulting stress distributions in the intersected members. Note that bending results in the same kind of load as normal forces for an isotropic material. However, the material's reaction can differ when the material properties are not symmetric in tension and compression, and due to statistical effects that determine material failure due to different stress distributions between bending and tension.

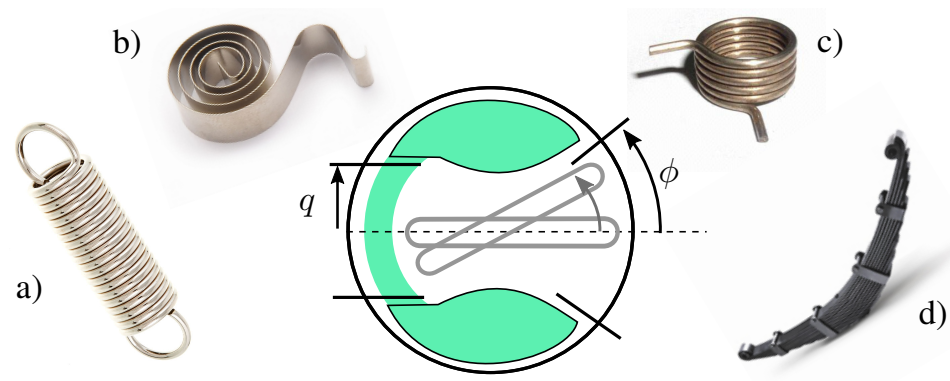

Figure 2.13: The mounting volume (green) available for the elastic elements inside the round variable stiffness mechanism depending on the output deflection $\phi$ and spring deflection $q$, along with several spring types: a) helical tension, b) spiral torsional, c) helical torsional, and d) leaf spring.

The spring type and geometry, together with the specific energy capacity of the material, determine how much energy can be stored in a given volume of spring material. Other important factors, such as the spring's stiffness, maximum deflection, or mounting volume will be addressed below.

\subsubsection{Mounting Volume and Spring Type}

The mounting volume for the internal springs highly depends on the application and requirements. For instance in cases where it is possible to place the springs along the links of the robot this can be a good solution to accommodate long and narrow tension springs. In the present case, the aim was to integrate the springs within the cylindrical enclosure of the variable stiffness mechanism. The springs thus need to fit inside the free area that is not taken up by lever arm mechanism, and allow the required deflection, as shown in Figure 2.13.

Many lever arm based VSAs use pretensioned helical tension or compression springs [91, 101, 92], or torsional springs that act like virtual linear springs by us- 

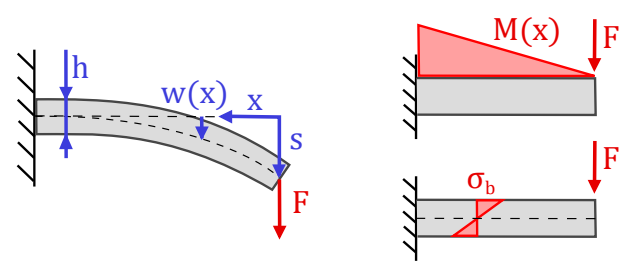

Figure 2.14: A cantilever bending beam with one fixed and one free end loaded by the force F, and the distribution of the resulting bending moment and stress.

ing their legs as a lever [93]. However, these solutions leave much of the available space unused. The choice of the spring should however optimally exploit the mounting volume, and maximize the spring's volume for energy storage, while still allowing enough deflection and fulfilling the design requirements presented in Section 2.4.4. Figure 2.11 shows the $\Omega$-shaped leaf spring that is chosen as elastic element for this application, which neatly fills the mounting volume, while allowing for good spring performance by maximizing the active length of the leaf spring. What is more, is that the leaf spring can be loaded in both directions, i.e. in tension and compression. Most other lever arm based VSAs use antagonistically pretensioned springs, that remain either in tension, or compression at all times, which may be easier to realize from a constructional point of view, but undesirable when considering that half the spring's available deflection is lost for pretensioning.

\subsubsection{Euler-Bernoulli Beam}

In order to investigate their elastic properties, leaf springs can be modeled as an EulerBernoulli beam. This is a simplification of the general Timoshenko beam theory which neglects shear in the beam. As this analysis aims to produce qualitative guidelines for the parameters determining the springs performance, and not a quantitative prediction of the loads or displacements, a slender beam with small deflections $w(x)$ and boundary conditions as shown in Figure 2.14 can be assumed, where $x$ denotes the distance from the spring's free end along its neutral fiber. For this simple case of a beam with constant, rectangular cross section with height $b$ and thickness $h$, area moment of inertia $I=\frac{b h^{3}}{12}$, and constant flexural modulus $E$, with one fixed end, and that is loaded with the force $F$ at its free end the maximal deflection occurs at the free end of the lever and is [119]

$$
w(0):=q=\frac{F \cdot l^{3}}{3 \cdot E \cdot I}=\frac{4 \cdot F \cdot l^{3}}{E \cdot b \cdot h^{3}}
$$

The stiffness of the spring is

$$
k=\frac{d F}{d q}=\frac{3 E I}{l^{3}}=\frac{E b h^{3}}{4 l^{3}}
$$


The maximum energy that can be stored in the spring now depends on the maximum spring deflection, achieved when the stress in the spring reaches its elastic limit. The maximum bending stress occurs at the surface of the fixed end of the beam, where the moment $M_{b}(l)$ is largest [119]

$$
\sigma_{\max }=\frac{M_{b}(l)}{I} \frac{h}{2}=\frac{6 F l}{b h^{2}}
$$

leading to the load

$$
F=\frac{\sigma_{\max } b h^{2}}{6 l}
$$

which, substituted into Equation 2.36, gives the maximum deflection

$$
q_{\max }=\frac{2}{3} \frac{l^{2}}{h} \frac{\sigma_{\max }}{E}
$$

And thus the maximum energy that can be stored in the spring is

$$
\begin{aligned}
W_{b \max } & =\frac{1}{2} F q_{\max }=\frac{1}{2} k q_{\max }^{2}=\frac{1}{2} \frac{E b h^{3}}{4 l^{3}}\left(\frac{2}{3} \frac{l^{2}}{h} \frac{\sigma_{\max }}{E}\right)^{2} \\
& =\frac{1}{9} \frac{\sigma_{b}^{2}}{2 E_{b}} b h l=\eta_{A} \frac{\sigma_{b}^{2}}{2 E_{b}} V
\end{aligned}
$$

with the degree of volume utilization $\eta_{A}=1 / 9$, specific energy absorption capacity $\frac{\sigma_{b}^{2}}{2 E_{b}}$ and volume $V=b h l$.

The degree of volume utilization $\eta_{A}$ [120], or form coefficient $C_{F}$ [119], accounts for a non-uniform stress distribution, comparing the actual energy stored in the material with the highest possible energy stored in the same volume.

\subsubsection{Optimizing Spring Parameters}

Equation 2.41 shows that the maximum energy capacity of a spring depends on three factors:

- The degree of volume utilization $\eta_{A}$,

- The specific energy storage capacity $\frac{\sigma_{\max }^{2}}{2 E}$ of a material,

- The total volume $V$ of the spring.

Equation 2.41 also shows, that a leaf spring with a constant cross section does not utilize its material very well for energy storage. This is because the bending moment is not constant along its length, as shown in Figure 2.14, and the highest stress only occurs at the spring's fixed end, leading to the stress distribution

$$
\sigma_{\max }(x)=\frac{M(x)}{I(x)} \frac{h}{2}=\frac{6 F x}{b(x) h(x)^{2}}
$$


For a parabolic spring with variable thickness $h(x)=h_{0} \sqrt{x / l}$, or a triangular spring with variable height $b=b_{0} x$, however, the maximum stress is spread evenly over the lateral surface of the spring. The degree of volume utilization for such a spring of uniform strength is $\eta_{A}=\frac{1}{3}$ [120], three times larger than for a constant cross section. The boundary conditions also influence the way the spring is loaded, and thus the stiffness and deflection range, as demonstrated by [95]. The energy storage capacity of a leaf spring can thus be much improved by choosing a favorable geometry, leaving the dependency on the material properties and spring volume to be investigated.

Usually the mounting dimensions only constrain the height $b$ and length $l$, while the thickness $h$ is often not significant for the volume of the variable stiffness mechanism. This is because leaf springs are slender structures, and their thickness is typically much smaller than their length.

For a given deflection $q_{\max }$, height $b$, and length $l$, the thickness $h$ of the beam can be chosen, such that the bending stress $\sigma$ does not exceed the permissible stress $\sigma_{\max }$, which gives the thickness of the beam from Equation 2.40 as

$$
h=\frac{2 l^{2} \sigma_{\max }}{3 E q_{\max }}
$$

This represents a way to maximize the spring's volume and thus energy absorption, given constraints on $l, b$ and $q$. The spring's volume $V=l \cdot b \cdot h$ is now also a function of its maximum deflection $q_{\max }$ and the material properties $\sigma_{\max }$ and $E$. Substituting Equation 2.43 into Equation 2.41, the maximum energy the spring can absorb becomes

$$
W_{b}=\frac{1}{9} \frac{\sigma_{\max }^{2}}{2 E} b h l=\frac{1}{27} \frac{b l^{3}}{q_{\max }} \frac{\sigma_{\max }^{3}}{E^{2}}
$$

and substituting Equation 2.43 into Equation 2.37 yields its stiffness as

$$
k=\frac{2}{27} \frac{b l^{3}}{q_{\max }^{3}} \frac{\sigma_{\max }^{3}}{E^{2}}
$$

This equation illustrates the trade-offs encountered when stiffening the spring for increasing the energy capacity, given geometric and material constraints. The maximum deflection $q_{\max } \sim \sqrt[3]{k^{-1}}$ decreases, while the peak stress $\sigma_{\max } \sim \sqrt[3]{k}$ increases, as shown in Figure 2.15, for a thicker stiffer spring.

Note that $\frac{\sigma_{\max }^{3}}{E^{2}}$ appears as a new material index, that is proportional to the spring's stiffness and energy absorption capacity, and is determined by the material properties given in Table 2.1. The material properties are thus not only important for the specific energy storage capacity, but also for the additional constraint on a required spring deflection $q_{\max }$, and the index $\frac{\sigma_{\max }^{3}}{E^{2}}$ becomes a guide for material evaluation and selection. 


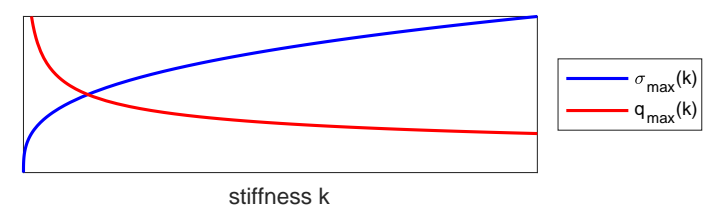

Figure 2.15: The influence of the stiffness of bending beam, given geometric and material constraints: A stiffer beam has a smaller deflection $q_{\max } \sim \sqrt[3]{k^{-1}}$ but higher peak stress $\sigma_{\max } \sim \sqrt[3]{k}$.

Table 2.1: Material Properties for Selected Spring Materials under Normal, Flexural, and Torsional Loads

\begin{tabular}{|c|c|c|c|c|}
\hline Material & $\begin{array}{c}\text { Elastic Modulus }^{1} \\
{[\mathrm{GPa}]}\end{array}$ & $\begin{array}{c}\text { Yield Strength }^{2} \\
{[\mathrm{MPa}]}\end{array}$ & $\begin{array}{l}\text { Energy Capacity } \\
\frac{\sigma^{2}}{2 E}, \frac{\tau^{2}}{2 G}[\mathrm{MPa}]\end{array}$ & $\begin{array}{c}\text { Flexion Index }^{3} \\
\sigma_{f}^{3} / E_{f}^{2}[\mathrm{kPa}]\end{array}$ \\
\hline \multirow{2}{*}{ Spring Steel (51CrV4) [121] } & $E=206$ & $\sigma=840 \ldots 1260$ & $1.71 \ldots 3.85$ & \multirow{2}{*}{$14.0 \ldots 47.1$} \\
\hline & $G=78$ & $\tau=600 \ldots 900$ & $2.30 \ldots 5.19$ & \\
\hline \multirow{2}{*}{ Beryllium Copper (CuBe2) [121] } & $E=120$ & $\sigma=665 \ldots 805$ & $1.84 \ldots 2.71$ & \multirow{2}{*}{$20.4 \ldots 36.2$} \\
\hline & $G=47$ & $\tau=475 \ldots 575$ & $2.40 \ldots 3.52$ & \\
\hline \multirow{2}{*}{ Phosphor Bronze (CuSn6) [121] } & $E=115$ & $\sigma=630 \ldots 825$ & $1.72 \ldots 2.96$ & \multirow{2}{*}{$18.9 \ldots 42.5$} \\
\hline & $G=42$ & $\tau=450 \ldots 590$ & $2.41 \ldots 4.14$ & \\
\hline \multirow{2}{*}{ Aluminum (ENAW-AlCu4SiMg) [121] } & $E=70$ & $\sigma=125 \ldots 380$ & $0.112 \ldots 1.03$ & \multirow{2}{*}{$0.399-11.2$} \\
\hline & $G=26$ & $\tau=90 \ldots 270$ & $0.156 \ldots 1.40$ & \\
\hline \multirow{2}{*}{ POM-C (Tecaform AH) [122] } & $E=2.8$ & $\sigma=67$ & 0.802 & \multirow{2}{*}{111} \\
\hline & $E_{f}=2.6$ & $\sigma_{f}=91$ & 1.59 & \\
\hline \multirow{2}{*}{ PET (Tecadur PET) [122] } & $E=3.3$ & $\sigma=91$ & 1.25 & \multirow{2}{*}{208} \\
\hline & $E_{f}=3.4$ & $\sigma_{f}=134$ & 2.64 & \\
\hline \multirow{2}{*}{ PEI (Tecapei) [122] } & $E=3.2$ & $\sigma=127$ & 2.5 & \multirow{2}{*}{405} \\
\hline & $E_{f}=3.3$ & $\sigma_{f}=164$ & 4.08 & \\
\hline
\end{tabular}

${ }^{1} E$ : Young's (tensile) modulus, $E_{f}$ : flexural modulus, $G$ : shear modulus

${ }^{2} \sigma$ : tensile yield strength, $\sigma_{f}$ : flexural yield strength, $\tau$ : shear yield strength

3 The material index $\sigma_{f}^{3} / E_{f}^{2}$ only applies to flexural loads because it was derived through geometric considerations for bending leaf springs.

\subsubsection{Spring Materials}

The previous analysis illustrates the importance that choosing a suited material by means of different material indices [123] has for a spring's performance. The specific energy capacity $\frac{W}{V}=\frac{\sigma^{2}}{2 E}$ is quadratic to the elastic limit of the material, and inversely proportional to its elastic modulus, meaning that tough, i.e. strong and elastic, materials are required. As such, high strength spring steels, copper and nickel alloys are common spring materials $[119,121,124]$, but also plastics, mostly fibre-reinforced polymers, and rubbers are being used for special applications [124]. Being light and easy to shape, engineering plastic are finding new applications in robotics, especially in light of rapid prototyping techniques $[125,126]$ and the trend towards soft robotics and morphological computation [87, 127].

Table 2.1 lists the material properties of selected spring materials under normal $\sigma_{n}$, flexural $\sigma_{f}$, and shear loads $\tau$. For an ideal isometric material the flexural strength and modulus should be the same as those under a tensional load. This is, however, not always the case. The flexural strength and modulus can differ from those under tension, especially for engineering plastics, where the flexural strength is often higher. 


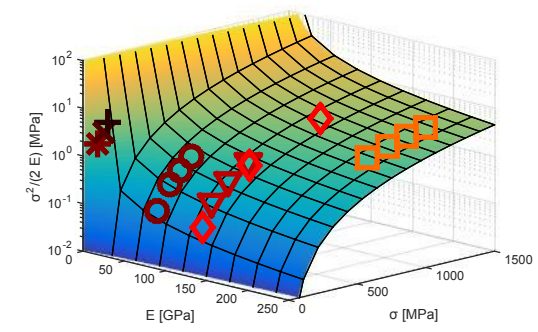

(a) The specific energy $\frac{\sigma^{2}}{2 E}$ of different materials.

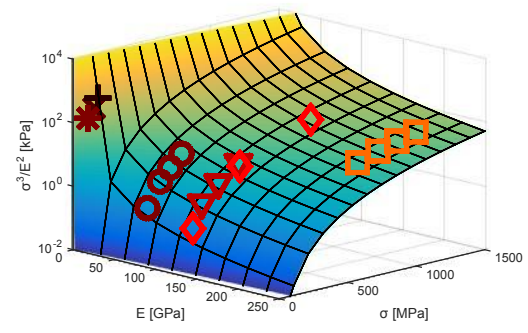

(b) The index $\frac{\sigma^{3}}{E^{2}}$ for different materials.

Figure 2.16: The specific energy $\frac{\sigma^{2}}{2 E}$ and the material index $\frac{\sigma^{3}}{E^{2}}$ according to Equation 2.44 for different spring materials. Plastics (POM: *, PET: X, PEI: +) with very low flexural modulus, aluminium (O), phosphor bronze $(\Delta)$ and beryllium copper $(\diamond)$ with intermediate rigidity, and steel $(\square)$ with high Young's modulus and strength. Due to their low flexural modulus, engineering plastics outperform strong but rigid metals in terms of $\frac{\sigma^{3}}{E^{2}}$. Note the logarithmic scale of both figures.

This may be caused by asymmetries of the material properties under tension or compression, or by statistical effects of failure modes in different load conditions.

The selected values show the wide range of different material properties and their effect on the energy storage capacity of the material. To illustrate this further, Figures 2.16a and 2.16b show the specific energy capacity $\frac{\sigma_{\max }^{2}}{2 E}$, and the index $\frac{\sigma_{\max }^{3}}{E^{2}}$ of the materials as a function of their flexural yield strength and modulus. It can be seen that even though metals generally have a higher specific energy capacity $\frac{\sigma_{\max }^{2}}{2 E}$ than plastics, engineering plastics can achieve a higher energy storage when considering $\frac{\sigma_{\max }^{3}}{E^{2}}$, which takes the special requirements of lever arm VSAs into account.

One should note the range of different values, especially the yield strength, for each material. It namely depends on many factors, such as the chemical composition, production process, or heat treatment. Likewise the number of materials presented here is far from exhaustive, but does show representative samples of the most common materials used for springs over a large range of strength and elasticity.

Two materials with vastly different properties are evaluated in the following analysis. POM (Polyoxymethylene), also known as acetal or Delrin, is a widely available and easy to process engineering plastic, with excellent fatigue strength and creep resistance [124]. With a flexural modulus of only $2.6 \mathrm{GPa}$ and a yield strength of $91 \mathrm{MPa}$ it represents the compliant polymers. As rigid but strong metal, $75 \mathrm{Cr} 1+\mathrm{QT}$ is a tool steel that is used for a variety of spring and tool applications, with similar properties as the popular spring steel $\mathrm{C} 75 \mathrm{~S}$, but better availability in plates (which were used for laser cutting and stacking the layers of the spring, like with POM). Hardened and tempered, it has a Young's modulus of $210 \mathrm{GPa}$ and yield strength of $950 \mathrm{MPa}$. 


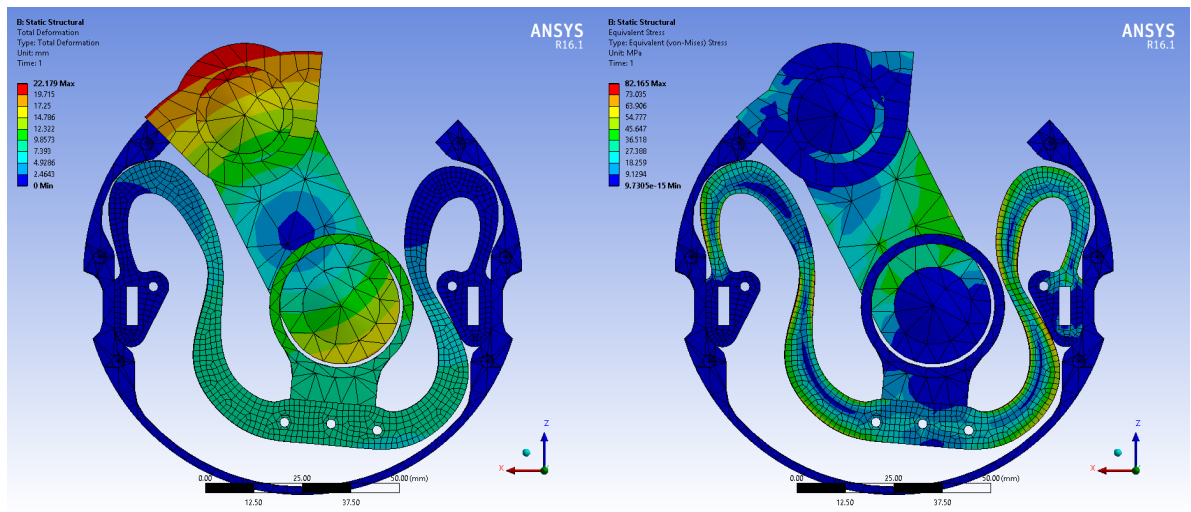

Figure 2.17: Results of the FEA showing the total deflection and von-Mises stress for the POM spring at full deflection.

\subsubsection{Finite Element Analysis}

The design guidelines derived in this section were put into effect with a FEA. The springs need to fit within the mounting volume, without obstructing the lever, and were laid out for a maximum deflection $s_{\max }=10 \mathrm{~mm}$, as motivated in Section 2.4.3. In order to maximize the energy capacity, the springs were designed to be as stiff as possible by achieving an as uniform as possible stress distribution close to the permissible stress. This has been carried out in Ansys for two materials, spring steel and POM, with flexural moduli and yield strengths of $210 \mathrm{GPa}$ and $950 \mathrm{MPa}$ for steel, and 2.6 GPa and $90 \mathrm{MPa}$ for POM respectively.

The stress in the spring was verified for a range of pivot positions of the lever, as the precise stress distribution and motion of the spring depend on the internal transmission. Figure 2.17 shows the deformation and stress of the POM spring at maximum spring and output deflection. Large deformation effects were taken into account to deal with the geometric nonlinearities caused by changes of the spring's shape, requiring an iterative solution for the FEA.

Figure 2.19 shows the simulated torque-deflection workspace of the springs. It can be seen that the POM spring achieves a larger workspace than the steel spring; the more flexible POM could be made much thicker and thus stiffer than steel, while still reaching the same maximum deflection, as predicted by the index $\frac{\sigma^{3}}{E^{2}}$.

\subsection{Experiments}

In order to verify the results of the FEA, the lever arm based variable stiffness mechanism, shown in Figure 2.11, was equipped with both a spring made from spring steel and POM, that were manufactured from stacked layers that were laser-cut from $3 \mathrm{~mm}$ 


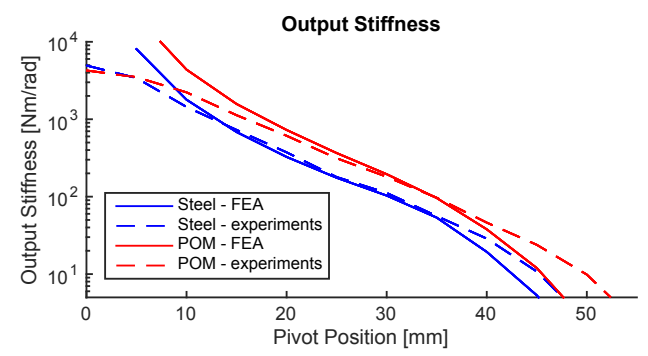

Figure 2.18: Semi-logarithmic graph of the simulated and measured output stiffness for a steel and POM spring as function of the pivot position.

thick sheet material. A section view showing the structure of the variable stiffness mechanism, that is based on the design of [91], is given in Figure 2.11. The lever-arm mechanism with leaf spring and pivot bearing can be seen at the top of the variable stiffness mechanism. Below it, a hypocyclic gear mechanism moves the pivot point in a straight line. The mechanism is actuated through a RoboDrive motor and Harmonic Drive strain wave gear.

The torque-deflection characteristics, shown in Figure 2.20, were recorded by manually deflecting the output of the variable stiffness mechanism via a lever carrying a Schunk FT-Mini-40 force sensor, while measuring the output deflection with an integrated ams AS5047P absolute magnetic rotary encoder. The stiffness was adjusted between the measurements by changing the actuated pivot position of the variable stiffness mechanism in $5 \mathrm{~mm}$ increments along the length of the lever.

As predicted by the analysis and simulations in Section 2.5, the POM spring is stiffer and achieves a larger torque deflection workspace than the steel spring, though also shows more hysteresis, which is negligible for the steel spring. Both springs show an increased hysteresis in the third quadrant, which is likely caused by friction in the SAM setup. The play of ca. $2^{\circ}$ visible in the measurements is mostly caused by backlash in the actuation of the pivot.

The workspace limit, defined by the spring's end-stops, are shown in Figure 3.15 were calculated with the passive output deflection from Equation 2.27 at maximum spring deflection $s_{\max }=10 \mathrm{~mm}$ and the corresponding output torque from Equation 2.24. The spring stiffness has been set to match the experimental results, and was found to be approximately $0.085 \mathrm{~N} / \mathrm{mm}$ and $0.15 \mathrm{~N} / \mathrm{mm}$ for the steel and POM springs respectively.

The simulated and measured output stiffness is shown in the semi-logarithmic graph in Figure 2.18, and shows a good accordance between measurements and FEA simulations. Only for pivot positions below $15 \mathrm{~mm}$ the parasitic compliance of the variable stiffness mechanism becomes apparent, when the measured output stiffness for both springs approaches $5000 \mathrm{Nm} / \mathrm{rad}$ in stead of infinity. Likewise, for pivot positions above $35 \mathrm{~mm}$ the experimental stiffness values approach zero slower than in 


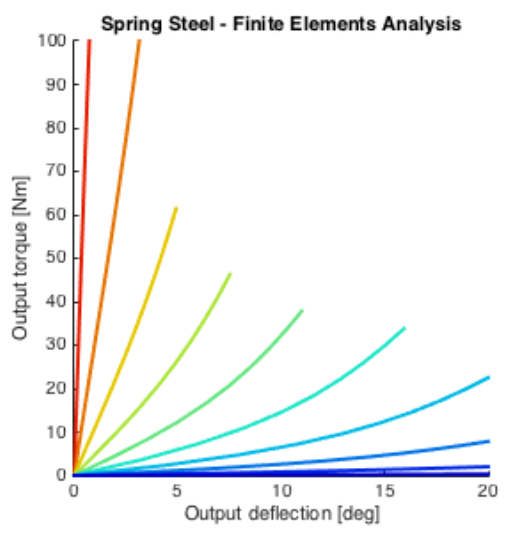

(a)

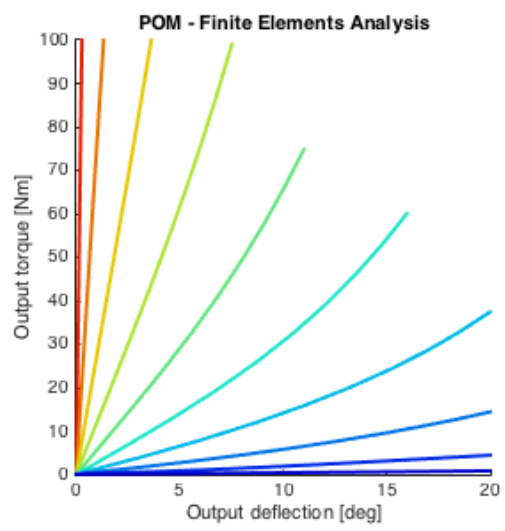

(b)

Figure 2.19: Simulated torque-deflection workspace from finite element analysis for a steel (Figure 2.19b) and POM spring (Figure 2.19a).

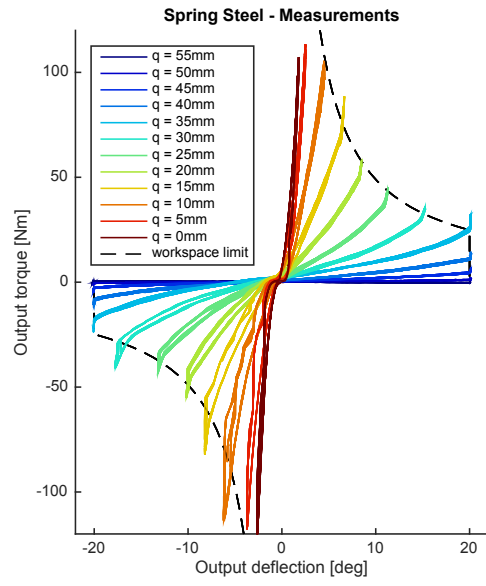

(a) Spring steel

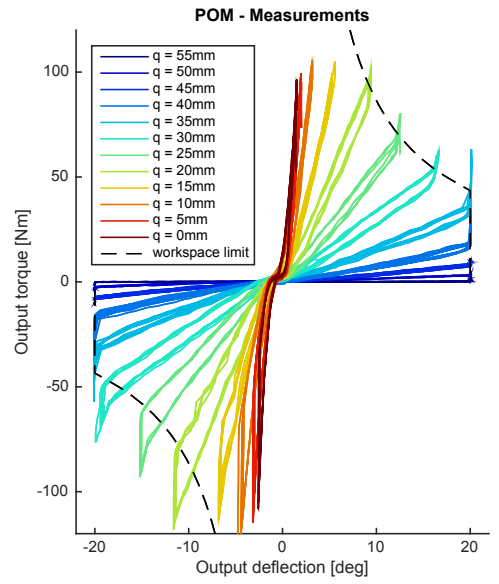

(b) POM

Figure 2.20: Torque-deflection measurements of the variable stiffness mechanism equipped with a POM and Steel spring for a range of different stiffness settings, determined by the pivot position. 
the simulations.

\subsection{Discussion}

Both the simulations and experiments confirm that a flexible polymer spring can achieve greater stiffness, and thus energy storage, than a strong metal spring, when they are laid out for the same spring deflection. This makes engineering plastics very suited as elastic elements in physically compliant robotic applications, which themselves are a growing field. Especially when considering the spread of rapid prototyping technologies, which drastically increases the ease of manufacturing of custom made polymer springs, while metal springs are available as off-the-shelf components.

Metal springs, on the other hand, are a proven material that exhibit other advantages over polymers, such as lower damping and hysteresis, higher resistance to creep, and a larger operating temperature range. However, when selecting a material for a spring element, its properties, manufacturing process, availability and cost all need to be taken into account. Engineering Polymers offer an interesting option, where other high performance polymers, or fiber-reinforced materials, can often even outperform POM, which was used in this comparison.

It has furthermore been shown here, that a suited method for designing an elastic element, can also drastically increase the springs performance, and a proper analysis is crucial for a high performance solution.

\subsection{Conclusions}

This chapter has addressed the dissertation's research objectives RO1 and RO2, which concern the modulation of the internal power flow of VSAs and how to maximize the energy capacity of their internal springs, by investigating in which way different types of VSAs utilize their internal elastic elements, and how these elements should be designed.

In order to meet RO1 the characteristics of different VSA mechanisms were analyzed through a port-based modeling approach, and a measure used to determine the energy a particular design can absorb in its internal springs. A lever arm based VSA was chosen, which adjusts its stiffness by varying the transmission between springs and output, and thus retains its energy absorption capacity over a wide stiffness range. After further analyzing the selected lever-arm based design and the present mounting conditions, a methodology and guidelines for the design of the internal springs were devised. This approach led to the use of custom $\Omega$-shaped polymer leaf springs, that provide a large internal energy storage of the actuator, while keeping the design compact.

The research objective $\mathbf{R O 2}$ was achieved through the design guides concerning the spring's shape, and the new material index, that evaluates the energy capacity for 
given geometric constraints.

It was shown that the theoretical analysis carried out, in combination with a FEA, can lead to a design that increases the VSA's torque-deflection workspace and energy storage capacity. Finally, the results were verified through experiments with a VSA equipped with steel and polymer springs.

With their energy efficient actuation, compact format, and large energy absorption capacity, the developed VSAs are well suited for robotic applications involving physical interaction with a dynamic environment. 



\section{CHAPTER 3}

\section{Mechatronic Design of a VARiable StifFness ROBOTIC ARM}

This chapter presents the mechatronic design of the robotic arm that is mounted on a ground rover and used to deploy and recover small-scale unmanned aerial vehicles in the context of the SHERPA mission. Design requirements are derived from the context of the rescue mission and a kinematic analysis is provided that leads to a customized design, including variable stiffness joints for compliant interaction with the environment.

The research objective $\mathbf{R O 3}$ is addressed by implementing an actuation topology that allows the desired range of workspace compliance with only 3 variable stiffness joints, limiting the overall system complexity, while still exploiting the benefits of passively compliant actuation. The engineering objective EO1 is met by determining the manipulator kinematics such that the arm can reach all required points in its workspace, and still be stowed in a compact volume for transport. The design, construction, and integration of the arm's components, as outlined in EO2, is also presented in the chapter. Experiments demonstrate the system's ability to perform the required Cartesian trajectory control and manipulation tasks, and to achieve a desired variable end-effector compliance.

This chapter has been previously published as:

E. Barrett, M. Reiling, G. Barbieri, M. Fumagalli, and R. Carloni. "Mechatronic design of a variable stiffness robotic arm." in Proceedings of the IEEE/RSJ International Conference on Intelligent Robots and Systems, 2017, pp. 4582-4588. 


\subsection{Introduction}

As already introduced in Chapter 1, the goal of the SHERPA project is a collaborative mission involving human rescuers and a heterogeneous team of autonomous ground and aerial robotic platforms for search and rescue scenarios [12]. Large- and smallscale UAVs (Unmanned Aerial Vehicles) gather data on the operational environment and localize victims, while a ground rover acts as a communications hub and base station for the small scale UAVs. In order to guarantee the autonomy of the UAVs over the whole course of the mission, the rover is equipped with a robotic arm capable of deploying and retrieving the UAVs to the rover, where their batteries are exchanged.

Complex applications, such as the tasks of the SHERPA arm, require robots to interact with unknown and unstructured environments, pushing the limits of dexterous and safe environmental interaction and human-robot cooperation.

Classical industrial robots are made to be mechanically rigid, which is beneficial for fast and precise position control tasks. An increasing number of robots, however, are implementing active impedance control, which allows them to better interact with the environment, execute dexterous tasks, and increase safety. Nonetheless, the robot's behavior and robustness is limited by the control bandwidth, which makes it impossible to absorb all arbitrary collisions without endangering the environment or people, or damaging itself.

The SHERPA arm is mounted on a rover that moves through rough terrains and needs to be extremely resilient against disturbances and shocks. The addition of mechanical springs not only protects the robot from impacts and shocks, but also the environment that it interacts with, making the robot intrinsically safe, by mechanically decoupling the robot's structure and actuators from rigid impacts.

SEAs (Series Elastic Actuators) [41] contain physical springs that exhibit a direct relation between the applied position and the force output, and react instantaneously to disturbances, and are capable of storing mechanical energy. However, their fixed compliance means that the robot's mechanical impedance and natural dynamics cannot be adapted physically to different tasks that require or benefit from these changes. VIAs (Variable Impedance Actuators) [42, 43], and in particular VSAs (Variable Stiffness Actuators), address this drawback by adjusting the mechanical stiffness of the robot's joints, and combine the safety, robustness, and increased dynamic performance of SEAs, with the versatility of active impedance. Robotic manipulators, such as the MIA Arm [78] and David (formerly the DLR Hand Arm System) [79], are pioneering the technology of VSAs and demonstrate their capabilities and broad potential applications.

This chapter presents the mechatronic design of the SHERPA arm, with a focus on the kinematic analysis of the workspace and the workspace compliance resulting from the chosen actuation topology, as well as on the mechanical design of the manipulator's components. The manipulator was built to retrieve and deploy UAVs for servicing, which requires dexterous interaction with an unknown environment, while 


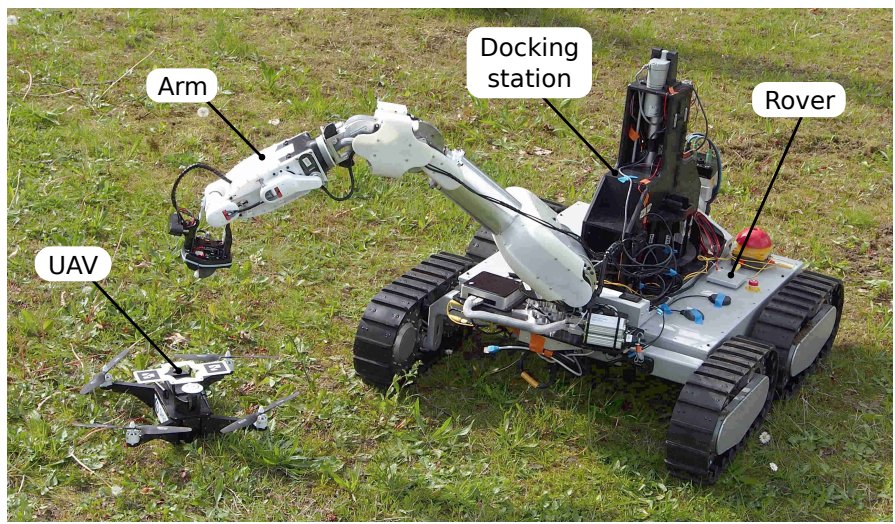

Figure 3.1: The SHERPA robotic arm mounted on the ground rover, together with the UAV and the docking station.

also keeping the mechanical system light and robust for mobile operations. A hybrid actuation topology with a combination of VSAs and classical stiff actuators strikes this balance of low overall system complexity and tunable mechanical workspace compliance. Figure 3.1 shows the developed arm mounted on the ground rover, together with the UAV and the docking station. The remainder of this chapter is organized as follows. Requirements for the arm are derived from the SHERPA mission in Sec. 3.2. The arm's workspace and achievable Cartesian compliance are analyzed in Section 3.3, its mechanical design is presented in Section 3.4, and the electronics and software architecture in Section 3.5 and 3.6. Experimental results validating the design are presented in Section 3.7, and conclusions are drawn in Section 3.8.

\subsection{Requirements}

This section specifies the arm's task within the SHERPA mission, which was detailed in Section 1.1, and puts the requirements on the performance of the manipulator outlined in Section 1.1.5 into more concrete terms.

\subsubsection{The SHERPA Mission}

The primary purpose of the robotic arm is to extend the UAV's operational time and range by docking them on the ground rover to enable the replacement of their batteries and deploying them. This is necessary, because the mission duration far exceeds the UAV's battery life, and a simple landing station was not deemed robust enough for the mission's adverse and remote environment. After the UAV has been localized and the ground rover moved into a position in which the UAV is in the arm's workspace, the docking sequence is initialized as a compound action, composed of the following 
elementary actions: i) reach: move the arm's end-effector into position above the $\mathrm{UAV}$; ii) grasp: engage and lock the interface with the gripper exploiting the arm's compliance and self-alignment; iii) dock: stow the UAV on the rover, using compliant manipulation to allow the battery to be exchanged; iv) deploy: place the UAV with replaced battery from the rover to the ground and release it, so it can continue its mission. While the reach and place actions represent motions of the arm in free space, with and without load, respectively, the grasp and dock actions explicitly require the arm to interact with and manipulate the environment.

\subsubsection{Design Requirements}

Derived from its role in the SHERPA mission, the main design requirements for the arm can be placed into the following categories:

1. Kinematics: The arm needs to reach all required positions, notably the docking position and a wide range of reach positions. While at least 6 -DOF are required to reach arbitrary positions in Cartesian space, a 7-DOF manipulator was chosen for better manipulability in an anthropomorphic configuration with a 3-DOF shoulder, 1-DOF elbow, and 3-DOF wrist.

2. Dynamics and Payload: The arm needs to lift the UAV, which has a maximum weight of $2 \mathrm{~kg}$. The actuators must thus be able to support the static loads of the arm and the payload in all configurations with a sufficient safety margin to also allow dynamic motions.

3. Interaction and Robustness: Variable stiffness joints have been chosen to allow the arm to interact with the environment in a physically compliant, versatile, and robust way. The VSAs ensure an intrinsically safe interaction and mechanically controllable compliance, when set to appropriate stiffness values.

4. Weight and Dimensions: Because the arm and ground rover need to be transported for the search and rescue operations, the arm needs to fold away inside a small volume and should have a light weight below $15 \mathrm{~kg}$, while still achieving a reach of approximately $1 \mathrm{~m}$.

\subsection{Kinematic Analysis}

The kinematic properties of a manipulator are fundamental for the design and analysis of its performance, as they determine which motions the arm is able to execute. In this section the workspace and the achievable workspace stiffness of the manipulator are analyzed. It is verified that the arm's kinematics enable it to reach all desired positions, and investigated how the desired adjustable workspace stiffness can be achieved with 
Table 3.1: The Denavit-Hartenberg convention is a description of the kinematic relation between the links of a kinematic chain, connected by 1 DOF rotational or prismatic joints. It provides a formalism to identify joints and links, attach reference frames to the links, and to identify four parameters per joint, the link length $r_{i}$ and link twist $\alpha_{i}$, and the joint offset $d_{i}$ and joint angle $\theta_{i}$.

\begin{tabular}{|c|c|c|c|c|c|c|c|c|}
\hline Link \# & 1 & 2 & 3 & 4 & 5 & 6 & 7 & 8 \\
\hline \hline$r_{i}[\mathrm{~mm}]$ & 0 & 0 & 0 & 120 & 0 & 0 & 0 & 130 \\
$\alpha_{i}$ & $\pi / 2$ & $-\pi / 2$ & $\pi / 2$ & 0 & $\pi / 2$ & $-\pi / 2$ & $\pi / 2$ & 0 \\
$d_{i}[\mathrm{~mm}]$ & 160 & 0 & 450 & 0 & 0 & 350 & 0 & 0 \\
$\theta_{i}$ & $q(1)$ & $q(2)$ & $q(3)$ & $q(4) / 2$ & $q(4) / 2$ & $q(5)$ & $q(6)$ & $q(7)$ \\
\hline
\end{tabular}

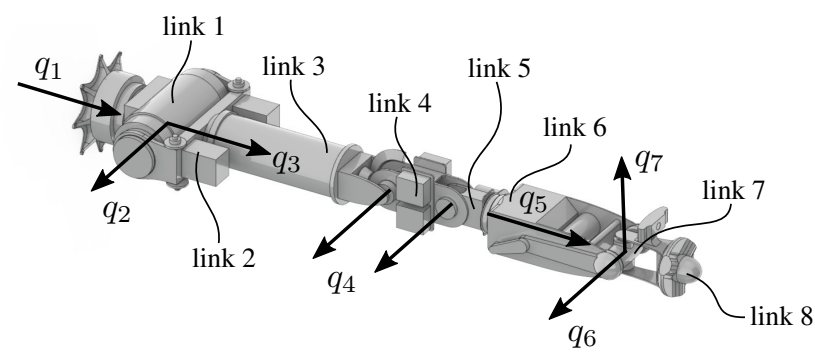

Figure 3.2: The kinematic structure of the SHERPA indicating the links and DOFs.

a minimal number of variable stiffness joints. While this section deals with the quasistatic motions and compliance of the end-effector, a static and dynamic analysis of the actuator loads is included in Section 3.4.1.

\subsubsection{Kinematic Structure}

Six DOFs are necessary for a manipulator to reach any end-effector configuration, i.e. pose and orientation, in three-dimensional space. A seventh, redundant, DOF can result in a null space, which can be used for instance to avoid obstacles or kinematic singularities, and is of special interest for Cartesian impedance control [128], if the manipulator is not in a singular configuration. Of the seven actuated DOFs of the SHERPA arm, three are located in the shoulder, one in the elbow, and three in the wrist, resulting in a roughly anthropomorphic kinematic structure, which is useful for many tasks in human-centered environments. To enable the arm to fully fold into its transport position, and to enlarge its workspace, the elbow joint consists of two axes connected by an intermediate link, driven by a single actuator. The arm's DenavitHartenberg parameters, which define the manipulator's kinematics, are given in Table 3.1, while Figure 3.2 shows an overview of the robot's kinematic structure, outlining the arm's DOFs and links. 


\subsubsection{Workspace Analysis}

The workspace of a serial-chain robotic manipulator with $n$ DOFs is defined by the set of all points that can be reached by its end-effector, and can be obtained by solving the forward kinematics problem for all permissible joint positions. This problem is to find the end-effector configuration $y \in \mathcal{Y} \subset S E(3)$ in the Cartesian workspace $\mathcal{Y}$ for a given element $q \in \mathcal{Q} \subset \mathbb{R}^{n}$ of the allowed joint positions $\mathcal{Q}$. The joint variables $q$ hereby form a set of generalized coordinates. The forward kinematic map is thus defined as $f(q): \mathcal{Q} \rightarrow \mathcal{Y}$, such that

$$
y=f(q)
$$

In practice, this means finding a transformation from the coordinate frame attached to the base of the manipulator, to one attached to the end-effector, often called the tool frame. This can for instance be achieved by concatenating homogeneous transformations between the individual links, as described in Chapter 5. The size and shape of the workspace of robotic manipulators is a decisive factor for their analysis and design, and its boundary curves or surfaces can be obtained through graphical, analytical, or numerical methods [129], and a multitude of approaches has been presented in the literature.

For determining the distance at which the manipulator can still reach a landed UAVs, the boundary curve of the workspace with downward facing end-effector (to grasp the UAV) was obtained analytically, as illustrated in Figure 3.3a.

In order to verify that the manipulator can reach the docking position, the inverse kinematics problem was solved for this position, which is to find desired joint configurations $q^{*}=f^{-1}\left(y^{*}\right)$, which result in the desired end-effector position $y^{*}$, showing that it is indeed reachable. In order to avoid taking the (pseudo-) inverse of a possibly singular function, the closed-loop Jacobian transpose scheme [130] was used here [131]. Intuitively, this scheme applies a virtual generalized force $\tau=J(q)^{T} W\left(H_{y^{*}}^{y}\right)$ to the manipulator's joints, with the geometric Jacobian $J(q)$, and the wrench $W\left(H_{y^{*}}^{y}\right)$, which depends on the homogeneous transformation $H_{y^{*}}^{y} \in S E(3)$ between the desired and the actual end-effector positions $y^{*}$ and $y$, akin to a virtual stiffness.

The link lengths and joint orientations and limits of the arm have been iteratively chosen according to this analysis, while also assuring that the arm can fold into a compact volume, as shown in Figure 3.3b.

It was also investigated if a landing platform could be placed on the rover, in order to provide the UAVs a safe and level surface to land. Such a platform could also be used as visual reference to help the UAVs localize the rover. Figure $3.3 \mathrm{~d}$ shows a possible configuration for such a platform. The idea was, however, abandoned, as its implementation would add further complexity to the system, and because the arm is already capable of retrieving landed UAVs. 


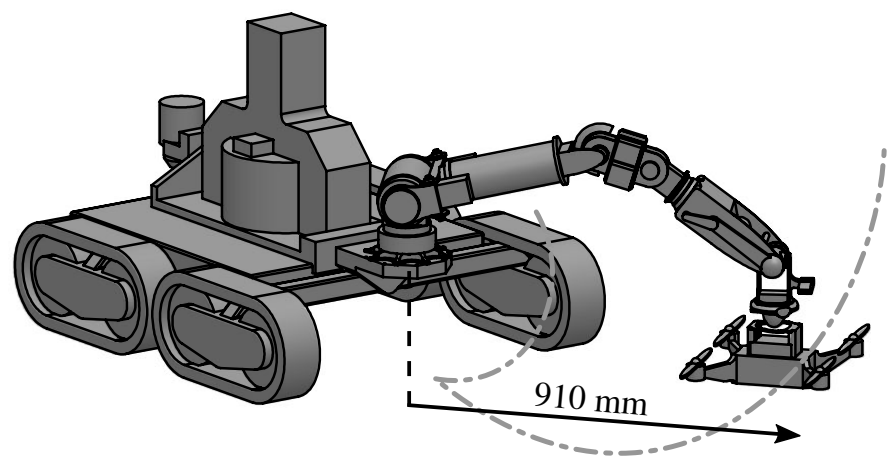

(a)

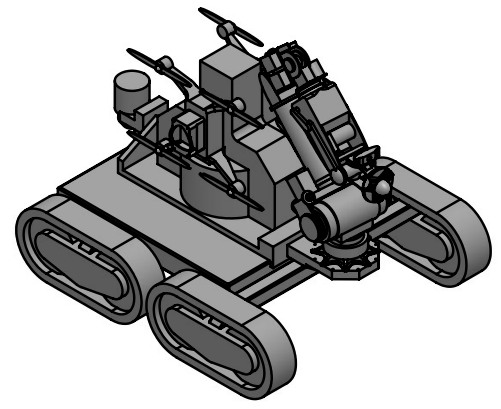

(b)

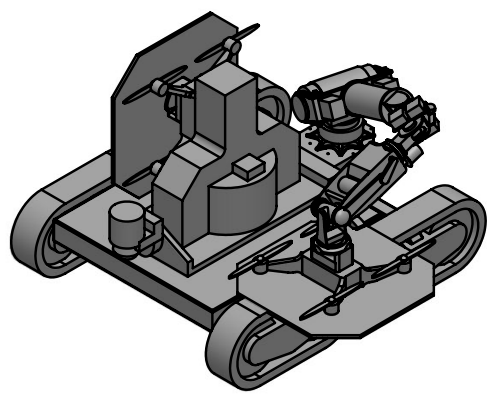

(d)

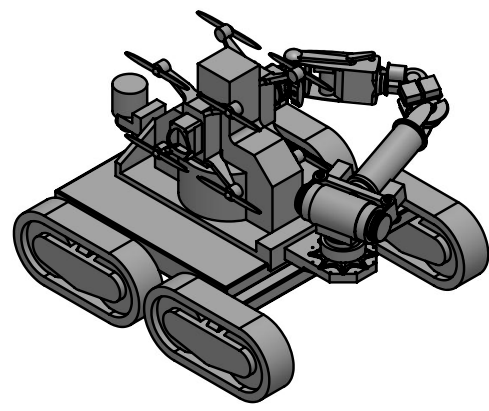

(c)

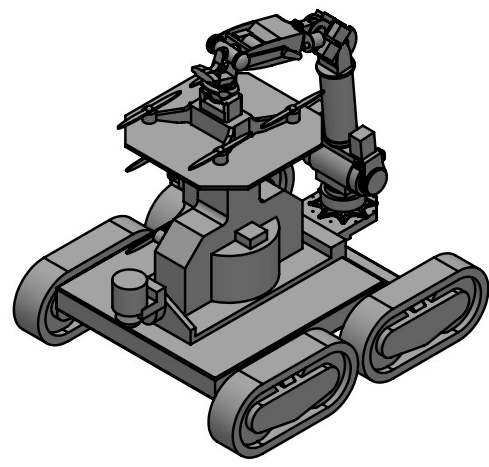

(e)

Figure 3.3: Workspace analysis for the SHERPA arm. Figure 3.3a shows the boundary curve of the workspace for picking up a landed UAV. Figure $3.3 \mathrm{~b}$ shows the arm stowed in a compact transport pose. Figure $3.3 \mathrm{c}$ shows that the arm can easily reach the docking position of the UAVs on the service station. A landing platform for the UAV can be placed on the side of the rover, as shown in Figure 3.3d, where it could fold up to protect the UAV during transport. A landing platform mounted on top of the service station, shown in Figure 3.3e, however, is not feasible, as the top of the platform approaches or exceeds the limits of the arm's workspace. 


\subsubsection{Workspace Compliance}

Robotic tasks are commonly defined in the Cartesian workspace $\mathcal{Y}$, as goal configurations can be intuitively defined through homogeneous transformations $H_{y^{*}}^{0} \in S E(3)$ between a reference frame and the desired end-effector configuration, as presented in more detail in Chapter 5. If a certain compliance is required for the execution of a task, it may be defined as the end-effector's center of compliance. To achieve different desired workspace compliances in different poses, the SHERPA robotic arm implements mechanically tunable compliance in the joint space $\mathcal{Q}$. In this subsection, the achievable workspace compliances are analyzed in relation to the different joint compliances and arm configurations.

The compliance matrix $C$ describes the linear mapping from the end-effector wrench $W:=\left(m^{T} f^{T}\right)^{T}$ to the infinitesimal end-effector twist $\delta T:=\left(\delta \theta^{T} \delta p^{T}\right)^{T}$, i.e.,

$$
\left(\begin{array}{c}
\delta \theta \\
\delta p
\end{array}\right)=\underbrace{\left(\begin{array}{cc}
C_{o} & C_{c} \\
C_{c}^{T} & C_{t}
\end{array}\right)}_{C}\left(\begin{array}{c}
m \\
f
\end{array}\right)
$$

where $C_{o}$ is the rotational compliance, $C_{t}$ the translational compliance, and $C_{c}$ the coupling compliance, coupling rotational forces and translation displacements and vice versa. In the equilibrium position, the stiffness of the manipulator, i.e. the inverse of its compliance, is described by a $(0,2)$ tensor which is the Hessian of the potential function.

The joint compliance matrix is defined in the equilibrium position as $C_{Q}=-\frac{\partial q}{\partial \tau}$, where $\tau$ are the joint torques, and $q$ the joint positions. where $q \in \mathbb{R}^{n}$ are the joints positions, and $\tau \in \mathbb{R}^{n}$ are the collocated joint torques.

Assuming infinitesimal end-effector twists around the equilibrium position $\delta T$, the relation between the joint compliance $C_{Q}$ and the Cartesian end-effector compliance $C$ is

$$
C=J(q) C_{Q} J(q)^{T}
$$

with the geometric Jacobian $J(q)$. Note that the compliance $C$ depends on both the joint compliance $C_{Q}$ and on the arm's pose $q$.

During interaction tasks the arm is required to tune the end-effector compliance normal and parallel to the plane of contact, i.e. to make the contact stiff or compliant in these directions. It was empirically analyzed which joints need to be capable of adjusting their stiffness in order to achieve the desired range of workspace compliances in three poses of special interest for the SHERPA mission: the reach (for ground grasping), catch (for aerial grasping), and dock pose (for docking). It was found that three compliant joints are sufficient to reach the desired range of the workspace compliance; the shoulder joint $q_{2}$, and the most distal wrist joints $q_{6}$ and $q_{7}$, with a coupled 2-DOF output stiffness.

A formal approach for such an analysis is presented in [132], where a methodology for modeling compliant systems with graph theory and the port-Hamiltonian 


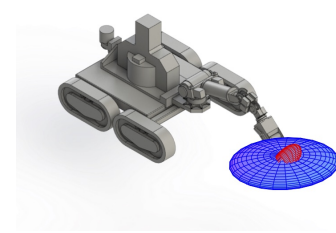

(a) reach-pose

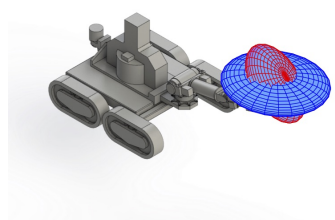

(b) catch-pose

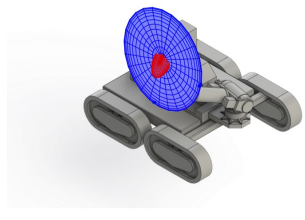

(c) dock-pose

Figure 3.4: The arm's end-effector compliance in selected poses. Two compliance ellipsoids are shown in each pose, illustrating the range of different end-effector compliances achievable through varying the compliance of the joints. In the above cases the joint compliances have been varied between $1 \cdot 10^{-3}$ and $5 \cdot 10^{-3} \mathrm{rad} / \mathrm{Nm}$ in the shoulder, and $4 \cdot 10^{-2}$ and $2.5 \cdot 10^{-1} \mathrm{rad} / \mathrm{Nm}$ in the wrist joints. This has shown to change the end-effector compliance from being low in the direction of contact and large in the orthogonal direction to the opposite case, resulting in oblate and prolate ellipsoids.

formalism are used. It is combined with an optimal control law in [133], which makes it possible to efficiently explore a large design space in terms of actuation topology and stiffness, and to optimize stiffness distributions of complex robotic systems.

Figure 3.4 shows a range of possible manipulator compliances in the selected poses for different joint stiffnesses. To better visualize the (symmetric) translational end-effector compliance, it can be plotted as an ellipsoid, obtained through its singular value decomposition $C_{t}=U \Sigma U^{T}$, where the singular values of $C_{t}$, contained in $\Sigma$, are the lengths of the semi-axes of the ellipsoid, and the columns of the matrix $U$ are the axes' orientations. The ellipsoids illustrate the capacity to achieve different end-effector compliances with the chosen actuation topology. By varying the compliance in the wrist and shoulder, it is for instance possible to control the end-effector to be compliant in one axis and rigid in the perpendicular directions and vice versa. Chapter 5 presents a more theoretical approach to determine each joint's contribution to the end effector compliance.

\subsection{Mechanical Design}

This section presents the mechanical design of the SHERPA arm, and describes the design of the shoulder, elbow, and wrist joints, as well as the gripper and the system identification, after deriving the actuator requirements.

\subsubsection{Actuator Requirements}

In order to properly dimension the actuators and gearboxes, which are the core components of the manipulator's joints, the static and dynamic loads and velocities need to be assessed. The static joint torques result from the combined gravity load of the manipulator itself and its required payload of $2 \mathrm{~kg}$, and can easily be determined. As 


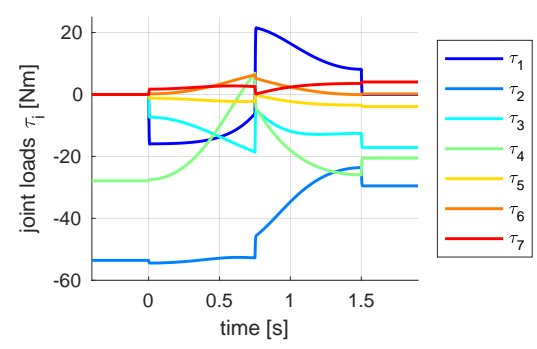

(a) Dynamic joint torques

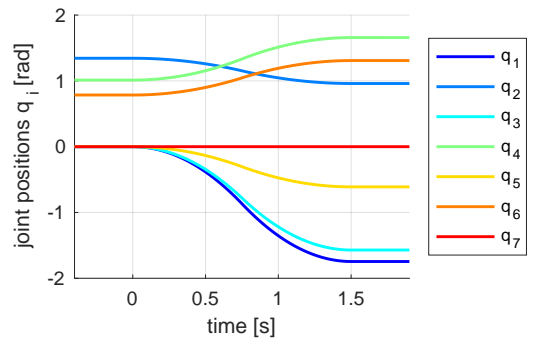

(b) Dynamic joint positions

Figure 3.5: The joint torques and positions during a simulated dynamic task. These results are used to estimate if the actuators are properly dimensioned to perform dynamic tasks. While the static loads can easily be determined, the dynamic joint torques are highly dependent on the chosen trajectory, payload, and speed of the task. For this simulation a payload of $2 \mathrm{~kg}$, the mass of the UAV, was moved by the arm from the reach position, in which it would be picked up, to the docking position on the rover in $1.5 \mathrm{~s}$.

the static loads depend on the configuration of the manipulator, the most unfavorable pose (i.e. an outstretched arm) is assumed here for each joint. With these static joint torques $\tau_{s}$, and the nominal joint torques $\tau_{n o m}$, which result from the nominal motor torques, gearbox efficiency and transmission rate, and in some cases the differential coupling of two joints, a static safety factor $S_{s}=\frac{\tau_{n o m}}{\tau_{s}}$ is defined as measure for the joints' performance. This factor must be greater than one in all cases, as otherwise it would imply that the arm cannot support the required payload in all configurations.

The analysis of the dynamic loads, however, has more variables. It depends on the chosen task, trajectory, and time in which the task is to be completed. The docking operation, which entails moving the UAV from the reach to the dock position, is selected here as a representative task for the dynamic analysis. This analysis is used to obtain an overview of how the dynamic loads compare with the nominal joint torques. The analysis is simplified by ignoring path-planning problems, and assuming a constant acceleration, resulting in linear joint velocity and parabolic joint position profiles $^{1}$. The time to execute the task is chosen as 1.5 seconds, which represents a highly dynamic motion, and enables quick docking and servicing operations, well below the time the docking station needs to exchange the UAV's battery. Figure 3.5 shows the results of the simulation of this exemplary docking task. The peak dynamic joint torques $\tau_{d}$ can now be used to define a dynamic safety factor $S_{d}=\frac{\tau_{n o m}}{\tau_{d}}$, which serves as measure of how easily the manipulator can perform this given task.

Even though these static and dynamic analyses may underestimate the required torques, for instance for aggressive control action, the dimensioning of the manipulator's actuators still remains on the safe side, as the peak or stall torques of the

\footnotetext{
${ }^{1}$ While these assumptions may not yield accurate results for all tasks, trajectories, and speeds, this simulation nonetheless shows that the arm is capable of highly dynamic operations in a representative task. The actuators furthermore remain below their nominal continuous torques, and retain their peak torque capacity for more aggressive intermittent control action.
} 
Table 3.2: Torques and Velocities of the Joints

\begin{tabular}{|l|c|c|c|c|c|c|c|}
\hline Joint & $q_{1}$ & $q_{2}$ & $q_{3}$ & $q_{4}$ & $q_{5}$ & $q_{6}$ & $q_{7}$ \\
\hline \hline Motor Power [W] & 241 & 430 & 430 & 180 & 50 & 20 & 20 \\
Torque [Nm] & 71 & $214^{4}$ & $214^{4}$ & $75^{5}$ & 10 & $8.6^{4}$ & $8.6^{4}$ \\
Static Safety $^{1}$ & 1.8 & 3.3 & 3.3 & 1.4 & 1.9 & 1.6 & 1.6 \\
Dynamic Safety $^{2}$ & 3.3 & 3.9 & 11.5 & 2.7 & 2.6 & 1.4 & 2.2 \\
Velocity $^{3}\left[{ }^{\circ} / \mathrm{s}\right]$ & 318 & 174 & 174 & $140^{5}$ & 252 & 223 & 223 \\
Limits $\left.^{\circ}{ }^{\circ}\right]$ & \pm 180 & \pm 90 & \pm 180 & \pm 180 & \pm 180 & \pm 90 & \pm 90 \\
\hline
\end{tabular}

1 The static safety $S_{s}$ refers to the nominal joint torque over the maximum static joint load.

2 The dynamic safety $S_{d}$ refers to the nominal joint torque over the maximum dynamic joint load occurring during a simulated task in which the arm moves a payload of $2 \mathrm{~kg}$ from the reach to the docking position in $1.5 \mathrm{~s}$.

3 The velocities refer to the link velocities at nominal actuator speeds.

4 The shoulder and wrist torques are the resulting torques of two differentially coupled actuators.

5 Torque and velocity at the distal elbow link.

actuators are approximately three times as large as the nominal or continuous torques for the motors in the shoulder and elbow, and even circa nine times higher for the Maxon motors in the wrist.

Table 3.2 details the specifications for the arm's joint torques and velocities, showing the nominal joint torques and velocities, along with the static and dynamic safety factors $S_{s}$ and $S_{d}$, motor power, and joint position limits.

\subsubsection{Shoulder Joint}

The arm's shoulder is a 3-DOF joint with a yaw-pitch-yaw configuration, as illustrated in Figure 3.2. The first DOF is driven by a $241 \mathrm{~W}$ Kollmorgen RBE 02210-A that is housed in the base of the arm. The second and third DOF are actuated by two differentially coupled $430 \mathrm{~W}$ ILM $85 \times 13$ RoboDrive motors [134] with CSD-32-100 Harmonic Drive transmissions [135].

The coupling of these joints is achieved through a tendon and pulley system , and results in the following transmission from actuators $a_{i}$ to joints $q_{i}$ (assuming rigid transmissions)

$$
\left(\begin{array}{l}
q_{1} \\
q_{2} \\
q_{3}
\end{array}\right)=\left(\begin{array}{ccc}
1 & 0 & 0 \\
0 & 1 / 2 & -1 / 2 \\
0 & 1 / 2 & 1 / 2
\end{array}\right)\left(\begin{array}{l}
a_{1} \\
a_{2} \\
a_{3}
\end{array}\right)
$$

The second distinguishing feature of the shoulder is the variable stiffness element placed in series with $q_{2}$. The SHERPA arm's variable stiffness joints are based on a lever arm mechanism to achieve a variable transmission between the internal springs and the output [104].

Figure 3.7 shows the shoulder's variable stiffness mechanism, in which the lever connects a polymer leaf-spring to the output, as presented in Chapter 2. By chang- 

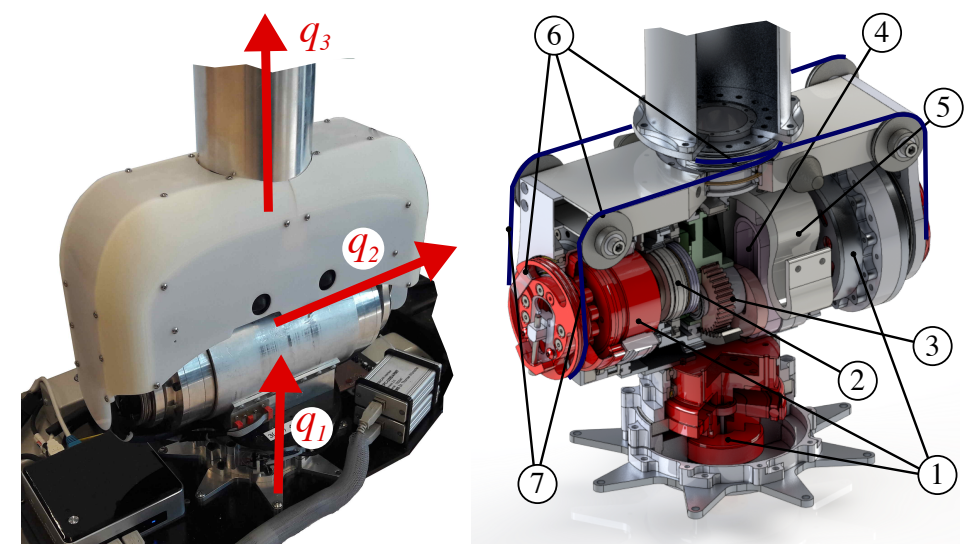

Figure 3.6: The 3-DOF shoulder joint with variable stiffness mechanism showing the DOFs and (1) joint actuators, (2) stiffness adjustment actuators, (3) stiffness adjustment mechanism, (4) lever mechanism with (5) spring, (6) pulleys, and (7) tendons.

ing the position $p$ of the pivot point, which is actuated through a hypocycloid gear train, along the lever of length $l$, the lever arm ratios of spring and output change. As further elaborated in [82], a deflection $\phi$ of the output approximately results in a deflection $s=(l / p-1) R \phi$ of the spring, and eventually in an output torque $\tau=((l / p-1) R)^{2} k_{s} \phi$ with output radius $R$, and spring stiffness $k_{s}$. Accordingly, the approximate output stiffness $K$ of the joint is $K=\partial \tau / \partial \phi=((l / p-1) R)^{2} k_{s}$.

The internal springs have been designed according to the methodology presented in Chapter 2 and [82], which optimizes the springs with respect to the energy that can be stored inside a given actuator volume. In this way, the shoulder spring was designed to enable a large deflection, even at the high loads experienced in this joint.
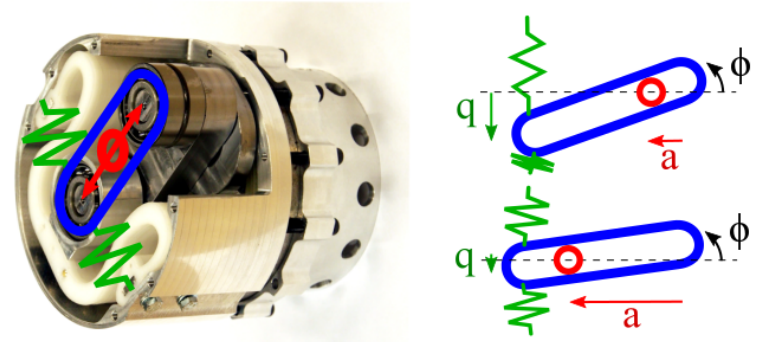

Figure 3.7: The variable stiffness module of the shoulder joint: The lever arm (blue) connects the internal polymer spring (green) to the output, through a variable transmission that is defined by the position of the movable pivot point (red) of the lever. An output deflection $\phi$ causes a larger spring deflection $s$ in a stiff configuration with a small $p$. 


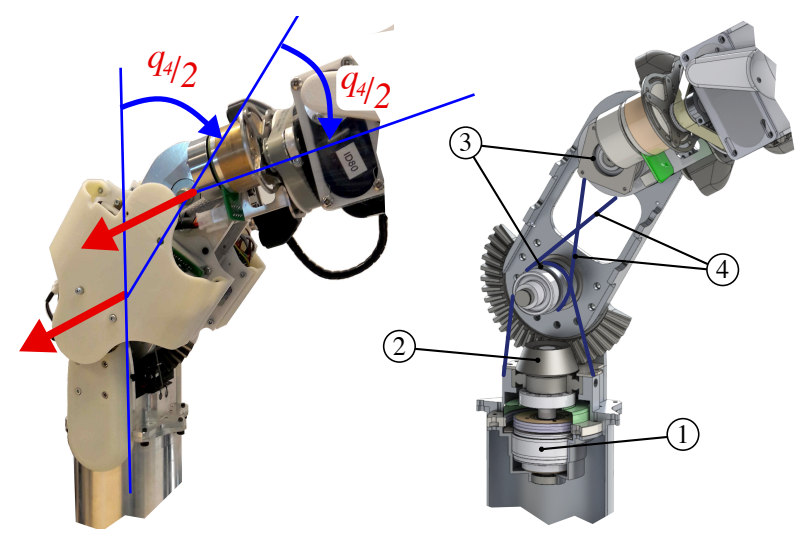

Figure 3.8: The 1-DOF elbow joint showing the coupled DOF $q_{4}$ with two rotation axes and a section view with (1) actuator, (2) bevel gear transmission, (3) pulleys, and (4) tendons. The elbow link is directly actuated through the bevel gear, while the motion of the lower arm is constrained by the tendon mechanism.

\subsubsection{Elbow Joint}

The elbow joint $q_{4}$ consists of two axes, which are connected through an intermediate link, but is driven by a single actuator. The elbow's proximal link is actuated by a 180 W ILM $50 \times 14$ RoboDrive motor [134], CSD-25-100 Harmonic Drive gearbox [135], and a 1:3 bevel gear transmission. The distal link is constrained through a tendon mechanism to follow the same angle $q_{4 b}$ (w.r.t. the distal link), as the angle $q_{4 a}$ between the distal link and the upper arm, as shown in Figure 3.8, such that $q_{4}=q_{4 a}+q_{4 b}=2 q_{4 a}$. This gives the elbow its large range of motion of $\pm 180^{\circ}$, and also allows the arm to fold into a very compact transport position.

\subsubsection{Wrist Joint}

The wrist joint also has 3-DOF, in which the last two DOF, $q_{6}$ and $q_{7}$, shown in Figure 3.9, are differentially coupled. This joint has a yaw-pitch-yaw configuration, leading to the same kinematic structure as described in Equation 3.4, but with a different orientation of the joint axes. Figure 3.10 furthermore shows the particular transmission of each of the drivetrains leading to $q_{6}$ and $q_{7}$, which represents the main novelty exploited in this joint.

The DOF $q_{5}$ is actuated with a $50 \mathrm{~W}$ Maxon EC45 flat motor and GS45-A gearbox, with an additional 18:90 pulley transmission, while two $20 \mathrm{~W}$ Maxon RE25 motors with GP-32-C gearboxes drive $q_{6}$ and $q_{7}$ through a differential coupling and a drive that includes a second variable stiffness mechanism [136].

A coupled stiffness adjustment mechanism, based on varying lever arm ratios as elaborated in Section 3.4.2, is implemented in the wrist, requiring only one actuator to adjust the output stiffness in both joints $q_{6}$ and $q_{7}$. This is made possible by fixing 
the stiffness adjustment mechanism, as well as the lever arm mechanisms including the springs, of both joints to the lower arm, and differentially coupling the respective actuators to their variable stiffness elements, and outputs leading to the joints. The principal components of the wrist mechanism are highlighted in a section view in Figure 3.9 for reference. A planetary gear system is used as 3-port differential in order to incorporate the variable stiffness mechanisms into the drive train in this way. The levers, i.e. the output of the variable stiffness modules, are connected to the ring gears and the driving actuators to the sun gear, such that the planet carrier functions as output. The two drivetrains are then in turn differentially coupled to each other through a bevel gear mechanism, to actuate the DOFs $q_{6}$ and $q_{7}$.

Due to the differential coupling of the planetary gear system, illustrated in Figure 3.10 , the angular velocity $\omega_{p}$ of the planet carrier is obtained by summing the contributions of the angular velocities $\omega_{s}$ and $\omega_{r}$ of the sun and ring gears, respectively:

$$
\omega_{p}=\frac{\left(\omega_{s} \cdot \frac{d_{s}}{2}\right) / 2}{\left(d_{s}+d_{p}\right) / 2}+\frac{\left(\omega_{r} \cdot \frac{d_{r}}{2}\right) / 2}{\left(d_{s}+d_{p}\right) / 2}=\frac{\omega_{s} d_{s}+\omega_{r} d_{r}}{2\left(d_{s}+d_{p}\right)}
$$

A first prototype of the wrist mechanism has been presented in [137], but has been extensively redesigned for the final version of the manipulator to increase robustness and strength, and to decrease the friction present in the transmission stages.

In particular the wrist's components were rearranged with respect to the prototype in such a way to reduce the number of mechanical transmission elements, and allowed proper pretensioning of the belt drives, by aligning the variable stiffness and planetary coupling modules horizontally instead of vertically (with respect to the orientation shown in Figures 3.9 and 3.10). Combined with improved bearing arrangements this lowered the friction between the wrist's output and springs by one order of magnitude, and likewise reduced the play present in the system, adjustments that are crucial when considering the goal of using the wrist as actuator with mechanically controllable impedance, as otherwise unwanted friction and backlash would dominate the characteristics of the joint. The friction and play felt at the output further depend on the transmission ratios between the modules, which were chosen in a trade-off between low friction and play, favoring a low transmission, and a large nominal output torque, requiring a high transmission ratio. The present design namely allows for an easy adjustment of the wrist's passive torque deflection characteristics, as well as output torque and speed, by adjusting the diameters of the pulleys connecting the planetary differential with the bevel gear mechanism at the end of the wrist.

Other improvements include a reduction and better distribution of the wrist's mass, increased load capacity and mechanical robustness, and greater ease of construction and maintainability. 

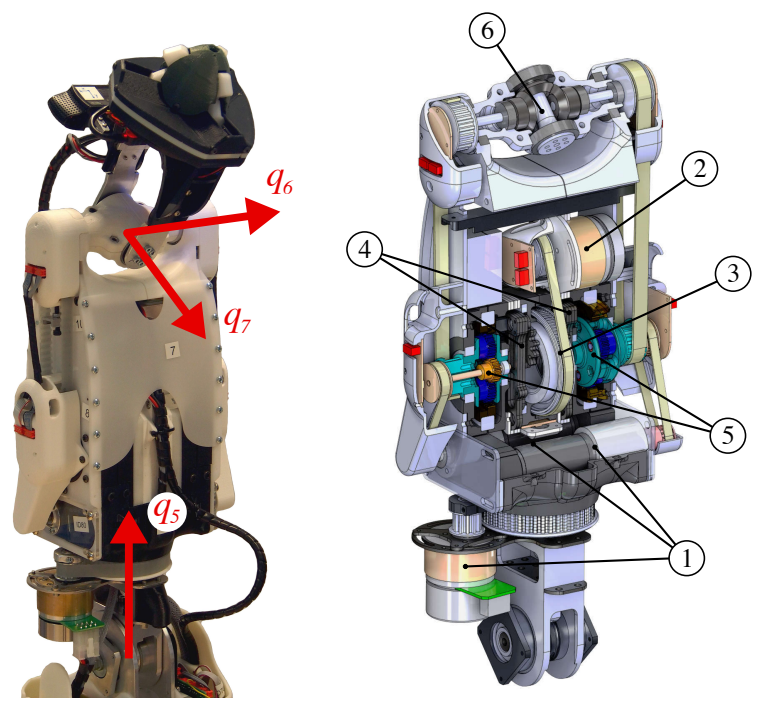

Figure 3.9: The wrist joint and end-effector showing the axes of the 3-DOFs. The section view shows the (1) joint actuators, (2) stiffness adjustment actuator, (3) stiffness adjustment mechanisms, (4) lever mechanism, (5) planetary differential, and (6) bevel differential.

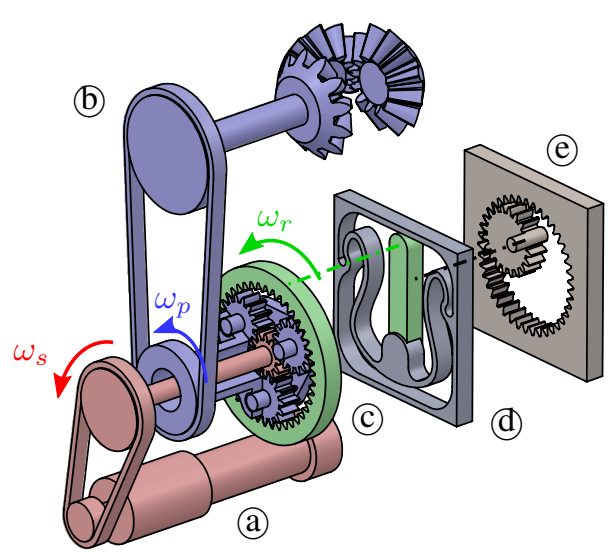

(a)

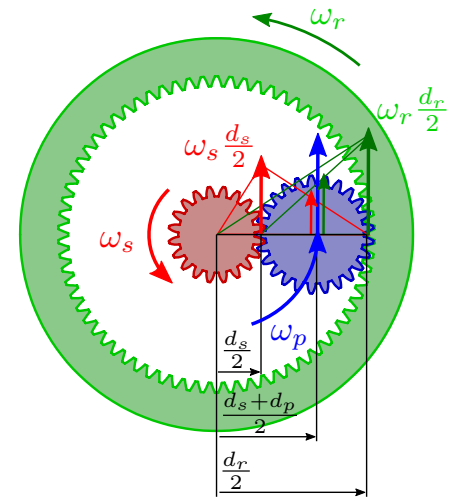

(b)

Figure 3.10: The drivetrain of one of the two most distal DOFs in the wrist joint, including the differential coupling between the actuation and variable stiffness ports. The actuators (a) and the input (b) to the differential bevel mechanism that couples the two distal wrist DOFs, are in turn coupled through a differential planetary gear set (c). The actuator is connected to the sun gear, the output to the planet carrier, and the variable stiffness stage (d) to the ring gear. The actuators, variable stiffness, and stiffness adjustment stage (e) are all fixed to the wrist, and not mounted in series, as is usually the case in comparable VSAs. Figure $3.10 \mathrm{~b}$ shows how the angular velocities $\omega_{s}$ and $\omega_{r}$ contribute to the resulting angular velocity $\omega_{p}$ of the planet carrier. $\omega_{p}$ is obtained by dividing the instantaneous linear velocity of the center of the planet gear by the radius $\left(d_{s}+d_{p}\right) / 2$. This velocity is the superposition of the contributions of the sun and ring gear, as shown in Equation 3.5 


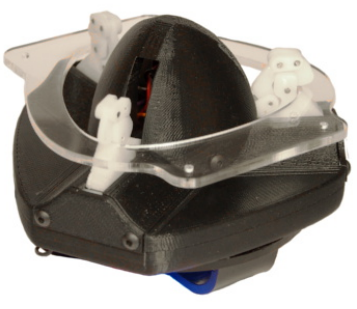

(a) Gripper

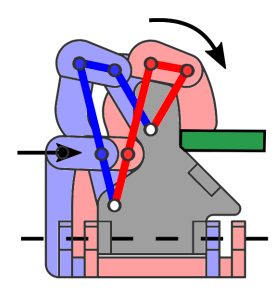

(b) Finger Mechanism

Figure 3.11: The SHERPA gripper (Figure 3.11a) and a detailed view of one of the fingers (Figure 3.11b). The fingers are built up of a linkage mechanism mounted on a moving carriage. As the carriage moves the open finger (blue) into contact with the interface (green), the linear forwards motion of the finger's extension is transformed into a rotation of the distal phalange, which wraps around the interface into the locked position (red).

\subsubsection{Gripper}

The SHERPA arm is equipped with a custom gripper with integrated actuation and electronics, which design is presented in detail in Chapter 4, and is shown in Figure 3.11. The gripper latches onto a light-weight interface attached to the UAVs, and achieves a secure form closure through a combination of a passive latching mechanism and driving dwell mechanism. The gripper engages the interface with three fingers that are extended outwards from the gripper's center through a spiral-shaped cam-mechanism and linear guides, requiring only a single actuator. Once the fingers make contact, the latching mechanism encloses the interface by means of a series of linkages.

The grasping procedure is facilitated by the gripper's shape, which guides it into the interface, and together with the arm's compliance, ensures a simple pick-up operation that is robust to small misalignments between gripper and interface. The end effector is equipped with a proximity sensor and a Logitech C920 camera for detecting the UAVs and visual servoing during grasping.

\subsubsection{System Identification}

Equation 3.3 showed that the end-effector compliance is composed of the manipulator's joint compliances. It is thus necessary to precisely identify the characteristics of the variable stiffness joints, and also to determine the elastic properties of the joints without VSAs, in order to model and control the end-effector compliance of the arm. The compliance introduced through the tendon mechanisms in the shoulder and elbow hereby are one of the main causes of additional compliance.

The joint compliances have been determined by measuring the torque-deflection characteristics of the joints with an ATI F/T Mini40 6-DOF force-torque sensor [138] and the arm's encoders [139]. Figure 3.12 shows the torque-deflection characteristics 


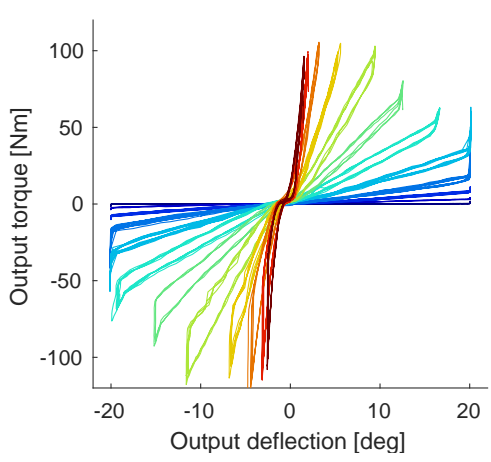

(a) Shoulder: $\tau-\phi$ plot

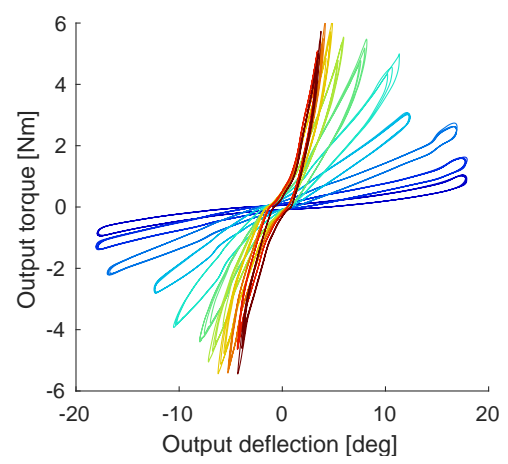

(b) Wrist: $\tau-\phi$ plot

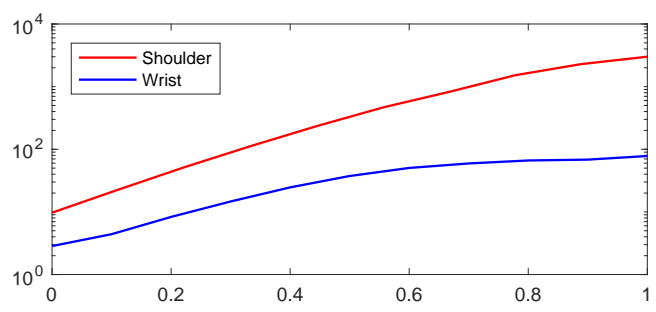

(c) Variable stiffness of shoulder and wrist

Figure 3.12: The torque-deflection characteristics of the shoulder (Fig. 3.12a) and wrist (Fig. 3.12b) joints for different stiffness settings, as well as their output stiffness (in a logarithmic scale) as a function of their relative pivot position $q / q_{\max }$ (Fig. 3.12c). Fig. 3.12b shows a one-dimensional section of the spherical 2-DOF workspace.

of the tunable compliant shoulder and wrist joints, as well as their stiffness characteristic as function of their relative stiffness setting, i.e. most compliant to most stiff pivot position.

\subsection{Electronics}

An overview of the electronic hardware for the actuation, sensing, and control of the arm is given in this section.

\section{Motor drivers}

The actuators are controlled locally in position or velocity mode, as determined by the high-level control, through Elmo Whistle miniature digital servo drives [140] that use feedback from incremental motor encoders. The actuator of the gripper is controlled 
with an Atmel ATmega328 microcontroller and Allegro A4953 motor driver, due to its low power requirements.

\section{Sensors}

In addition to incremental motor encoders on every actuator, every DOF is equipped with 14-bit absolute magnetic angular position sensors [139]. In combination with the known torque-deflection characteristics of the compliant joints, this enables a torque estimation. The arm is also equipped with mechanical limit switches, that are directly connected to the Elmo motor controllers, and act as an additional safety layer by disabling an actuator when it exceeds its allowed mechanical limit and triggers the corresponding sensor.

\section{Power Supply}

The power is supplied through a $48 \mathrm{~V}$ and $24 \mathrm{~V}$ power bus from the batteries of the SHERPA rover. Electrolytic capacitors are mounted on the interface PCBs of the motor drivers to stabilize the power lines against the counter-electromotive force of the motors.

\section{Communication}

The motor controllers are connected via the standard industrial CAN bus, a serial bus which uses four multiplexed wires and can easily be connected to the Elmo controllers, while the absolute position sensors are directly connected through a separate SPI bus, eliminating the need for additional microcontrollers on the daisy-chained encoder PCBs.

\section{High-level Control}

An Intel NUC running Ubuntu 14.04. and ROS [141] Jade is used as platform for running the high-level planning and control, which allows to use many existing packages and libraries.

\subsection{Software Architecture}

An overview of the software controlling the SHERPA arm is shown in Figure 3.13, which can be grouped into several main modules.

The high-level planner coordinates the execution of the arm's tasks with the rest of the SHERPA team through the delegation module, and divides more complex compound actions, into a sequence of elementary actions, which are executed through a FSM (Finite State Machine). It uses the ROS-based planning framework MoveIt! [142] to generate collision free joint space trajectories for desired end-effector positions. 


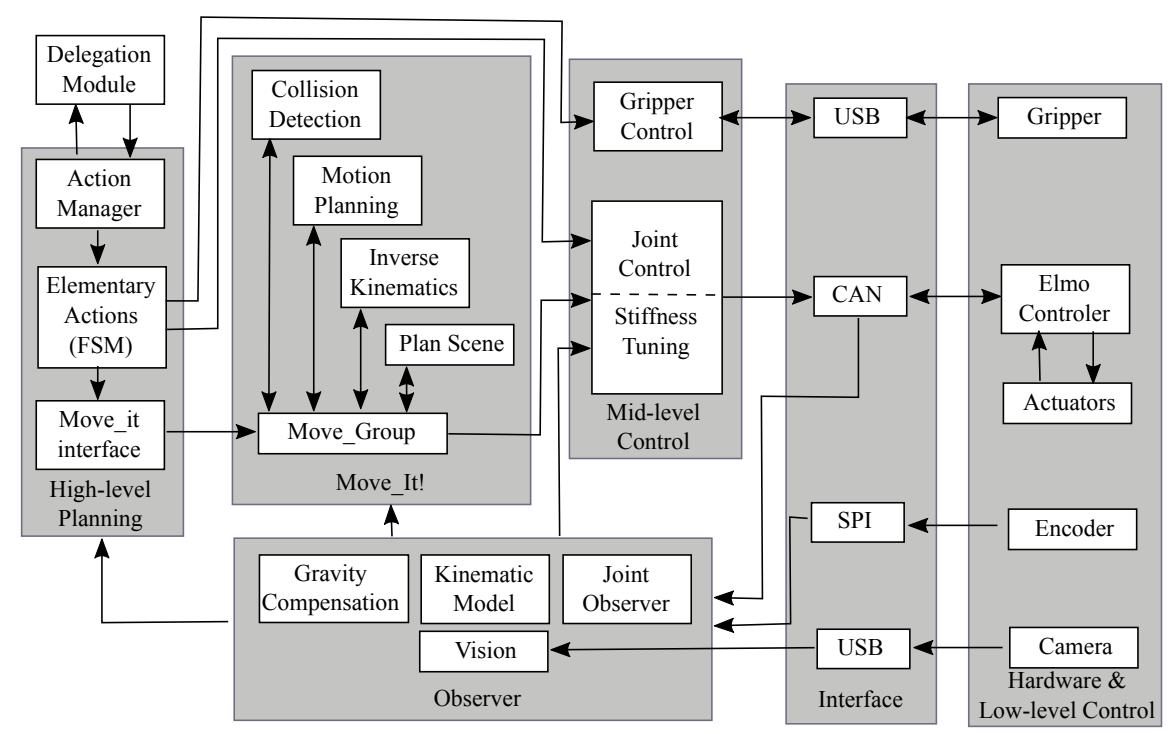

Figure 3.13: The software structure of the SHERPA arm can be divided into several main modules: a high-level planner, coordinating the arm's actions with the SHERPA team through the delegation module, a motion planner implemented in Move_It!, a mid-level control and an interface layer, the hardware and low-level control layer, and an observer.

Fast and efficient sampling based motion planning algorithms, such as RRT (Rapidlyexploring Random Trees) are utilized, and already integrated in MoveIt! as part of the OMPL [143]. The planner guarantees the transition between parts of a task-sequence and selects whether the action is carried out in position, velocity, or impedance control mode.

The mid-level controller translates the joint set-points into corresponding commands sent to the hardware interface layer, taking into account the arm's kinematics, in particular the differentially coupled joints. It is composed of controllers for the individual, multi-DOF joints, and accepts position, velocity, or torque set-points, while the low-level loop-control is provided by the ELMO motor drivers.

The observer uses feedback from all sensors, and combines it with an internal model of the arm's kinematics and joint-stiffness characteristics, to provide a feedback on the arm's state, i.e., the joint positions, velocities, and torques.

The interface layer connects the controller and observer, which communicate via ROS-topics, to the physical layer of the robot, through the CAN and SPI buses. 


\subsection{Experiments}

This section presents experiments affirming the robotic arm's suitability to complete the SHERPA mission. A first set of experiments shows the arm's capacity to modify its Cartesian workspace compliance, as required for interaction tasks. A second experiment presents the arm carrying out a battery change procedure by grasping, docking, and deploying a landed UAV.

\subsubsection{Workspace Compliance}

The arm's ability to achieve a variable Cartesian end-effector compliance, by tuning the joint stiffnesses is demonstrated by the following set of experiments. In these, the workspace compliance was measured for each of the selected robot poses illustrated in Figure 3.4, reach, catch, and dock, with two sets of joint stiffnesses in the shoulder and wrist variable stiffness modules ${ }^{2}$.

Equation 3.2 shows that when only forces $f$, but no torques $m$, are applied to the end-effector its translational displacement is $\delta p=C_{t} f$. Considering a single component of $\delta p$, e.g. the displacement $\delta x$ in $x$-direction, gives

$$
\delta x=C_{t 11} f_{x}+C_{t 12} f_{y}+C_{t 13} f_{z}
$$

Where $C_{t i j}$ are the components of the translational compliance matrix $C_{t}$. If a force $F$ applied to the end-effector only acts in one direction, e.g., the $x$-direction, such that $F=\left(\begin{array}{lll}f_{x} & 0 & 0\end{array}\right)^{T}\left(f_{y}<<f_{x}\right.$ and $\left.f_{z}<<f_{x}\right)$, then Equation 3.6 further simplifies to $\delta x=C_{t 11} f_{x}$ and we have approximated the element $C_{11}$ of the compliance matrix as $C_{11}=\delta x / f_{x}$.

Using this approach we can intuitively characterize the translational compliance $C_{t}$ by applying the set of forces

$$
\mathcal{F}=\left\{F_{x}=\left(\begin{array}{c}
f_{x} \\
0 \\
0
\end{array}\right), F_{y}=\left(\begin{array}{c}
0 \\
f_{y} \\
0
\end{array}\right), F_{z}=\left(\begin{array}{c}
0 \\
0 \\
f_{z}
\end{array}\right)\right\}
$$

and measuring the resulting small end-effector displacement. As the elements in $\mathcal{F}$ are aligned with the axes of the Cartesian reference frame, they allow to select the columns of $C_{t}$ in three measurements. We obtain $C_{t}$ as

$$
C_{t}=\left(\begin{array}{ccc}
\delta x_{x} / f_{x} & \delta x_{y} / f_{y} & \delta x_{z} / f_{z} \\
\delta y_{x} / f_{x} & \delta y_{y} / f_{y} & \delta y_{z} / f_{z} \\
\delta z_{x} / f_{x} & \delta z_{y} / f_{y} & \delta z_{z} / f_{z}
\end{array}\right)
$$

where $\delta x_{i} / f_{i}, \delta y_{i} / f_{i}$, and $\delta z_{i} / f_{i}$ are the compliances in $x, y$, and $z$ direction corresponding to force $F_{i}$.

\footnotetext{
${ }^{2}$ The Hessian of the potential function, which is the tensor describing the stiffness of the manipulator, is only defined as such in the equilibrium position. The following experiments ignore the preload of the arm by gravity, as the resulting deflections are small, and do not qualitatively influence the results.
} 


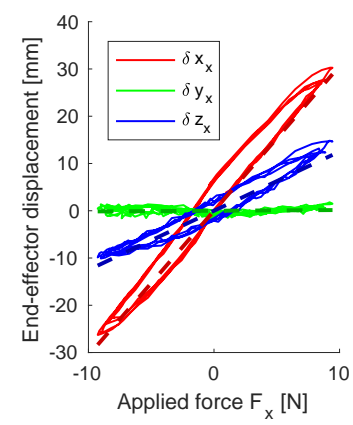

(a) $F=\left(\begin{array}{lll}f_{x} & 0 & 0\end{array}\right)^{T}$

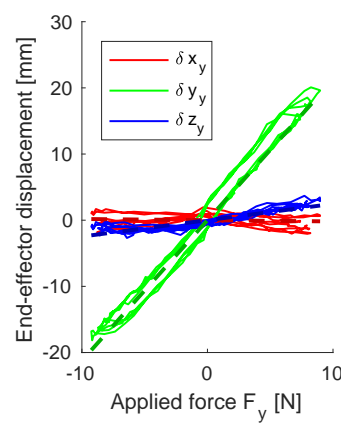

(b) $F=\left(0 f_{y} 0\right)^{T}$

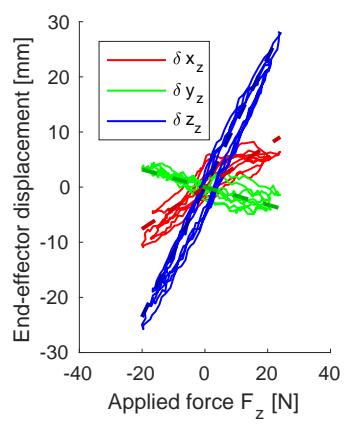

(c) $F=\left(\begin{array}{lll}0 & 0 & f_{z}\end{array}\right)^{T}$

Figure 3.14: The end-effector deflection in $x$-, $y$-, and $z$-direction in the reach-pose as function of the applied forces $\mathcal{F}:\left\{F_{x}=\left(\begin{array}{lll}f_{x} & 0 & 0\end{array}\right)^{T}, F_{y}=\left(\begin{array}{lll}0 & f_{y} & 0\end{array}\right)^{T}, F_{z}=\left(\begin{array}{lll}0 & 0 & f_{z}\end{array}\right)^{T}\right\}$ and the fitted compliances $\delta i_{i} / f_{i}$ (dashed).

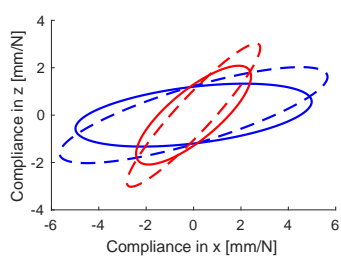

(a) Reach-pose compliance in $\mathrm{x}-\mathrm{z}$

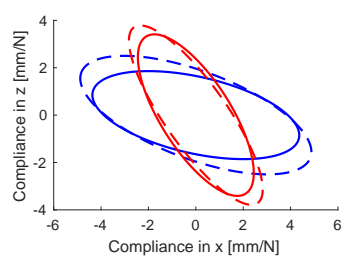

(b) Catch-pose compliance in $\mathrm{x}-\mathrm{z}$

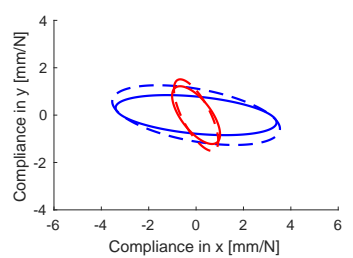

(c) Dock-pose compliance in $\mathrm{x}-\mathrm{y}$

Figure 3.15: The translational end-effector compliances in the reach, catch, and dock-poses for two different joint stiffness settings (blue and red). The figure shows compliance ellipses, which are the projection of the calculated (dashed line) and measured (solid line) compliance ellipsoids. 
These compliances have been determined by measuring the end-effector wrench with an ATI F/T Mini40 6-DOF force-torque sensor [138], that was mounted in place of the gripper. The end-effector position was determined through the arm's observer, which combines encoder measurements with an internal model of the arm, as illustrated in Figure 3.13. The compliance in a certain direction was then estimated by the slope of the end-effector displacement in the corresponding direction as a function of the applied force, by fitting a first order polynomial to the measurement data. Figure 3.14 shows an example of these measurements.

Figure 3.15 shows compliance ellipses, which are the projection of the compliance ellipsoids obtained from Equation 3.2 for the calculated, and Equation 3.7 for the measured end-effector compliance, onto the corresponding plane in which the task takes place. The measured compliances closely match the calculated values, and demonstrate that the arm is able to achieve the tunable workspace compliance described in Section 3.3.3.

\subsubsection{Battery Replacement}

The docking operation is the most important task of the manipulator during the SHERPA mission, and is therefore used here to demonstrate the arm's ability to perform the required manipulation tasks. It is part of the more complex battery change operation, which requires collaboration between the SHERPA agents, and is presented in detail in Chapter 6. Figure 3.16 shows the docking operation, in which the arm moves from the transport to the scan-pose to detect the landed UAV, before it grasps it in the reach-pose. It then moves it into the docking-pose where the battery can be exchanged, before placing it back on the ground and returning to the transport-pose. The arm's tunable compliance facilitates the successful execution of this task, as it requires dexterous interaction with the UAV and the docking station. In this experiment the mechanical end-effector stiffness was tuned for different stages of the task requiring interaction with the environment, e.g. during grasping and docking.

\subsection{Conclusions}

After analyzing the demands of the SHERPA mission, an arm design has been chosen, that combines robustness and adaptability. Experiments show that the arm is capable of accurate position and velocity control in its required workspace, as demonstrated in Section 3.7.2, as well as incorporating tunable, passive compliant joints, allowing it to tune its workspace stiffness, as demonstrated in Section 3.7.1, and to interact safely with its environment. This enables the arm to successfully execute its task during the SHERPA mission, for instance exchanging the UAV's batteries.

The dissertation's research objective RO3 has been achieved by identifying and implementing a hybrid rigid-compliant actuation topology that provides the required variable range of workspace compliances with only three joints with variable mechani- 


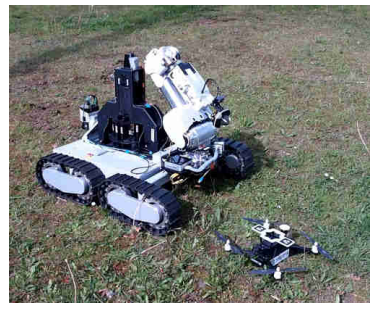

(a) Transport pose

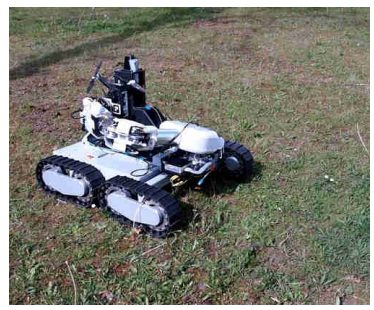

(d) Dock pose

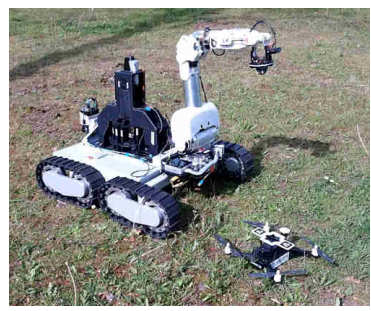

(b) Scan pose

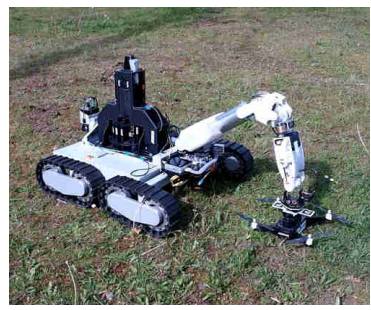

(e) Place pose

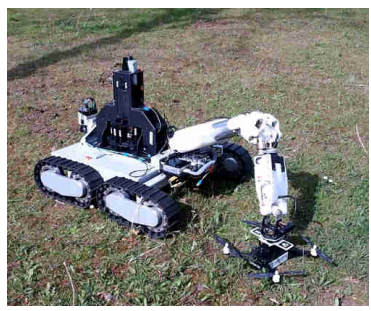

(c) Reach pose

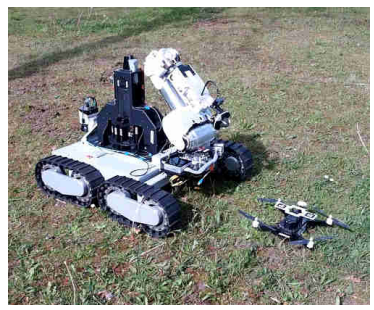

(f) Transport pose

Figure 3.16: A sequence of images showing the arm in different poses during the UAV battery exchange, specifically (a) transport, (b) scan, (c) reach, (d) dock, (e) place, and (f) transport.

cal stiffness, one VSA in the shoulder, and two joints with a coupled variable stiffness in the wrist. This solution allows to limit the overall system complexity, while still retaining the advantages of compliant manipulation.

A number of engineering objectives have also been addressed in this chapter. The chosen kinematics of the roughly anthropomorphic 7-DoF manipulator assure that all relevant poses needed to deploy, dock, and recover the UAVs can be reached, thereby accomplishing EO1.

The results presented in this chapter represent a considerable amount of design, testing and integration work carried out on both the component and system level, as required by EO2, in order to obtain a fully functional manipulator suited for the use in the SHERPA project, and as research platform, as demonstrated here by preliminary experiments.

The software and control architecture of the manipulator presented in this chapter furthermore lays the basis for addressing RO5. The manipulator's tasks are described and implemented through elementary and compound actions, that are interfaced with the overall delegation and control framework used within the SHERPA project to integrate the various agents and perform collaborative tasks, as further described in chapter 6 . 



\section{CHAPTER 4}

\section{THE SHERPA GRIPPER: GRASPING OF SMALL-SCALE UAVS}

This chapter presents the mechatronic design of a compact gripper, intended to manipulate UAVs (Unmanned Aerial Vehicles) in search and rescue missions. The gripper latches onto a custom interface, that is mounted on the aerial vehicle, and achieves a secure form closure through a combination of a passive latching mechanism and a driving dwell mechanism. The grasping procedure is facilitated by the gripper's shape, which guides it into the interface, making for a simple pick-up operation that is robust to small misalignments between gripper and interface, satisfying the requirement to reliably grasp the UAVs as defined in the engineering objective EO3. The design of the gripper is presented, and the features of its components analyzed. Successful experiments validate that the gripper meets its requirements, and presents an effective solution for grasping objects like UAVs.

This chapter has been previously published as:

E. Barrett, M. Reiling, M. Fumagalli, and R. Carloni, "The SHERPA Gripper: Grasping of Small-Scale UAVs", in Proceedings of the IEEE International Symposium on Safety, Security, and Rescue Robotics, pp. 384-389, 2016. 


\subsection{Introduction}

Emerging robotic technologies promise to revolutionize the way search and rescue activities are carried out in the future, especially in hazardous or hostile environments. In the scope of the SHERPA project [12] small scale UAVs need to be deployed, recovered, and serviced by a mobile ground rover equipped with a robotic arm, as laid out in Chapter 1. The arm must be able to reliably grasp and dock the UAVs in order to exchange their batteries and increase their operational time and autonomy.

This chapter focuses on the mechatronic design and experimental validation of the SHERPA gripper that allows the robotic arm to pick the UAVs up from where they landed and place them on the ground rover.

A multitude of different gripper designs have been developed to address the need of grasping and interacting with objects. The most advanced of these emulate the human hand and are reaching high degrees of versatility and dexterity [144, 145, 146, $147,148]$. On the other side, most industrial grippers are based on much more simple mechanisms that are suited for simple limited tasks, or use compliant underactuation to increase their range of application, while keeping their overall complexity comparatively low [149, 150].

Following this approach, a gripper is presented, that reduces the mechanical and control complexity, by engaging a custom interface mounted on the UAV, where the gripper's structure helps to guide it into a securely locked position. Such a solution, tailored to the needs of the search and rescue operation, allows the gripper to engage the UAVs with a simple, compact and robust design and grasping procedure. Even though the proposed gripper is developed for a specific UAV interface and a specific task within the SHERPA project, its operating principle can be applied to a variety of different applications. The final design of the SHERPA gripper is shown in Figure 4.1.

The remainder of this chapter is organized as follows. Section 4.2 presents the requirements of the SHERPA gripper with reference to the UAV interface. The design of the gripper starts in Section 4.3, where the general shape is described. The design of the fingers is shown in 4.4, while the actuation mechanism is presented in Section 4.5. The mechatronic implementation is described in Section 4.6 and validated in experiments as presented in Section 4.7. Finally, concluding remarks are drawn in Section 4.8 .

\subsection{Requirements}

The SHERPA gripper design should realize a self-locking mechanism on a custom lightweight ring-shaped interface, placed on the UAV. This guarantees that the gripper's actuator only consumes power while grasping or releasing the interface, and the mechanism is mechanically locked when the gripper is engaged.

The working principle should be insensitive to small misalignments between itself and the interface, and mechanically guide itself into the correct position, relaxing the 


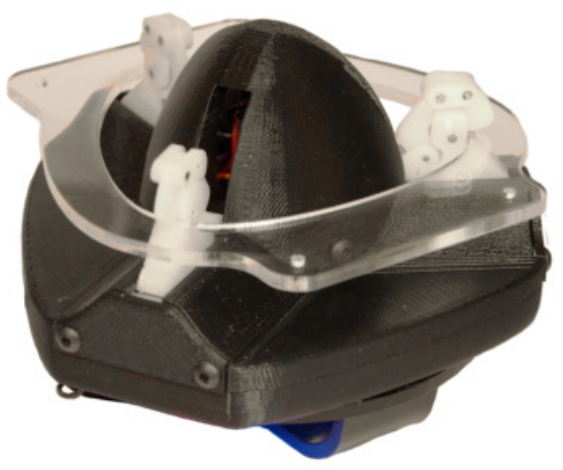

Figure 4.1: The SHERPA gripper latching onto a transparent interface.

demands on sensing and control during the pick-up of the UAV.

Moreover, the SHERPA gripper should also fulfill the following requirements:

- The gripper's payload is $2 \mathrm{~kg}$, which corresponds to the maximum weight of the UAV.

- The grasping time is below $0.5 \mathrm{~s}$, to enable the gripper to be used in dynamic scenarios.

- The gripper is lightweight, weighing about $200 \mathrm{~g}$, to reduce the load on the arm, and compact, with an overall diameter of about $110 \mathrm{~mm}$, matching the size of the interface that can be placed on the UAV.

\subsection{Engagement Mechanism}

An easy and robust engagement of the interface by the gripper is needed for a simple and reliable grasping operation. The design of the gripper needs to passively compensate misalignments between the robotic arm and the UAV, by mechanically guiding the gripper into the interface.

A cone-shaped gripper has been chosen, that engages a ring shaped interface from the inside. This makes the operation of grasping the UAVs similar to ring jousting, where a lance is passed through a ring, rather than resembling catching an object in the palm of one's hand.

The ring shaped interface is easy to mount on the UAVs and even a large ring can have a lightweight construction. Its low mass of $16 \mathrm{~g}$ is important, as a heavy interface would negatively impact the UAV's payload and battery life.

Figure 4.2 shows how the gripper engages the interface. As can be seen, the interface is not circular, but shaped slightly triangular. This provides rotational alignment and locking. 


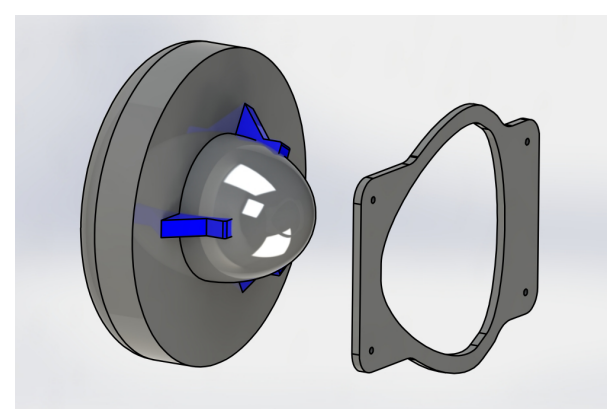

Figure 4.2: The gripper engages the interface by entering the cone-shaped tip into the ring-shaped interface. This ensures that the grasping procedure is robust against misalignments between the gripper and interface.

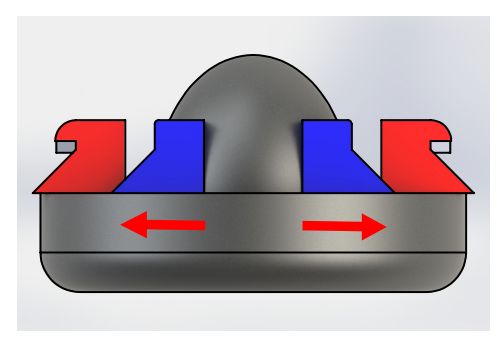

Figure 4.3: The gripper latches onto the interface by extending three fingers outwards from the center of the gripper. The fingers are shown in the open position (blue) and in the extended position (red) where they lock around the interface.

\subsection{Latching Mechanism}

The gripper has three fingers, that are extended outwards from its center to engage and lock onto the interface. A rotational locking is ensured through the slightly triangular shape of the interface ring and the three fingers, resulting in a form fit. The radial and axial locking of the connection between gripper and interface is achieved by the fingers enclosing the interface, as can be seen in Figure 4.3.

This section describes the mechanism by which the fingers passively envelop the interface as they are moved outward and into contact with it, while they are compactly contracted when the gripper enters and aligns the interface.

\subsubsection{Working Principle}

The finger mechanism is shown in Figure 4.4 and consists of a carriage supporting the phalanges, that is actively moved outwards of the center of the gripper to engage the interface. The proximal phalange can move back and forth in the carriage, but is preloaded with a spring, that keeps the distal phalange retracted, if the finger does 

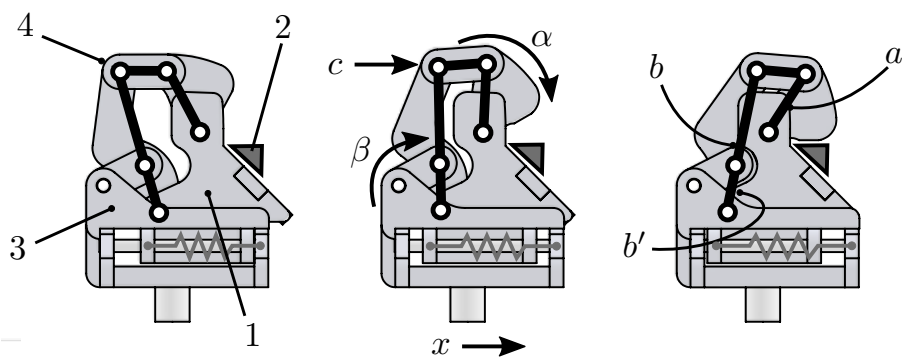

Figure 4.4: A sequence of detailed views showing a finger latching onto the interface: the proximal phalange (1) makes contact with the interface (2) as it is being moved outwards, the carriage (3) is being moved further to the right, thus engaging the linkages (4), and moving them from the retracted (left) into the latched (right) position.

not make contact with the interface. During contact, the distal phalange is forced to pivot forward and around the interface through a connection between the carriage and link $b$, at the height $b^{\prime}$. It finally encompasses the interface, so that the three fingers together securely lock the interface in all degrees of freedom.

\subsubsection{Kinematic Analysis}

An approximate kinematic analysis is presented to provide a better insight into how the mechanism's design parameters, especially the relative dimensions of the linkage mechanism, determine its performance. Translating the linear displacement $x$ of the proximal phalange with respect to the carriage into the enclosure angle $\alpha$ of the distal phalange, and the involved forces are of particular interest.

The analysis presented here uses small angle approximations for deriving the mechanism's transmission ratios, but still describes its working principle well and aids in its understanding.

The approximation $x=b^{\prime} \cdot \beta$ gives the angle by which the inner link is displaced as $\beta=x / b^{\prime}$. Together with $c=b \cdot \beta$, and the fact that the linkage mechanism imposes the same displacement $c=b \cdot \beta=a \cdot \alpha$ also on the second triangle, the transmission from the linear displacement $x$ to the angle $\alpha$, is thus

$$
\frac{\alpha}{x}=\frac{b}{a} \cdot \frac{1}{b^{\prime}}
$$

From the power continuity $\tau_{\alpha} \cdot \dot{\alpha}=F_{r} \cdot \dot{x}$ between the linear input and rotational output of the mechanism, and the resulting grasping force $F_{g}=\tau_{\alpha} / a$ at the end of the enclosing link, the transmission between the force $F_{r}$ acting radially outward on the interface, and the force $F_{g}$ with which the interface is grasped, when neglecting friction, is found to be

$$
\frac{F_{g}}{F_{r}}=\frac{b^{\prime}}{b}
$$




\subsubsection{Discussion}

Equation 4.4 makes it clear that a dwell mechanism is desirable to enable the gripper to lock into the latched position, without requiring the actuator to apply the radial force $F_{r}$ needed to maintain the grasping force $F_{g}$.

But even if this is the case, the ratio of the link lengths $b^{\prime}$ and $b$ also determines the internal loads needed to support the external loads. For a secure and rigid grasp, the linkage mechanism must be stiff and strong enough to withstand these loads. This needs to be considered when designing the mechanics and choosing the transmissions.

On the other hand, a large transmission $\alpha / x$, as given in Equation 4.1, causes the distal phalange to enclose the interface already at smaller linear displacements, such that a good trade-off should be made.

\subsection{Actuation Mechanism}

This section presents and analyses actuation mechanisms to drive the linear outwards motion of the fingers. We consider straight line mechanisms that operate with the help of a linear guide for the gripper's fingers. To keep the complexity of the gripper low, the mechanisms need to drive all three fingers with a single actuator.

The actuation of the gripper should be implemented in such a way, that it requires no actuator power to hold the interface in the latched position. It was determined in the previous section, that this requires the actuation mechanism to have a dwell point at the extended position of the fingers, i.e. $\left.\dot{r}(\dot{\theta})\right|_{r_{\max }}=0$. This means that the fingers reach a singularity in this position, and that the load on the actuator becomes zero.

There are many straight line dwell mechanisms, but most are not easily applicable for use in a compact gripper. Below we consider only two well-suited types of dwell mechanism, and analyze their stroke and the torque required to drive a payload of $20 \mathrm{~N}$ with a single finger from a distance $r_{1}=20 \mathrm{~mm}$ to $r_{2}=40 \mathrm{~mm}$ from the center of the gripper.

\subsubsection{Linkage Mechanism}

The first mechanism is a simple two-bar linkage, as shown in Figure 4.5, where the crank $a$ drives the link $b$, which end is connected to the finger, and where the load $F_{r}$ and the tangential reaction force $F_{t}$ of the linear guide are acting. The links driving the other fingers are connected to the same input as crank $a$.

The radial position of the finger is given through the function $r=a \cdot \cos \theta_{1}+b$. $\cos \theta_{2}$. From the law of sines we obtain $\theta_{2}=\arcsin \left(a / b \sin \theta_{1}\right)$ such that the radius $r\left(\theta_{1}\right)$ is a function of $\theta_{1}$ :

$$
r\left(\theta_{1}\right)=a \cos \theta_{1}+b \sqrt{1-\left(a / b \sin \theta_{1}\right)^{2}}
$$




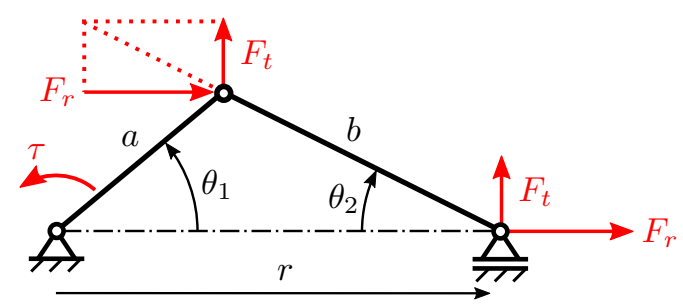

Figure 4.5: A two bar linkage mechanism. Link $a$ is actuated such that the distal end of link $b$ is at the distance $r$ from the center, while holding the radial load $F_{r}$.

The partial derivative of this kinematic function with respect to $\theta_{1}$ is the Jacobian $J\left(\theta_{1}\right)=\frac{\partial r\left(\theta_{1}\right)}{\partial \theta_{1}}$, which relates the angular velocity $\dot{\theta}_{1}$ of the actuated link to the linear velocity $\dot{r}$ of the finger, such that $\dot{r}=J \dot{\theta}_{1}$. As the transpose of the Jacobian also relates the driving torque the radial load $F_{r}$ to the driving torque $\tau$, we obtain:

$$
\tau=J^{T} F_{r}=-\left(a \sin \theta_{1}+\frac{a^{2}}{b} \frac{\sin \theta_{1} \cos \theta_{1}}{\sqrt{1-\left(a / b \sin \theta_{1}\right)^{2}}}\right) F_{r}
$$

The finger position and actuation torque are shown in Figures 4.6 and 4.6 respectively, and for different ratios of the links $a$ and $b$. Most importantly, it can be seen that the actuator torque is indeed zero at the fully extended position $r=40 \mathrm{~mm}$ of the fingers. Secondly, the effect of the ratio $a / b$ on the transmission is visible: A long link $a$ requires a higher torque but smaller angle $\theta_{1}$ to complete the required stroke of $20 \mathrm{~mm}$.

Because the same crank shaft needs to drive three fingers in the same plane, the actuation angle $\theta_{1}$ cannot exceed $120^{\circ}$, and should even be limited to approximately $90^{\circ}$ to avoid collisions with the other fingers.
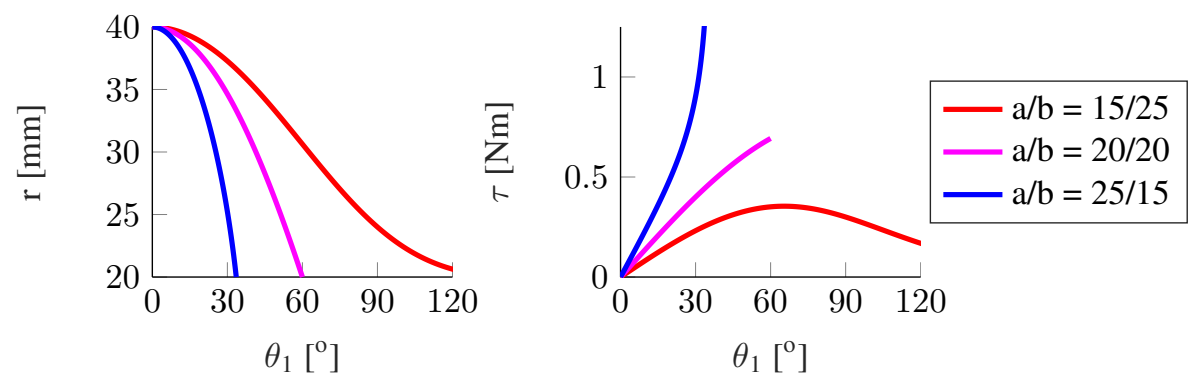

Figure 4.6: Linkage mechanism: The radial position $r$ of the fingers and the torque $\tau$ needed to move the fingers with a radial load of $F_{r}=20 N$ as function of the driving angle $\theta_{1}$ for different link lengths, ranging from $20 \mathrm{~mm}$ to $40 \mathrm{~mm}$. 


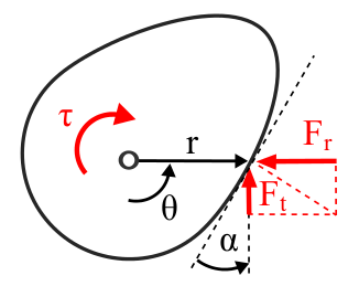

Figure 4.7: The radial load acts on the profile of the cam mechanism under the contact angle $\alpha$ and the distance $r$ from the center.

\subsubsection{Cam Mechanism}

The second actuation mechanism considered is a cam mechanism, shown in Figure 4.7, where the distance from the center is given by the profile $f$ of a cam disc:

$$
r(\theta)=f(\theta)
$$

As it is defined by the Jacobian, the actuation torque depends on the slope $\alpha$ of the profile, but can also be expressed through the tangential force $F_{t}$ occurring in the contact point:

$$
\tau=r F_{t}=r \tan \alpha F_{r}
$$

The transmission between the input rotation and the linear output motion can be determined by selecting a desired cam profile $f(\theta)$. One requirement for this curve, is that it needs to have a dwell point at the extended position of the fingers.

A linear, or Archimedean, spiral with constant pitch as cam profile leads to a constant transmission and thus to an input torque that does not depend on the position $r$ of the finger. This is advantageous, as torque peaks are avoided, and the dwell property can still be achieved by adding a small section of constant radius at the end of the profile.

The finger position for a linear spiral is:

$$
r=r_{1}+b \cdot \theta
$$

the derivative of which is simply $J(\theta)=\frac{\partial r(\theta)}{\partial \theta}=b$, and the tangens of the pitch of the linear spiral $\tan \alpha=b / r$, the torque becomes

$$
\tau=b F_{r}
$$

This constant transmission allows to use the slope, or pitch, of the spiral as parameter to better match a given actuator's characteristics, such as its torque or velocity, to the requirements of the mechanism. 

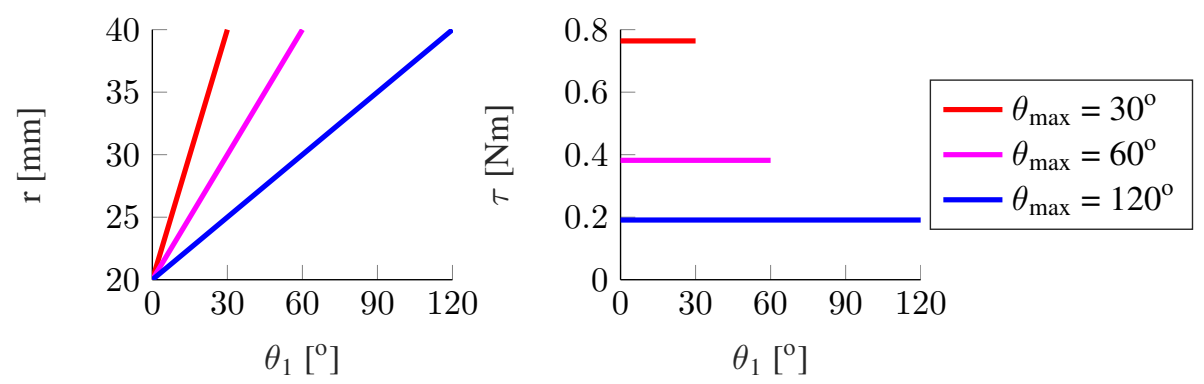

Figure 4.8: Cam mechanism: The radial position $r$ of the fingers and the torque $\tau$ needed to move the fingers with a radial load of $F_{r}=20 \mathrm{~N}$ as function of the driving angle $\theta$ for different pitch angles and corresponding $\theta_{\max }$, ranging from $20 \mathrm{~mm}$ to $40 \mathrm{~mm}$.

\subsubsection{Discussion}

Both mechanisms are suitable for actuating the gripper's fingers. However, the cam profile offers greater freedom in choosing the transmission between the actuator and fingers.

The shape of the cam profile can be precisely adapted to the needs of the mechanism, also in such a way that the profiles of the different fingers do not interfere with each other. The driving angle $\theta$ can then even be larger than the $120^{\circ}$ separating the fingers.

When choosing such a high transmission between input $\theta$ and output $r$, the torque requirements of the actuator can be greatly reduced. This means that the motor's gearbox can be smaller, lighter, and will show less play and friction losses.

Finally the cam offers also a constructionally simple solution, requiring just a single cam disc with spiraling grooves, rather than individual linkages.

\subsection{Mechatronic Implementation}

This section presents the mechatronic implementation of the SHERPA gripper, including the construction of the fingers and actuation mechanism, as well as the electronics and control of the gripper.

The construction of the gripper made extensive use of rapid prototyping techniques. The components of the fingers and the cam disc are laser cut from polyoxymethylene (POM), while the housing and guiding cone are 3D printed from crylonitrile butadiene styrene (ABS). The gripper has a total weight of $190 \mathrm{~g}$ and external dimensions of $110 \mathrm{~mm} \times 60 \mathrm{~mm}$. 


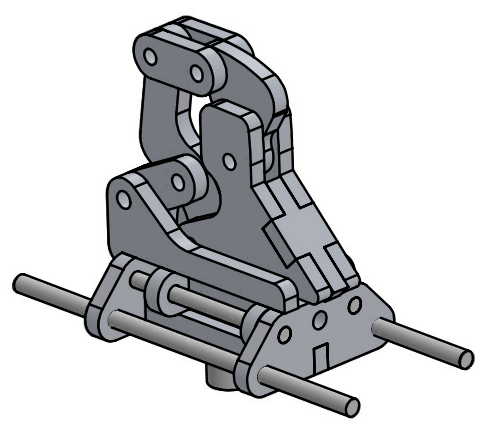

Figure 4.9: The finger design including linear guides for the proximal phalange and the carriage. The joints of the link are simple steel pins.

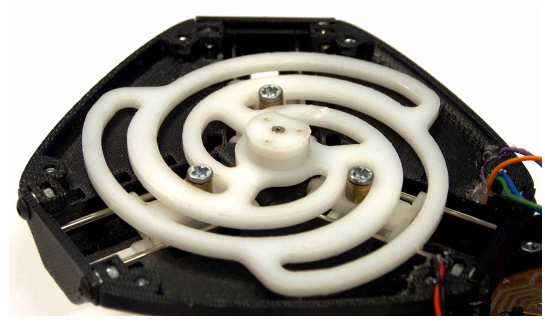

Figure 4.10: The laser cut cam disc with spiraling grooves and rollers, driving the fingers.

\subsubsection{Fingers \& Linear Guide}

The fingers, shown in Figure 4.9, have been assembled of laser cut pieces of POM. This not only represents an easy and effective way to construct such a linkage mechanism, but this low-friction material is also highly suited to incorporate the linear guide mechanisms.

Linear guides are needed to enable the latching mechanism of the fingers, and also to guide the fingers when they are extended towards the interface. These guides are realized with steel rods, that glide in fitted holes in the POM parts. Due to the favorable material pairing, the implemented mechanism provides a good guidance with very low friction and virtually no play.

The spring returning the phalanges of the fingers to their retracted position is integrated between the carriage and the proximal phalange of the fingers.

\subsubsection{Cam Mechanism \& Actuation}

The cam disc, shown in Figure 4.10, has been laser cut out of 3mm thick POM. The profile was executed as three spiraling grooves, that constrain the position of the fingers through rollers running in the grooves, with a pitch that has been matched to the 
actuator performance.

Because of the extremely low actuator requirements, a compact and light motor has been selected, namely a medium-power Pololu micro metal gearmotor with a gear ratio of $150.6: 1$. The motor provides a free run speed of $n=150 \mathrm{rpm}$ and a stall torque of $\tau_{\max }=0.2 \mathrm{Nm}$ at $6 \mathrm{~V}$. The pitch of the cam profile is chosen as $b=20 \mathrm{~mm} / \pi$, which requires a maximum driving angle $\theta_{\max }=180^{\circ}$ to achieve a stroke of $20 \mathrm{~mm}$, according to Equation 4.7 , and results in a theoretical closing speed of $t=\theta_{\max } / n=0.2 \mathrm{~s}^{1}$ at $6 \mathrm{~V}$. According to Equation 4.8 , the gripper can generate a radial force of $F_{r}=\tau_{\max } / b=32 \mathrm{~N}$ at the fingers.

\subsubsection{Electronics \& Control}

The gripper's electronics are integrated into a single PCB that is mounted at the back of the gripper. The gripper is controlled by an Arduino Nano 3.0 with Atmel Atmega 328 processor and can communicate via USB, CAN, SPI, $\mathrm{I}^{2} \mathrm{C}$, or simply through GPIO pins.

The motor is powered with an Allegro Microsystems A4953 full-bridge DMOS PWM motor driver, while the position of the cam disc is measured by an ams AS5048 magnetic rotary encoder.

The power for the Arduino and motor driver is supplied by a Traco Power DC/DC Converter TSR 3-24150, that provides 9V output voltage, from an input range of 9V to $24 \mathrm{~V}$.

A VL6180X proximity sensor, mounted in the dome, measures the distance to the interface, while tactile switches detect a contact with the dome, to aid in the docking process.

\subsection{Experiments}

This section presents the experiments that were carried out to demonstrate the gripper's ability to grasp and securely hold the interface under a load of $2 \mathrm{~kg}$, which corresponds to grasping and holding the UAV. The experimental setup is shown in Figure 4.11. The interface is mounted to a load of $2 \mathrm{~kg}$ via an ATI F/T-Mini40 force-torque sensor to measure the force with which the gripper is holding the interface.

In the experiments the gripper was placed above the interface, and the outwards motion of the fingers activated. A precise alignment of the gripper is not required, as the extending fingers guide the gripper into the correct position and achieve a secure form lock. The shape of the gripper and the interface provide a robust grasp, that is insensitive to small initial misalignments.

Once the gripper was latched onto the interface, it was lifted, so that the gripper held the weight of the load. The gripper was then rotated, to apply also tangential

\footnotetext{
${ }^{1}$ This figure underestimates the closing time by neglecting friction.
} 


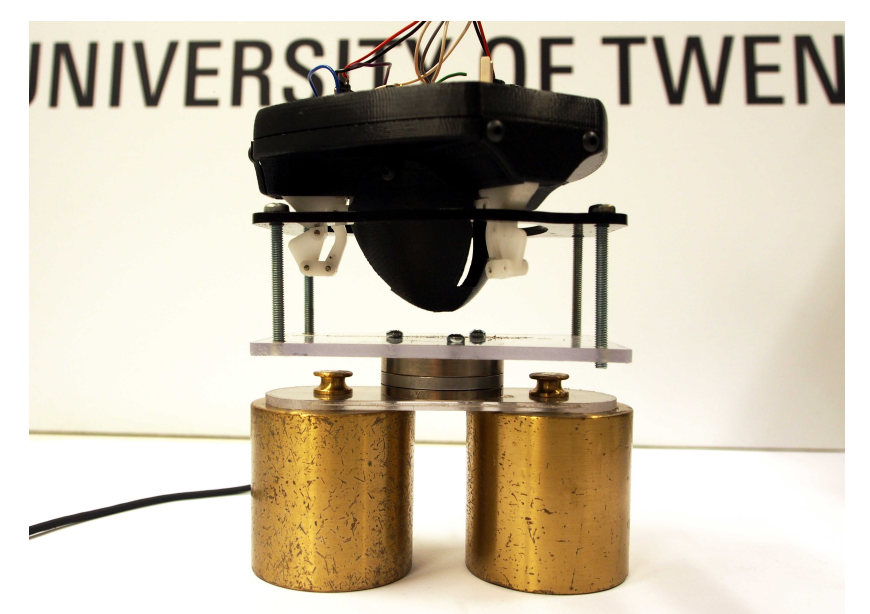

Figure 4.11: The setup used to demonstrate the functionality of the gripper. The gripper engages the interface, which is mounted on a mass of $2 \cdot 1 \mathrm{~kg}$. Between the interface and the load, an ATI F/T-MINI40 force-torque sensor measures the force exerted on the gripper.

forces, and placed back on the ground. The fingers were retracted again, and the interface released.

Figure 4.12 a shows the cam-angle $\theta$ that drives the fingers during the experiments, while Figure $4.12 \mathrm{~b}$ shows the control input to the motor. The actuator is only powered while extending and retracting the fingers, thus it does not contribute to the holding force, even if a load is acting on the gripper. This shows that the gripper indeed achieves a mechanically locked position when it is latched to the interface.

Figure 4.13 shows a detailed view of the cam position and PWM signal during grasping. It can be seen that the gripper latches onto the interface in only $0.5 \mathrm{~s}$.

Figure $4.12 \mathrm{c}$ shows the forces transmitted through the connection between gripper and the interface. The experiments show that the required load of $2 \mathrm{~kg}$ is securely held, even if the force is not applied normal to the interface.

\subsection{Conclusions}

The mechatronic design of the SHERPA gripper, which has been presented and experimentally validated in this chapter, meets the objectives required by $\mathbf{E O 3}$ for robust grasping and manipulation of the UAVs. The latching mechanism of the gripper's fingers not only provide a secure grasp, capable of holding the required load, but in combination with the actuation mechanism, do so by locking the mechanism in a singular position. This greatly reduces the actuator requirements, permitting the use of only a single small motor, which contributes to a compact, light, and fast device. The gripper is furthermore designed in such a way, that its mechanical structure guides it 


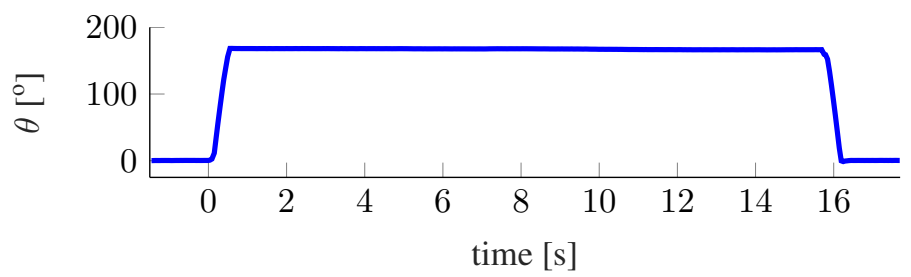

(a)

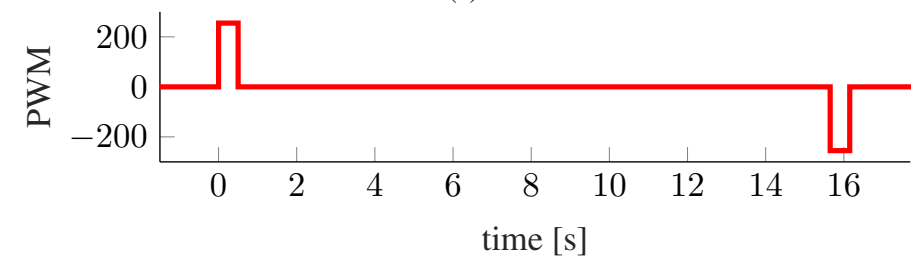

(b)

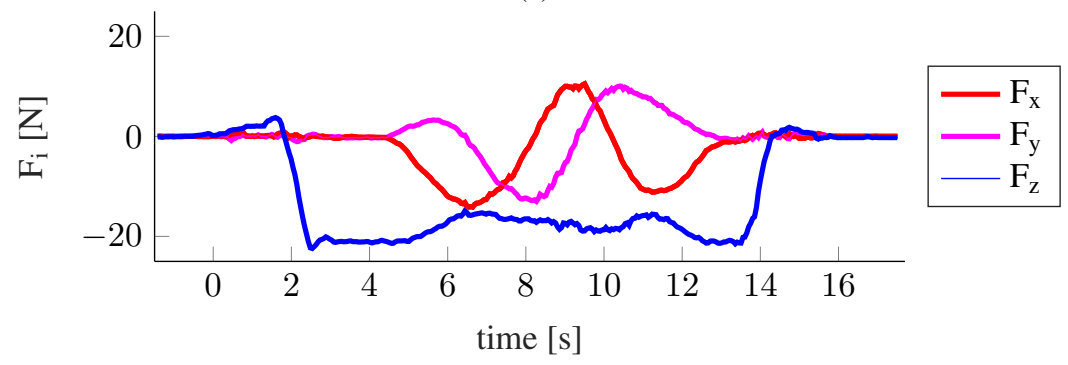

(c)

Figure 4.12: The experimental results demonstrating the grippers ability to latch onto the interface and securely hold the required load. Figure 4.12a shows the cam angle $\theta$, that moves the fingers into the locking position. Figure $4.12 \mathrm{~b}$ shows the control input to the motor driver, while Figure $4.12 \mathrm{c}$ shows the forces $F_{i}$ acting on the gripper. It can be seen that the motor is only active during grasping and releasing. No power is required to generate the holding force of the gripper.

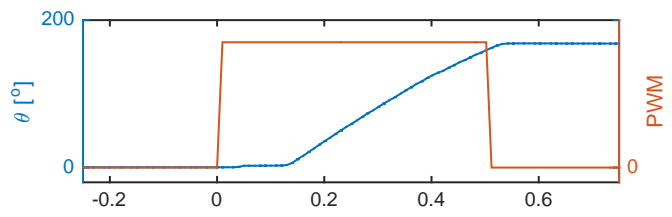

Figure 4.13: Angle $\theta$ of the cam disc and the pwm control signal during grasping. The gripper has latched onto the interface within $0.5 \mathrm{~s}$. The visible latency between sending the motor command and reading the encoder angle do not affect performance, as the fingers are controlled in a feed-forward scheme. 
into the interface, making the grasping operation simple from a control point of view, and robust against misalignments.

The gripper is well suited and ready to begin it's task of manipulating UAVs for the SHERPA project. Moreover its design can easily be applied to other fields. 


\section{CHAPTER 5}

\section{Towards Elastic Control of SEMI-COMPliant MECHANISMS}

Compliant robotic manipulators can in general not achieve arbitrary mechanical Cartesian compliances, even if they are equipped with Variable Stiffness Actuators (VSAs). Their workspace compliance rather also depends on their pose and actuation topology, including the number and placement of compliant joints. After introducing basic concepts of differential geometry and formulating manipulator kinematics based hereon, this chapter investigates in detail how a mechanism's compliant joints affect the constraints on the elastic end effector motions and forces. It provides the tools to answer the research objective $\mathbf{R O 3}$, namely how the actuation topology should be structured, such that a desired workspace compliance can be achieved with a minimal number of VSAs, by analyzing how the workspace is partitioned into complementary spaces of elastic and constraint motions and forces. Furthermore the objective RO4 to formulate manipulation tasks such that the system's passive compliance is properly exploited is addressed by providing projections of the end effector motions and forces onto the spaces of passive compliant twists and wrenches. The control of the mechanical workspace compliance is experimentally validated. 


\subsection{Introduction}

Even though industrial robots have traditionally been designed to be as rigid as possible, physically compliant systems have many advantages for safe interaction and cooperative tasks, as reflected in recent trends in robotics. Manipulators equipped with Series Elastic Actuators (SEAs) [41] and Variable Stiffness (VSAs) [42] benefit from enhanced safety [44, 45], impact absorption [46] and energy storage [37, 52, 53], and also improved force control and peak performance capabilities [47, 48, 49, 50, 51]. These advantages, however, come at the cost of increased mechanical complexity, often leading to larger and heavier systems. Most compliant manipulators will furthermore not be able to achieve arbitrary Cartesian compliances, even when equipped with VSAs, unless they are drastically overactuated compared with traditional robotic manipulators [151, 152]. The symmetric (at equilibrium) compliance matrix $C \in \mathbb{R}^{6 \times 6}$ namely may have $\frac{1}{2} 6(6+1)=21$ independent elements (the diagonal and upper triangular elements), more dofs than are practical for a robotic manipulator. The elastic motions of the end effector are thus in general constrained and depend on the motions and forces allowed by the joints and the manipulator's pose.

Currently mechanically compliant systems are augmented with an active compliance controller, to achieve the desired workspace behavior [151, 153, 154]. However, the goal should be to exploit the mechanical compliance as well as possible in order to retain its benefits for interaction and manipulation tasks. Passivity $[155,156]$ hereby is a key feature to ensure the system's safety and stability, even under unfavorable and unforeseen circumstances, such as communication delays [157], where mechanical compliance can add an additional and intrinsic degree of robustness [158].

Due to the additional complexity, however, it needs to be evaluated if it still is advantageous to add physically compliant actuators at every degree of freedom (dof) of a system, or if a good performance can also be achieved through an actuation topology with fewer compliant joints. A framework for the analysis of the workspace compliance of a serial compliant manipulator is presented here, which allows to investigate in detail how each joint partitions the workspace into compliant and constraint parts, and thus contributes to the system's behavior. A coordinate invariant approach is chosen $[159,160,161]$, based on screw theory and differential geometry $[162,163,164]$. While this formulation is well known, it is used here to obtain an intuitive description of the workspace decomposition, and presents a way to formulate tasks in terms of only compliant forces and motions. This allows to formulate control problems relating to the manipulator's end effector compliance and interaction forces, and to evaluate the manipulator's actuation topology.

The mathematical background needed for the work presented in this chapter is outlined in Section 5.2, after which Section 5.3 introduces the kinematics, compliance tensor, and partitioning of the space of twists and wrenches for a single dof joint, before Section 5.4 extends this framework to serial compliant linkages. A metric which can be used to evaluate the end effector compliance for control problems and for the 
optimization of the actuation topology is presented in Section 5.5, and experimentally validated on the SHERPA arm, a hybrid rigid-compliant serial manipulator presented in this thesis, in Section 5.6, before concluding remarks are drawn in Section 5.7.

\subsection{Mathematical Foundations}

This section provides the mathematical background and introduces important foundations needed in the remainder of this chapter. Using Lie groups theory, the positive isometry group $S E(3)$ of the Euclidean three-dimensional space describes physical rigid body configurations and motions. Twists are defined as elements of the Lie algebra $s e(3)$, while wrenches are elements of the dual algebra, denoted as $s e^{*}(3)$. Screw theory, the second main way to describe rigid body motions, provides a formulation for the geometry of lines, through which every rigid body displacement can be described by a rotation about a line and a translation along the same line. Twists and wrenches, describing the generalized motions of and forces applied to a body, are then associated with screws. While the aim of this section is to provide a self contained presentation of the topic, the reader is referred to $[162,165,166]$ for a thorough treatment of the topic.

\subsubsection{Configuration Space of Compliant Mechanisms}

Each element of the configuration manifold $\mathcal{Q}$ completely specifies the configuration of a mechanical system, and is a minimal "set" of generalized coordinates $q$, such that the number of generalized coordinates (for systems with holonomic constraints) is equal to the number of dofs of the system ${ }^{1}$. Many robotic manipulators have the structure of a serial kinematic linkage, which will be considered here, and which is composed of two extremities, a base and an end effector, which are connected through a chain of connecting links. For a complete description of rigid mechanisms, including forking linkages found for example in robotic hands, or closed kinematic chains, see for instance [165].

As will be introduced in Section 5.2.3, the relative configuration manifold of two bodies connected through a lower pair, a certain type of kinematic pair, corresponds to a subgroup of $S E(3)$ [165]. If the $n+1$ links of the kinematic chain are furthermore connected through $n$ one-dimensional basic joints ${ }^{2}$ then the configuration of the system can be locally uniquely described by the vector $q=\left(q_{1}, q_{2}, \ldots, q_{n}\right)^{T}$ of $n$ scalar generalized coordinates.

When, however, the joints are compliant, elastic deflections arise as additional dofs and need to be included in the configuration space. This becomes apparent when

\footnotetext{
${ }^{1}$ Generalized coordinates are not necessarily minimal, this however avoids additional constraints and allows an explicit description of the system.

${ }^{2}$ Three types of basic joints, one degree of freedom kinematic-pairs, exist, which permit rotational, translational, or screw motions between a pair of rigid bodies.
} 


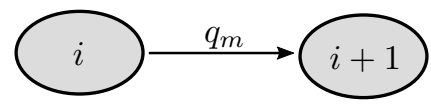

(a) Two rigid bodies connected through a basic joint, which relative position is described by the generalized coordinate $q$

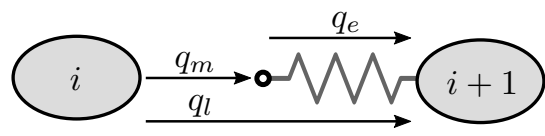

(b) Two elastically linked rigid bodies. Their relative configuration $q_{l}$ depends on the actuated motor position $q_{m}$, and the configuration of the elastic element $q_{e}$, such that a vector of generalized coordinates is $q=\left(\begin{array}{ll}q_{m} & q_{e}\end{array}\right)^{T}$.

Figure 5.1: Two rigid bodies connected with a basic joint, that are actuated rigidly (Figure 5.1a) or compliantly (Figure 5.1b), illustrating the resulting configuration manifolds.

considering the simple one-dimensional example shown in Figure 5.1a, where the relative position of two bodies, connected through the relative configuration manifold $\mathcal{Q}_{i+1}^{i}$, is described by the generalized coordinate $q$, with $\operatorname{dim} \mathcal{Q}_{i+1}^{i}=1$.

When the two bodies are connected through a compliant coupling as in Figure 5.1b, their relative configuration is described by the actuated motor position $q_{m} \in \mathcal{Q}_{i+1, m}^{i}$, and the position of the unactuated elastic element $q_{e} \in \mathcal{Q}_{i+1, e}^{i}$, such that the vector of generalized coordinates describing the joint is $q=\left(\begin{array}{c}q_{m} \\ q_{e}\end{array}\right) \in \mathcal{Q}_{i+1}^{i}$. The link-side joint position is then given by $q_{l}=q_{m}+q_{e}$. The relative configuration manifold $\mathcal{Q}_{i+1}^{i}$ of the elastically coupled bodies is thus composed of the actuated and elastic configuration manifolds $\mathcal{Q}_{i+1}^{i}=\mathcal{Q}_{i+1, m}^{i} \times \mathcal{Q}_{i+1, e}^{i}$, where $\times$ is the Cartesian product, and the dimension of the configuration of the basic compliant joint is $\operatorname{dim} \mathcal{Q}_{i+1}^{i}=2$.

In the absence of additional constraints, a total configuration manifold $\mathcal{Q}$ for a serial kinematic chain of $n+1$ links can simply be constructed as the Cartesian product of the $n$ relative configuration manifolds $\mathcal{Q}_{i+1}^{i}$ connecting the neighboring links, which include the compliant deflections:

$$
\mathcal{Q}=\mathcal{Q}_{1}^{0} \times \mathcal{Q}_{2}^{1} \times \ldots \times \mathcal{Q}_{n}^{n-1}
$$

It follows, that the total number of dofs of such a system is $\sum_{i=1}^{n} \operatorname{dim} \mathcal{Q}_{i+1}^{i}$.

\subsubsection{Manipulator Workspace}

Rigid-body motions in three-dimensional Euclidean space can be described with the set of relative homogeneous matrices $H$, such that the relative configuration of a body, i.e. its position and orientation with respect to a reference, is identified as an element of the positive isometry group $S E(3)$. The aim of this section is to introduce this group, along with the associated Lie (co-) algebra elements twists and wrenches, which respectively describe the motion and the set of forces acting on the manipulator's end effector, in a compact way. For a complete treatment of the framework 


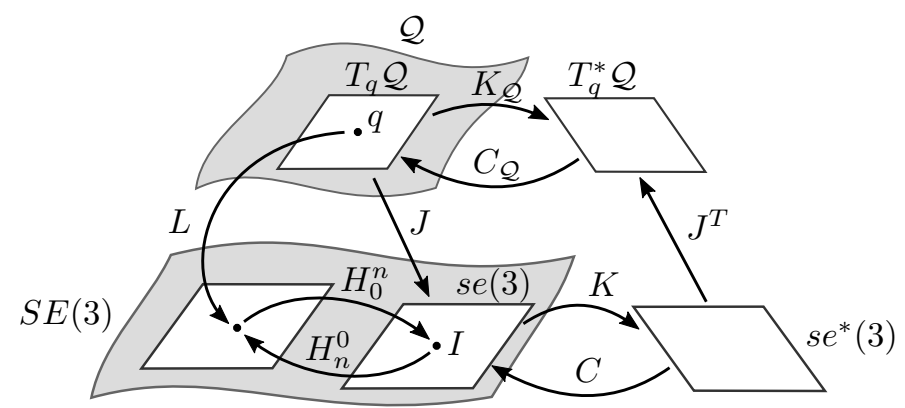

Figure 5.2: The commutation diagram of the configuration and workspace manifolds $\mathcal{Q}$ and $\mathcal{Y}:=S E(3)$ with their velocity and force tangent and co-tangent spaces. The generalized end effector velocities $\dot{H} \in$ $T_{y} S E(3)$ are described as twists, which are members of the Lie algebra $s e(3)$, defined at the identity element of the Lie group $S E(3)$. The velocities are thus transformed through multiplication with $H^{-1}$, such that $\tilde{T}:=\dot{H} H^{-1}$.

for defining motion between objects using screw theory and Lie groups, the reader is referred to [165].

The six-dimensional pose of an object can be described by a three-dimensional translation for the position, and a rotation describing the object's orientation, both with respect to a reference. The group of translations in three dimensions $T(3)$ is homeomorphic to the vector space $\mathbb{R}^{3 \times 1}$ with vector addition as binary operation. In this work, an object's orientation is represented through a rotation matrix $R \in$ $S O$ (3) such that the relative pose in the workspace is represented with a translation and rotation through a homogeneous matrix $H \in S E(3)$

$$
S E(3)=\left\{H=\left(\begin{array}{cc}
R & p \\
0_{1 \times 3} & 1
\end{array}\right) \mid R \in S O(3), p \in \mathbb{R}^{3}\right\}
$$

where $R \in S O(3)$ is a $3 \times 3$ rotation matrix such that $R^{T}=R^{-1}$ and $\operatorname{det} R=1$, and $p \in \mathbb{R}^{3}$ a translation.

Now that the relative configuration of a rigid body can be expressed as a homogeneous matrix, the motions, or changes in the configuration, can be considered. If a matrix $H(t) \in S E(3)$ is a differentiable function of time then the product $\dot{H} H^{-1}$ belongs to the Lie algebra $s e(3)^{3}$, the tangent space at the identity element of the Lie group $S E(3)$ :

$$
\tilde{T}:=\dot{H} H^{-1} \in \operatorname{se}(3)
$$

as illustrated in Figure 5.2.

The elements $\tilde{T} \in \operatorname{se}(3)$ of the Lie algebra are the differential geometric representation of twists, and express configuration independent velocities for rigid bodies.

\footnotetext{
${ }^{3}$ The product $H^{-1} \dot{H}$ also belongs to the algebra, owing to the possibility of left and right invariant transport [167]
} 
Twists can be represented in matrix form as $\tilde{T} \in \mathbb{R}^{4 \times 4}$, or as the vector $T \in \mathbb{R}^{6}$ of the Plücker coordinates of $\tilde{T}$, using the notation

$$
\tilde{T}=\left(\begin{array}{cc}
\tilde{\omega} & v \\
0_{1 \times 3} & 0
\end{array}\right) \Rightarrow T=\left(\begin{array}{c}
\omega \\
v
\end{array}\right)
$$

where $\tilde{\omega} \in \mathbb{R}^{3 \times 3}$ represents the skew-symmetric angular velocity, $\omega \in \mathbb{R}^{3}$ the vector of angular velocities, and $v \in \mathbb{R}^{3}$ the velocity of a specific point.

Since twists are elements of the Lie algebra $s e(3)$ representing a generalized motion, there exist elements of the dual space, the co-tangent space of $S E(3)$ in the identity, denoted as $s e^{*}(3)$, representing, among other, generalized forces between objects. Like twists, these wrenches can be written in matrix and co-vector form

$$
\tilde{W}=\left(\begin{array}{cc}
\tilde{f} & \tau^{T} \\
0_{1 \times 3} & 0
\end{array}\right), \quad W=\left(\begin{array}{ll}
\tau & f
\end{array}\right)
$$

where $\tau$ represents the torque, $\tilde{f}$ the skew-symmetric matrix of forces, and $f$ the linear force.

A twist describes the relative geometric motion between two objects $i$ and $j$. In the following twists (along with homogeneous matrices and wrenches) expressed in the observer frame $\Psi_{0}$ will only be denoted by these two indices $i$ and $j$ of the associated bodies as $T_{i}^{j}$ or $T_{j}^{i}$. However, they can also be expressed in a different reference frame, for instance attached to one of the frames $i$ or $j$. In this case they can be transformed between those references with the Adjoint representation of the homogeneous matrix $H_{i}^{j}$ describing the relative configuration of the frames, expressed in the body-fixed frame $\Psi_{j}$ :

$$
T_{i}^{j, j}=A d_{H_{i}^{j}} T_{i}^{i, j}, \quad A d_{H_{i}^{j}}:=\left(\begin{array}{cc}
R_{i}^{j} & 0 \\
\tilde{p}_{i}^{j} R_{i}^{j} & R_{i}^{j}
\end{array}\right)
$$

where $T_{a}^{i, b}$ refers to the motion of body $a$ with respect to body $b$ expressed in the reference frame $\Psi_{i}$.

Since wrenches are the dual of twists, operations for twists, like the adjoint mapping for coordinate changes, can similarly be applied to wrenches after transposition.

\subsubsection{Manipulator Kinematics}

Section 5.2.2 introduced homogeneous matrices $H \in S E(3)$ that characterize relative configurations, and twists $T \in s e(3)$ that describe relative motions. This framework can be combined with the notion of generalized coordinates presented in Section 5.2.1, to derive the forward kinematic mapping $L(q): \mathcal{Q} \rightarrow S E(3)$ between the configuration manifold $\mathcal{Q}$ and the workspace $S E(3)$, as well as a representation of the differential mapping $L_{*}(q)$ between their tangent and co-tangent spaces as illustrated in Figure 5.3. 


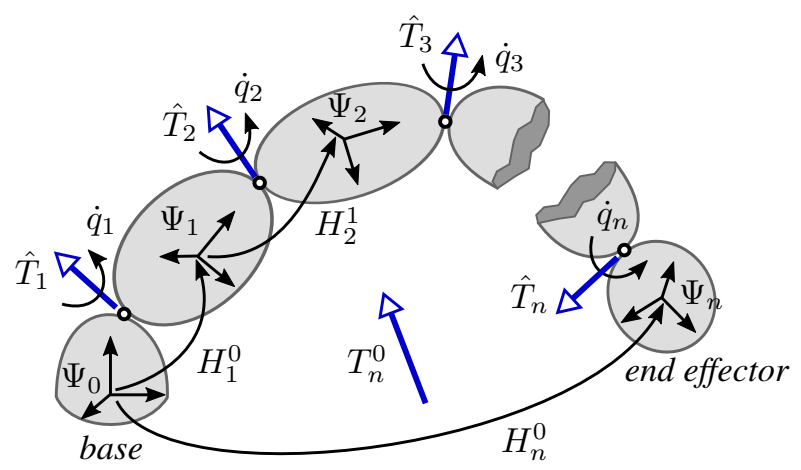

Figure 5.3: A kinematic chain of rigid bodies, connecting the base with the end effector, the final link of the chain. A local coordinate frame $\Psi_{i}$ is associated with each body in the chain, while the frame $\Psi_{0}$ is chosen as the inertial reference, or observer frame. Neighboring bodies are connected through kinematic pairs that constrain the relative motion between them. The relative configuration of the body frame $\Psi_{i}$ with respect to body frame $\Psi_{i-1}$ is described through the homogeneous matrix $H_{i}^{i-1}$, while their relative motion is described by a unit twist $\hat{T}_{i}$ along the unconstrained dof, and the corresponding generalized joint velocity $\dot{q}_{i}$. The end effector twist, with respect to the reference frame $\Psi_{0}, T_{n}^{0}$ is equal to the sum of the twists of the kinematic pairs.

The relative configuration of two bodies connected through a lower pair is spanned by the exponential of an element of the Lie algebra $s e(3)$. Such joints can be defined by unit twists [162] of the form

$$
\hat{T}=\left(\begin{array}{l}
\hat{\omega} \\
\bullet
\end{array}\right) \quad \text { or } \quad \hat{T}=\left(\begin{array}{l}
0 \\
\hat{v}
\end{array}\right)
$$

where $\hat{\omega}$ and $\hat{v}$ are unit vectors. The relative motion of these bodies is constrained along a unique twist

$$
T_{(i+1)}^{j, i}=\alpha \hat{T}_{(i+1)}^{j, i}, \quad \alpha \in \mathbb{R}
$$

where $\hat{T}_{(i+1)}^{j, i}$ is a constant unit twist if its reference frame $\Psi_{j}$ is fixed to either body $i$ or $i+1$. This relative twist can be used to describe the relative motion between the bodies with the joint velocity $\alpha=\dot{q}_{i}$, or a configuration change with the generalized coordinate $\alpha=q_{i}$, and using exponential coordinates.

The relative configuration between two bodies connected through joint $i$ can be expressed as a homogeneous matrix with the matrix exponential map $e: \operatorname{se}(3) \rightarrow$ $S E(3) ; \quad \hat{\tilde{T}} \mapsto e^{\hat{T}}$ of the unit twist and generalized coordinate $q_{i}$ that describes the relative motion of the joint:

$$
H_{i}^{(i-1)}\left(q_{i}\right)=e^{\hat{\hat{T}}_{i}^{(i-1),(i-1)} q_{i}} H_{i}^{(i-1)}(0)
$$

where $H_{i}^{(i-1)}(0)$ is the configuration for $q_{i}=0$. With this, Brockett's exponential 


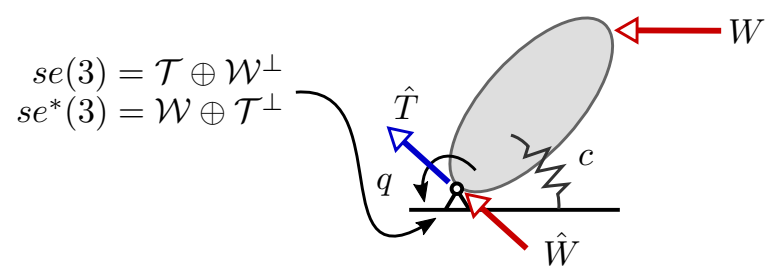

Figure 5.4: A basic compliant joint defines a body's kinematics through a unit twist $\hat{T}$, and a geometrically aligned unit wrench $\hat{W}$. The relative configuration of the body can be described through the generalized coordinate $q$, which describes the joint's elastic displacement, and generates an elastic wrench $\hat{W} \delta q / c$, which acts along the same line as $\hat{T}$. The space of generalized motions of the body, the Lie algebra $s e(3)$, is the direct sum of the set of elastic twists $\mathcal{T}$ along $\hat{T}$, and the set of twists $\mathcal{W}^{\perp}$ dual to $\hat{W}$. Analogously, the space of generalized forces is the direct sum of wrenches $\mathcal{W}$ along $\hat{W}$, and the set of constraint wrenches dual to $\hat{T}$, which are balanced by the kinematic pair.

formula for the direct kinematic map $L: \mathcal{Q} \rightarrow S E(3)$ can be derived as [168]:

$$
H_{n}^{0}(q)=e^{\tilde{\hat{T}}_{1}^{0,0} q_{1}} e^{\tilde{\hat{T}}_{2}^{0,1} q_{2}} \ldots e^{\tilde{\hat{T}}_{n}^{0, n-1} q_{n}} H_{n}^{0}(0)
$$

The geometric manipulator Jacobian $J(q): T \mathcal{Q} \rightarrow s e(3)$ expresses how each relative twist between two links contributes to the end effector twist, and expresses these in terms of the joint velocities $\dot{q}$ according to Equation 5.8:

$$
T_{n}^{0}=\left(\begin{array}{llll}
\hat{T}_{1} & \hat{T}_{2} & \ldots & \hat{T}_{n}
\end{array}\right) \dot{q}=J(q) \dot{q}
$$

where the columns of $J(q)$ are the unit twists $\hat{T}_{i}=A d_{H_{i+1}^{0}} \hat{T}_{i}^{(i+1),(i+1)} \in \operatorname{se}(3)$ expressed in the observer frame $\Psi_{0}$, and the vector of the generalized joint velocities $\dot{q}:=\frac{\partial}{\partial t}\left(q_{1}, \ldots, q_{n}\right)^{T}$.

As the manipulator Jacobian $J(q)$ maps the configuration velocity $\dot{q} \in T_{q} \mathcal{Q}$ to a twist in the workspace, the transposed map $J^{T}(q)$ maps wrenches $\in s e^{*}(3)$ in the workspace to configuration space torques or forces.

\subsection{The Basic Compliant Joint}

To illustrate the set of elastic motions and forces a given manipulator can achieve, it is instructive to first consider the one-dimensional case of a rigid body with a basic compliant joint. The elastic twists and wrenches defined by the joint, and the partitioning of the workspace are presented here, while the compliant serial mechanism is treated in the following section.

Twists and wrenches, have been viewed as vectors and co-vectors up to this point. They are, however, tensors $[169,170]$, and are explicitly treated as such in this section, with Einstein notation being used, unless stated otherwise. 


\subsubsection{The Compliant Kinematic Pair}

Consider a one-dimensional basic joint, as described in Equation (5.8), constraining the motion of a rigid body through a unit twist $\hat{T}^{q} \in s e(3)$ and with the generalized coordinate $q \in \mathbb{R}$, as illustrated in Figure 5.4, such that an infinitesimal displacement of the body can be described as

$$
\delta T^{q}=\hat{T}^{q} \delta q
$$

The component of any wrench $W_{p} \in s e^{*}(3)$ in the "direction" of unit twist $\hat{T}^{p}$ can be calculated with

$$
\hat{T}^{p} W_{p}=\tau
$$

The scalar generalized joint force $\tau \in \mathbb{R}$ hereby represents that part (coefficient) of the wrench $W_{p}$, which acts along the unit wrench $\hat{W}_{l}$, geometrically aligned with $\hat{T}^{l}$. When multiplied with $\hat{W}_{l}$, it generates the elastic wrench $W_{l}=\hat{W}_{l} \tau$ acting in the direction of the joint. If the joint has a finite compliance $c$, it causes a deflection $\delta q$, that is written as

$$
\delta q=c \tau=c \hat{T}^{l} W_{l}
$$

Equation 5.12 can now be written in the following form

$$
\delta T^{q}=\hat{T}^{q} c \hat{T}^{l} W_{l}
$$

where the operator $\hat{T}^{q} c \hat{T}^{l}$ acting on the wrench $W_{l}$ is the symmetric $(2,0)$-tensor

$$
C^{q l}:=c \hat{T}^{q} \otimes \hat{T}^{l}
$$

obtained as the tensor product of the unit twists, such that Equation (5.15) can finally be written as

$$
\delta T^{q}=C^{q l} W_{l}
$$

It can be seen that $C^{q l}$ is the compliance tensor, which links the space of generalized end effector forces to that of generalized displacements. This is also the reason for using small displacements $\delta T^{q}$ and $\delta q$, as the compliance is only characterized by a tensor in the equilibrium position, and may become asymmetric when a mechanism is not in equilibrium [171]. Please note that this tensor has rank $=1$.

\subsubsection{Dual Complements and Workspace Decomposition}

Twist and wrenches are dual, and result in power when paired. This power is defined as the duality product $P=\left\langle W_{l} \mid T^{l}\right\rangle$ and here refers to the power transferred in the elastic direction of the basic compliant joint:

$$
P=\left\langle W_{l} \mid T^{l}\right\rangle=W_{l} T^{l}=\frac{\delta q}{c} \hat{W}_{l} \hat{T}^{l} \dot{q}=\tau \dot{q}
$$



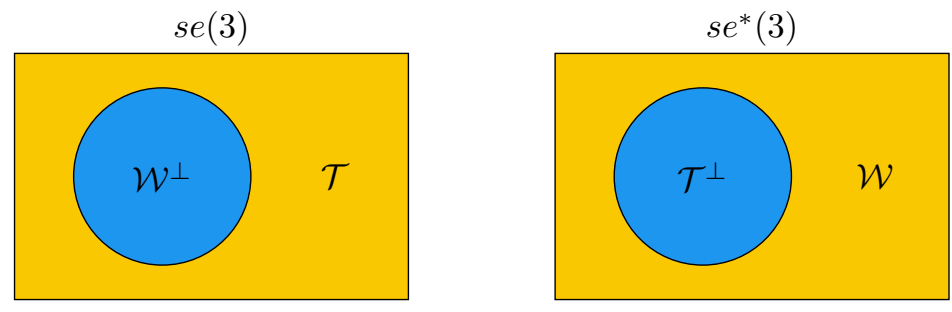

Figure 5.5: Venn diagrams showing how a basic joint partitions the spaces of generalized motions and forces $s e(3)$ and $s e^{*}(3)$ applied to a body into two real subsets, the five-dimensional spaces of constraint twists and wrenches $\mathcal{W}^{\perp}$ and $\mathcal{T}^{\perp}:=\operatorname{ker} C^{q l}$, and the one-dimensional space of elastic twists and wrenches $\mathcal{T}:=\operatorname{span} \hat{T}^{q}$ and $\mathcal{W}:=\operatorname{span} \hat{W}_{q}$ that are geometrically aligned with the unit twist $\hat{T}^{q}$ of the joint.

Since $\hat{W}_{l} \hat{T}^{l}=1$, the potential energy stored in the elastic element connected to the joint is consequently $V(\delta q)=\int P d t=\int \frac{\delta q}{c} \dot{q} d t=\int \frac{1}{c} \delta q d q=\frac{1}{2} \frac{\delta q^{2}}{c}$. It can be seen here, that potential energy storage in the mechanism is a consequence of the deflection $\delta q$ of a compliant joint, and thus associated with the configuration space $\mathcal{Q}$ presented in Section 5.2.1.

With this definition of the duality product, the set of the joint's constraint wrenches $\mathcal{T}^{\perp} \subset s e^{*}(3)$ can be defined. The operator $\perp$ hereby denotes a subspace's dual complement, which is the set of all elements of its dual space, for which the duality product of every element of the original subspace is zero (results in no power). The set of wrenches $\mathcal{T}^{\perp}$ are the generalized forces acting on the body, which do not cause an elastic displacement $\delta T^{q}$ along the unit twist $\hat{T}^{q}$ defining the body's elastic motion, and which do not perform work on a twist $T^{q} \in \mathcal{T}:=\operatorname{span} \hat{T}^{q}$ :

$$
\mathcal{T}^{\perp}=\left\{W_{q} \in \operatorname{se}^{*}(3) \mid\left\langle W_{q} \mid T^{q}\right\rangle=0, T^{q} \in \mathcal{T}\right\}
$$

In the same manner the set $\mathcal{W}^{\perp} \subset \operatorname{se}(3)$ of constrained twists can be defined, on which a wrench $W_{q} \in \mathcal{W}:=$ span $\hat{W}_{q}$, geometrically aligned with the joint's unit twist $\hat{T}^{q}$, performs no work:

$$
\mathcal{W}^{\perp}=\left\{T^{q} \in \operatorname{se}(3) \mid\left\langle W_{q} \mid T^{q}\right\rangle=0, W_{q} \in \mathcal{W}\right\}
$$

A compliant joint thus partitions the spaces of generalized motions and forces into two complementary subspaces of constrained and elastic twists and wrenches, as illustrated in Figure 5.5, such that any wrench can be written as linear combination of elements of the constraint wrenches $\mathcal{T}^{\perp}$ and a wrench $W_{l} \in \mathcal{W}$ that is aligned with $\hat{W}_{l}$. The corresponding holds for twists, such that we obtain the following direct sums

$$
\begin{aligned}
s e(3) & =\mathcal{T} \oplus \mathcal{W}^{\perp} \\
s e^{*}(3) & =\mathcal{W} \oplus \mathcal{T}^{\perp}
\end{aligned}
$$

Because a basic joint permits motion along one dof, the dimension of the subspaces of elastic twists and wrenches is one. It follows that the dimension of their 


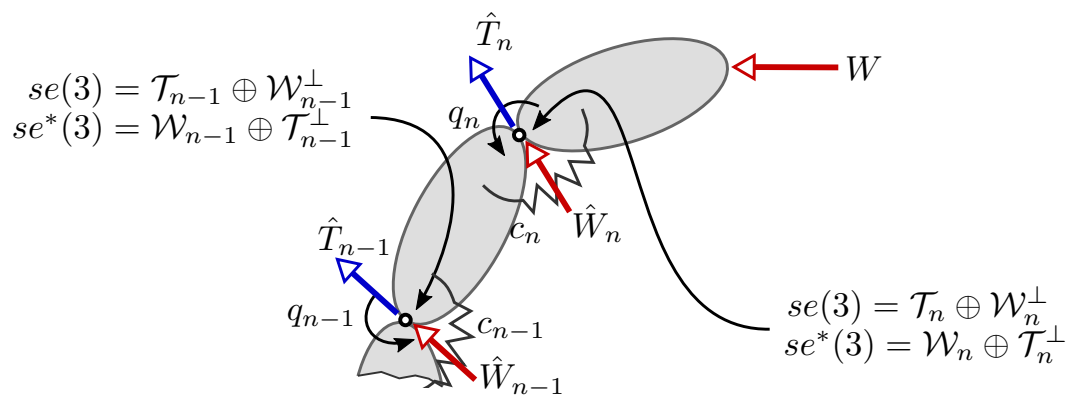

Figure 5.6: A serial kinematic linkage, in which basic compliant joints connect a chain of rigid bodies. Like in the one-dimensional case presented in Figure 5.4, each compliant joint partitions the six-dimensional spaces of generalized end effector forces $s e^{*}(3)$ and displacements $s e(3)$ into a five-dimensional constrained and a one-dimensional elastic subspace.

complementary subspaces must be $6-1=5$ in order to cover all generalized motions and forces in three-dimensional space:

$$
\begin{aligned}
& \operatorname{dim} \mathcal{T}+\operatorname{dim} \mathcal{W}^{\perp}=\operatorname{dim} s e(3) \\
& \underbrace{\operatorname{dim} \mathcal{W}}_{1}+\underbrace{\operatorname{dim} \mathcal{T}^{\perp}}_{5}=\underbrace{\operatorname{dim} s e^{*}(3)}_{6}
\end{aligned}
$$

While the spaces of twists and wrenches can be partitioned by taking the dual complements of the elastic twists and wrenches $\mathcal{T}$ and $\mathcal{W}$, the compliance tensor (5.16) also contains the information about which dofs are constrained (zero compliance), and which are elastic (finite compliance). The kernel of the linear map $C^{q l}: s e^{*}(3) \mapsto s e(3)$ defined in Equation 5.17, thus also defines the set of constraint wrenches that do not cause a displacement of the body. $\mathcal{T}^{\perp}$ can be defined as

$$
\mathcal{T}^{\perp}:=\operatorname{ker} C^{q l} \subset s e^{*}(3)
$$

while the elastic wrenches $\mathcal{W}$ are spanned by the unit wrench $\hat{W}_{l}$, that is geometrically aligned with the unit twist $\hat{T}^{l}$ defining the joint, such that

$$
\mathcal{W}:=\operatorname{span} \hat{W}_{l} \subset s e^{*}(3)
$$

Furthermore the complementary subspaces $\mathcal{T} \subset s e(3)$ and $\mathcal{W}^{\perp} \subset s e(3)$, and $\mathcal{W} \subset s e^{*}(3)$ and $\mathcal{T}^{\perp} \subset s e^{*}(3)$ define projections along which twists and wrenches applied to the body can be decomposed into compliant and constrained motions and forces.

\subsection{The Semi-Compliant Serial Mechanism}

After seeing how a single compliant joint decomposes the spaces of generalized motions and forces $s e(3)$ and $s e^{*}(3)$ into real subsets of compliant and constrained twists 
and wrenches, the effects of connecting a chain of rigid bodies through such basic joints are investigated.

\subsubsection{Manipulator Kinematics and Total Compliance}

Consider a serial mechanism with $n$ dofs indexed from 1 (connected to the base) to $n$ (connected to the end effector), as shown in Figure 5.6, of which $m \leq n$ are elastic joints indexed by a set $E \in 2^{[1 \ldots n]}$, such that the $i$ th joint is compliant if $i \in E$, and $E$ therefore has cardinality $m .2^{[1 \ldots n]}$ hereby refers to the power set of the joints, such that $E$ is that subset of all joints, which contains the compliant joints.

Using the geometric Jacobian defined in Equation (5.11), the infinitesimal displacement of the end effector, the $(n+1)$ th body of the serial chain, can be expressed through the joints' unit twists and displacements $\delta q$

$$
\delta T=J(q) \delta q
$$

where $J(q) \in \mathbb{R}^{6 \times n}$ and the generalized joint displacements $\delta q \in \mathbb{R}^{n}$. Suppose that all not compliant dofs are locked, which is for instance the case for non-backdrivable motors. Then the rigidly actuated joints $j \notin E$ are fully constrained, and the compliant joints $i \in E$ can only move by deflecting their elastic elements, such that they behave as described in the previous Section 5.3.

A new Jacobian $J_{e} \in \mathbb{R}^{6 \times m}$ can be defined, which columns are the $m$ unit twists $\hat{T}_{i}(q)$ of the elastic joints $i \in E$, along with the infinitesimal elastic joint displacements $\delta q_{e} \in \mathbb{R}^{m}$. Note that the indices $e$ and $i$ refer to the newly defined elastic Jacobian and joint deflections, denoted by $e$, and the $i$ th compliant joint, and are not tensor indices. Then

$$
\delta T=J_{e}(q) \delta q_{e}
$$

represents only those end effector motions that are caused by elastic deflections. With the scalar compliance $c_{i}$ of joint $i$ the end effector displacement can be written

$$
\delta T=J_{e}^{T}(q) C_{\mathcal{Q}} J_{e}(q) W_{l}
$$

where $C_{\mathcal{Q}} \in \mathbb{R}^{m \times m}$ is a diagonal matrix of the joint compliances $c_{i}$. Similar to the case for the single joint, the previous Equation (5.29) can be written as

$$
\delta T=\sum_{i=1}^{m} C_{i}(q) W_{l}=C^{q l} W_{l}
$$

where $C_{i}(q):=c_{i} \hat{T}^{i}(q) \otimes \hat{T}^{i}(q)$ is the compliance tensor of each joint $i$, according to Equation (5.16), and $C^{q l}$ is the compliance of the whole mechanism, obtained by summation of the joint compliance tensors (compliance addition for serial structures). 

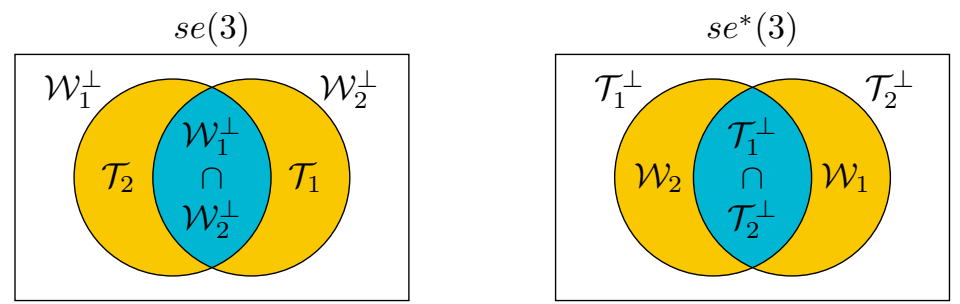

Figure 5.7: Venn diagrams showing how the spaces of generalized motions and forces $s e(3)$ and $s e^{*}(3)$ respectively are partitioned by two independent compliant joints. Each joint spans a one dimensional space of geometrically aligned compliant twists and wrenches $\mathcal{T}_{i}$ and $\mathcal{W}_{i}$, such that the remaining space of constrained motions and forces is four dimensional. As the joints are independent, the elastic subspaces of one joint lie in the constrained space of the other.

\subsubsection{Workspace Decomposition of Serial Mechanisms}

The space of constraint wrenches $\mathcal{T}^{\perp} \subset s e^{*}(3)$ that rigidly constrain the end effector motion through the mechanism's mechanical structure can be defined as the kernel $\operatorname{ker} C^{q l}$ of the total compliance of the mechanism.

$$
\mathcal{T}^{\perp}:=\operatorname{ker} C^{q l}
$$

It is, however, also the intersection of the kernels of the compliance tensors $\mathcal{T}_{i}^{\perp}(q)=\operatorname{ker} C_{i}(q)$ of each compliant joint ${ }^{4}$ :

$$
\mathcal{T}^{\perp}:=\bigcap_{i=0}^{m} \mathcal{T}_{i}^{\perp}(q)
$$

as each joint partitions the workspace into elastic and constrained subspaces.

Furthermore, $s e^{*}(3)$ can be decomposed into the following direct sum:

$$
s e^{*}(3)=\mathcal{W} \oplus_{l \in 2^{[1 \ldots m]}} \mathcal{T}_{l}^{\perp}
$$

where $\mathcal{W}$ is the set of elastic wrenches which are geometrically aligned with all elastic joints, such that $\mathcal{W} \cap \mathcal{T}_{i}^{\perp}=\emptyset \quad \forall i \in E$, and $\oplus_{l \in 2^{[1 \ldots m]}} \mathcal{T}_{l}^{\perp}$ indicates all possible intersections of the kernels $\mathcal{T}_{i}^{\perp}$ which decompose the rest of $s e^{*}(3)$ and which have no intersection among each other.

More detailed insights into each joint's effect on the end effector constraints can be gained by decomposing $s e^{*}(3)$ through such iterative intersections, as shown in the following. Recall Figure 5.5, which shows the decomposition of $s e^{*}(3)$ into two composite elastic and constrained subspaces, $\mathcal{W}_{1}$ and $\mathcal{T}_{1}^{\perp}$ respectively. There now

\footnotetext{
${ }^{4}$ In fact $\mathcal{T}^{\perp}$ is the intersection of the kernels of all $n$ joints, including the rigid joints $j \notin E$. The constraint forces of the rigid joints, however, span the whole $s e^{*}(3)$, such that they do not partition $s e^{*}(3)$ into real subsets, and do not release any additional dofs.
} 
are two possibilities when adding a second basic elastic joint, thus forming a serial compliant mechanism. If both joints are described by the same unit twist, then they are not independent, and leave the same space of wrenches unconstrained. The kernels $\mathcal{T}_{i}{ }^{\perp}$ of their compliance tensors then consequently describe the same space, and the partition of $s e^{*}(3)$ does not change with respect to Figure 5.5.

If the joints, however, are independent, then $s e^{*}(3)$ is decomposed according to Figure 5.7: both kernels $\mathcal{T}_{1}^{\perp}$ and $\mathcal{T}_{2}^{\perp}$ are subsets of $s e^{*}(3)$ and share the intersection $\mathcal{T}_{1}^{\perp} \cap \mathcal{T}_{2}^{\perp}$, which represents the constraint wrenches of the whole mechanism. Since $\operatorname{dim} \mathcal{T}_{i}^{\perp}=5$, and the joints are independent, the space of one joint's elastic wrenches $\mathcal{W}_{i}$ must lie in the other joint's kernel $\mathcal{T}_{j}^{\perp}$, and vice versa. It also follows from the joint's independence that $\mathcal{W}=\emptyset$, as there can be no elastic wrench geometrically aligned with both joints. The next intuitive fact is that $\operatorname{dim}\left(\mathcal{T}_{1}^{\perp} \cap \mathcal{T}_{2}^{\perp}\right)=$ $\operatorname{dim}\left(s e^{*}(3)\right)-\operatorname{dim}\left(\mathcal{W}_{1} \cup \mathcal{W}_{2}\right)=4$, which means that the space of constraint wrenches drops rank for each independent dof that is added to the mechanism.

This process of adding independent joints can be repeated, until six independent dofs have been reached. Each time the space of constraint wrenches $\mathcal{T}^{\perp}=\cap_{i=0}^{m} \mathcal{T}_{i}^{\perp}$ drops rank, and a different one-dimensional space of elastic wrenches $\mathcal{W}_{i}$ is obtained. When the serial mechanism consists of six independent joints, then $\operatorname{dim} \mathcal{T}^{\perp}=\operatorname{dim}\left(s e^{*}(3)\right)-6=0$, and no more independent joints can be found. Figure 5.8 illustrates this with Venn diagrams showing the decompositions of $s e^{*}(3)$ for three to six independent basic elastic joints.

\subsubsection{Compliance Transformation}

While the approach of obtaining the total workspace compliance of a serial compliant mechanism by summing the joint compliance tensors obtained from the unit twists that was presented in this section offers more insight into each joint's contribution, an equivalent, less insightful method exists. The end-effector compliance of a robotic manipulator that is felt in the Cartesian workspace depends on its link and joint compliance. In the majority of modern serial robots the link compliance is sufficiently large, such that the system's compliance depends on that of the joints. In this case, the joint compliance can be transformed into the end effector compliance through the geometric Jacobian map $J(q)$, introduced in Equation (5.11).

Increasingly, robotic manipulators are equipped with intentionally compliant joints to implement desirable properties. In such a case, it is sufficient to consider only the compliant joint's $i \in E$ contribution to the end effector compliance through the elastic Jacobian $J_{e}(q)$ of reduced dimensionality introduced in Equation (5.28), and the diagonal matrix $C_{Q}$ of joint compliances $c_{i}$, through

$$
C:=J_{e}(q) C_{\mathcal{Q}} J_{e}^{T}(q)
$$

as already anticipated in Equation (5.29). In this case the rigid joints can be assumed to be fully constrained, with a degenerate relative configuration manifold of $\operatorname{dim} \mathcal{Q}_{i+1}^{i}=$ 0 , and no contribution to the system's compliance. 

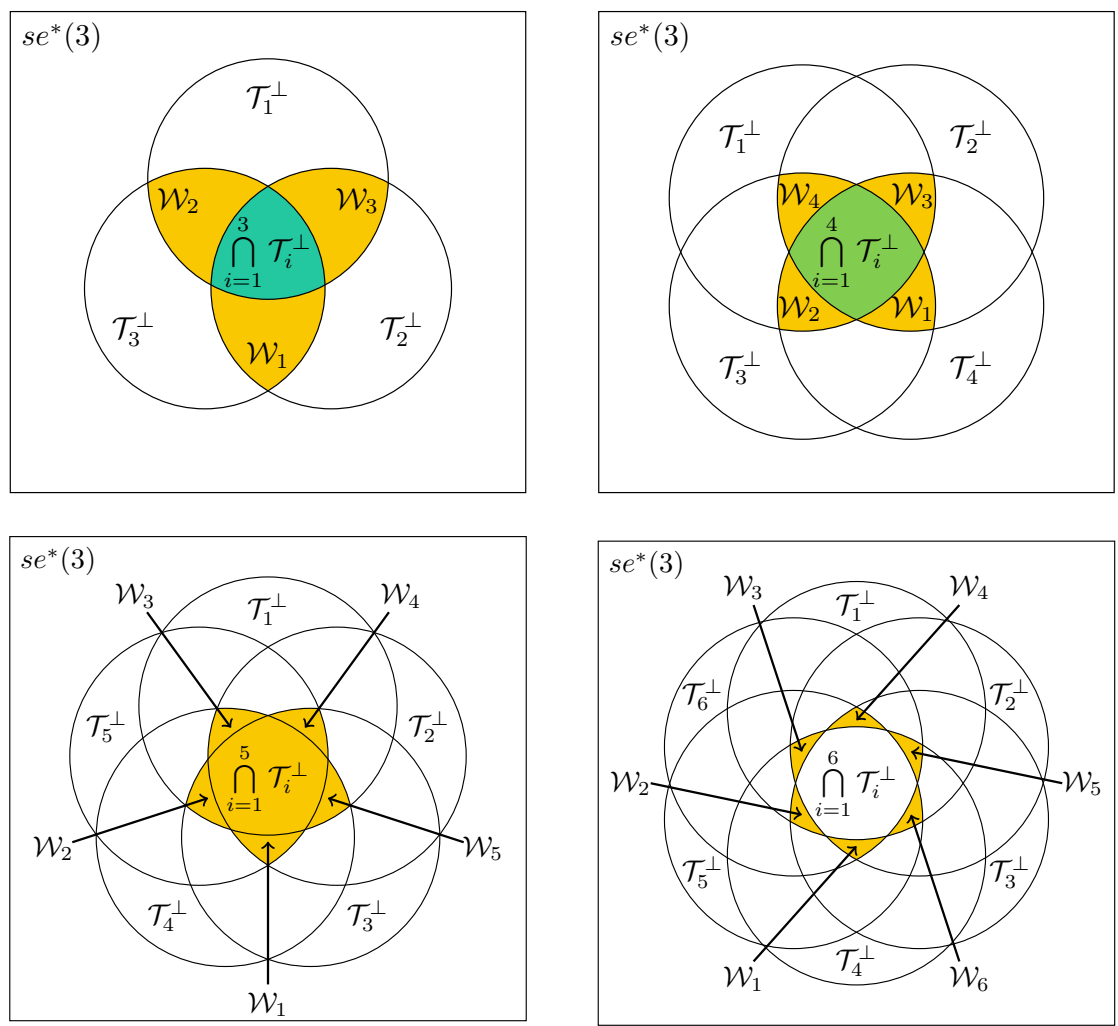

Figure 5.8: Venn diagrams illustrating the partition of $s e^{*}(3)$ into subspaces of compliant $\left(\mathcal{W}_{i}\right)$ and constraint $\left(\cap_{i=1}^{n} \mathcal{T}_{i}^{\perp}\right)$ wrenches acting on the end effector of a serial linkage with $n$ independent basic compliant joints. The highlighted areas hereby denote real subspaces with dimensions greater than zero. Since the joints are independent, the compliant spaces $\mathcal{W}_{i}$ are spanned by a unique unit wrench, and have dimension one. The space of constraint wrenches $\cap_{i=1}^{n} \mathcal{T}_{i}^{\perp}$ on the other hand, drops rank for each independent compliant joint, until the maximum number of independent joints, six, is reached, such that $\operatorname{dim} \cap_{i=1}^{n} \mathcal{T}_{i}^{\perp}=6-n$.

The transformation of the joint stiffness, on the other hand, is not intrinsic and requires the inversion of the Jacobian map. This is not possible in general, and requires the manipulator to be non-redundant and in a non-singular configuration, or the use of a pseudo-inverse of the Jacobian map, which is physically not meaningful without suitable norms [160].

It is furthermore obvious the total compliance of serial springs is obtained by summing the individual compliances, and the stiffness of parallel springs, by summing the stiffnesses. 


\subsubsection{Projection Operations}

It was shown that compliant joints partition the workspace of a robotic manipulator into complementary elastic and constrained subspaces. This implies that linear operators, so called projectors, exist, that uniquely decompose twists and wrenches into their constrained and compliant components. Given a vector space $\mathcal{V}$, with two complementary subspaces $\mathcal{A}$ and $\mathcal{B}$, such that $\mathcal{V}=\mathcal{A} \oplus \mathcal{B}$ and $\mathcal{A} \cap \mathcal{B}=\emptyset$. Then for each vector $v \in \mathcal{V}$ there are two unique vectors $a \in \mathcal{A}$ and $b \in \mathcal{B}$ such that $v=a+b$, where $a$ is the projection of $v$ onto $\mathcal{A}$ along $\mathcal{B}$, and $b$ is the projection of $v$ onto $\mathcal{B}$ along $\mathcal{A}[172]$.

Two projectors $P: \operatorname{se}(3) \rightarrow \mathcal{T}$ and $P_{\perp}: \operatorname{se}(3) \rightarrow \mathcal{W}^{\perp}$ can thus be defined, that project an end effector motion into the space $\mathcal{T} \subset \operatorname{se}(3)$ of compliant and $\mathcal{W}^{\perp} \subset$ $s e(3)$ constrained twists respectively, such that

$$
P(T)=T^{e}, \quad P_{\perp}(T)=T^{r} \quad \forall T \in \operatorname{se}(3)
$$

where $T^{e} \in \mathcal{T}$ and $T^{r} \in \mathcal{W}^{\perp}$. The transpose of these projectors can be applied to wrenches $W \in s e^{*}(3)$, such that

$$
P^{T}(W)=W^{e}, \quad P_{\perp}^{T}(W)=W^{r} \quad \forall W \in s e^{*}(3)
$$

where $W^{e} \in \mathcal{W}$ and $W^{r} \in \mathcal{T}^{\perp}$ are the elastic and constrained components of the generalized forces $W \in s e^{*}(3)$ applied to the end effector. These projectors can be computed according to [172], as soon as bases for the subspaces have been chosen.

It can furthermore be seen, that the kernel of a projector corresponds to the dual complement of its transposed [173]

$$
\operatorname{ker}(P)=\operatorname{Im}\left(P^{T}\right)^{\perp}
$$

The projection operations defined for twists and wrenches, can also be applied to higher order tensors, such as the compliance tensors. The projection can be used to map a desired compliance $\hat{C}$ onto a compliance $\bar{C}$ in the space of achievable elastic compliances of the given manipulator

$$
\bar{C}=P \hat{C} P^{T}
$$

Such projectors can then be used to formulate tasks in the compliant subspaces of the manipulator's workspace, and thus to better exploit its passive compliant properties. For a more detailed analysis they can also be composed as consecutive projection operations, defined through the workspace partitions of individual compliant joints.

\subsection{Compliance Metric}

A compliance metric is needed to compare compliance matrices, in order to quantify to which degree a given end effector compliance approximates a desired compliance 
value. Several matrix norms exist, such as induced and entry-wise norms [174], but have shortcomings. An induced norm is based on a norm of the underlying vector space, which in this case are the spaces of twists and wrenches. These spaces do not have an intrinsic norm, and any norm defined on the space is generally not physically consistent, as elements of these vectors may have different physical natures, for instance rotational and translational, leading to physically meaningless sums and units $[175,160]$. Entry-wise norms, like the Frobenius norm, treat $m \times n$ matrices, e.g., linear maps, as an $m \times n$ vector. Regular vector norms can then be applied, but the physical meaning of the metric is lost.

A geometric approach is chosen here in stead, which compares the compliance ellipsoids, as proposed for the stiffness approximation metric in [152]. A compliance tensor can be represented geometrically through a singular value decomposition $C=U \Sigma V$, where $U$ and $V$ can be viewed as rotation matrices representing the ellipsoid's orientation, and the matrix of singular values $\Sigma$ represents the "lengths" of its axes [172] where there may be an intrinsic concept of length without having defined a norm. On the other hand, two compliances, we can combine them in a coordinate free way to define a linear operator for which eigenvalues are invariant.

Consider the linear map $\bar{C}^{-1} C$ composed of the manipulator's compliance $C$ and the desired compliance tensor $\bar{C}$, an endomorphism from the space of end effector wrenches $s e^{*}(3)$ onto itself. If both compliances are identical, then $\bar{C}^{-1} C=I$ is the identity map with eigenvalues $\lambda_{i}=1$. The compliance approximation metric, defined as

$$
\mu=\sum_{i=1}^{6}\left(1-\lambda_{i}\right)^{2}, \quad \lambda_{i}=\operatorname{eig}\left(\bar{C}^{-1} C\right)
$$

thus returns a positive metric value, corresponding to the approximation error, if the compliance matrices differ, and $\bar{C}^{-1} C$ has eigenvalues not equal to one.

Because $C$ and $\bar{C}$ are tensors, the eigenvalues of $\bar{C}^{-1} C$ are invariant under coordinate system changes [176] and also not affected by user defined metrics, used for instance in the weighted Moore-Penrose pseudo-inverse [177], which have an arbitrary quality about them due to the choice of weight matrices. In order to ensure the invertibility of $\bar{C}$, the desired compliance should be already chosen as such, as we can not assume the realized compliance to be invertible.

The proposed compliance metric can be used to optimize the joint compliances, manipulator configuration, or actuation topology, with respect to the approximation error of the desired and achieved compliance ellipsoids.

\subsection{Experiments}

The SHERPA arm, a hybrid rigid-compliant serial manipulator, presented in this thesis, lends itself as case study to examine the performance of the compliance metric. The manipulator was developed to achieve a variable mechanical end effector com- 
pliance facilitating a range of dexterous interaction tasks. It has seven actuated dofs, three of which are equipped with a variable mechanical compliance: the shoulder joint $q_{2}$, and the two most distal dofs in the wrist of the manipulator, $q_{6}$ and $q_{7}$, which have a mechanically coupled compliance, as described in Chapter 3.

The task considered here is to approximate a desired Cartesian end effector stiffness as closely as possible, while the tool center point is held in a position $40 \mathrm{~cm}$ in front, and $10 \mathrm{~cm}$ above the base of the manipulator. This corresponds to the following joint positions, expressed in degrees:

$$
q_{\text {task }}=\left(\begin{array}{lllllll}
0 & 15 & 0 & 140 & 0 & 25 & 0
\end{array}\right)^{T}
$$

which resembles a range of practical manipulation tasks. While several approaches to compliance optimization are possible, including altering the pose of the manipulator, or exploiting possible null space motions in the case of a redundant system, usually tracking a certain position trajectory is prioritized. It is therefore investigated here how the values of the variable compliance joints should be tuned, even if it is not necessarily the only criterion.

\subsubsection{Compliance Control}

As an example, a desired translational compliance, acting only on translational displacements rather than complete twists,

$$
C_{d}=\left(\begin{array}{ccc}
8 & 0 & 0 \\
0 & 2 & 0 \\
0 & 0 & 2
\end{array}\right)
$$

with units in $m m / N$ has been chosen to realize a compliant behavior in $x$-direction while giving tighter guidance along the $y$ and $z$-axes, and approximated with the metric introduced in Equation (5.39). Figure 5.9 shows the value of this metric over a range of values of shoulder and wrist compliances. As can be seen, there clearly exists a value for the wrist compliance $c_{\text {wrist }}$ that minimizes the metric, and thus leads to an optimal approximation of the workspace compliance. For the shoulder compliance on the other hand, no clear minimum can be discerned, and the influence of this joint towards minimizing this particular approximation error is negligible.

In order to validate the compliance approximation metric, the compliances for the shoulder and wrist joints of the SHERPA manipulator have been set to $0.014 \mathrm{rad} / \mathrm{Nm}$ and $0.0015 \mathrm{rad} / \mathrm{Nm}$ respectively, which minimizes the metric in the case of the wrist joint, and ensures a reasonable passive deflection of the shoulder joint under gravity preload.

The compliance was measured according to the methodology presented in Section 3.7.1, where the manipulator's end effector was manually deflected. The wrenches applied to the end effector were hereby measured with an ATI F/T Mini40 6-DOF 

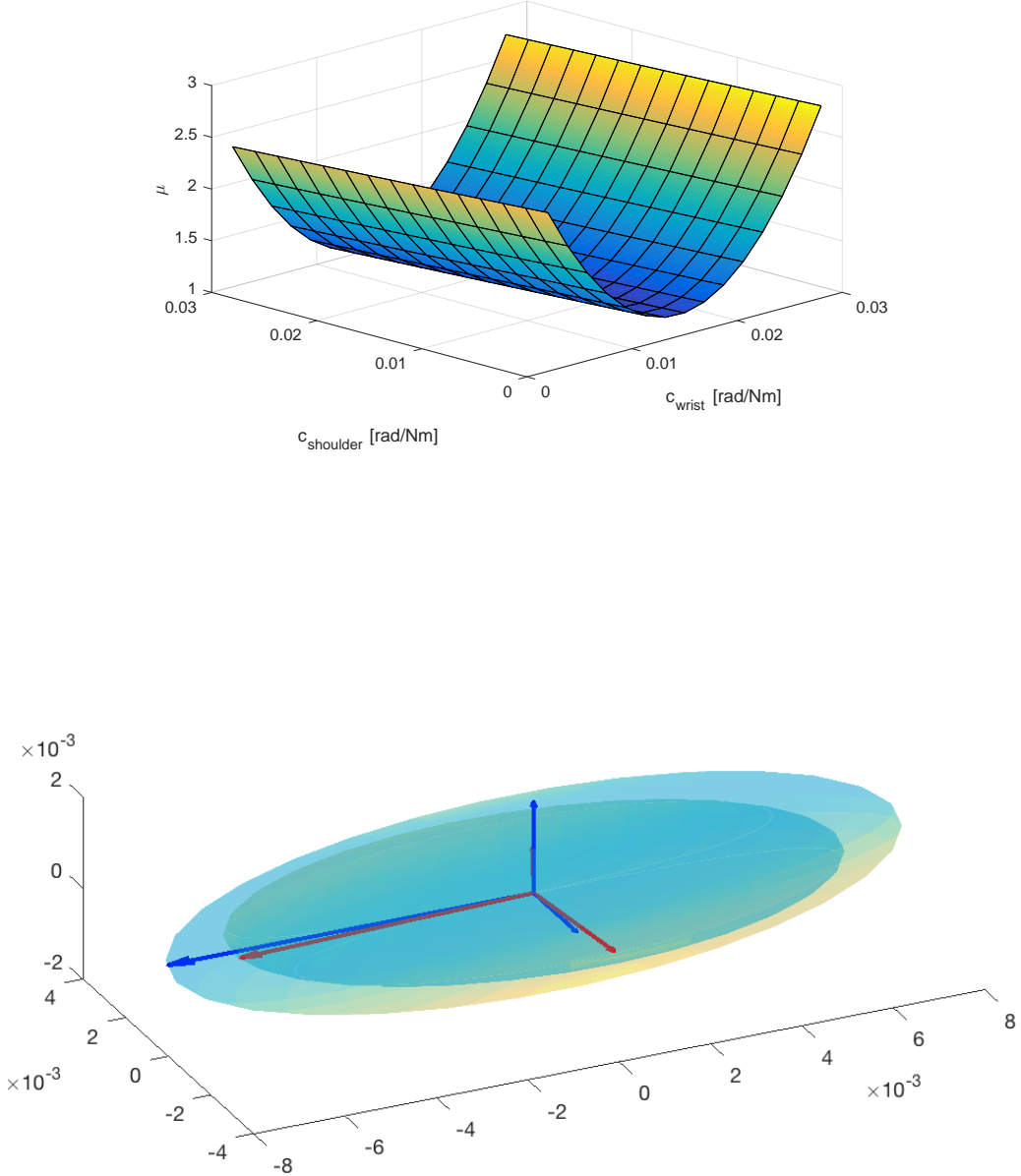

Figure 5.10: The desired and measured compliance ellipsoids and their semi-axes, as obtained through the singular value decomposition of the translational part of the compliance matrices. Even though the ellipsoids are not identical, they overlap to a large degree (quantified by the compliance approximation metric (5.39)), which may be sufficient for a given task.

force-torque sensor [138], and its position through the manipulator's integrated encoders.

Compliance ellipsoids can be visualized in an intuitive way by making use of the singular value decomposition, which was also used to derive the metric $\mu$ in the previous section. The singular values hereby represent the length of the ellipsoids' semi-axes. The ellipsoids representing the desired translational compliance $C_{d}$ and the measured compliance are shown in Figure 5.10. Even though the ellipsoids do 
not completely overlap, which was to be expected, as the Figure 5.9 shows that the approximation error does not reach zero, they match to a large degree, and the desired compliance is matched optimally with respect to the metric, which may be sufficient for a given task.

\subsubsection{Discussion}

The experiments performed with the SHERPA arm validate the metric's ability to approximate a desired end effector compliance by tuning the values of the joint compliance. While Figure 5.9 shows that one of the variable compliance joints of the arm has only negligible influence on the workspace compliance, this does not necessarily hold true in general. The end effector compliance is highly dependent on the configuration of the manipulator, such that the variable shoulder compliance may have a greater influence in shaping the system's mechanical impedance for other tasks.

Apart from providing a tool to optimize the joint compliances, the presented metric can also be used to derive design guides for the actuation topology of compliant manipulators. By quantifying the compliance approximation error it can be used to compare different manipulator designs, and their suitability for particular tasks, potentially leading to more capable and less complex systems.

\subsection{Conclusion}

This chapter presented a formulation for a compliant manipulator's configuration and workspace, as well as the kinematics of its end effector. Based on this, the kinematics and workspace partition into spaces of compliant and constrained twists and wrenches have been formulated first for a single basic compliant joint, and then extended to a hybrid serial linkage with both rigid and compliant joints. This has been done in an intuitive and coordinate free manner, using a differential geometry approach.

The formulation of the workspace partition improves the understanding of the way constrained and elastic degrees of freedom compose the spaces of compliant and constrained end effector forces and motions. The dimensional analysis of the obtained subspaces hereby highlights the precise contribution of each compliant joint. This is of interest not only from a conceptual point of view, but also has direct implications for the design and control of such mechanisms. The projection operations described in Section 5.4.4 can be used to formulate manipulation and interaction tasks in such a way that explicitly utilizes the manipulators mechanical compliance, and thus answers this thesis' research objective RO4.

Likewise, the compliance metric proposed in Section 5.5 has direct applications to the analysis, design, and control of hybrid compliant manipulators. Section 5.6.1 showed that it can be used to optimize the joint compliance for a given task, but it can also be applied to controlling other task parameters, or used to evaluate and compare different actuation topologies in the manipulator's design phase, thus addressing RO3. 
The demonstrated control of the SHERPA arm, also contributes to the engineering objective EO2 of this work, which includes the control of the arm, and its application to manipulation tasks.

Overall, this chapter presents an intuitive and innovate formulation of compliant and constrained end effector motions and forces in a robust and meaningful mathematical framework. The results of which not only find a direct application on the SHERPA arm, but also have useful applications in the design and control of semi compliant mechanisms in general. 



\section{CHAPTER 6}

\section{Autonomous BATtery EXCHANGE of UAVs With A Mobile GROUND BASE}

This chapter presents the autonomous battery exchange operation for small scale UAVs, using a mobile ground base that carries a robotic arm and a service station containing the battery exchange mechanism. The goal of this work is to demonstrate the means to increase the autonomy and persistence of robotic systems without requiring human intervention. The design and control of the system and its components are presented in detail, as well as the collaborative software framework used to plan and execute complex missions. Next to the integration of the manipulator with the SHERPA platform and control framework, as required by the engineering objective EO4, this chapter addresses the final research objective RO5 by detailing the way in which complex collaborative tasks are formulated, delegated, and executed within the SHERPA framework. Finally, the results of autonomous outdoor experiments are presented, in which the ground rover successfully localizes, retrieves, services, and deploys the landed UAV, proving its capacity to extend and enhance autonomous operations.

This chapter has been previously published as:

E. Barrett, M. Reiling, S. Mirhassani, R. Meijering, J. Jager, N. Mimmo, F. Callegati, L. Marconi, R. Carloni, and S. Stramigioli, "Autonomous Battery Exchange of UAVs with a Mobile Ground Base." in Proceedings of the IEEE International Conference on Robotics and Automation, 2018. 


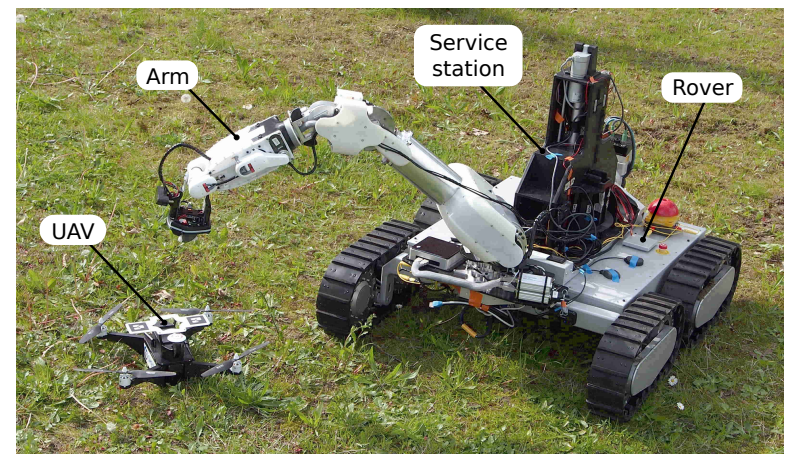

Figure 6.1: The SHERPA mobile ground base for autonomous battery exchange.

\subsection{Introduction}

Autonomous robotic platforms are increasingly utilized to execute monitoring [178], inspection [2], search and rescue [12], or disaster response missions [179]. Just as their assignments are growing more numerous and complex, the autonomous capabilities of the robotic system handling these tasks need to evolve with them, to enable new applications and to relieve their human operators of menial or dangerous work.

This chapter focuses on the autonomous battery exchange of small-scale quadrotor UAVs, which enables them to carry out persistent missions without relying on human operators to recover or recharge them. A mobile service station, mounted on a ground rover and equipped with a robotic arm to retrieve the UAVs, as shown in Figure 6.1, is chosen in the context of the SHERPA project, as outlined in Section 1.1. The state of the art and the main design choices for providing the supportive infrastructure for the small scale UAVs are introduced in the following.

1) Power Replenishment: The UAV's energy supply can be replenished either by recharging or by exchanging the depleted batteries. Several design concepts for both recharging and exchanging the batteries are in [180].

Automated battery recharging stations are the most common solution due to their lower complexity, but also show a lower vehicle utilization due to the long recharging time $[181,182,183]$. On the other hand, a battery exchange station needs a mechanism that can extract and replace the batteries from the UAV, as well as a storage mechanism to hold the spare batteries, but requires no additional time to charge the batteries before the UAV is operational again, which typically takes several times the flight time. The UAV needs to be designed such that the battery is easily exchangeable but still mounted well enough to withstand the vibrations experienced in flight and landing. Battery exchange stations, that combine rotating battery magazines with at least one linear actuator to move the battery containers from the UAV into the magazine and vice versa are presented in [184, 185, 186], while [187] uses linearly arranged 
battery bays.

As analyzed in [180], recharging stations are more economical for low coverage scenarios in terms of the provided coverage, i.e., how many UAVs are operational at a given time, versus the total system cost. Nonetheless, we chose a battery exchange system to minimize the amount of UAVs and service stations to be transported to the mission areas.

2) Service Provision: To service the UAVs in a dynamic mission with shifting areas of interest and, thus, to extend their operational radius, we chose a mobile ground base, as the strategical placement of stationary service stations across the operational environment $[181,188]$ is not a practical solution for search and rescue or emergency response operations. In $[182,183]$ recharging stations have been mounted on commercial mobile ground vehicles, but these platforms were intended for laboratory environments. Instead, a tracked vehicle with robust outdoor navigation capabilities was developed for the SHERPA project (BlueBotics SA, Switzerland).

3) UAV Retrieval: The service stations presented in the literature require the UAV to precisely and safely land on a landing pad on the station. Most of the time, however, the landing precision of the UAV is not sufficient to engage the replenishment mechanism, also due to wind. This is especially the case for battery exchange mechanisms, where a close mechanical fit is required. To overcome the alignment issue some of the proposed stations have passive guidance systems that function like funnels [180, 185], while others are equipped with active alignment systems, like small arms or wire mechanisms $[186,188]$ that position and secure the UAVs during replenishment. However, many systems also still rely on external position sensing in order to land the UAV on the platform.

While these systems alleviate the problem of retrieving the UAVs, the landing operation is still delicate. The SHERPA mission requires that the robotic platform can reliably retrieve the UAVs without human involvement. Hence, we chose to mount a robotic arm on the ground rover, that is capable of robustly retrieving, docking, and deploying the UAVs. In order to increase robustness during the manipulation and docking of the UAVs, the arm is designed with a variable mechanical compliance, as presented in Chapter 3.

4) Software Framework: Complex missions involving a heterogeneous robotic team also necessitate a suited control and communications structure $[189,190]$. A framework for the automatic specification, generation, and execution of high-level collaborative mission plans has been presented in [191] as part of the SHERPA project, which is a more robust and flexible solution than systems based on a central planning and scheduling algorithm [181, 192].

The remainder of this chapter is organized as follows. Section 6.2 outlines the software framework used by the agents of the system to plan and execute tasks, while Section 6.3 presents the design of these agents in detail. Experimental results on the autonomous battery exchange are presented in Section 6.4, and concluding remarks are drawn in Section 6.5. 


\subsection{Collaborative Mission Framework}

Complex collaborative tasks that would be difficult or impossible to perform for individual agents require the cooperation and coordination of the participating agents. This section presents the distributed communication and control architecture, that enables a larger heterogeneous human-robot team to effectively work together in a robust and versatile manner, even under adverse operating conditions. The delegation framework and semantic structures developed for the SHERPA mission, as described in [191], are designed to enable a team of robotic agents to cooperate in planning and executing complex and hierarchical tasks in a dynamic mission environment. An overview of the delegation process and the agents and components involved in the battery exchange are shown in Figure 6.2.

A generic task or mission goal, such as the exchange of the UAV's battery, is expressed in the form of a goal request TST (Task Specific Tree), i.e. a hierarchically structured description of the task. The internal nodes of a TST represent control statements for the task's execution, while leaf nodes represent domain specific functionalities. The transformation of a high-level request into a goal request TST takes place during the mission by dynamically instantiating generic TST templates.

The involved agents interact through their delegation modules, which contain a TST factory that can create TST nodes and link them to ancestors and descendants across agents, as well as a TST Executor Factory that provides platform specific functionalities for the execution of a task. The delegation module and interaction protocols are based on ROS.

In the first phase of the delegation, a goal request TST is negotiated. When an agent requests a goal, the goal request TST is sent to its delegation module, where the distributed delegation process allocates each node of the tree to a suitable agent by means of an auction. The most suited bidder for a task is determined by solving the constraint problem belonging to a cost function, taking into account the mission requirements and platform capabilities.

The result of a successful delegation is an expanded collaborative plan TST, where all nodes have been allocated to the participating agents. This TST can then be executed in the second phase of the delegation. The autonomous execution of the battery exchange operation is described in Section 6.4.1 as an example of such an expanded collaborative plan TST.

\subsection{The SHERPA System}

In this section, the hardware and the control of the agents, i.e., the robots involved in the battery exchange, are described in detail. More specifically, the UAV (Unmanned Aerial Vehicle) (wasp), the SHERPA box (SBox) service station, the mobile ground rover, and the robotic arm are involved in the operation. In some cases the rover 


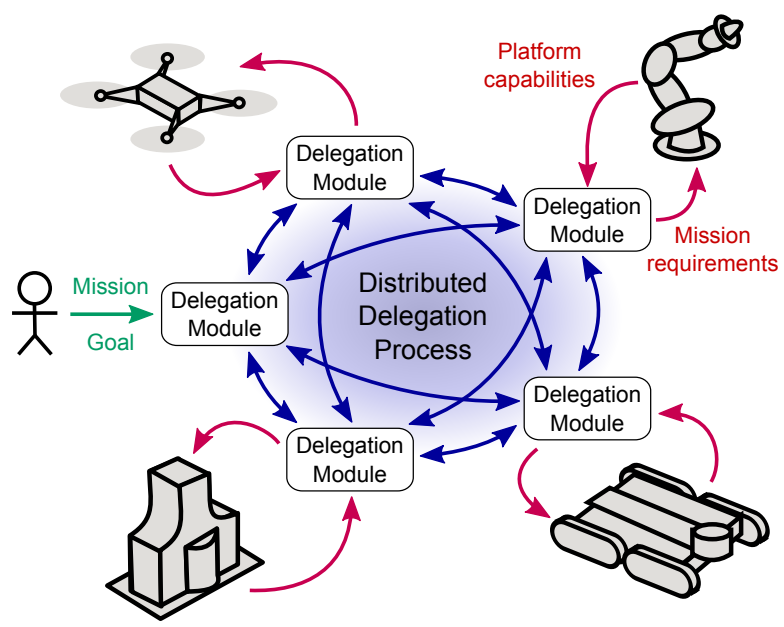

Figure 6.2: Overview of the distributed delegation process and of the agents for the autonomous battery exchange during the SHERPA mission. Each agent has a delegation module that mediates between mission requirements and platform capabilities. The human operator can request or approve mission goals, but the system is also able to request, plan and execute missions completely autonomously without human supervision in a distributed delegation process, in which tasks are assigned to the most suited agents.

and arm are seen as a single agent for the higher-level planning, for instance when performing coordinated actions. They are then referred to as ground-rover-arm (GRA).

\subsubsection{Small-Scale UAVs - The SHERPA Wasps}

The role of the wasps, shown in Figure 6.3, is to gather data of the environment through cameras or other specialized sensors. Their high maneuverability and ability to hover over or follow targets makes them ideally suited for this task, even in otherwise inaccessible terrain. Due to their small size, low payload, and a flight time of approximately 20 minutes they are dependent on support from a ground station.

This reliance on a service station, and the requirement of a robust and efficient battery exchange mechanism, has resulted in a highly integrated interdependent design of the wasps and the SBox (ASLATECH, Italy). Rather than using a modular external battery carriage receptacle $[184,187]$, the battery bay is integrated into the structural frame of the wasps. This design leads to a stronger and lighter construction, while at the same time shielding the batteries and connectors from the environment, and protecting them from exposure to dirt, water, or mechanical influences. The battery carriage is made of a carbon fiber container that can slide in and out of the battery bays in the wasps and SBox. The UAV's main body furthermore contains the flight control module, a computer for high-level control, and a signal interface board, while the motor controllers are contained in the four arms holding the motors. 


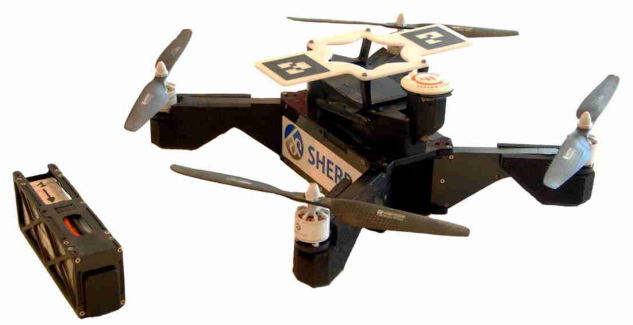

Figure 6.3: The small scale UAV - the SHERPA wasp - and one of the exchangeable batteries

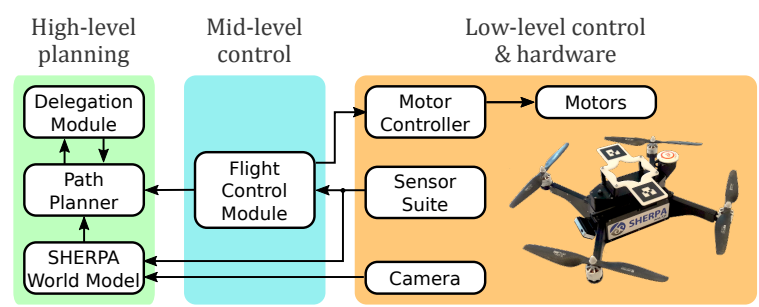

Figure 6.4: System architecture of the SHERPA wasps.

As shown in Figure 6.4, the control of the wasps is organized in two main parts; a high-level planning module, and a mid-level flight control module. The high-level planner interacts with the delegation framework and includes a navigation path planner, which translates requested tasks into waypoints of suitable trajectories. The midlevel flight control is running on a real time operating system and interfaces the sensors and actuators. It runs stabilization algorithms that reject external disturbances such as wind, tracks the waypoints supplied by the path planner, arms and disarms the motors, and is capable of autonomous take-off and landing.

\subsubsection{Service Station - The SHERPA Box}

The SHERPA box is designed to function as the service station for docking and replenishing the wasps, as well as the computational and communications hub for the mission. The battery exchange mechanism itself consists of a linear actuator that slides the battery carriages from the UAV into a revolving battery magazine at the base of the SBox and vice versa. A sectional view of the SBox and the wasp is shown in Figure 6.5 , displaying the components of the mechanism. Since the wasps do not have to land on the SBox, the battery exchange mechanism can be arranged vertically, which allows to service two wasps simultaneously.

For docking the wasp on the SBox, the wasp is initially placed on two small guide rails, before it is moved into its final docking position, guided by the rails and a short 


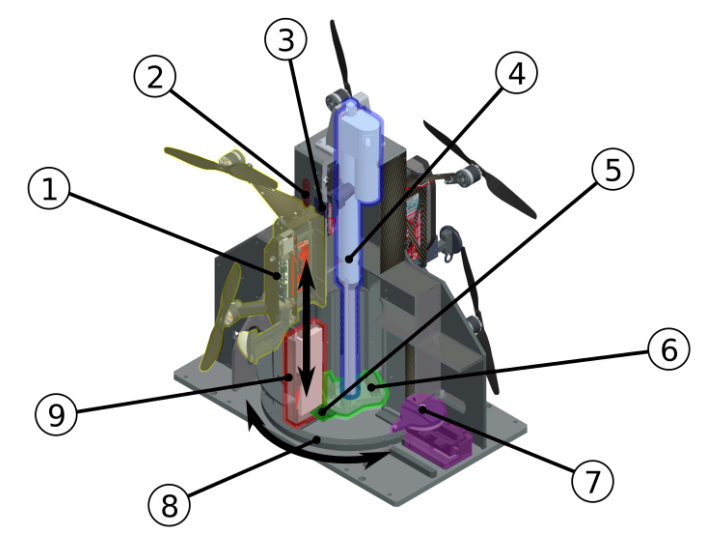

Figure 6.5: A partial section view of the SBox and docked wasps showing the battery exchange mechanism. The wasp (1) is locked to the SBox with the clamps (2) when the switch (3) is engaged. To remove the battery (9) from the wasp a 'tongue' (5) engages the battery, which is pulled downwards into the battery magazine (8) when the linear actuator (4) moves the toolhead (6). To exchange the battery, the magazine is rotated by the actuator (7) which drives a Geneva mechanism. The battery exchange mechanism then places a charged battery into the wasp.

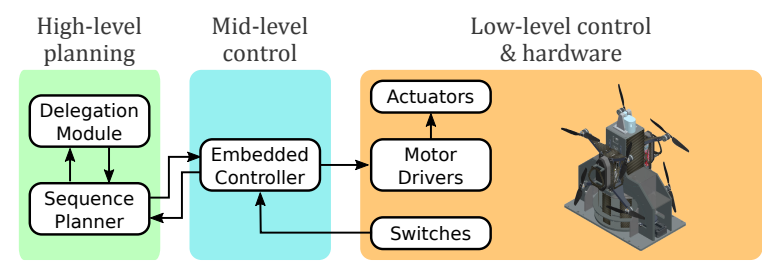

Figure 6.6: System architecture of the UAV service station and communications hub - the SHERPA box.

funnel. Once the wasp has been placed correctly, a switch registers that the wasp is docked, and two small clamps lock it securely in place. A tongue then extends from the toolhead at the end of the linear actuator, and engages a notch in the battery carriage, in order to move it downwards into a free slot of the battery magazine. The battery slots of the magazine are aligned with the battery compartment of the wasp by means of a Geneva mechanism, which rotates the magazine in discrete steps ensuring proper alignment, after the tongue has disengaged the empty battery. When the charged battery has been aligned, it is engaged by the tongue, and placed into the battery bay of the wasp, where it securely engages the electrical connectors and locks into place. The replenished wasp is then ready to be released by the SBox and to continue its mission.

As shown in Figure 6.6, the SBox has a hierarchical control structure. A high-level sequence planner and delegation module, which run on an Intel NUC, coordinate with the delegation framework. They translate requests for the Arduino-based embedded 


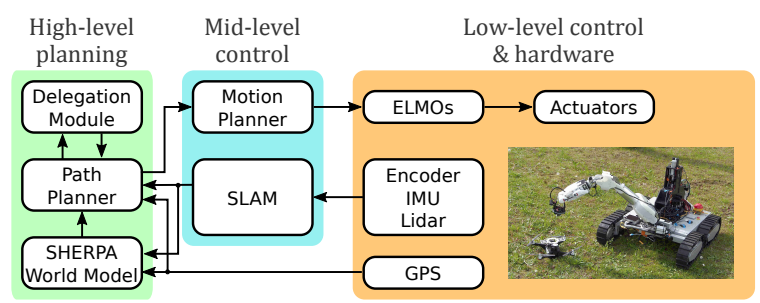

Figure 6.7: System architecture of the mobile base station (ground rover).

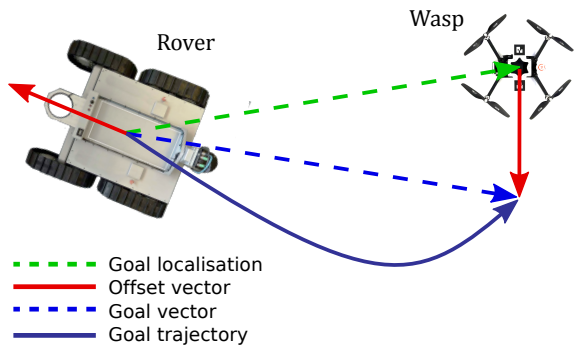

Figure 6.8: Trajectory planning for the rover's approach phase through goal vector summation and planning algorithm. The goal localization is performed when the rover detects the wasp and adds it to the map. Adding the offset vector to that position results in the desired goal vector of the rover with the correct distance and orientation to the wasp. Finally a collision free trajectory is generated.

controller that interfaces with the sensors and actuators of the SBox.

\subsubsection{Mobile Base - The Ground Rover}

A specially developed (BlueBotics, Switzerland) ground rover serves as mobile base for the SBox and the robotic arm. It is characterized by its high degree of autonomy, endurance, and payload capacity. The rover is driven by four actuated tracks, that are mounted on a passively configurable chassis. These tracks allows the rover to traverse rough, mountainous terrain at a maximum speed of $0.8 \mathrm{~m} / \mathrm{s}$. Its range of sensors include a tilting laser scanner (lidar), IMU and GPS systems, as well as encoders for the tracks. The system is designed to run autonomously for three to six hours, depending on the usage of actuators. The battery can be changed without shutting down the hardware. The rover is furthermore equipped with an Intel NUC computer, Wi-Fi interface and internal power electronics.

Figure 6.7 shows the system architecture of the rover, which is divided into highlevel planning, mid-level control, and low-level control and hardware. The high-level control interacts with the delegation framework, and formulates requested tasks as navigation problems that are solved by the path planner. A mid-level motion planner resolves the planned path and coordinates the individual motor drivers. On the same 


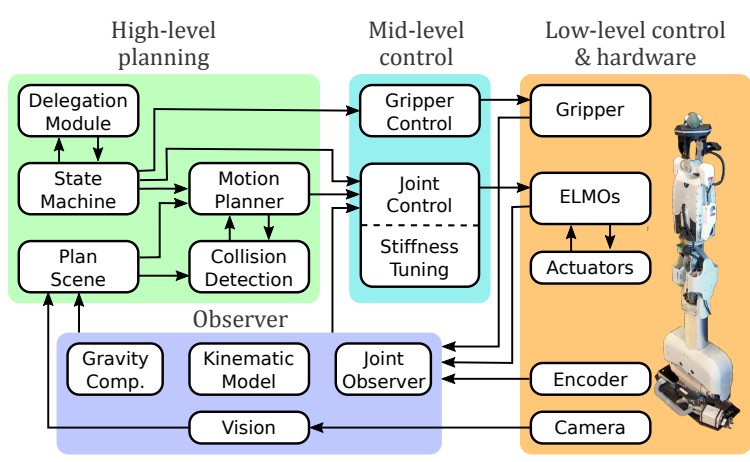

Figure 6.9: An overview of the system architecture of the arm. The manipulator's software structure is presented in more detail in Figure 3.13.

level, a SLAM module combines data from the lidar, encoders, and IMU and provides a map of the environment with the rover's position to the high level path planner. The actual execution of the trajectory, and control of the actuators, is performed by ELMO Whistle digital servo drives (Elmo Motion Control Ltd., Israel).

During the battery exchange operation, the rover needs to plan and execute a trajectory that places the wasp within the workspace of the arm. It does so by using the map generated by the SLAM module, and the position of the wasp, provided by the arm and its end-effector camera. The GPS is not used for the precise localization of the wasp due to its limited accuracy. Using the relative body-inertial frame information provided by the SLAM algorithm, the wasp is localized in the map and its corresponding position is added to the map as an obstacle to ensure the safety of the systems. Figure 6.8 illustrates the summation of the position vectors that gives the rover's desired goal position as a function of the initial positions and orientations of the rover and wasp. The collision free trajectory is generated with an $\mathrm{A}^{*}$ algorithm implemented using a 2D navigation stack in ROS, and is subsequently executed by the platform.

\subsubsection{UAV Retrieval - The Robotic Arm}

The ground rover is equipped with a robotic arm [83], in order to robustly deploy and recover the wasps, and thus to facilitate the autonomous servicing and battery exchange operation. Figure 6.9 shows the system architecture of the arm.

The arm has 7 DOF and a reach of one meter, and is designed for a payload of $2 \mathrm{~kg}$. To enable compliant and safe interaction with the wasps and SBox during grasping and docking, the arm is equipped with two variable stiffness mechanisms in the shoulder and wrist joints. The arm's end-effector is a custom made gripper, that interlocks with an interface mounted on the wasp. As the arm is able to retrieve wasps without human assistance or requiring the wasps to perform precise and sensitive landing operations, 
it greatly extends the system's robustness and autonomy.

The high-level computational tasks are executed on an Intel NUC computer, while the low-level motor control is performed by ELMO Whistle digital servo drives.

The arm's high-level planner includes a delegation module that interacts with the other agents, and a hierarchical finite-state machine (HFSM) based on the ROS decision making package, where the states of the HFSM are triggered by executors and represent the leaf nodes of the TST, i.e. the specific implementation of tasks for the arm. When a task requires the arm to move to a certain Cartesian goal, a suitable joint space trajectory is generated using the rapidly-exploring random tree (RRT ${ }^{*}$ ) algorithm implemented in MoveIt!. The mid-level joint controller then translates these trajectories to motor set-points and acts as supervisory controller.

The arm's observer combines data from the different sensors, including a Logitech c920 webcam mounted on the gripper, to obtain the state estimates. The vision-based localization of the wasps is also carried out by the arm observer. Markers placed on the wasp interface are detected using the ROS AR TRACK ALVAR wrapper. After its detection, a virtual wasp is placed in the plan scene of the arm, and its pose is published to other agents, such as the rover.

\subsection{Experiments}

This section presents the autonomous battery exchange procedure and experimental results. The experiments were performed outdoors. The individual components of the tasks were carried out by the system's agents and coordinated by the delegation framework.

\subsubsection{Battery Exchange Operation}

The execution of a collaborative plan TST, i.e. the executor, is the second phase of the delegation process presented in Section 6.2. The battery exchange operation is a relatively simple expanded collaborative plan TST, which could also be generated without the auctioning process, if the role of every agent is clear from the start. Here it thus serves as an example illustrating the structure of such a TST, while the full potential of the planning and delegation framework used here comes into play in more complex multi-agent operations. When the battery replacement executor, shown in Figure 6.10, is requested, it is delegated to the GRA agent and expands into several nodes that are delegated to the arm, wasp, rover, and SBox agents.

The change batt executor is typically triggered by either the human operator, or fully autonomously by the UAV, which will request the battery exchange after landing when its power is running low. After accepting the delegation, the GRA expands the executor and commands the arm to localize the UAV through the find_wasp executor. This executor triggers the arm to search for the UAV with its end-effector camera through coordinated motions of the arm and the rover, after which the location and 


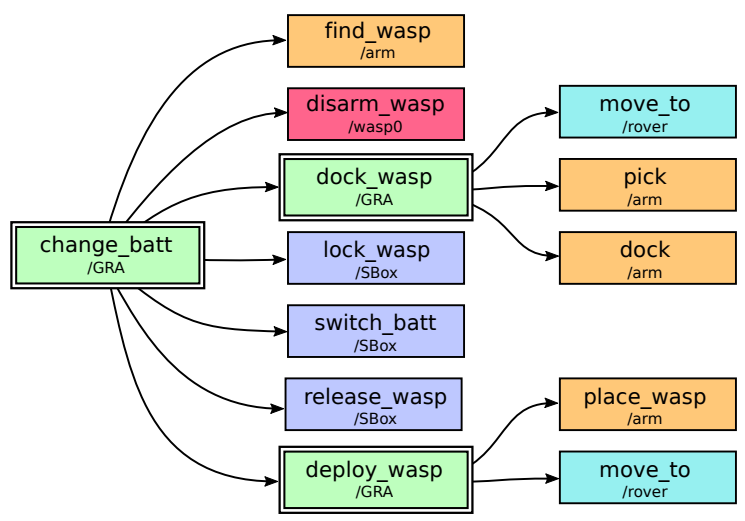

Figure 6.10: The battery change executor (expanded collaborative plan TST) is delegated to the GRA agent. It expands into other executors, which are delegated to the arm, wasp, rover, and SBox agents. Internal nodes of the executor represent control statements, leaf nodes represent domain specific tasks. The different colors denote which agent the task is delegated to.

pose of the UAV is published to the other agents. Next, the UAV is commanded to disarm its motors, and the GRA to dock the UAV. The dock_wasp executor then in turn expands into the move_to, pick, and dock executors, which command the rover to approach the UAV, and the arm to grasp and move the UAV into the docking positing on the SHERPA box. The following lock_wasp, switch_batt, and release_wasp executors are delegated to the SBox, and lock the UAV in the docking position, replace the depleted battery for a charged one, and release the UAV respectively. Finally the UAV is deployed again by placing it on the ground, and moving the GRA away from it, such that the UAV and GRA can continue their respective missions.

\subsubsection{Experimental Results}

When the GRA is delegated to exchange the landed UAV's batteries, the arm determines its precise position relative to the rover. Figure 6.11 shows the detection of the UAV's markers as described in Section 6.3.4. The still image of the camera feed is overlaid with the detected location of the marker, and the virtual collision object of the plan scene.

The virtual UAV is included in the plan scene of the arm, as shown in Figure 6.12 , and published. The rover then plans a collision-free trajectory, as outlined in Section 6.3.3, and approaches the UAV, such that the UAV is located within the arm's workspace and can easily be grasped. Figure 6.13 shows the rover and UAV locations in the map generated by the rover, and the rover's trajectory.

Figure 6.14 shows the arm docking and deploying the UAV as a sequence of still images. After approaching the UAV, the arm picks up, grasps, and docks it on the SHERPA box, where the UAV's battery is exchanged according to Section 6.3.2. The 
arm then places the UAV back on the ground and the rover moves away from the UAV so that it can continue its mission with a replenished battery. The tunable passive compliance of the arm has proven to be instrumental in the successful execution of the intricate interaction tasks. During grasping and docking of the UAV, the arm's compliance in the shoulder and wrist joints is controlled to make the operation more robust and reliable.

Figure 6.15 gives an overview of the execution of the battery exchange by presenting key variables of the rover, arm, and SBox over the course of the operation.

\subsection{Conclusions}

This chapter presented the autonomous replacement of the battery of a small-scale $\mathrm{UAV}$, in the scope of a search and rescue mission. The replacement is carried out by a heterogeneous team of robotic agents that are capable of planning and executing complex missions and tasks. This is facilitated by a software framework that is capable of coordinating a large group of diverse agents.

The successful battery exchange operation demonstrates these capabilities, thereby fulfilling the engineering objective EO4, and at the same time highlights how the endurance of small-scale UAVs can effectively be extended. By replenishing its power supply, the UAV's flight time is greatly extended, as well as its operational radius, which is further increased by the service station mobility. The autonomy of the system is enhanced by the addition of a robotic arm to retrieve the UAVs, minimizing the need for human intervention during the mission. The main contribution of this chapter is the combination of a mobile service station and robotic arm to retrieve the UAVs. The task specification and delegation framework used to execute the mission answers the objective $\mathbf{R O 5}$ to coordinate and successfully complete complex collaborative tasks. 


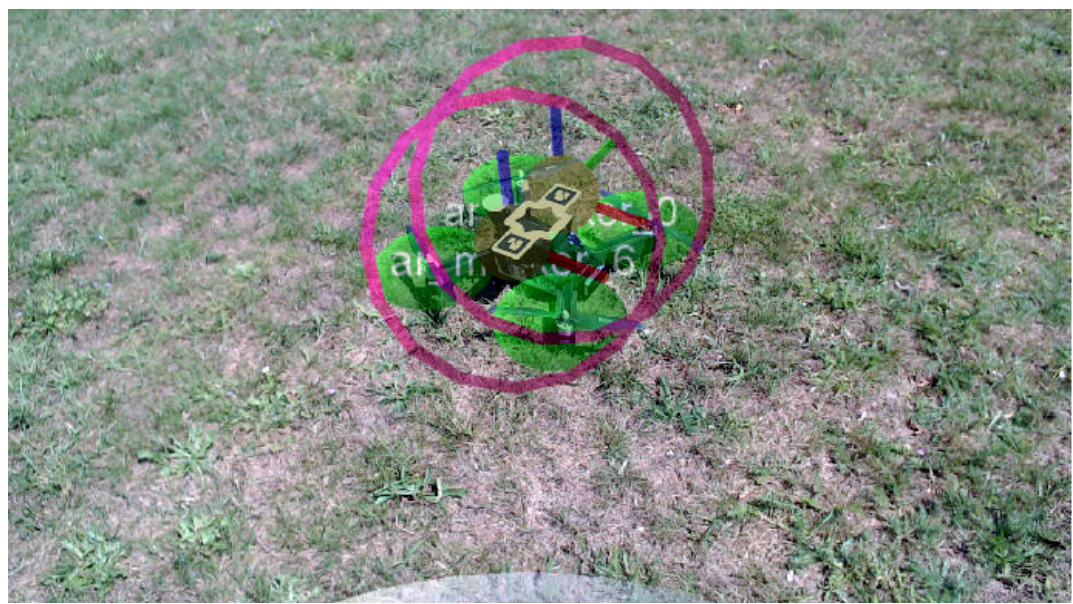

Figure 6.11: The UAV is detected from the video feed of the arm's camera by means of the markers placed on the interface. The position of the markers is denoted by the two circles, while the orientation is shown through the superimposed coordinate frames. The virtual model of the UAV (shown in green) is also overlaid onto the live video stream.

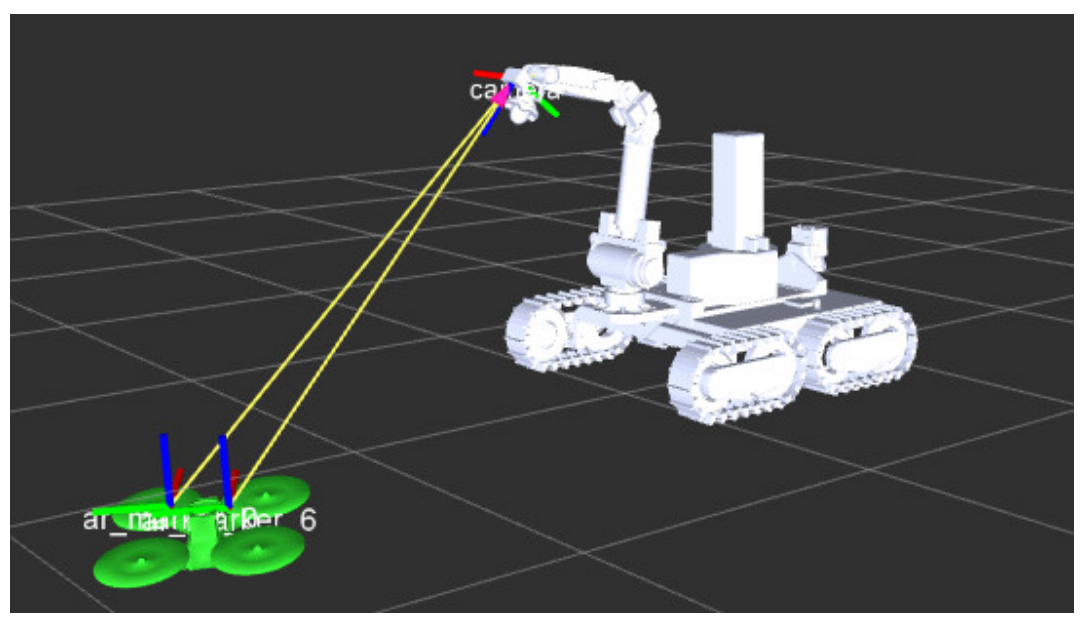

Figure 6.12: The virtual collision object of the UAV is placed in the plan scene of the arm once it is detected. The UAV's position and orientation are also published to the other agents. 


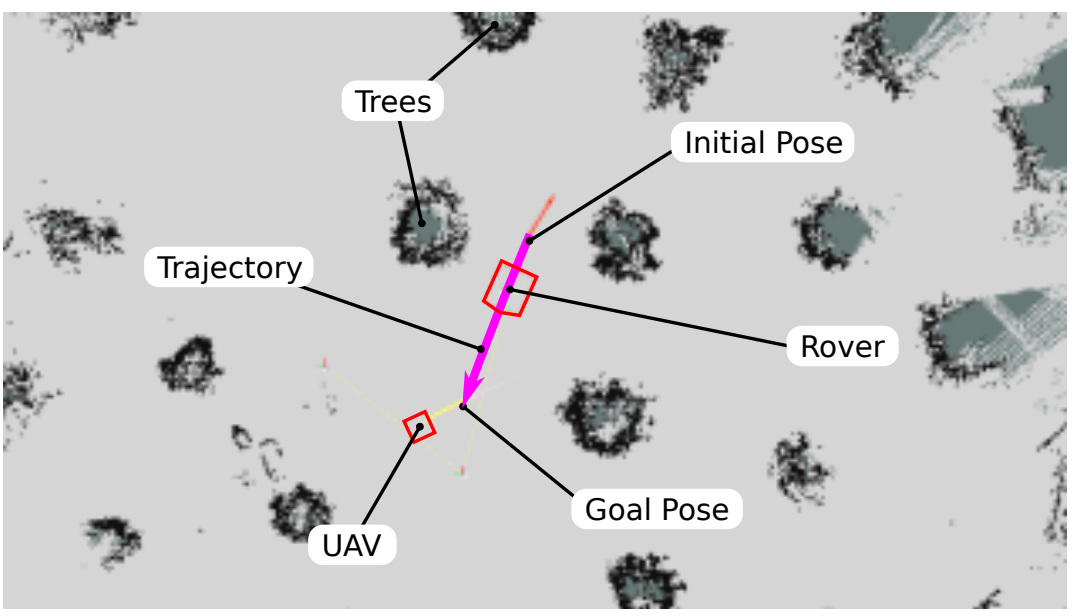

Figure 6.13: The map generated by the rover during the experiments. Free cells are shown in light gray, occupied cells in black, and unknown cells in dark gray. The small trees in the rover's environment are clearly visible as obstacles. The map shows the initial pose of the rover and its goal pose, at an offset from the UAV position, as well as the generated trajectory connecting the two.

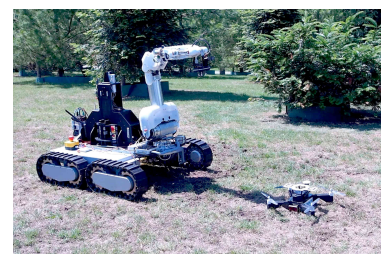

(a) Scan and approach

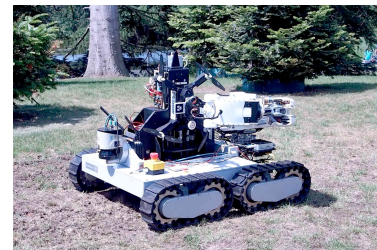

(d) Battery exchange

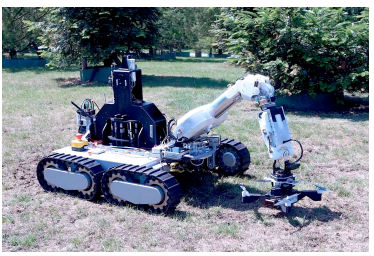

(b) Grasp

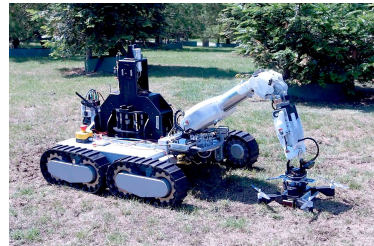

(e) Deploy

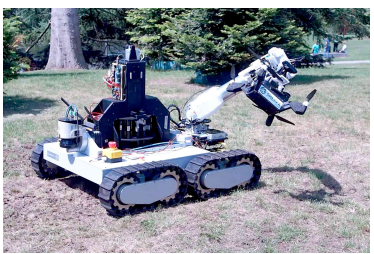

(c) Docking

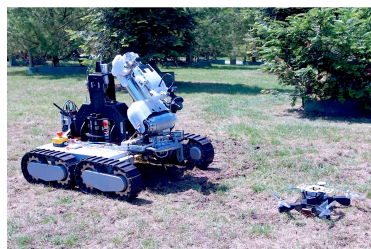

(f) Retreat

Figure 6.14: After the ground rover approaches the landed wasp (Figure 6.14a) the arm picks it up (Figure $6.14 \mathrm{~b}$ ) and docks it on the SHERPA box (Figure 6.14c) where its battery is exchanged (Figure 6.14d). The arm then places it back on the ground (Figure 6.14e) and retreats away from the wasp (Figure 6.14f). 


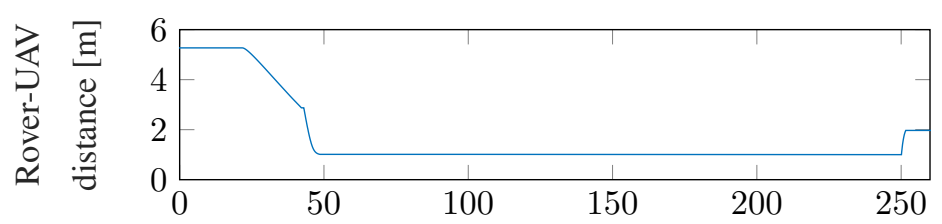

(a) Distance between rover and wasp.

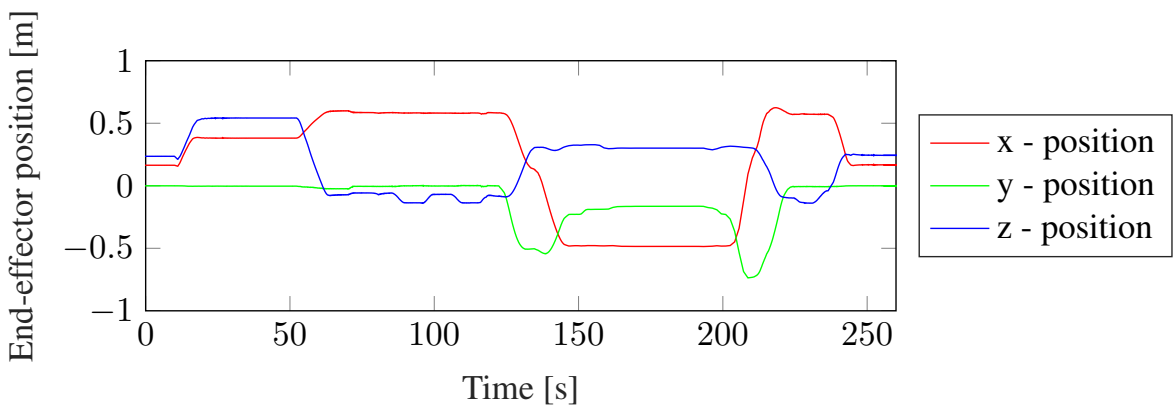

(b) End-effector position of the arm.
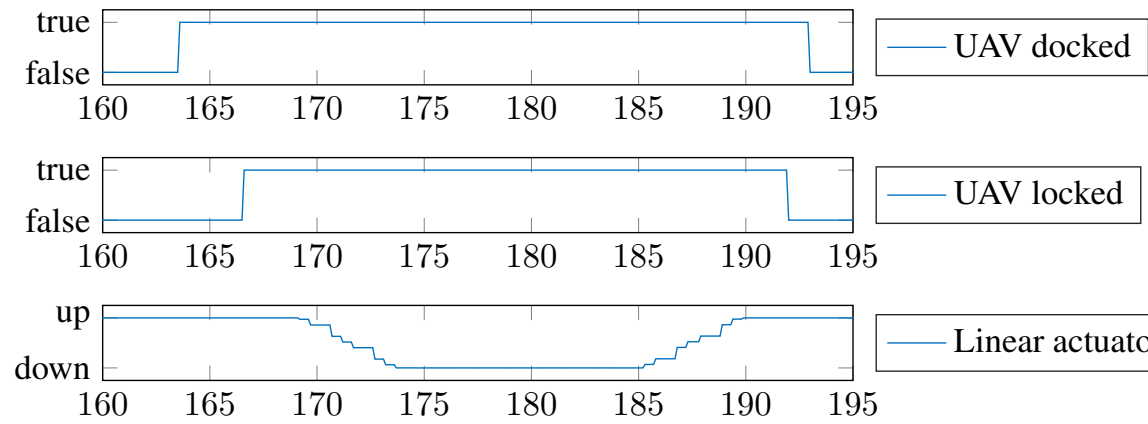

Linear actuator

$\begin{array}{llllllll}160 & 165 & 170 & 175 & 180 & 185 & 190 & 195\end{array}$

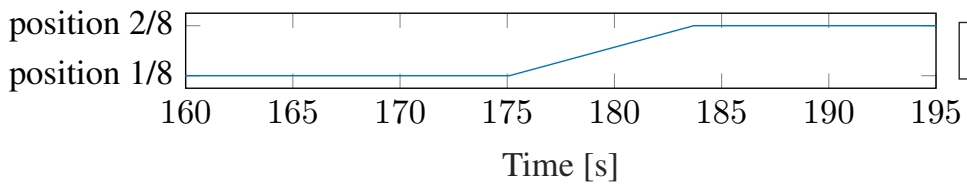

Battery container

(c) Status of the SBox.

Figure 6.15: Overview of the battery exchange operation through the status of the involved agents. Figure 6.15a shows the distance between the rover and wasp, as the rover approaches it during the execution of the dock_wasp-executor, and moves away from the wasp along the trajectory generated by the rover's path planner during the deploy_wasp-executor. Figure $6.15 \mathrm{~b}$ shows the end-effector position of the robotic arm. The arm moves first into a scanning pose during find_wasp and the start of dock_wasp, before it grasps and docks the wasp, and places it back on the ground during the deploy_wasp executor. Figure 6.15c shows the status of the SBox. The SBox registers the wasp as docked when the arm successfully places the wasp on the SBox. The wasp is then locked by the docking clamps while the linear actuator moves the empty battery into the battery container, which then moves a charged battery into position that is inserted into the wasp by the linear actuator. 



\section{CHAPTER 7}

\section{CONCLUSIONS}

Compliant and collaborative manipulators are currently making great strides towards becoming safe, reliable, and adaptable tools, opening up many new applications in which such robots can have a positive impact. However, further research and technical development is still needed on all levels of these systems, ranging from single actuators and their components, over the manipulator level, to the integration and application of these robots to complex and collaborative tasks and environments. The key objectives of this work are twofold, and can be divided into scientific research questions addressing each of these levels, on the one hand:

Actuator - level: How can the energy storage capacity of series elastic and variable stiffness actuators be maximized, and the utilization of that energy storage optimized? (RO1 and RO2)

Manipulator - level: What kind of actuation topology is needed to achieve a desired workspace compliance, while keeping the mechanical complexity as low as possible, and how can the resulting mechanical compliance best be exploited? (RO3 and RO4)

Mission - level: How can complex, collaborative tasks involving several robotic or human agents be formulated, delegated, and executed? (RO5)

and, on the other hand, more practically oriented engineering objectives:

Engineering: Conduct the design, construction, control, integration, and application of the arm and its components in the SHERPA project. (EO1 - EO4) 


\subsection{Findings and Conclusions}

This section presents an overview of the findings and conclusions of this dissertation in light of the research questions formulated in Section 1.3. These contributions are associated with the SHERPA mission, compliant actuation, and compliant manipulators, while suggestions for future work are given in Section 7.2.

\subsubsection{Compliant Actuators - VSA and Spring Design}

Interaction with a dynamic and uncertain environment should be approached through the energy, the lingua franca of physics, that a system expends and absorbs. Mechanically compliant actuators expressly include an elastic storage element to capture and reuse absorbed energy. Because robotic actuators also need to be light and compact, the inclusion of large springs and complex mechanism seems at odds with these requirements, and often the energy storage capacity remains a bottle neck for the performance of the system.

Chapter 2 addresses the research objectives RO1 and RO2 related to the energy storage capacity on the level of single actuators, by investigating how energy is routed and stored inside VSAs, and how the storage capacity can be maximized for a given system.

While SEAs can directly link their internal spring to the load, VSAs include a mechanism which can adjust the output stiffness by modulating the internal power flows. A port-based model was presented and used to investigate these power flows, and how different types of VSAs utilize their internal energy storage depending on their output stiffness setting. It was found that VSAs based on a variable transmission between their internal springs and the load, retain their storage capacity over a wide range of output stiffnesses and require no change of their spring state when adjusting their stiffness. This is in contrast with designs that are for instance based on an antagonistic setup of non-linear springs, which rely on pretensioning their internal springs in order to alter their output stiffness, and thus loose the capacity to store energy supplied by the environment, even if the choice of operating principle and spring type still depends on the specific implementation and application.

A design that implements a variable transmission through a lever arm with adjustable pivot point was chosen for further use in the SHERPA arm, and specific design requirements, that account for instance for the large internal forces occurring in such a mechanism, were derived. Chapter 2, however, also presents general design guidelines that can be used to maximize the mechanical energy storage capacity of compliant storage elements, relating to the spring's geometry and material. In designing the spring, an even stress distribution through the material should be targeted in order to maximize the degree of volume utilization. This will avoid failure due to excessive stress in isolated points, and more volume of the springs material contributes to storing mechanical strain energy. The proper choice of material is also 
essential. The material should be selected according to material indices, like the metric defined in this work, which maximizes the specific energy capacity given certain geometric constraints. Finally the overall spring volume should be maximized through proper use of the available mounting space in the actuator. The application of these guidelines lead to an $\Omega$-shaped polymer leaf spring, which demonstrated that it indeed increased the actuator's energy capacity through finite elements analysis and experiments. Even though the presented design methodology achieved a high energy storage density, overall, the addition of VSAs lead to larger and heavier wrist and shoulder joints.

\subsubsection{Compliant Manipulators - Design and Control}

The design and control of the compliant manipulator presented in this thesis, representing a hybrid rigid-compliant system, has mainly been focused on the passive mechanical properties of the system. In order to answer research questions RO3 and RO4 the manipulator's actuation topology and resulting end effector compliance have been studied, along with its application to manipulation tasks.

Chapter 3 investigated how the actuation topology of the manipulator should be composed in order to facilitate a range of end effector compliances that enable compliant and dexterous manipulation tasks in the context of the SHERPA mission. More specifically to grasp and dock a UAV in a safe and robust manner. To this end a range of compliances for different key positions was defined through their compliance ellipsoids, representing different stages of the task. To keep the system complexity low, the number of VSAs was to be kept to the minimum number needed to reach the desired variable end effector compliance. It was found that this can already be achieved with only three VSAs, one in the shoulder, and two coupled joints in the wrist, not only reducing cost and engineering effort, but also the size and weight of the manipulator, all important design criteria. The same chapter answered RO4 empirically through an iterative experimental approach. By tuning the joint compliance for specific stages of the task the procedure of grasping the UAV and docking it on the service station was made more robust. This process highlighted the usefulness of a properly tuned mechanical compliance, which enabled complex and difficult manipulation tasks with an extremely simple controller, but was not carried out with a 'hands-on' approach, and did not yet follow a formal and general analysis.

Chapter 5, by contrast, sets out to address these research objectives in a comprehensive and formal way, setting up a framework describing hybrid serial linkages with both compliant and rigid joints in a coordinate invariant manner through a differential geometry approach. The partitioning of the workspace into compliant and constrained subspaces of twists and wrenches applied to the end effector, as well as each joint's contribution to the total mechanism compliance are made clear. The proposed compliance metric can be used to evaluate and optimize the actuation topologies of different manipulator designs, providing a quantitative tool for answering research question RO3. The same chapter also proposes projection operations, through which 
interaction tasks can be formulated in such a way that they take place in the compliant subspaces of the workspace, exploiting the passive compliance, and thus provides the tools to answer RO4. Even though they have not been fully implemented, the results presented in Chapter 5 find a direct application in the design and control of the SHERPA arm, and also provide the means to analyze, design, and control mechanically compliant manipulators in general.

\subsubsection{The SHERPA Project - Collaborative Mission}

The robotic manipulator presented in this thesis was designed and built in the scope of the SHERPA project. As such, many practical engineering problems were solved in order to facilitate the mission. The design, construction, and integration of the arm, as described in Chapters 3, 4, and 6, addressed the engineering objectives and demonstrated the viability of mechanically compliant manipulation for autonomous interaction and servicing tasks. The manipulator has shown to be able to demonstrate the required tasks in an outdoor environment, however its development is closer to an academic than industrial level in terms of product maturity.

Chapter 6 also answered the research objective RO5 through demonstrating the planning and execution of complex collaborative missions applied to a mountain rescue scenario. The proposed mission includes a number of different agents, with various sets of capabilities and varying availability, including human rescuers, smalland large-scale UAVs, and mobile ground robots. The challenge of specifying and planning tasks among heterogeneous agents, that furthermore may already be occupied with other assignments, or have other resource restrictions, was approached by formulating high-level control operations as task specific trees, a hierarchical task description that can integrate such heterogeneous agents and several layers of abstraction. The SHERPA delegation framework uses an auctioning system to determine the most suited agent to execute each node of the task specific tree, representing sub-tasks of the mission goal, according to the constraint problem which takes the mission requirements and platform capabilities into account. The control and coordination of multiple agents was demonstrated by replacing the battery of a landed UAV in an outdoor setting, involving a UAV, and the ground rover equipped with the compliant manipulator and UAV service station. While this demonstration did not yet simulate a complete and sustained autonomous mission due to the limited duration and scope of the mission, it did verify that all necessary components are in place.

\subsection{Future Work}

The main motivation behind this research is to design and build a compliant robotic manipulator that can support mountain rescue operations. The work presented in this thesis already shows the usefulness of the developed system, however, the manipulator 
is not ready to be directly applied in the field, and more steps are needed in order for the SHERPA system to reach a sufficient maturity.

On the level of the compliant actuators and joints the following engineering and research points may be addressed:

1. The control setup current used only allows to control the position and velocities of the actuators. A fast and accurate torque control is required for properly controlling the impedance of the actuators, for instance through active impedance and inertia shaping.

2. Parasitic compliance introduced to the joints for instance through tendons should be removed by reinforcing the transmission, and replaced instead by reliable and properly dimensioned series elastic elements designed according to the methodology presented in Chapter 2 where compliance is required.

3. Additional research may be carried out into the polymer springs' mechanical properties, such as creep resistance and fatigue. A wider range of materials and production methods, such as shape deposition manufacturing, should also be investigated to improve performance, and ease and flexibility of production.

4. Mechanically coupling the compliance of the two distal wrist joints has shown that actuators and mechanism can be combined in multi-dimensional VSAs. Depending on the actuation topology, such mechanisms can reduce the mass, volume, and number of actuators of the system, and may be investigated further.

More work also may be done at the level of compliant manipulators:

1. The manipulator's actuation topology so far has only been analyzed empirically. Chapter 5 presents tools to approach this problem in an analytic and quantitative manner. After testing and revising the core tasks of the arm, the actuation topology should be optimized with respect to the proposed metric.

2. Likewise, the compliant control of the manipulator may be implemented according to the findings of Chapter 5 and utilize the proposed projectors to divide tasks in constrained and elastic motions and forces and to properly exploit the arm's mechanical compliance.

3. Up to now only the mechanical compliance of the manipulator has been controlled. This may be combined with an active Cartesian impedance controller in order to realize compliant behavior that cannot be achieved through the mechanical structure alone.

4. The controlled passive dynamics of the manipulator can be used to absorb impacts, and for instance catch a moving UAV from the air. The passive dynamics can also be exploited in cyclic or explosive tasks, for instance launching a UAV by throwing it into the air. In this way the passive compliance contributes to these tasks by storing and releasing energy, and absorbing impacts. 
In order to further develop the SHERPA platform, the following steps may be carried out:

1. More engineering work is required in order to increase the SHERPA platform's reliability, robustness, and ease of use for the end user. This includes increasing the mechanical robustness and improving low and mid level software and control framework of the manipulator.

2. The next benchmark of the system should be continuous flight of the UAVs, supported by the manipulator and service station. Achieving this goal would demonstrate the advances required to bring the platform closer to its final application. 


\section{BIBLIOGRAPHY}

[1] E. Garcia, M. A. Jimenez, P. G. D. Santos, and M. Armada, "The evolution of robotics research," IEEE Robotics Automation Magazine, vol. 14, no. 1, pp. 90-103, March 2007.

[2] L. Marconi, F. Basile, G. Caprari, R. Carloni, P. Chiacchio, C. Hurzeler, V. Lippiello, R. Naldi, J. Nikolic, B. Siciliano, S. Stramigioli, and E. Zwicker, "Aerial service robotics: The airobots perspective," in 2012 2nd International Conference on Applied Robotics for the Power Industry (CARPI), Sept 2012, pp. 64-69.

[3] J. Casper and R. R. Murphy, "Human-robot interactions during the robotassisted urban search and rescue response at the world trade center," IEEE Transactions on Systems, Man, and Cybernetics, Part B (Cybernetics), vol. 33, no. 3, pp. 367-385, June 2003.

[4] K. Nagatani, S. Kiribayashi, Y. Okada, K. Otake, K. Yoshida, S. Tadokoro, T. Nishimura, T. Yoshida, E. Koyanagi, M. Fukushima, and S. Kawatsuma, "Emergency response to the nuclear accident at the fukushima daiichi nuclear power plants using mobile rescue robots," Journal of Field Robotics, vol. 30, no. 1, pp. 44-63, 2013.

[5] R. Penna, W. Allasia, L. Bianchi, E. Licata, P. Duranti, A. Molino, E. Bagalini, S. Sagliocco, S. Scarafia, P. Prinetto, G. Airofarulla, and A. Carelli, "Salveremo, an automatic system for the search and rescue in the wilderness and mountain areas," pp. EGU2016-6192-1, 042016.

[6] T. B. Sheridan, "Human-robot interaction: Status and challenges," Human Factors, vol. 58, no. 4, pp. 525-532, 2016.

[7] Österreichischer Bergrettungsdienst Bundesverband, "Jahresbericht 2016," URL: www.bergrettung.at/wp-content/uploads/OeBRD_Jahresbericht_2016. pdf [in German]. 
[8] Schweizer Alpen-Club SAC, "Bergnotfälle Schweiz," URL: www.sac-cas. ch/unterwegs/sicherheit/bergnotfallstatistik.html [Available in German and French]. accessed 2018-01-01.

[9] Swiss Air-Rescue, "Rega missions 2001 - 2016," URL: www.rega.ch/pdf/ medien/2017/Einsatzstatistik_2016_en.pdf.

[10] A. D. Santis, B. Siciliano, A. D. Luca, and A. Bicchi, "An atlas of physical human-robot interaction," Mechanism and Machine Theory, vol. 43, no. 3, pp. $253-270,2008$.

[11] S. Haddadin, S. Parusel, R. Belder, and A. Albu-Schäffer, It Is (Almost) All about Human Safety: A Novel Paradigm for Robot Design, Control, and Planning. Berlin, Heidelberg: Springer Berlin Heidelberg, 2013, pp. 202-215.

[12] L. Marconi et al., "The SHERPA project: Smart collaboration between humans and ground-aerial robots for improving rescuing activities in alpine environments," in Proceedings of the IEEE International Symposium on Safety, Security, and Rescue Robotics, 2012, pp. 1-4.

[13] J. Cacace, A. Finzi, and V. Lippiello, "Multimodal interaction with multiple co-located drones in search and rescue missions," CoRR, vol. abs/1605.07316, 2016.

[14] Alpine Rettung Schweiz, "Drohnen in der Bergrettung," Der Bergretter. URL: www.alpinerettung.ch/portrait/publikationen/der-bergretter. Accessed 201801-1. [Available in German, French, and Italian], pp. 4-6, Mai 2016.

[15] Bergwacht Bayern, "70-jährigen Wanderer nach intensiver Suche mittels Drohne gefunden," Jahresbericht 2017. URL: www.bergwacht-bayern.de/ uploads/media/Jahresbericht_2017_gesamt_01.pdf [in German], p. 27.

[16] AslaTech P.I., Bologna, Italy. Website. URL: www.aslatech.com. Accessed 2018-01-15.

[17] BlueBotics SA, St-Sulpice, Switzerland. Website. URL: www.bluebotics.com. 2016. Accessed 2018-01-15.

[18] Autonomous Systems Lab, ETH Zürich, "senseSoar," Website. URL: www. sensesoar.ethz.ch. 2011. Accessed 2018-01-15.

[19] A. Sato, “The RMAX Helicopter UAV,” Public report. Aeronautic Operations YAMAHA MOTOR CO., LTD., Shizuoka, Japan, 2003.

[20] P. Haegeli, M. Falk, H. Brugger, H. Etter, and J. Boyd, "Comparison of avalanche survival patterns in canada and switzerland," Canadian Medical Association Journal, vol. 183(7), pp. 789-795, 2011. 
[21] H. Brugger, H. J. Etter, B. Zweifel, P. Mair, M. Hohlrieder, J. Ellerton, F. Elsensohn, J. Boyd, G. Sumann, and M. Falk, "The impact of avalanche rescue devices on survival," Resuscitation, vol. 75, no. 3, pp. 476 - 483, 2007.

[22] C. K. Grissom, "Lessons learned from avalanche survival patterns," Canadian Medical Association Journal, vol. 183, no. 7, pp. E366-E367, 2011. [Online]. Available: http://www.cmaj.ca/content/183/7/E366

[23] E. Procter, G. Strapazzon, T. D. Cappello, B. Zweifel, A. Wrtele, A. Renner, M. Falk, and H. Brugger, "Burial duration, depth and air pocket explain avalanche survival patterns in austria and switzerland," Resuscitation, vol. 105, no. Supplement C, pp. 173 - 176, 2016.

[24] F. Techel and B. Zweifel, "Recreational avalanche accidents in Switzerland: Trends and patterns with an emphasis on burial, rescue methods and avalanche danger," in Proceedings of the International Snow Science Workshop, 2013, pp. 1106-1112.

[25] M. Bejiga, A. Zeggada, A. Nouffidj, and F. Melgani, "A convolutional neural network approach for assisting avalanche search and rescue operations with uav imagery," Remote Sensing, vol. 9, p. 100, 012017.

[26] F. Fruehauf, A. Heilig, M. Schneebeli, W. Fellin, and O. Scherzer, "Experiments and algorithms to detect snow avalanche victims using airborne groundpenetrating radar," IEEE Transactions on Geoscience and Remote Sensing, vol. 47, no. 7, pp. 2240-2251, July 2009.

[27] Team Alcedo, ETH Zürich, "Alcedo - The Flying Avalanche Transceiver," Website. www.alcedo.ethz.ch/. 2010. Accessed 2018-01-01.

[28] M. Silvagni, A. Tonoli, E. Zenerino, and M. Chiaberge, "Multipurpose uav for search and rescue operations in mountain avalanche events," Geomatics, Natural Hazards and Risk, vol. 8, no. 1, pp. 18-33, 2017.

[29] Bergrettungsdienst im Alpenverein Südtirol Landesverband/Soccorso Alpino dell'Alpenverein Südtirol Unione Provinciale, "Jahresbericht 2016," URL: http://www.bergrettung.it/en/jahresberichte.html [in German].

[30] Z. Bien, D.-J. Kim, M.-J. Chung, D.-S. Kwon, and P.-H. Chang, "Development of a wheelchair-based rehabilitation robotic system (kares ii) with various human-robot interaction interfaces for the disabled," in Proceedings 2003 IEEE/ASME International Conference on Advanced Intelligent Mechatronics (AIM 2003), vol. 2, July 2003, pp. 902-907 vol.2.

[31] S. Groothuis, S. Stramigioli, and R. Carloni, "Lending a helping hand," IEEE Robotics and Automation Magazine, vol. 20, no. 1, pp. 20-29, 2013. 
[32] K. Kong, J. Bae, and M. Tomizuka, "Control of rotary series elastic actuator for ideal force-mode actuation in human-robot interaction applications," IEEE/ASME Transactions on Mechatronics, vol. 14, pp. 105-118, Feb 2009.

[33] H. Vallery, J. Veneman, E. van Asseldonk, R. Ekkelenkamp, M. Buss, and H. van Der Kooij, "Compliant actuation of rehabilitation robots," IEEE Robotics Automation Magazine, vol. 15, no. 3, pp. 60-69, September 2008.

[34] P. Beyl, K. Knaepen, S. Duerinck, M. V. Damme, B. Vanderborght, R. Meeusen, and D. Lefeber, "Safe and compliant guidance by a powered knee exoskeleton for robot-assisted rehabilitation of gait," Advanced Robotics, vol. 25, no. 5, pp. 513-535, 2011.

[35] M. Hutter, C. Gehring, M. Bloesch, M. A. Hoepflinger, C. D. Remy, and R. Siegwart, "Starleth: A compliant quadrupedal robot for fast, efficient, and versatile locomotion," in Adaptive Mobile Robotics, 2012, pp. 483-490.

[36] T. Boaventura, C. Semini, J. Buchli, M. Frigerio, M. Focchi, and D. G. Caldwell, "Dynamic torque control of a hydraulic quadruped robot," in 2012 IEEE International Conference on Robotics and Automation, May 2012, pp. 18891894.

[37] B. Vanderborght, B. Verrelst, R. V. Ham, M. V. Damme, D. Lefeber, B. M. Y. Duran, and P. Beyl, "Exploiting natural dynamics to reduce energy consumption by controlling the compliance of soft actuators," The International Journal of Robotics Research, vol. 25, no. 4, pp. 343-358, 2006.

[38] D. Leidner, C. Borst, A. Dietrich, M. Beetz, and A. Albu-Schffer, "Classifying compliant manipulation tasks for automated planning in robotics," in 2015 IEEE/RSJ International Conference on Intelligent Robots and Systems (IROS), Sept 2015, pp. 1769-1776.

[39] M. Jonsson, A. Stolt, A. Robertsson, S. von Gegerfelt, and K. Nilsson, "On force control for assembly and deburring of castings," Production Engineering, vol. 7, no. 4, pp. 351-360, Jul 2013.

[40] A. Stolt, M. Linderoth, A. Robertsson, and R. Johansson, "Force controlled robotic assembly without a force sensor," in 2012 IEEE International Conference on Robotics and Automation, May 2012, pp. 1538-1543.

[41] G. A. Pratt and M. M. Williamson, "Series elastic actuators," in Proceedings of the IEEE/RSJ International Conference on Intelligent Robots and Systems, 1995, pp. 399-406.

[42] B. Vanderborght et al., "Variable impedance actuators: A review," Robotics and Autonomous Systems, vol. 61, no. 12, pp. 1601-1614, 2013. 
[43] S. Wolf et al., "Variable stiffness actuators: Review on design and components," IEEE/ASME Transactions on Mechatronics, vol. 21, no. 5, pp. 24182430, 2016.

[44] A. Bicchi, G. Tonietti, M. Bavaro, and M. Piccigallo, "Variable stiffness actuators for fast and safe motion control," vol. 15, pp. 527-536, 012003.

[45] M. Laffranchi, N. G. Tsagarakis, and D. G. Caldwell, "Safe human robot interaction via energy regulation control," in 2009 IEEE/RSJ International Conference on Intelligent Robots and Systems, Oct 2009, pp. 35-41.

[46] F. Negrello, M. Garabini, M. G. Catalano, J. Malzahn, D. G. Caldwell, A. Bicchi, and N. G. Tsagarakis, "A modular compliant actuator for emerging high performance and fall-resilient humanoids," in 2015 IEEE-RAS 15th International Conference on Humanoid Robots (Humanoids), Nov 2015, pp. 414-420.

[47] A. Calanca and P. Fiorini, On the Role of Compliance in Force Control. Cham: Springer International Publishing, 2016, pp. 1243-1255. [Online]. Available: https://doi.org/10.1007/978-3-319-08338-4_90

[48] J. Pratt, B. Krupp, and C. Morse, "Series elastic actuators for high fidelity force control," Industrial Robot, vol. 29, no. 3, pp. 234-241, 2002. [Online]. Available: https://doi.org/10.1108/01439910210425522

[49] J. W. Sensinger and R. F. F. Weir, "Improvements to series elastic actuators," in 2006 2nd IEEE/ASME International Conference on Mechatronics and Embedded Systems and Applications, Aug 2006, pp. 1-7.

[50] D. Paluska and H. Herr, "The effect of series elasticity on actuator power and work output: Implications for robotic and prosthetic joint design," Robotics and Autonomous Systems, vol. 54, no. 8, pp. 667 - 673, 2006.

[51] M. Garabini, A. Passaglia, F. Belo, P. Salaris, and A. Bicchi, "Optimality principles in stiffness control: The vsa kick,” pp. 3341-3346, 052012.

[52] B. Vanderborght, N. G. Tsagarakis, C. Semini, R. V. Ham, and D. G. Caldwell, "Maccepa 2.0: Adjustable compliant actuator with stiffening characteristic for energy efficient hopping," in 2009 IEEE International Conference on Robotics and Automation, May 2009, pp. 544-549.

[53] A. Jafari, N. G. Tsagarakis, and D. G. Caldwell, "Exploiting natural dynamics for energy minimization using an actuator with adjustable stiffness (awas)," in 2011 IEEE International Conference on Robotics and Automation, May 2011, pp. 4632-4637. 
[54] J. Fryman and B. Matthias, "Safety of industrial robots: From conventional to collaborative applications," in ROBOTIK 2012; 7th German Conference on Robotics, May 2012, pp. 1-5.

[55] Transparency Market Research, "Collaborative Robot Market - Global Industry Analysis, Size, Share, Growth, Trends and Forecast 2016 - 2024, 2017,’ Report. URL: $\quad$ www.transparencymarketresearch.com/collaborative-robots-market. html. 2017-01-31. Accessed 2018-01-15.

[56] Universal Robots A/S, "The future is collaborative," Product brochure. URL: www.universal-robots.com. Accessed 2018-01-15, Sep. 2016.

[57] Rethink Robotics, "Sawyer - Smart, Collaborative Difference," Product datasheet. URL: www.rethinkrobotics.com. Accessed 2018-01-15, Apr. 2017.

[58] — - "Baxter - Redefining Automation," Product datasheet. URL: www. rethinkrobotics.com. Accessed 2018-01-15, Oct. 2015.

[59] Kinova Robotics, "JACO ${ }^{2}$ - Assistive user guide," User guide. URL: www. kinovarobotics.com. Accessed 2018-01-15, 2017.

[60] FANUC Europe Corporation, "CR series - Powerful collaborative robots for a wide range of applications," Brochure. URL: www.fanuc.eu. Accessed 201801-15, 2017.

[61] G. Hirzinger, N. Sporer, A. Albu-Schaffer, M. Hahnle, R. Krenn, A. Pascucci, and M. Schedl, "DLR's torque-controlled light weight robot III-are we reaching the technological limits now?" in Proceedings 2002 IEEE International Conference on Robotics and Automation, vol. 2, 2002, pp. 1710-1716.

[62] E. Guizzo, "Franka: A robot arm that's safe, low cost, and can replicate itself," IEEE Spectrum, vol. 54, no. 1, pp. 34-35, January 2017.

[63] ABB Engineering (Shanghai) Ltd., "YuMi ${ }^{\circledR}$ creating an automated future together," Product datasheet. URL: www.abb.com/robotics. Accessed 2018-0115, Sep. 2016.

[64] B. Rooks, “The harmonious robot," Industrial robot: An International Journal, vol. 33, pp. 125-130, 032006.

[65] F\&P Robotics AG, "P-Rob ${ }^{\circledR} 2 \mathrm{R}$ - 6-axis collaborative and lightweight robot arm," Technical specifications. URL: www.fp-robotics.com. Accessed 201801-15, Dec. 2017.

[66] Robert Bosch Manufacturing Solutions GmbH, “APAS - Intelligent Systems for Human-Machine Collaboration," Product brochure. URL: www.bosch-apas. com. Accessed 2018-01-15, Mar. 2017. 
[67] Yaskawa America, Inc. - Motoman Robotics Division, "HC10 Human-Collaborative Robot," Product datasheet. URL: www.motoman.com/ collaborative. Accessed 2018-01-15, Oct. 2017.

[68] P. Francesco and G. G. Paolo, "Aura: An example of collaborative robot for automotive and general industry applications," Procedia Manufacturing, vol. 11, pp. 338 - 345, 2017, 27th International Conference on Flexible Automation and Intelligent Manufacturing, FAIM2017, 27-30 June 2017, Modena, Italy.

[69] Stäubli International AG, "TX2-40 industrial robot," Product leaflet. URL: www.staubli.com/robotics. Jun. 2016. Accessed 2018-01-15.

[70] M. Wassink and S. Stramigioli, "Towards a novel safety norm for domestic robotics," in 2007 IEEE/RSJ International Conference on Intelligent Robots and Systems, Oct 2007, pp. 3354-3359.

[71] "ISO 10218 - Robots and robotic devices - Safety requirements for industrial robots," International Organization for Standardization, Geneva, CH, Standard, Jul. 2011.

[72] "ISO/TS 15066 - Robots and robotic devices - Collaborative robots," International Organization for Standardization, Geneva, CH, Tech. Rep., Feb. 2016.

[73] P. Maiolino, M. Maggiali, G. Cannata, G. Metta, and L. Natale, "A flexible and robust large scale capacitive tactile system for robots," IEEE Sensors Journal, vol. 13, no. 10, pp. 3910-3917, Oct 2013.

[74] M. Kalakrishnan, L. Righetti, P. Pastor, and S. Schaal, "Learning force control policies for compliant manipulation," in 2011 IEEE/RSJ International Conference on Intelligent Robots and Systems, Sept 2011, pp. 4639-4644.

[75] N. Paine, J. S. Mehling, J. Holley, N. Radford, G. Johnson, C. Fok, and L. Sentis, "Actuator control for the NASA-JSC valkyrie humanoid robot: A decoupled dynamics approach for torque control of series elastic robots," vol. 32, 052015.

[76] N. Tsagarakis et al., "Walk-man: A high performance humanoid platform for realistic environments," Journal of Field Robotics (JFR), vol. 34, p. 1225?1259, 2017.

[77] L. Baccelliere, N. Kashiri, L. Muratore, A. Laurenzi, M. Kamedula, A. Margan, S. Cordasco, J. Malzahn, and N. G. Tsagarakis, "Development of a human size and strength compliant bi-manual platform for realistic heavy manipulation tasks," in 2017 IEEE/RSJ International Conference on Intelligent Robots and Systems (IROS), Sept 2017, pp. 5594-5601. 
[78] T. Morita and S. Sugano, "Development and evaluation of seven dof mia arm," in Proceedings of the IEEE International Conference on Robotics and Automation, vol. 1, 1997, pp. 462-467.

[79] M. Grebenstein, A. Albu-Schffer, T. Bahls, M. Chalon, O. Eiberger, W. Friedl, R. Gruber, S. Haddadin, U. Hagn, R. Haslinger, H. Hppner, S. Jrg, M. Nickl, A. Nothhelfer, F. Petit, J. Reill, N. Seitz, T. Wimbck, S. Wolf, T. Wsthoff, and G. Hirzinger, "The dlr hand arm system," in 2011 IEEE International Conference on Robotics and Automation, May 2011, pp. 3175-3182.

[80] S. Haddadin, F. Huber, and A. Albu-Schffer, "Optimal control for exploiting the natural dynamics of variable stiffness robots," in 2012 IEEE International Conference on Robotics and Automation, May 2012, pp. 3347-3354.

[81] M. Garabini, A. Passaglia, F. Belo, P. Salaris, and A. Bicchi, "Optimality principles in variable stiffness control: The vsa hammer," in 2011 IEEE/RSJ International Conference on Intelligent Robots and Systems, Sept 2011, pp. 37703775 .

[82] E. Barrett, M. Fumagalli, and R. Carloni, "Elastic energy storage in leaf springs for a lever-arm based variable stiffness actuator," in Proceedings of the IEEE/RSJ International Conference on Intelligent Robots and Systems, 2016.

[83] E. Barrett, M. Reiling, G. Barbieri, M. Fumagalli, and R. Carloni, "Mechatronic design of a variable stiffness robotic arm," in Proceedings of the IEEE/RSJ International Conference on Intelligent Robots and Systems, 2017, pp. 45824588 .

[84] E. Barrett, M. Reiling, S. Mirhassani, R. Meijering, J. Jager, N. Mimmo, F. Callegati, L. Marconi, R. Carloni, and S. Stramigioli, "Autonomous battery exchange of uavs with a mobile ground base," in Proceedings of the IEEE International Conference on Robotics and Automation, 2018.

[85] E. Barrett and M. Fumagalli and R. Carloni, "The SHERPA gripper: Grasping of small-scale uavs," in Proceedings of the IEEE International Symposium on Safety, Security and Rescue Robotics, 2016, pp. 384-389.

[86] B. Vanderborght et al., "Variable impedance actuators: Moving the robots of tomorrow," in Proceedings of the IEEE/RSJ International Conference on Intelligent Robots and Systems, 2012, pp. 5454-5455.

[87] R. Pfeifer and J. Bongard, How the body shapes the way we think: a new view of intelligence. MIT press, 2006.

[88] G. Grioli et al., "Variable impedance actuators: The user's point of view," International Journal of Robotic Research, vol. 34, no. 6, pp. 727-743, 2015. 
[89] S. Wolf, O. Eiberger, and G. Hirzinger, "The DLR FSJ: Energy based design of a variable stiffness joint," in Proceedings of the IEEE International Conference on Robotics and Automation, 2011, pp. 5082-5089.

[90] R. V. Ham, B. Vanderborght, M. V. Damme, B. Verrelst, and D. Lefeber, "MACCEPA, the mechanically adjustable compliance and controllable equilibrium position actuator: Design and implementation in a biped robot," Robotics and Autonomous Systems, vol. 55, no. 10, pp. 761 - 768, 2007.

[91] S. Groothuis, G. Rusticelli, A. Zucchelli, S. Stramigioli, and R. Carloni, "The vsaUT-II: a novel rotational variable stiffness actuator," in Proceedings of the IEEE International Conference on Robotics and Automation, 2012, pp. 33553360.

[92] N. Tsagarakis, I. Sardellitti, and D. Caldwell, "A new variable stiffness actuator (CompAct-VSA): Design and modelling," in Proceedings of the IEEE/RSJ International Conference on Intelligent Robots and Systems, 2011, pp. 378-383.

[93] A. Jafari, N. Tsagarakis, and D. Caldwell, "AwAS-II: A new actuator with adjustable stiffness based on the novel principle of adaptable pivot point and variable lever ratio," in Proceedings of the IEEE International Conference on Robotics and Automation, 2011, pp. 4638-4643.

[94] J. Choi, S. Hong, W. Lee, S. Kang, and M. Kim, "A robot joint with variable stiffness using leaf springs," IEEE Transactions on Robotics, vol. 27, no. 2, pp. 229-238, April 2011.

[95] F. Negrello, M. Catalano, M. Garabini, M. Poggiani, D. G. Caldwell, N. Tsagarakis, and A. Bicchi, "Design and characterization of a novel highcompliance spring for robots with soft joints," 2017 IEEE International Conference on Advanced Intelligent Mechatronics (AIM), pp. 271-278, 2017.

[96] G. Grioli et al., "Variable stiffness actuators: The user's point of view," International Journal of Robotics Research, vol. 34, no. 6, pp. 727-743, 2015.

[97] B. Verrelst, R. V. Ham, B. Vanderborght, D. Lefeber, F. Daerden, and M. V. Damme, "Second generation pleated pneumatic artificial muscle and its robotic applications," Advanced Robotics, vol. 20, no. 7, pp. 783-805, 2006.

[98] G. Tonietti, R. Schiavi, and A. Bicchi, "Design and control of a variable stiffness actuator for safe and fast physical human/robot interaction," in Proceedings of the 2005 IEEE International Conference on Robotics and Automation, April 2005, pp. 526-531. 
[99] R. Schiavi, G. Grioli, S. Sen, and A. Bicchi, "Vsa-ii: a novel prototype of variable stiffness actuator for safe and performing robots interacting with humans," in 2008 IEEE International Conference on Robotics and Automation, May 2008, pp. 2171-2176.

[100] O. Eiberger, S. Haddadin, M. Weis, A. Albu-Schffer, and G. Hirzinger, "On joint design with intrinsic variable compliance: derivation of the dlr qa-joint," in 2010 IEEE International Conference on Robotics and Automation, May 2010, pp. 1687-1694.

[101] A. Jafari, N. G. Tsagarakis, and D. G. Caldwell, "A novel intrinsically energy efficient actuator with adjustable stiffness (awas)," IEEE/ASME Transactions on Mechatronics, vol. 18, no. 1, pp. 355-365, Feb 2013.

[102] B. S. Kim and J. B. Song, "Hybrid dual actuator unit: A design of a variable stiffness actuator based on an adjustable moment arm mechanism," in 2010 IEEE International Conference on Robotics and Automation, May 2010, pp. $1655-1660$.

[103] H. V. Quy, L. Aryananda, F. I. Sheikh, F. Casanova, and R. Pfeifer, "A novel mechanism for varying stiffness via changing transmission angle," in 2011 IEEE International Conference on Robotics and Automation, May 2011, pp. 5076-5081.

[104] S. S. Groothuis, G. Rusticelli, A. Zucchelli, S. Stramigioli, and R. Carloni, "The vsaUT-II: A novel rotational variable stiffness actuator," in Proceedings of the IEEE International Conference on Robotics and Automation, 2012, pp. 3355-3360.

[105] M. Fumagalli, E. Barrett, S. Stramigioli, and R. Carloni, "The mvsa-ut: A miniaturized differential mechanism for a continuous rotational variable stiffness actuator," in 2012 4th IEEE RAS EMBS International Conference on Biomedical Robotics and Biomechatronics (BioRob), June 2012, pp. 19431948.

[106] S. Groothuis, R. Carloni, and S. Stramigioli, "A novel variable stiffness mechanism capable of an infinite stiffness range and unlimited decoupled output motion," in Actuators, vol. 3, no. 2. Multidisciplinary Digital Publishing Institute, 2014, pp. 107-123.

[107] K. W. Hollander, T. G. Sugar, and D. E. Herring, "Adjustable robotic tendon using a 'jack spring' trade;," in 9th International Conference on Rehabilitation Robotics, 2005. ICORR 2005., June 2005, pp. 113-118.

[108] A. van der Schaft, Port-Hamiltonian systems: an introductory survey. European Mathematical Society Publishing House (EMS Ph), 2006, no. suppl 2, pp. 1339-1365. 
[109] P. C. Breedveld, Modeling and simulation of dynamic systems using bond graphs. Oxford, UK: Eolss Publishers, 2008.

[110] H. Paynter, "Analysis and design of engineering systems; class notes for mit course 2,751..” 1961.

[111] P. Breedveld, "Physical systems theory in terms of bond graphs /," 011984.

[112] J. Cervera, A. van der Schaft, and A. Baos, "Interconnection of porthamiltonian systems and composition of dirac structures," Automatica, vol. 43, no. 2 , pp. $212-225,2007$.

[113] A. van der Schaft and D. Jeltsema, "Port-hamiltonian systems theory: An introductory overview," Foundations and Trends in Systems and Control, vol. 1, no. 2-3, pp. 173-378, 2014.

[114] L. C. Visser, R. Carloni, and S. Stramigioli, "Energy-efficient variable stiffness actuators," IEEE Transactions on Robotics, vol. 27, no. 5, pp. 865-875, 2011.

[115] S. Wolf and G. Hirzinger, "A new variable stiffness design: Matching requirements of the next robot generation," in 2008 IEEE International Conference on Robotics and Automation, May 2008, pp. 1741-1746.

[116] R. Carloni, L. C. Visser, and S. Stramigioli, "Variable stiffness actuators: A port-based power-flow analysis," IEEE Transactions on Robotics, vol. 28, no. 1, pp. 1-11, Feb 2012.

[117] L. C. Visser, R. Carloni, and S. Stramigioli, "Variable stiffness actuators: A port-based analysis and a comparison of energy efficiency," in 2010 IEEE International Conference on Robotics and Automation, May 2010, pp. 3279-3284.

[118] — - "Energy-efficient variable stiffness actuators," IEEE Transactions on Robotics, vol. 27, no. 5, pp. 865-875, 2011.

[119] J. Collins, H. Busby, and G. Staab, Mechanical Design of Machine Elements and Machines. Wiley, 2010.

[120] J. Grote, K.-H. and. Feldhusen, Dubbel. Springer Berlin Heidelberg, 2007.

[121] H. Wittel, H. Roloff, and W. Matek, Roloff/Matek Maschinenelemente: Tabellenbuch, ser. Viewegs Fachbücher der Technik. Vieweg \& Teubner, 2009.

[122] Ensinger GmbH, Material datasheets. URL: http://www.ensinger-online.com/ en/materials/.

[123] M. Ashby, Materials Selection in Mechanical Design, 3rd ed. ButterworthHeinemann, 2005. 
[124] Y. Yamada and T.Kuwabara, Materials for Springs. Berlin: Springer, 2007.

[125] L. T. Martins, C. Tatsch, E. H. Maciel, R. V. B. Henriques, R. Gerndt, and R. S. da Guerra, Polyurethane-Based Modular Series Elastic Upgrade to a Robotics Actuator. Springer International Publishing, 2015, pp. 347-355.

[126] R. R. Ma, L. U. Odhner, and A. M. Dollar, "A modular, open-source 3d printed underactuated hand," in 2013 IEEE International Conference on Robotics and Automation, May 2013, pp. 2737-2743.

[127] A. Albu-Schaffer, O. Eiberger, M. Grebenstein, S. Haddadin, C. Ott, T. Wimbock, S. Wolf, and G. Hirzinger, "Soft robotics," IEEE Robotics Automation Magazine, vol. 15, no. 3, pp. 20-30, September 2008.

[128] A. Albu-Schaffer, C. Ott, U. Frese, and G. Hirzinger, "Cartesian impedance control of redundant robots: recent results with the dlr-light-weight-arms," in 2003 IEEE International Conference on Robotics and Automation (Cat. No.03CH37422), vol. 3, Sept 2003, pp. 3704-3709 vol.3.

[129] Y. Cao, K. Lu, X. Li, and Y. Zang, "Accurate numerical methods for computing $2 \mathrm{~d}$ and $3 \mathrm{~d}$ robot workspace," International Journal of Advanced Robotic Systems, vol. 8, no. 6, p. 76, 2011.

[130] P. Chiacchio and B. Siciliano, "A closed-loop jacobian transpose scheme for solving the inverse kinematics of nonredundant and redundant wrists," vol. 6, pp. $601-630,101989$.

[131] B. Siciliano, L. Sciavicco, L. Villani, and G. Oriolo, Robotics: Modelling, Planning and Control, 1st ed. Springer Publishing Company, Incorporated, 2008.

[132] S. S. Groothuis, S. Stramigioli, and R. Carloni, "Modeling robotic manipulators powered by variable stiffness actuators: A graph-theoretic and port-hamiltonian formalism," IEEE Transactions on Robotics, vol. 33, no. 4, pp. 1-12, 2017.

[133] _ _ "Compliant robotic systems on graphs," in Proceedings of the IEEE/RSJ International Conference on Intelligent Robots and Systems, 2014, pp. 38983903.

[134] TQ Systems GmbH, "Servo kits ILM," Product information. URL: www. robodrive.com, 2016.

[135] Harmonic Drive AG, “CSD-2A Component Sets," Engineering Data. URL: www.harmonicdrive.de/3060, Nov. 2011.

[136] maxon motor ag, "Program 2017/18 - High Precision Drives and Systems," Product catalog. URL: epaper.maxonmotor.com, May 2017. 
[137] D. Tan, D. Brouwer, M. Fumagalli, and R. Carloni, "A 2-DOF joint with coupled variable output stiffness," IEEE Robotics and Automation Letters, vol. 2, no. 1, pp. 366-372, 2017.

[138] ATI Industrial Automation, "F/T Transducer - Six-Axis Force/Torque Sensor System,” Installation and operation manual. URL: www.ati-ia.com, Mar. 2016.

[139] ams AG, “AS5048A/AS5048B - Magnetic Rotary Encoder (14-Bit Angular Position Sensor)," Product datasheet. URL: www.ams.com, Nov. 2015.

[140] Elmo Motion Control Ltd., "Solo Whistle Digital Servo Drive Installation Guide," Product installation guide. URL: www.elmomc.com, Oct. 2017.

[141] M. Quigley, K. Conley, B. Gerkey, J. Faust, T. Foote, J. Leibs, R. Wheeler, and A. Y. Ng, "Ros: an open-source robot operating system," in ICRA workshop on open source software, vol. 3, no. 3.2, 2009, p. 5.

[142] “MoveIt! motion planning framework," http://moveit.ros.org/, accessed: 201008-01.

[143] I. A. Şucan, M. Moll, and L. E. Kavraki, "The Open Motion Planning Library," IEEE Robotics \& Automation Magazine, vol. 19, no. 4, pp. 72-82, 2012.

[144] R. Deimel and O. Brock, "A novel type of compliant, underactuated robotic hand for dexterous grasping," in Proceedings of Robotics: Science and Systems, Berkeley, USA, July 2014.

[145] C. Melchiorri, G. Palli, G. Berselli, and G. Vassura, "Development of the ub hand iv: Overview of design solutions and enabling technologies," Robotics Automation Magazine, IEEE, vol. 20, no. 3, pp. 72-81, Sept 2013.

[146] M. Ciocarlie, F. M. Hicks, R. Holmberg, J. Hawke, M. Schlicht, J. Gee, S. Stanford, and R. Bahadur, "The velo gripper: A versatile single-actuator design for enveloping, parallel and fingertip grasps," The International Journal of Robotics Research, vol. 33, no. 5, pp. 753-767, 2014.

[147] M. Grebenstein, M. Chalon, W. Friedl, S. Haddadin, T. Wimbck, G. Hirzinger, and R. Siegwart, "The hand of the DLR hand arm system: Designed for interaction," The International Journal of Robotics Research, vol. 31, no. 13, pp. 1531-1555, 2012.

[148] M. Fumagalli, E. Barrett, S. Stramigioli, and R. Carloni, "Analysis of an underactuated robotic finger with variable pinch and closure grasp stiffness," in Proceedings of the IEEE International Conference on Advanced Intelligent Mechatronics, 2016.

[149] RobotiQ, http:www.robotiq.com/. 
[150] Barrett Technology Inc., “The BarrettHand ${ }^{T M}$.” http://www.barrett.com/robot/ products-hand.htm.

[151] F. Petit and A. Albu-Schffer, "Cartesian impedance control for a variable stiffness robot arm," in Proceedings of the IEEE/RSJ International Conference on Intelligent Robots and Systems, 2011, pp. 4180-4186.

[152] S. Groothuis, "On the modeling, design, and control of compliant robotic manipulators," 62016.

[153] M. Mosadeghzad, G. A. Medrano-Cerda, J. A. Saglia, N. G. Tsagarakis, and D. G. Caldwell, "Comparison of various active impedance control approaches, modeling, implementation, passivity, stability and trade-offs," in 2012 IEEE/ASME International Conference on Advanced Intelligent Mechatronics (AIM), July 2012, pp. 342-348.

[154] Z. Li, B. Vanderborght, N. G. Tsagarakis, L. Colasanto, and D. G. Caldwell, "Stabilization for the compliant humanoid robot coman exploiting intrinsic and controlled compliance," in 2012 IEEE International Conference on Robotics and Automation, May 2012, pp. 2000-2006.

[155] A. Albu-Schffer, C. Ott, and G. Hirzinger, "A unified passivity-based control framework for position, torque and impedance control of flexible joint robots," The International Journal of Robotics Research, vol. 26, no. 1, pp. 23-39, 2007. [Online]. Available: https://doi.org/10.1177/0278364907073776

[156] S. Stramigioli, "Energy-aware robotics," in Mathematical Control Theory I, M. K. Camlibel, A. A. Julius, R. Pasumarthy, and J. M. Scherpen, Eds. Cham: Springer International Publishing, 2015, pp. 37-50.

[157] M. Franken, S. Stramigioli, S. Misra, C. Secchi, and A. Macchelli, "Bilateral telemanipulation with time delays: A two-layer approach combining passivity and transparency," IEEE Transactions on Robotics, vol. 27, no. 4, pp. 741-756, Aug 2011.

[158] A. Bicchi, S. L. Rizzini, and G. Tonietti, "Compliant design for intrinsic safety: general issues and preliminary design," in Proceedings 2001 IEEE/RSJ International Conference on Intelligent Robots and Systems. Expanding the Societal Role of Robotics in the the Next Millennium (Cat. No.01CH37180), vol. 4, 2001, pp. 1864-1869 vol.4.

[159] J. De Schutter, D. Torfs, H. Bruyninckx, and S. Dutré, "Invariant hybrid force/position control of a velocity controlled robot with compliant end effector using modal decoupling," vol. 16, pp. 340-356, 061997. 
[160] K. L. Doty, C. Melchiorri, and C. Bonivento, "A theory of generalized inverses applied to robotics," The International Journal of Robotics Research, vol. 12, no. 1, pp. 1-19, 1993. [Online]. Available: https: //doi.org/10.1177/027836499301200101

[161] M. Griffis and J. Duffy, "Kinestatic control: A novel theory for simultaneously regulating force and displacement," Journal of Mechanical Design, pp. 508515, 011991.

[162] S. Stramigioli and H. Bruyninckx, "Geometry and screw theory for robotics," in Proceedings IEEE International Conference on Robotics and Automation, Tutorial (T9), 2001.

[163] J. M. Selig, Geometrical Methods in Robotics, 1st ed. Secaucus, NJ, USA: Springer-Verlag New York, Inc., 1996.

[164] R. M. Murray, A mathematical introduction to robotic manipulation. CRC press, 2017.

[165] S. Stramigioli, Modeling and IPC Control of Interactive Mechanical Systems: A Coordinate-free Approach, (Lecture Notes). Springer-Verlag London Ltd, London, United Kingdom, 2001, p. 265.

[166] V. Duindam, "Port-based modeling and control for efficient bipedal walking robots," 32006.

[167] M. Zefran et al., "Choice of riemannian metrics for rigid body kinematics," vol. 2, 1996.

[168] R. W. Brockett, "Robotic manipulators and the product of exponentials formula," in Mathematical Theory of Networks and Systems, P. A. Fuhrmann, Ed. Berlin, Heidelberg: Springer Berlin Heidelberg, 1984, pp. 120-129.

[169] P. Grinfeld, Introduction to Tensor Analysis and the Calculus of Moving Surfaces. Springer, New York, NY, 2013.

[170] R. A. Sharipov, Quick introduction to tensor analysis: lecture notes. Online, 2013.

[171] M. Zefran and V. Kumar, "A geometrical approach to the study of the cartesian stiffness matrix," Journal of Mechanical Design, vol. 124, no. 1, pp. 30-38, 2002 .

[172] C. D. Meyer, Ed., Matrix analysis and applied linear algebra. Philadelphia, PA, USA: Society for Industrial and Applied Mathematics, 2000. [Online]. Available: http://matrixanalysis.com/DownloadChapters.html 
[173] G. Strang, "The fundamental theorem of linear algebra," The American Mathematical Monthly, vol. 100, no. 9, pp. 848-855, 1993. [Online]. Available: http://www.jstor.org/stable/2324660

[174] J. Watrous, The Theory of Quantum Information. Cambridge University Press, 2018.

[175] K. L. Doty, C. Melchiorri, E. M. Schwartz, and C. Bonivento, "Robot manipulability," IEEE Transactions on Robotics and Automation, vol. 11, no. 3, pp. 462-468, 1995.

[176] L. Qi, "Eigenvalues and invariants of tensors," Journal of Mathematical Analysis and Applications, vol. 325, no. 2, pp. 1363-1377, 2007.

[177] A. Ben-Israel and T. N. Greville, Generalized inverses: theory and applications. Springer Science \& Business Media, 2003, vol. 15.

[178] L. Merino, F. Caballero, J. R. Martínez-de Dios, I. Maza, and A. Ollero, “An unmanned aircraft system for automatic forest fire monitoring and measurement," Journal of Intelligent \& Robotic Systems, vol. 65, no. 1, pp. 533-548, Jan 2012.

[179] G. Kruijff et al., "Designing, developing, and deploying systems to support human-robot teams in disaster response," Advanced Robotics, vol. 28, no. 23, pp. 1547-1570, 2014.

[180] F. P. Kemper, K. A. O. Suzuki, and J. R. Morrison, "UAV consumable replenishment: design concepts for automated service stations," Journal of Intelligent \& Robotic Systems, vol. 61, no. 1, pp. 369-397, 2011.

[181] B. D. Song, J. Kim, J. Kim, H. Park, J. R. Morrison, and D. H. Shim, "Persistent UAV service: an improved scheduling formulation and prototypes of system components," in International Conference on Unmanned Aircraft Systems, 2013, pp. 915-925.

[182] D. R. Dale, "Automated ground maintenance and health management for autonomous unmanned aerial vehicles," Master's thesis, Department of Electrical Engineering and Computer Science, Massachusetts Institute of Technology, 2007.

[183] Y. Mulgaonkar, "Automated recharging for persistence missions with multiple micro aerial vehicles," Master's thesis, Department of Mechanical Engineering and Applied Mechanics, University of Pennsylvania, 2012.

[184] N. K. Ure, G. Chowdhary, T. Toksoz, J. P. How, M. A. Vavrina, and J. Vian, "An automated battery management system to enable persistent missions with multiple aerial vehicles," IEEE/ASME Transactions on Mechatronics, vol. 20, no. 1, pp. 275-286, 2015. 
[185] K. A. Swieringa, C. B. Hanson, J. R. Richardson, J. D. White, Z. Hasan, E. Qian, and A. Girard, "Autonomous battery swapping system for small-scale helicopters," in IEEE International Conference on Robotics and Automation, 2010, pp. 3335-3340.

[186] K. A. O. Suzuki, P. Kemper Filho, and J. R. Morrison, "Automatic battery replacement system for uavs: Analysis and design," Journal of Intelligent \& Robotic Systems, vol. 65, no. 1, pp. 563-586, 2012.

[187] K. Fujii, K. Higuchi, and J. Rekimoto, "Endless flyer: A continuous flying drone with automatic battery replacement," in 2013 IEEE 10th International Conference on Ubiquitous Intelligence and Computing and 2013 IEEE 10th International Conference on Autonomic and Trusted Computing, Dec 2013, pp. 216-223.

[188] R. Godzdanker, M. J. Rutherford, and K. P. Valavanis, "Islands: a self-leveling landing platform for autonomous miniature UAVs," in IEEE/ASME International Conference on Advanced Intelligent Mechatronics, 2011, pp. 170-175.

[189] F. Bourgault, T. Furukawa, and H. F. Durrant-Whyte, "Decentralized bayesian negotiation for cooperative search," in IEEE/RSJ International Conference on Intelligent Robots and Systems, 2004, pp. 2681-2686.

[190] J. Gancet, G. Hattenberger, R. Alami, and S. Lacroix, "Task planning and control for a multi-UAV system: architecture and algorithms," in IEEE/RSJ International Conference on Intelligent Robots and Systems, 2005, pp. 1017-1022.

[191] P. Doherty, J. Kvarnström, P. Rudol, M. Wzorek, G. Conte, C. Berger, T. Hinzmann, and T. Stastny, "A collaborative framework for 3D mapping using unmanned aerial vehicles," in Proceedings of the 19th International Conference on Principles and Practice of Multi-Agent Systems (PRIMA), Phuket, Thailand, 2016, pp. 110-130.

[192] J. Kim, B. D. Song, and J. R. Morrison, "On the scheduling of systems of UAVs and fuel service stations for long-term mission fulfillment," Journal of Intelligent \& Robotic Systems, vol. 70, no. 1, pp. 347-359, 2013. 



\section{ACKNOWLEDGEMENTS}

This thesis is the result of a lot of hard work and effort, but not only of mine alone. Many people contributed to it, and helped me out along the way.

First of all I want to thank my supervisors Stefano and Raffaella, for offering me the opportunity to pursue this degree, and for being patient with me and helping me to become a better researcher. A big thank you also goes to Stefan, who was a great help in the last push to finish this book, and to Matteo, for valuable guidance in the beginning of it.

The Robotics and Mechatronics group has become a home away from home, in no small part thanks to Jolanda, der guten Seele der Gruppe. My colleagues, especially Douwe and Geert, have stood by me with advice and valuable discussions. Marcel and Gerben, thank you for the help. Everyone in the group has contributed to a fantastic environment. This includes the many students, those of which I had the pleasure to work with, and those who sometimes kept me from working.

The SHERPA team not only put in hard work and long hours to conclude the project with me, but did this in a great spirit that I will miss. Especially Mark, without whom the project would not have been finished, and Jeroen. The international partners within SHERPA have made this demanding project a very rewarding experience, and managed to make the integration and review meetings fun.

Many friends came and went over the years, but certainly have left their mark. From fellow international and Dutch students, to my rugby team, I have met many great people in Enschede.

But most of all I need to thank my family, who have always supported me, and made it possible for me to be where I am now.

I have learned a lot at my time at the UT, and I hope I have grown somewhat as well. While it is time for new adventures now, I will look back at this chapter fondly.

Thank you to everyone who has made these last years special. 


\section{About the Author}

Éamon Barrett was born in Hamburg, Germany, in 1987. After completing his secondary education in 2006, he went on to study General Engineering Science, a wide-ranging BSc program teaching all branches of engineering, at the Hamburg University of Technology.

In 2010 he moved to the Netherlands to continue his studies with a MSc degree in Mechatronics at the University of Twente. Éamon worked on the miniaturization of variable stiffness actuators at the Robotics \& Mechatronics group during his individual assignment, and wrote his masters thesis on the design and control of a small quadrupedal robot based on these actuators.

After obtaining his MSc degree in 2013, he con-

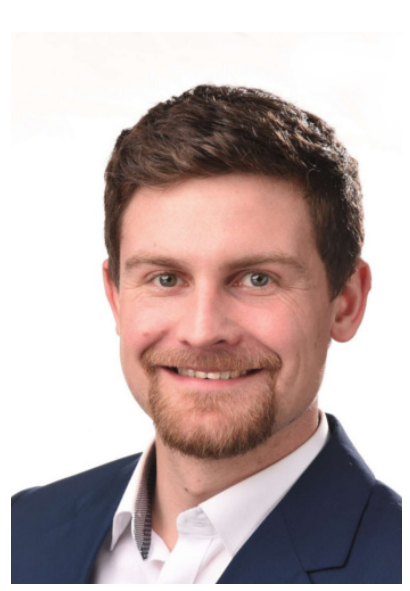
tinued working with the Robotics \& Mechatronics group in order to pursue a PhD degree on variable stiffness manipulation, as presented in this thesis. His $\mathrm{PhD}$ research was carried out as part of SHERPA, an ambitious but rewarding European project. The project's and group's engaged and talented members, interdisciplinary nature, and interesting applications are also the things that fascinate and inspire him about the field of robotics.

Éamon is continuing his research at the Italian Institute of Technology, where he joined the Advanced Robotics group. His research interests include mechanism design, compliant and variable stiffness actuation, and morphological computation. 


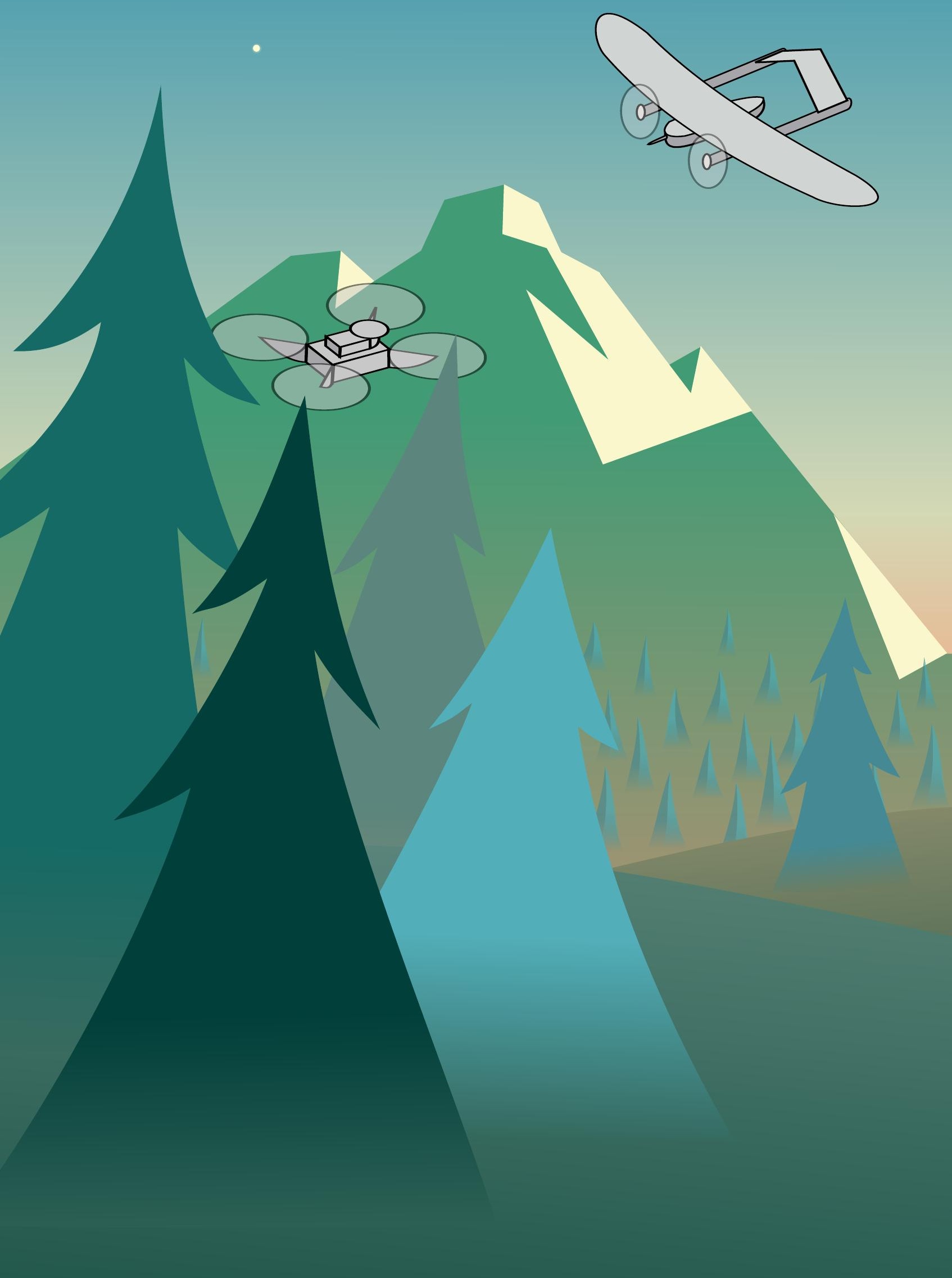

